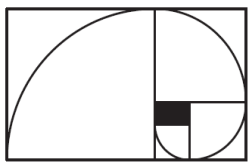

\title{
Pseudo-groupe d'une singularité de feuilletage holomorphe en dimension deux
}

\section{Frank Loray}

Résumé. Un feuilletage holomorphe singulier, en dimension deux, est localement défini par un champ de vecteur holomorphe à zéro isolé : les feuilles sont les trajectoires complexes du champ de vecteur. L'étude des singularités de ces feuilletages, débutée à la fin du 19ème siècle avec les travaux de Poincaré et Dulac, a connu un fort développement à partir des années 80 . Outre les problèmes de classification analytique, le thème qui nous intéresse particulièrement dans cet ouvrage est le lien entre l'existence d'un certain type d'intégrale première (multiforme) pour le feuilletage et la finitude de la dimension du pseudo-groupe d'holonomie. Par exemple, le feuilletage admet une intégrale première dans la classe de Liouville si, et seulement si, le pseudo-groupe est affine, de dimension deux.

La dimension est celle de la clôture du pseudo-groupe pour une topologie adéquate : nous comparerons dans cet ouvrage la clôture pour la convergence uniforme et la clôture de type Zariski introduite par Bernard Malgrange pour définir le groupoïde de Galois du feuilletage. Si la seconde conduit à une caractérisation simple et complète de l'intégrabilité du feuilletage, la première a l'avantage d'être de nature topologique/dynamique; les deux approches coïncident sur une large classe de feuilletages.

La première partie du texte est consacrée à l'étude des groupes de germes de difféomorphismes analytiques fixant $0 \in \mathbb{C}$, i.e. des sous-groupes de $\operatorname{Diff}(\mathbb{C}, 0)$. Après avoir rappelé les résultats de classifications formelle et analytique, nous donnons une description complète de la dynamique du pseudo-groupe induit sur un voisinage de 0 ainsi que ses clôtures pour les deux topologies précédentes. Comme résurgence du Théorème de Lie sur 
la classification des géométries de la droite, nous obtenons la dichotomie suivante : ou bien le pseudo-groupe est de dimension $\leq 2$ et sa dynamique est affine ou essentiellement discrète, ou bien il est de dimension infinie et sa clôture est le pseudo-groupe de toutes les transformations conformes. La seconde alternative est spécialement spectaculaire dans le cas de la topologie de convergence uniforme. Ces résultats reposent sur une étude complète des germes de difféomorphismes tangents à l'identité : ils apparaissent naturellement comme commutateurs des éléments de Diff $(\mathbb{C}, 0)$; c'est le cœur de la première partie de notre ouvrage.

Dans la seconde partie, nous rappelons la classification analytique des singularités réduites (ou non dégénérées) puis expliquons, à l'aide de la résolution des singularités par éclatements, comment décortiquer le pseudogroupe d'holonomie le long du diviseur exceptionnel. L'holonomie des composantes invariantes du diviseur en sont des ingrédients essentiels : ce sont des sous-groupes de Diff( $(\mathbb{C}, 0)$. Nous terminons par le résultat principal : un critère topologique d'intégrabilité pour une large classe de singularités. On y utilise toutes les notions développées durant ce livre ainsi que les résultats obtenus par Guy Casale sur la classification des groupoïdes de Galois et sur les intégrales premières d'un feuilletage.

Mots clefs. Feuilletage holomorphe, Pseudo-groupe, Singularité 
Abstract. A singular holomorphic foliation, in dimension two, is locally defined by a holomorphic vector field with isolated zero : leaves are trajectories of the vector field. The study of their singularities, which begun at the end of the 19th century through Poincare and Dulac works, had a huge development from the $80^{\prime}$. Beside analytic classification problems, we are mainly interested in this book by the links between the existence of (multiform) first integrals of a certain kind for the foliation and finitness of the dimension of the holonomy pseudo-group. For instance, the foliation admits a liouvillian first integral if, and only if, the pseudo-group is affine, of dimension two.

The dimension is that of the closure of the pseudo-group for a convenient topology : we will compare the closure of uniform convergence and the Zariski like closure introduced by Bernard Malgrange in order to define the Galois groupoid of the foliation. If the later approach leads to a simple and complete characterization of the integrability of the foliation, the former one has the advantage to be of topological/dynamical nature; the two definitions actually coincide for a large class of singularities.

The first part of the book is devoted to studying groups of germs of diffeomorphisms fixing $0 \in \mathbb{C}$, i.e. subgroups of $\operatorname{Diff}(\mathbb{C}, 0)$. After recalling formal and analytic classification results, we provide a complete descrition of the dynamics of the pseudo-group induced on the neighborhood of 0 as well as its closures for above topologies. A resurgence of Lie's classification of the geometries of the line arise in the following dichotomy : either the pseudo-group has dimension at most two and is affine or discrete, or it is infinite dimensional and its closure is the whole conformal pseudogroup. Those results relie on a complete study of tangent to the identity diffeomorphisms : they naturally appear as commutators of elements of $\operatorname{Diff}(\mathbb{C}, 0)$; they play a fundamental role in the first part of the book.

In the second part, we recall the analytic classification of reduced (or non degenerate) singularities of foliations and then explain how to recover the holonomy pseudo-group of a general singularity from the resolution by blowing-ups. The holonomy of the invariant components of the exceptional divisor after blowing-up the main generators of the pseudo-group : they are subgroups of $\operatorname{Diff}(\mathbb{C}, 0)$. We end-up by the main result : a topological criterium of integrability for a huge class of foliation singularities. Here, we use most of the tools developped along this book as well as results obtained by Guy Casale on the classification of Galois groupoids and first integrals for foliations.

Keywords. Holomorphic Foliation, Pseudo-group, Singularity

2020 Mathematics Subject Classification: Primary: 32S65. Secondary: 22Exx, $34 \mathrm{Cxx}, 34 \mathrm{Mxx}, 37 \mathrm{~F} 75,57 \mathrm{Sxx}, 58 \mathrm{Hxx}$. 


\section{Table des matières}

I Sous-groupes de $\operatorname{Diff}(\mathbb{C}, 0) \quad 67$

2 Classification formelle et propriétés algébriques $\quad 68$

2.1 Sous-algèbres de Lie de $\mathcal{X}(\mathbb{C}, 0) \ldots \ldots . \ldots . \ldots . \ldots 68$

2.2 Sous-groupes à 1 paramètre de $\operatorname{Diff}(\mathbb{C}, 0) \ldots \ldots . \ldots . \ldots 73$

2.3 Classification formelle des éléments de $\operatorname{Diff}(\mathbb{C}, 0) \ldots 76$

2.4 Normalisation formelle des sous-groupes résoluble de Diff( $\mathbb{C}, 0) \quad$. $\quad 80$

2.5 Sous-groupes non résolubles de $\operatorname{Diff}(\mathbb{C}, 0):$ quelques invariants . . 83

2.6 Quelques problèmes ouverts . . . . . . . . . . . . . 88

3 Classification analytique $\quad \mathbf{9 3}$

3.1 Linéarisation et problèmes de petits diviseurs . . . . . . . . . . . 93

3.2 Les difféomorphismes tangents à l'identité : survol historique . . 100

3.3 Le théorème de la fleur . . . . . . . . . . . . . . . . . . . . . . . . . . . . . . . 105

3.4 Trivialisations sectorielles . . . . . . . . . . . . . . 107

3.5 La cohomologie des trivialisation sectorielles . . . . . . . . . . . . 112

3.6 Le chapelet de sphères . . . . . . . . . . . . . . . . . . . . . 115

3.7 Les invariants holomorphes . . . . . . . . . . . . . . . . 117

3.8 Les symétries du chapelet . . . . . . . . . . . . . . . . . . . . 122

3.9 Classification topologique . . . . . . . . . . . . . . 128

3.10 Classification analytique des sous-groupes résolubles de $\operatorname{Diff}(\mathbb{C}, 0) \quad 129$

3.11 Remarques sur les sous-groupes non résolubles de $\operatorname{Diff}(\mathbb{C}, 0)$. . . 133

4 Dynamique des sous-groupes de type fini de $\operatorname{Diff}(\mathbb{C}, 0) \quad 134$

4.1 Dynamiques résolubles . . . . . . . . . . . . . . . . 135

4.2 Dynamiques non résolubles . . . . . . . . . . . . . . . . . . . . 144

4.3 Le Théorème de de Nakai . . . . . . . . . . . . . . . . . . . . 147

4.4 Clôture topologique d'un pseudo-groupe et pseudo-algèbre de Lie 155

4.5 Clôture de Malgrange . . . . . . . . . . . . . . . . . . . 162 


\section{Singularités de feuilletages holomorphes en di- mension 2

5 Feuilletages holomorphes et pseudo-groupes $\quad \mathbf{1 7 4}$

5.1 Feuilletages réguliers sur les surfaces complexes . . . . . . . . . . 174

5.2 Pseudo-groupes d'un feuilletage . . . . . . . . . . . . . . . . 176

5.3 Holonomie d'une feuille, d'un feuilletage . . . . . . . . . . . . . . 180

5.4 Feuilletages singuliers . . . . . . . . . . . . . . . . . 181

6 Singularités réduites $\quad \mathbf{1 8 5}$

6.1 Bestaire préliminaire des singularités non dégénérées . . . . . . . 185

6.2 Singularités réduites linéaires . . . . . . . . . . . . . . . . . . . . . . . . . . . 188

6.3 Singularités réduites de type col . . . . . . . . . . . . . . . . . 189

6.4 Singularités réduites de type noud-col . . . . . . . . . . . . . . . 195

7 Réduction des singularités $\quad 202$

7.1 Éclatements . . . . . . . . . . . . . . . . . . . . . . 202

7.2 La formule de l'indice . . . . . . . . . . . . . . . . . . . . . . 212

7.3 Pseudo-groupes d'holonomie projective . . . . . . . . . . . 216

7.4 Construction de singularités non réduites . . . . . . . . . . . . 219

8 Structure transverse d'une singularité générale $\mathbf{2 2 6}$

8.1 Correspondances de Dulac . . . . . . . . . . . . . . . . 226

8.2 Les générateurs du pseudo-groupe d'holonomie . . . . . . . . . 233

8.3 Holonomie et intégrales premières . . . . . . . . . . . . . . . . . . 237

8.4 Passage de coins . . . . . . . . . . . . . . . . . . 249

$\begin{array}{lr}\text { Bibliography } & \mathbf{2 5 7}\end{array}$ 


\section{Chapitre 1}

\section{Introduction}

La notion de feuilletage apparaît implicitement à la fin du 19ème siècle dans les travaux de Henri Poincaré et Paul Painlevé, dans le but d'étudier de manière qualitative les solutions des équations différentielles analytiques

$$
F\left(x, y, y^{\prime}, \ldots, y^{(n)}\right)=0
$$

où $F$ est polynomiale (éventuellement analytique dans la variable $x$ ). Par exemple, l'équation différentielle $y^{\prime}=\frac{f(x, y)}{g(x, y)}$ définit un feuilletage holomorphe singulier là où $f$ et $g$ sont définies, holomorphes : les feuilles sont les trajectoires complexes du champ de vecteur $X=f(x, y) \partial_{x}+g(x, y) \partial_{y}$, les singularités sont les points d'indétermination du second membre, i.e. les zéros de $X$. On peut supposer $f$ et $g$ sans facteur commun de sorte que les singularités sont isolées dans le plan. L'étude du feuilletage au voisinage d'un point singulier s'est tout d'abord développée dans les travaux de $\mathrm{H}$. Poincaré (théorème de linéarisation), puis de H. Dulac, notamment motivés par le 16 ème problème de Hilbert sur les cycles limites des champs de vecteurs réels polynomiaux du plan. Ensuite, il a fallu attendre plus d'un demi siècle avant que ces feuilletages singuliers ne soient reconsidérés. Entre temps, la théorie des feuilletages réguliers sur les variétés compactes s'est développée de manière tout à fait indépendante, motivée par la topologie de ces variétés, à partir des années 50 avec les travaux de G. Reeb, C. Ehresmann et A. Haeffliger. L'étude des feuilletages analytiques singuliers n'est réapparue que dans le courant des années 70, en France avec les travaux de R. Moussu, J.-F. Mattei, D. Cerveau, J. Martinet et J.-P. Ramis, en Russie avec V.I. Arnol'd, Yu. Ilyashenko, A.A. Scherbakov, S. Voronin et P. Elizarov et au Brésil avec I. Kupka, C. Camacho, A. Lins Neto et P. Sad. À partir des années 80 , des résultats profonds ont été démontrés notamment sur les problèmes de classification analytique.

En 1980, suite à des questions de R. Thom, J.-F. Mattei et R. Moussu démontrent dans l'article fondateur [140] qu'un germe de feuilletage sin- 
gulier admet une intégrale première holomorphe si, et seulement si, toutes les feuilles sont fermées sur un voisinage épointé du point singulier et si un nombre fini d'entre elles seulement adhèrent au point singulier. Ceci nous donne une caractérisation topologique de l'existence d'intégrale première holomorphe. Depuis, de nombreux travaux ont cherché à caractériser les feuilletages admettant des intégrales premières d'un type plus général telles que les fonctions multiformes de Darboux, de Liouville, etc... Citons notamment [57], [115], [218], [161], [199], [15], [220] et enfin [48] qui semble clore le sujet. Comme il est remarqué dans [148], le critère de MatteiMoussu revient à dire que le quotient Hausdorff d'un voisinage épointé de la singularité par la relation induite par le feuilletage est un disque : c'est l'espace des feuilles. En général, l'espace des feuilles d'un feuilletage est un objet à la fois très riche et très compliqué dont le plus petit quotient Hausdorff est bien souvent réduit à un point. On préfère alors travailler avec le pseudo-groupe d'holonomie : c'est la donnée d'une transversale complète $T$ au feuilletage (i.e. intersectant toutes les feuilles) et de la collection $G$ des "applications de retour" partielles induites par le feuilletage sur $T$; le quotient de $T$ par l'action de $G$ est l'espace des feuilles. C'est un pseudo-groupe totalement discontinu (essentiellement dénombrable modulo prolongement analytique) très difficile à étudier en toute généralité.

Le cas des pseudo-groupes continus et transitifs est complètement élucidé par S. Lie en dimension 1 : un tel pseudo-groupe est localement conjugué au pseudo-groupe des transformations euclidiennes (translations), affines ou projectives de la droite dès lors qu'il est de dimension finie. Si le pseudogroupe du feuilletage considéré plus haut se plonge dans un des pseudogroupes de Lie de dimension finie précédent, disons le plus gros, alors le feuilletage est transversalement projectif et la développante de la structure transverse nous fournit une intégrale première multiforme à monodromie projective. Dans le cas singulier, de telles intégrales premières deviennent uniformes et méromorphes dès qu'elles sont définies sur le complémentaire de la singularité, par simple connexité; on doit accepter des structures transverses dégénérant le long d'un ensemble de codimension 1 si l'on veut voir apparaître des exemples non triviaux. Par exemple, si le feuilletage est défini par une 1-forme méromorphe fermée $\omega$, alors le pseudo-groupe d'holonomie préserve cette 1-forme en restriction à la transversale et, en l'intégrant localement, on déduit que le feuilletage est transversalement euclidien en dehors des pôles de $\omega$. Réciproquement, si le feuilletage est transversalement euclidien en dehors d'une courbe et si la développante $H$ est à croissance polynômiale le long de cette courbe, alors le feuilletage est défini par la 1-forme fermée $d H$, qui est méromorphe par Riemann. Ceci nous donne une définition naturelle de feuilletage transversalement euclidien dans le cas singulier. Dans [199], B. Scardua propose les définitions analogues suivantes, adaptées au contexte holomorphe singulier : le feuilletage défini par une 1-forme holomorphe $\omega_{0}$ est transversalement projectif 
s'il existe des 1-formes méromorphes $\omega_{1}$ et $\omega_{2}$ telles que

$$
\left\{\begin{array}{l}
d \omega_{0}=\omega_{0} \wedge \omega_{1}, \\
d \omega_{1}=\omega_{0} \wedge \omega_{2}, \\
d \omega_{2}=\omega_{1} \wedge \omega_{2},
\end{array}\right.
$$

le feuilletage est transversalement affine lorsque l'on peut choisir $\omega_{2}=0$. Dans le cas des feuilletages réguliers, se donner une structure affine ou projective est en effet équivalent à se donner de telles 1-formes régulières (voir [85]). En 1992, M.F. Singer démontre dans [209] qu'un germe de feuilletage holomorphe singulier admet une intégrale première dans la classe de Liouville si, et seulement si, le feuilletage est transversalement affine au sens précédent; la développante, donnée par $H=\int e^{-\int \omega_{1}} \omega_{0}$, est dans la classe de Liouville. C'est ce résultat qui a motivé la définition précédente et tous les développements qui ont suivi.

Étant donnée une singularité de feuilletage générale, si l'on veut se ramener à la discussion précédente, on a essentiellement deux possibilités : ou bien on arrive à plonger directement le pseudo-groupe $G$ du feuilletage dans les pseudo-groupes de Lie de la droite, ou bien on cherche d'abord à définir la clôture de $G$ pour une topologie adéquate, puis son algèbre de Lie à laquelle on applique le Théorème de Lie. Les deux approches seront abordées ici ; la seconde donnera notamment des critères de non existence d'intégrale première, lorsque la clôture sera de dimension infinie.

Deux topologies seront considérées pour définir la clôture d'un pseudogroupe, et son algèbre de Lie. La première, de nature dynamique, est donnée par la convergence uniforme sur les compacts. Nous détaillerons la construction de l'adhérence $\bar{G}$ de $G$ pour cette topologie ainsi que son algèbre de Lie (réelle!). Cette approche, déjà implicite dans le travail de Nakai [151], a été développée dans [12], [124] et [120]. Dans [127], B. Malgrange propose une autre topologie de nature plus analytique : l'adhérence $\bar{G}^{\text {Lie }}$ de $G$ apparaît comme limite inductive des adhérences de Zariski des relevés de $G$ dans les espaces de jets. Dans le cas de la dimension 1, les pseudo-groupes fermés pour cette topologie ont été complètement classifiés par Guy Casale dans [47]. L'avantage de cette seconde approche est de donner un critère simple et complet d'existence d'intégrale première particulière : dans [48], Guy Casale démontre qu'un feuilletage singulier est transversalement projectif au sens précédent si, et seulement si, $\bar{G}^{\text {Lie }}$ est de dimension 3 ; en particulier, le feuilletage admet une intégrale première dans la classe de Liouville si, et seulement si, $\bar{G}^{\text {Lie }}$ est de dimension 2 . Il est facile de voir que cette topologie est plus grossière que la précédente, c'està-dire $G \subset \bar{G} \subset \bar{G}^{\text {Lie }}$. Par contre, l'avantage de l'approche dynamique est qu'elle est stable par homéomorphisme : si deux feuilletages sont conjugués par un homéomorphisme, alors celui-ci conjugue les clôtures respectives $\bar{G}$ ainsi que leurs algèbres de Lie et, par suite, les dimensions coïncident. 
En ce sens, on est ici plus proche de l'esprit du critère de Mattei-Moussu pour les intégrales premières holomorphes. Par exemple, lorsque $\bar{G}$ est de dimension infinie, nous obtenons un critère topologique de non existence d'intégrale première à la Khovanskii (voir [97]).

Une partie consistante du pseudo-groupe d'une singularité de feuilletage est donnée, après résolution de la singularité par éclatements, par l'holonomie des composantes irréductibles du diviseur exceptionnel : les applications de retour, lorsque l'on contourne les nouvelles singularités apparaissant le long du diviseur, nous fournissent une représentation du groupe fondamental dans le groupe des germes de difféomorphismes d'une transversale donnée, fixant le diviseur. À travers une coordonnée locale, on récupère un sous-groupe de

$$
\operatorname{Diff}(\mathbb{C}, 0)=\{f(z)=a z+\cdots ; f \in \mathbb{C}\{z\}, a \neq 0\} .
$$

L'étude de la dynamique d'un élément de $\operatorname{Diff}(\mathbb{C}, 0)$ remonte à la naissance des systèmes dynamiques avec les travaux de E. Schröder, G. Køenigs et L. Leau à la fin du 19ème siècle. Le sujet s'est considérablement développé suite aux travaux de G. Julia et P. Fatou sur l'itération des polynômes quadratiques. Par contre, l'étude de la dynamique d'un sous-groupe n'est apparue que bien plus tard, motivée par celle des feuilletages, dans un cadre global ou local, avec les articles [91], [50] et [58]. Dans ce dernier papier, un début de classification des sous-groupes résolubles apparaît; l'énoncé final, qui est démontré indépendamment dans [115, 123] et [74], dit qu'un sous-groupe résoluble de $\operatorname{Diff}(\mathbb{C}, 0)$ induit une dynamique affine sur un voisinage épointé dès lors que sont premier groupe dérivé est de rang $\geq 2$. Les sous-groupe non résolubles ont été étudiés par A.A. Shcherbakov dans [203], [205] et [206, 207] puis par I. Nakai dans [151] : en général, les orbites sont denses sur un voisinage de 0 . Rien ne permet alors de distinguer une telle dynamique de celle d'un sous-groupe résoluble générique; c'est dans [12] qu'apparaît la dichotomie profonde : la clôture d'un sous-groupe non résoluble de $\operatorname{Diff}(\mathbb{C}, 0)$ pour la convergence uniforme est le pseudo-groupe de toutes les transformations conformes. Un sous-groupe non résoluble est en ce sens de dimension infinie, même du point de vue topologique/dynamique.

Le but de la première partie est de démontrer la dichotomie dynamique précédente entre sous-groupes résolubles et non résolubles de $\operatorname{Diff}(\mathbb{C}, 0)$. La démonstration de ces résultats repose en grande partie sur une bonne compréhension des difféomorphismes tangents à l'identité. Ils apparaissent naturellement comme commutateurs des éléments de Diff( $\mathbb{C}, 0)$. Nous leurs consacrons une bonne partie du chapitre 3. Nous y démontrons notamment le théorème de classification analytique dû indépendamment à J. Écalle et S.M. Voronin, en adoptant plutôt le point de vue de J. Martinet et J.-P. Ramis sur l'espace des orbites : le chapelet de sphères. Un autre ingrédient important est le Théorème de Nakai sur les dynamiques non résolubles, 
que nous démontrons dans le chapitre 4 : en particulier, on y démontre que l'algèbre de Lie associée à la clôture topologique du pseudo-groupe est non triviale. C'est l'étape la plus difficile. Ensuite, on se ramène assez facilement au théorème de Lie pour conclure à la dimension infinie. À la fin de cette première partie, nous abordons sans entrer dans les détails la clôture du pseudo-groupe au sens de Malgrange ; ceci est très bien fait dans le travail de Guy Casale [47].

Dans la seconde partie, nous expliquons comment utiliser les notions développées dans la première partie pour étudier le pseudo-groupe d'une singularité de feuilletage générale. Il y a moins de démonstrations, certaines étant renvoyées aux articles originaux. Nous rappelons la classification analytique des singularités réduites (ou non dégénérées) puis expliquons, à l'aide de la résolution des singularités par éclatements, comment décortiquer le pseudo-groupe d'holonomie le long du diviseur exceptionnel. L'holonomie des composantes invariantes du diviseur en sont des ingrédients essentiels : ce sont des sous-groupes de $\operatorname{Diff}(\mathbb{C}, 0)$. L'autre ingrédient que nous détaillons dans la dernière section est l'étude des correspondances de Dulac qui permettent de reconnecter les pseudo-groupes d'holonomie le long du diviseur. Nous décrivons rapidement le dictionnaire établi par Guy Casale dans [48] entre intégrales premières et dimension du groupoïde de Galois.

Nous terminons ce livre par une application de toutes les notions introduites durant ce cours : le Théorème 8.30. On considère ici une singularité de feuilletage $\mathcal{F}$ dont le diviseur exceptionnel $D$, après résolution par éclatements, est totalement invariant par le feuilletage relevé $\widetilde{\mathcal{F}}$. On suppose en outre que toutes les singularités (réduites) de $\widetilde{\mathcal{F}}$ sont de type col ou hyperbolique, i.e. localement définies par $x d y+\alpha y d x+\cdots=0$ avec $\alpha \in \mathbb{C} \backslash \mathbb{R}^{-}$(pas de nœud ni de nœud-col). Alors tout germe de transversale $T$ au diviseur exceptionnel (en un point régulier de $\widetilde{\mathcal{F}}$ ) est une transversale complète : elle coupe toutes les feuilles sauf un nombre fini contenu dans la courbe invariante de $\mathcal{F}$ (quitte à diminuer le domaine de définition de $\mathcal{F})$. Le pseudo-groupe $G$ du feuilletage est alors donné par sa restriction à $T$ et est de l'un des types suivants :

- $G$ est discret, $G=\bar{G}$, et est induit par un sous-groupe virtuellement abélien de $\operatorname{Diff}(\mathbb{C}, 0)$ sur $T$,

- $\bar{G}$ est de dimension finie $>0(G$ non discret $)$ et $\mathcal{F}$ est transversalement projectif,

- $\bar{G}$ est de dimension infinie et contient toutes les transformations conformes en un point générique de $T$.

Les sous-groupes de $\operatorname{Diff}(\mathbb{C}, 0)$ apparaissant dans le premier cas sont complètement classifiés dans la première partie de cet ouvrage. On ne peut pas toujours décider de l'intégrabilité du feuilletage correspondant par un critère topologique : les singularités réduites de type col résonant par exemple nous fournissent des feuilletages topologiquement conjugués dont le grou- 
poïde de Galois peut être de dimension arbitraire $0,1,2,3$ ou $\infty$.

Dans le second cas, le pseudo-groupe $\bar{G}$ se redresse en un point générique de $T$ sur (le pseudo-groupe induit par) un sous-groupe de Lie réel de $\operatorname{PGL}(2, \mathbb{C})$; sa clôture analytique $\bar{G}^{\text {Lie }}$ correspond alors à la clôture de Zariski complexe du groupe de Lie réel associé. La définition de $\bar{G}$ étant de nature topologique, tout feuilletage $\mathcal{F}^{\prime}$ topologiquement conjugué à $\mathcal{F}$ aura même type d'intégrale première : leur groupoïde de Galois ont même dimension transverse 1,2 ou 3 .

Dans le troisième cas, nous obtenons un critère topologique de non intégrabilité.

La preuve du Théorème 8.30 consiste en grande partie à reprendre la stratégie développée par Emmanuel Paul dans [161] en l'adaptant à notre point de vue topologique : en reconstruisant le pseudo-groupe $G$ de proche en proche le long du diviseur $D$, on étudie comment peuvent évoluer les dimensions des clôtures $\bar{G}$ et $\bar{G}^{\text {Lie }}$. À chaque fois que $\bar{G}^{\text {Lie }}$ est de dimension infinie, on observe a posteriori (sous nos hypothèses) ou bien que $G$ est induit par un sous-groupe de $\operatorname{Diff}(\mathbb{C}, 0)$, ou bien que $\bar{G}$ est aussi de dimension infinie. Dans le second cas, les techniques de [12] permettent de conclure que $\bar{G}$ est le pseudo-groupe conforme sur un ouvert de Zariski réel de $T$. $A$ contrario, lorsque $\bar{G}^{\text {Lie }}$ est de dimension finie, les résultats de $[47,48]$ nous permettent de conclure à l'existence d'une géométrie transverse au feuilletage, et par suite à l'existence d'intégrale première de type spécial.

Le Théorème 8.30 reste vrai en remplaçant le diviseur de résolution d'une singularité par tout diviseur connexe invariant par un feuilletage singulier dont les singularités sont de type col ou hyperbolique. Par exemple, si $D$ est le diviseur obtenu après résolution d'un feuilletage $\mathcal{F}$ de $\mathbb{P}^{2}(\mathbb{C})$ le long d'une courbe invariante, alors on obtient une version topologique du genre d'énoncés que l'on trouve dans $[32,33]$ : Dans ce cas, toute intégrale première est en fait globale et de nature algébrique : par exemple, les 1-formes méromorphes $\omega_{0}, \omega_{1}, \omega_{2}$ définissant la structure projective sont rationnelles.

\section{Mode d'emploi}

Le lecteur intéressé par les difféomorphismes à une variable pourra lire les sections 1.1 à 1.4 pour la classification formelle des difféomorphismes, groupes à 1 paramètre et sous-groupes résolubles. La classification analytique est décrite dans les sections 2.1, puis 2.3 à 2.7, et enfin 2.10. Enfin les aspects dynamiques sont abordés dans les sections 3.1 et 3.2. Pour l'étude d'un seul difféomorphisme (et non pas d'un groupe), il suffira de regarder les sections 1.3, 2.1, 2.3 à 2.7, et enfin 3.1.

Les autres sections (1 à 3) de la première partie abordent des questions plus pointues. Par exemple, la section 3.3 donne une preuve du théorème de 
Nakai sur les dynamiques non résolubles (et utilise les résultats techniques de la section 1.5). La section 1.6 aborde le problème de la structure de groupe (relations, alternative de Tits). La section 2.2 est un survol un peu historique sur l'étude des difféomorphismes tangents à l'identité. La section 2.8 explique comment on peut utiliser le chapelet de sphère pour bien comprendre divers types de symétries du difféomorphisme. On trouve dans la section 2.9 le théorème de Camacho sur la classification topologique et dans la section 2.11 la rigidité de Cerveau-Moussu entre classification formelle et analytique pour les sous-groupes non virtuellement cycliques. Enfin, 3.4 et 3.5 abordent les problèmes de complétude de la dynamique du point de vue de la convergence uniforme dans 3.4 et d'adhérence de Zariski dans 3.5 (groupoïde de Malgrange et résultats de Casale).

Concernant les feuilletages, les sections 4 à 6 sont une introduction aux notions de feuilletages holomorphes singuliers (section 4), classification des singularités réduites (section 5), désingularisation (section 6.1), de séparatrice (section 6.2), d'holonomie projective (section 6.3) et de construction de feuilletages à holonomie prescrite (section 6.4).

La section 7 aborde des questions plus pointues, notamment les liens entre holonomie et intégrales premières dans laa section 7.3 , partant des travaux de Mattéi-Moussu jusqu'aux travaux de É. Paul et G. Casale. Enfin la section 7.4 donne des caractérisations topologiques de l'existence de certaines intégrales premières en utilisant tous les ingrédients de cet ouvrage; les résultats obtenus sont originaux et peuvent être vus comme une version topologique du travail de É. Paul sur l'intégration liouvillienne (dont on utilise d'ailleurs les techniques).

\section{Remerciements}

Je tiens tout d'abord à remercier chaleureusement D. Cerveau, M. Berthier, F. Cano, M. Nicolau, P. Sad, É. Ghys et D. Gaboriau pour m'avoir maintes fois encouragé à écrire ce livre. Sans leur insistance infatigable, il n'aurait certainement jamais vu le jour. Je remercie aussi mes co-auteurs R. Meziani, I. Liousse, M. Belliart et J. Rebelo qui m'ont aidé à comprendre et à faire avancer l'étude des sous-groupes de Diff( $\mathbb{C}, 0)$. Enfin, je remercie aussi X. Buff, C. Camacho, G. Casale, J. Écalle, P. Elizarov, A. Lins Neto, J.-M. Lion, J.-F. Mattei, R. Moussu, I. Nakai, E. Paul, J.P. Ramis, J. Ribon, É. Salem, B. Scardua et F. Touzet qui m'ont aidé toutes ces années, à travers de nombreuses discussions, à connaitre le sujet dans ses moindres recoins et à simplifier de nombreuses démonstrations. Et merci surtout à Guy Casale qui, par sa relecture minutieuse, a relevé de nombreuses coquilles. 
L'origine de ce texte vient d'un cours que Felipe Cano m'avait invité à donner à Tordesillas l'hiver 1997, en parallèle avec des cours d'Étienne Ghys et de Jean-Marie Lion. Une première version portait alors le titre de 5 leçons sur la structure transverse d'une singularité de feuilletage holomorphe en dimension 2 complexe. J'ai repris ce texte à l'automne 2005 où j'ai pris le temps de compléter avec les résultats obtenus entre temps par Bernard Malgrange et Guy Casale sur la définition de groupoïde et le lien avec les intégrales premières. Ce texte est depuis devenu mon texte le plus cité, bien que n'ayant toujours pas vraiment d'existence officielle. Ensaios Matematicos m'a récemment contacté pour me proposer de le soumettre pour publication et je tiens à remercier son éditeur Mario Jorge Dias Carneiro. L'idée de publier dans une revue brésilienne me plait beaucoup puisque ces recherches résultent en grande partie de mes collaborations et discussions avec les collègues brésiliens. Je pense que ce texte reste le plus complet sur les sous-groupes de $\operatorname{Diff}(\mathbb{C}, 0)$ et j'espère que sa publication sera utile pour tous ceux désirant apprendre plus dans ce sujet.

L'auteur est employé par le CNRS, et il remercie le projet ANR-16CE40-0008 "Foliage", ainsi que le projet CAPES-COFECUB MA 932/19. 



\section{Première partie}

\section{Sous-groupes de $\operatorname{Diff}(\mathbb{C}, 0)$}




\section{Chapitre 2}

\section{Classification formelle et propriétés algébriques}

Notons $\widehat{\operatorname{Diff}}(\mathbb{C}, 0)$ le groupe pour la composition des "germes de difféomorphismes formels" fixant $0 \in \mathbb{C}$ :

$$
\widehat{\operatorname{Diff}}(\mathbb{C}, 0)=\{f(z)=a z+\cdots ; f \in \mathbb{C}[[z]], a \neq 0\} .
$$

Nous décrivons les propriétés algébriques de $\operatorname{Diff}(\mathbb{C}, 0)$, ou plus généralement de $\widehat{\operatorname{Diff}}(\mathbb{C}, 0)$. Nous donnons notamment une classification des éléments et sous-groupes résolubles de $\operatorname{Diff}(\mathbb{C}, 0)$ à conjugaison près dans $\widehat{\operatorname{Diff}}(\mathbb{C}, 0)$ (classification formelle). Nous terminerons par une description assez sommaire des sous-groupes non résolubles et aborderons quelques questions ouvertes dans ce domaine.

\subsection{Sous-algèbres de Lie de $\mathcal{X}(\mathbb{C}, 0)$}

On note $\mathcal{X}(\mathbb{C}, 0)$ l'algèbre de Lie des germes de champs de vecteurs holomorphes en $0 \in \mathbb{C}$ :

$$
\mathcal{X}(\mathbb{C}, 0)=\left\{X=f(z) \cdot \partial_{z} ; f \in \mathbb{C}\{z\}\right\} ;
$$

le crochet de Lie de deux éléments est donné par

$$
\left[f \partial_{z}, g \partial_{z}\right]=\left(f \cdot g^{\prime}-f^{\prime} \cdot g\right) \partial_{z} .
$$

Une sous-algèbre $\mathcal{L}$ de $\mathcal{X}(\mathbb{C}, 0)$ est un sous-espace vectoriel complexe stable par crochet de Lie. On dit que $\mathcal{L}$ est transitive si l'un au moins de ses éléments ne s'annule pas en 0 ; on dira qu'elle est intransitive sinon.

Commençons par rappeler le résultat classique de Sophus Lie sur la classification des géométries de la droite (voir [109]) 
Théorème 2.1 (Lie). Soit $\mathcal{L} \subset \mathcal{X}(\mathbb{C}, 0)$ une sous-algèbre de Lie transitive de dimension finie. Alors $\mathcal{L}$ est de dimension $\leq 3$ et, après changement de coordonnées, s'identifie à l'une des algèbres suivantes :

1. $\mathbb{C}\left\langle\partial_{z}\right\rangle$,

2. $\mathbb{C}\left\langle\partial_{z}, z \partial_{z}\right\rangle$,

3. $\mathbb{C}\left\langle\partial_{z}, z \partial_{z}, z^{2} \partial_{z}\right\rangle$.

Ceci signifie, par exemple dans le cas 1 , qu'il existe $\varphi \in \operatorname{Diff}(\mathbb{C}, 0)$ tel que l'algèbre $\mathcal{L}$ est engendrée par $\varphi^{*} \partial_{z}=\frac{1}{\varphi^{\prime}(z)} \partial_{z}$. Les groupes de Lie correspondant aux modèles précédents sont les groupes de transformations des géométries euclidienne, affine et projective de la droite :

1. $\mathbb{E}:=\{f(z)=z+t ; t \in \mathbb{C}\}$,

2. $\mathbb{A}:=\left\{f(z)=a z+b ; a \in \mathbb{C}^{*}, b \in \mathbb{C}\right\}$,

3. $\mathbb{P}:=\left\{f(z)=\frac{a z+b}{c z+d} ; a, b, c, d \in \mathbb{C}, a d-b c \neq 0\right\}$.

Le théorème de Lie sera démontré dans quelques instants en même temps que sa version intransitive. Avant cela, disons un mot des sous-algèbres de dimension infinie.

Notons $J^{k}$ l'application qui à une série formelle associe son jet d'ordre $k$ :

$$
J^{k}: \mathbb{C}[[z]] \rightarrow \mathbb{C}_{k}[z] ; \sum_{n \geq 0} a_{n} z^{n} \mapsto \sum_{n=0}^{k} a_{n} z^{n} .
$$

Proposition 2.2. Soit $\mathcal{L} \subset \mathcal{X}(\mathbb{C}, 0)$ une sous-algèbre de Lie transitive de dimension infinie. Alors $J^{k} \mathcal{L}=\mathbb{C}_{k}[z] \partial_{z}$ pour tout $k \in \mathbb{N}$.

On prendra garde que $\mathcal{L} \neq \mathcal{X}(\mathbb{C}, 0)$ en général (par exemple, $\mathcal{L}=$ $\left.\mathbb{C}[z] \partial_{z}\right)$. Toutefois, il résulte de la proposition qu'il n'existe pas de structure géométrique homogène complexe autre que les trois géométries citées plus haut.

Les sous-algèbres intransitives de $\mathcal{X}(\mathbb{C}, 0)$ sont celles qui vont donner naissance aux sous-groupes de Lie de $\operatorname{Diff}(\mathbb{C}, 0)$. D'abord, commençons par rappeler la classification analytique des éléments de $\mathcal{X}(\mathbb{C}, 0)$ (voir [216])

Proposition 2.3 (Szekeres). Soit $X \in \mathcal{X}(\mathbb{C}, 0)$ un germe de champ de vecteurs holomorphe non trivial. Alors, à changement de coordonnée près, on est dans l'un des cas suivants :

$-X=\partial_{z}$;

$-X=\alpha z \partial_{z}, \alpha \in \mathbb{C}^{*}$

$-X=\frac{z^{p+1}}{1+\frac{\lambda}{2 i \pi} z^{p}} \partial_{z}, p \in \mathbb{N}^{*}, \lambda \in \mathbb{C}$.

Démonstration. Notons $X=f(z) \partial_{z}$ et considérons la 1-forme différentielle méromorphe duale $\omega=\frac{d z}{f(z)}$. Celle-ci est caractérisée par le fait que $\omega(X) \equiv$ 
1 : elle est intrinsèquement définie. Il est donc équivalent de classifier les 1-formes méromorphes du type $\omega=\frac{d z}{f(z)}$ à changement de coordonnée près.

Lorsque $f(0) \neq 0$, la 1 -forme $\omega$ est holomorphe, régulière et se redresse sur la forme constante $d z$ par changement de coordonnée $\varphi(z):=\int_{0}^{z} \frac{d \zeta}{f(\zeta)}$. Ainsi, $X=\varphi^{*} \partial_{z}$.

Lorsque $f$ s'annule à l'origine, $\omega$ a un pôle. Son résidu $\lambda$ est invariant par changements de coordonnée. Lorsque ce pôle est simple, on cherche à conjuguer $\omega$ à $\lambda \frac{d z}{z}$. Pour cela, on écrit $\omega=\lambda \frac{d z}{z}+d g$, avec $g(z)$ holomorphe, puis on égalise

$$
\omega=\varphi^{*} \lambda \frac{d z}{z}=\lambda \frac{d \varphi}{\varphi} .
$$

En posant $\varphi=z u(z)$, il vient $d g=\alpha \frac{d u}{u}$ et $u:=e^{g / \lambda}$ convient $: u(0) \neq 0$ et $\varphi \in \operatorname{Diff}(\mathbb{C}, 0)$ redresse $X$ sur $\alpha z \partial_{z}$, où $\alpha=1 / \lambda$.

Enfin, lorsque $\omega$ possède un pôle multiple, son ordre $p+1$ est aussi un invariant. On cherche alors à conjuguer $\omega$ à $\frac{d z}{z^{p+1}}+\frac{\lambda}{2 i \pi} \frac{d z}{z}$. Comme précédemment, on écrit $\omega=\frac{\lambda}{2 i \pi} \frac{d z}{z}+d\left(\frac{g}{z^{p}}\right)$, avec $g(z)$ holomorphe, puis on égalise

$$
\omega=\frac{d \varphi}{\varphi^{p+1}}+\frac{\lambda}{2 i \pi} \frac{d \varphi}{\varphi} .
$$

En posant de nouveau $\varphi=z u(z)$, il vient

$$
-\frac{1}{p u^{p}}+\frac{\lambda}{2 i \pi} z^{p} \log (u)=g
$$

et une solution holomorphe non nulle $u(z)$ nous est donnée par le théorème des fonctions implicites. Ainsi, $\varphi$ redresse $X$ sur le champ de vecteurs $\frac{z^{p+1}}{1+\frac{\lambda}{2 i \pi} z^{p}} \partial_{z}$.

Remarque 2.4. Dans la suite, on notera $X_{p, \lambda}$ la forme normale obtenue dans le cas dégénéré

$$
X_{p, \lambda}:=\frac{z^{p+1}}{1+\frac{\lambda}{2 i \pi} z^{p}} \partial_{z} .
$$

Notons que $\left(z^{p+1}-\frac{\lambda}{2 i \pi} z^{2 p+1}\right) \partial_{z}$ est conjugué à $X_{p, \lambda}$ (voir la démonstration précédente) et aurait tout aussi bien pu convenir comme forme normale. Cependant, $X_{p, \lambda}$ a l'avantage que sa forme duale $\omega_{p, \lambda}=\frac{d z}{z^{p+1}}+\frac{\lambda}{2 i \pi} \frac{d z}{z}$ admet une primitive bien plus simple, à savoir :

$$
\psi_{p, \lambda}=-\frac{1}{p z^{p}}+\frac{\lambda}{2 i \pi} \log (z) .
$$

Cette transformation (multiforme) redresse $X_{p, \lambda}$ sur le champ constant $\partial_{z}$ et sera maintes fois utilisée plus loin. 
Remarque 2.5. Le champ de vecteurs $t X_{p, \lambda}, t \in \mathbb{C}$, est l'image par l'homothétie $\varphi(z)=\tau z, \tau^{p}=t$, du champ $X_{p, \frac{\lambda}{t}}$. En particulier, l'algèbre de Lie $\mathbb{C}\left\langle X_{p, \lambda}\right\rangle$ est conjuguée à $\mathbb{C}\left\langle X_{p, 1}\right\rangle$ dès que $\lambda \neq 0$.

Théorème 2.6 (Version intransitive). Soit $\mathcal{L} \subset \mathcal{X}(\mathbb{C}, 0)$ une sous-algèbre de Lie non triviale intransitive de dimension finie. Alors $\mathcal{L}$ est de dimension $\leq 2$ et, à changement de coordonnée près, on est dans l'un des cas suivants :

$-\mathbb{C}\left\langle z \partial_{z}\right\rangle$,

$-\mathbb{C}\left\langle z^{p+1} \partial_{z}\right\rangle, p \in \mathbb{N}^{*}$

$-\mathbb{C}\left\langle\left(z^{p+1}+z^{2 p+1}\right) \partial_{z}\right\rangle, p \in \mathbb{N}^{*}$,

$-\mathbb{C}\left\langle z \partial_{z}, z^{p+1} \partial_{z}\right\rangle, p \in \mathbb{N}^{*}$.

Démonstration des Théorèmes 2.1 et 2.6 et de la Proposition 2.2. $\mathrm{Si} \mathcal{L}$ contient 4 champs de vecteurs indépendants (resp. 3 dans le cas intransitif), alors, par combinaisons linéaires, on peut supposer que $\mathcal{L}$ en contient 2 du type :

$$
X=\left(z^{p+1}+\cdots\right) \partial_{z} \quad \text { et } \quad Y=\left(z^{q+1}+\cdots\right) \partial_{z}
$$

avec $0<p<q$. Par crochets itérés :

$$
\begin{aligned}
{[X, Y] } & =\left((q-p) z^{p+q+1}+\cdots\right) \partial_{z} \\
{[X,[X, Y]] } & =\left(q(q-p) z^{2 p+q+1}+\cdots\right) \partial_{z}, \\
{[X,[X,[X, Y]]] } & =\left((q+p) q(q-p) z^{3 p+q+1}+\cdots\right) \partial_{z}, \text { etc } \ldots
\end{aligned}
$$

on déduit une suite d'éléments de l'algèbre dérivée $\{\mathcal{L}, \mathcal{L}\}$ de plus en plus dégénérés. Dans ce cas, $\{\mathcal{L}, \mathcal{L}\}$ est de dimension infinie et, en réitérant cet argument, on déduit de plus que $\mathcal{L}$ n'est pas résoluble.

Supposons maintenant $\mathcal{L}$ de dimension finie. Dans le cas intransitif, $\mathcal{L}$ est de dimension 1 ou 2. Dans le premier cas, nous sommes ramenés à la Proposition 2.3 et nous utilisons les Remarques 2.4 et 2.5 pour nous ramener aux 3 premiers modèles de l'énoncé du Théorème 2.6. Dans le second cas, $\mathcal{L}$ est engendrée par deux éléments de la forme

$$
X=(z+\cdots) \partial_{z} \quad \text { et } \quad Y=\left(z^{p+1}+\cdots\right) \partial_{z}
$$

avec $p \in \mathbb{N}^{*}$. On choisit une coordonnée dans laquelle $X=z \partial_{z}$ (voir Proposition 2.3) et la condition de stabilité par crochet nous dit que $Y=$ $z^{p+1} \partial_{z}$.

Dans le cas transitif, $\mathcal{L}$ est de dimension $\leq 3$ et engendrée par un élément transitif, disons $X=\partial_{z}+\cdots$, et par la sous-algèbre $\mathcal{L}_{0}$ des éléments qui fixent 0 . En redressant $\mathcal{L}_{0}$ sur un des modèles donnés soit par la Proposition 2.3, soit par la discussion précédente, on vérifie que la stabilité

$$
\left[X, \mathcal{L}_{0}\right] \subset \mathbb{C}\left\langle X, \mathcal{L}_{0}\right\rangle
$$


n'est satisfaite que lorsque $\mathcal{L}_{0}=\mathbb{C}\left\langle z \partial_{z}\right\rangle$ ou $\mathbb{C}\left\langle z \partial_{z}, z^{2} \partial_{z}\right\rangle$; alors, la condition

$$
\left[X, z \partial_{z}\right] \subset \mathbb{C}\left\langle X, \mathcal{L}_{0}\right\rangle
$$

entraine dans chacun des cas que $X=\partial_{z}$ modulo $\mathcal{L}_{0}$.

Finalement, supposons $\mathcal{L}$ transitive et de dimension infinie : elle contient un élément de la forme $X=\partial_{z}+\cdots$ et des éléments $Y=\left(z^{p+1}+\cdots\right) \partial_{z} \in \mathcal{L}$ d'ordre arbitrairement grand. Par crochets itérés $[X, \ldots[X,[X, Y]] \cdots]$ on construit des éléments $X_{q}=\left(z^{q+1}+\cdots\right) \partial_{z} \in \mathcal{L}$ de tout ordre $q+1$ pour $q=0, \ldots, p$.

Remarque 2.7. Tous les énoncés précédents restent vrai lorsque l'on remplace $\mathcal{X}(\mathbb{C}, 0)$ par l'algèbre de Lie des germes de champs de vecteurs formels en $0 \in \mathbb{C}$ :

$$
\widehat{\mathcal{X}}(\mathbb{C}, 0)=\left\{X=f(z) \cdot \partial_{z} ; f \in \mathbb{C}[[z]]\right\} ;
$$

les formes normales s'obtiennent alors par conjugaison formelle $\widehat{\varphi} \in$ $\widehat{\operatorname{Diff}}(\mathbb{C}, 0)$.

Remarque 2.8. On peut être plus précis que dans la Proposition 2.2 dès lors que l'on suppose l'algèbre de Lie $\mathcal{L}$ fermée dans $\mathcal{X}(\mathbb{C}, 0)$ ou $\widehat{\mathcal{X}}(\mathbb{C}, 0)$ pour une topologie raisonnable. Par exemple, on peut équiper $\widehat{\mathcal{X}}(\mathbb{C}, 0)$ de la topologie de Krull

$$
f_{n} \rightarrow f \quad \Leftrightarrow \quad \forall k \in \mathbb{N}, J^{k} f_{n}=J^{k} f \text { pour } n>>0
$$

ou mieux par la topologie plus fine

$$
f_{n} \rightarrow f \quad \Leftrightarrow \quad \forall k \in \mathbb{N},\left\|J^{k}\left(f_{n}-f\right)\right\|_{k} \rightarrow 0
$$

où $\|\cdot\|_{k}$ est n'importe quelle norme sur l'espace $\mathbb{C}_{k}[z]$ des polynômes de degré $\leq k$. Par ailleurs, on peut aussi munir $\mathbb{C}\{z\}$ (et par suite $\mathcal{X}(\mathbb{C}, 0)$ ) de la topologie analytique

$$
f_{n} \rightarrow f \quad \Leftrightarrow \quad \exists r>0,\left\|f_{n}-f\right\|_{r} \rightarrow 0
$$

ò̀ $\|f\|_{r}=\sup _{\{|z|<r\}}|f(z)|$ avec la convention $\|f\|_{r}=\infty$ lorsque $f$ ne converge pas sur le disque de rayon $r$ (voir [141]). On déduit immédiatement de la Proposition 2.2 que ni $\mathcal{X}(\mathbb{C}, 0)$, ni $\widehat{\mathcal{X}}(\mathbb{C}, 0)$ ne contiennent de sous-algèbre fermée transitive stricte de dimension infinie pour l'une ou l'autre de ces topologies.

Exercice 2.9. Donner la liste des sous-algèbres de Lie réelles $\mathcal{L}_{\mathbb{R}} \subset$ $\mathcal{X}(\mathbb{C}, 0)$ modulo $\operatorname{Diff}(\mathbb{C}, 0)$. On remarquera que $\mathcal{L}_{\mathbb{R}}$ est contenue dans une sous-algèbre complexe $\mathcal{L}_{\mathbb{R}} \subset \mathcal{L}_{\mathbb{C}} \subset \mathcal{X}(\mathbb{C}, 0)$ de dimension moindre ou égale. 


\subsection{Sous-groupes à 1 paramètre de $\operatorname{Diff}(\mathbb{C}, 0)$}

Tout élément $X \in \mathcal{X}(\mathbb{C}, 0)$ définit un flot holomorphe $\phi_{X}^{t}$ au voisinage de 0 .

Proposition 2.10. Étant donné $X \in \mathcal{X}(\mathbb{C}, 0)$, l'équation différentielle

$$
\frac{d}{d t} \phi(t, z)=f(\phi(t, z)), \quad X=f(z) \partial_{z}
$$

admet une unique solution holomorphe $\phi=\phi_{X}^{t}(z) \in \mathbb{C}\{t, z\}$ satisfaisant $\phi_{X}^{0}(z)=z$. Elle satisfait en outre la loi de groupe à 1 paramètre

$$
\phi_{X}^{0}(z)=z \quad \text { et } \quad \phi_{X}^{t} \circ \phi_{X}^{s}(z)=\phi_{X}^{t+s}(z)
$$

pour $t, s, z \in \mathbb{C}$ suffisamment proches de 0 .

Réciproquement, si $\phi^{t}(z) \in \mathbb{C}\{t, z\}$ satisfait $(2.3)$ pour $t, s, z \in \mathbb{C}$ proches de 0 , alors $\phi^{t}(z)=\phi_{X}^{t}(z)$ où $X=f(z) \partial_{z}$ est le champ de vecteurs défini par

$$
f=\left[\frac{\partial}{\partial t} \phi(t, z)\right]_{t=0} .
$$

On dit que $X$ est le générateur infinitésimal du flot $\phi_{X}^{t}$.

Démonstration. Considérons dans $\left(\mathbb{C}^{2}, 0\right)$ le germe de champ de vecteurs holomorphe $\partial_{t}+f(z) \partial_{z}$. Puisque ce champ est régulier et transverse à la droite verticale $\{t=0\}$, le théorème de redressement affirme qu'il existe un unique changement de coordonnées holomorphe de la forme $\Phi(t, z)=$ $(t, \phi(t, z))$ satisfaisant

$$
\Phi_{*} \partial_{t}=\partial_{t}+f(z) \partial_{z} \quad \text { et } \quad \Phi(0, z)=(0, z) \text {. }
$$

Par construction, $\phi$ est la solution recherchée. La loi de groupe se déduit alors de l'invariance du champ de vecteurs $\partial_{t}+f(z) \partial_{z}$ par translations $(t, z) \mapsto(t+c, z), c \in \mathbb{C}$.

Réciproquement, si $\phi^{t}(z) \in \mathbb{C}\{t, z\}$ satisfait (2.3) pour $t, s, z \in \mathbb{C}$ proches de 0 , alors

$$
\frac{\partial}{\partial t} \phi^{t}(z)=\lim _{s \rightarrow 0} \frac{\phi^{t+s}(z)-\phi^{t}(z)}{s}=\lim _{s \rightarrow 0} \frac{\phi^{s}\left(\phi^{t}(z)\right)-\phi^{0}\left(\phi^{t}(z)\right)}{s}=f\left(\phi^{t}(z)\right) .
$$

Remarque 2.11. On peut aussi définir le flot de $X \in \mathcal{X}(\mathbb{C}, 0)$ par convergence uniforme, pour $t, z \in \mathbb{C}$ proches de 0 , de

$$
\phi_{X}^{t}(z):=\lim _{n \rightarrow \infty}\left(z+\frac{t}{n} f(z)\right)^{\circ n}
$$


où $\varphi^{\text {on }}$ désigne l'itérée $n^{\text {ème }}$ de $\varphi$. En effet, si f est définie et uniformément bornée par $c>0$ sur le disque $\mathbb{D}_{r}=\{|z|<r\}$ pour un rayon $r>0$, alors $|f(z)| \leq c|z|$ et l'itérée $n^{\text {ème }}$ de $z+\frac{t}{n} f(z)$ est bornée sur le disque de rayon $r e^{-t c}$ par:

$$
\left|\left(z+\frac{t}{n} f(z)\right)^{\circ n}\right| \leq c e^{-t c}\left(1+\frac{t c}{n}\right)^{n} \leq c
$$

(ceci montre en même temps que les itérées sont bien définies sur $\mathbb{D}_{c e^{-t}}$ ). Par contre, il est assez délicat de vérifier proprement que toute suite extraite convergente (donnée par le théorème de Montel) satisfait la loi de groupe à 1 paramètre (2.3).

En général, le domaine de définition de $\phi_{X}^{t}$ décroit au fûr et à mesure que $t$ devient grand, mais il contient toujours un voisinage de 0 dès lors que $X$ est singulier, i.e. $f(0)=0$. Dans ce cas, on hérite d'un homomorphisme de groupes

$$
\mathbb{C} \rightarrow \operatorname{Diff}(\mathbb{C}, 0) ; t \mapsto \phi_{X}^{t}
$$

Notons $\mathcal{X}^{0}(\mathbb{C}, 0)$ la sous-algèbre de codimension 1 de $\mathcal{X}(\mathbb{C}, 0)$ formée des germes de champs de vecteurs s'annulant à l'origine. Un élément $X \in$ $\mathcal{X}^{0}(\mathbb{C}, 0)$ agit par dérivation sur $\mathbb{C}\{z\}$; on notera $f \mapsto X \cdot f$ cette dérivation et $f \mapsto X^{n} \cdot f$ ses itérées : $X^{k+1} \cdot f=X \cdot\left(X^{k} \cdot f\right)$.

Proposition 2.12. Étant donné $X \in \mathcal{X}^{0}(\mathbb{C}, 0)$ et $f \in \mathbb{C}\{z\}$, l'expression

$$
\exp (t X) \cdot f=\sum_{n \geq 0} \frac{t^{n}}{n !} X^{n} \cdot f
$$

converge pour tout $t \in \mathbb{C}$ définissant un opérateur différentiel (d'ordre infini) sur $\mathbb{C}\{z\}$ qui satisfait

$$
\exp (t X) \cdot f=f \circ \phi_{X}^{t} \quad \text { pour tout } \quad f \in \mathbb{C}\{z\} .
$$

En particulier, on a $\phi_{X}^{t}(z)=\sum_{n \geq 0} \frac{t^{n}}{n !} X^{n} \cdot z$.

Démonstration. Par analyticité de $X$ et de $f$, on peut développer

$$
f \circ \phi_{X}^{t}(z)=\sum_{n \geq 0} \frac{t^{n}}{n !}\left[\frac{\partial^{n}}{\partial t^{n}} f \circ \phi_{X}^{t}\right]_{t=0} .
$$

Par ailleurs, l'équation différentielle satisfaite par $\phi_{X}^{t}$ (Proposition 2.10) nous donne

$$
\frac{\partial}{\partial t}\left(f \circ \phi_{X}^{t}\right)=(X \cdot f) \circ \phi_{X}^{t}
$$

et plus généralement

$$
\frac{\partial^{n}}{\partial t^{n}}\left(f \circ \phi_{X}^{t}\right)=\left(X^{n} \cdot f\right) \circ \phi_{X}^{t}
$$


En évaluant en $t=0$, on obtient les coefficients

$$
\left[\frac{\partial^{n}}{\partial t^{n}} f \circ \phi_{X}^{t}\right]_{t=0}=X^{n} \cdot f
$$

Les Propositions 2.10 et 2.12 s'adaptent au cas formel : tout élément $X \in \widehat{\mathcal{X}}^{0}(\mathbb{C}, 0)$ définit un unique homomorphisme

$$
\mathbb{C} \rightarrow \widehat{\operatorname{Diff}}(\mathbb{C}, 0) ; t \mapsto \phi_{X}^{t}
$$

défini ou bien comme solution formelle de l'équation $\frac{d}{d t} \phi_{X}^{t}=f \circ \phi_{X}^{t}$, ou bien par $\phi_{X}^{t}(z)=\sum_{n \geq 0} \frac{t^{n}}{n !} X^{n} \cdot z$. En fait, l'opérateur différentiel $X$ et ses itérées commutent avec la projection sur les jets

$$
J^{k}: \mathbb{C}[[z]] \rightarrow \mathbb{C}_{k}[z] \partial_{z} ; \sum_{n \geq 0} a_{n} z^{n} \mapsto \sum_{n=0}^{k} a_{n} z^{n}
$$

et on a $J^{k} \phi_{X}^{t}=J^{k} \phi_{J^{k} X}^{t}$ ce qui montre la convergence de l'exponentielle comme série formelle $\phi_{X}^{t}(z) \in \mathcal{O}\left(\mathbb{C}_{t}\right)[[z]]$ dont les coefficients sont des séries entières en $t$.

Considérons maintenant un sous-groupe à 1 paramètre

$$
(\mathbb{C},+) \rightarrow \operatorname{Diff}(\mathbb{C}, 0) ; t \mapsto \phi^{t} .
$$

On dira qu'il est holomorphe lorsqu'il est défini au voisinage de $t=z=0$ par un flot holomorphe $\phi^{t}(z) \in \mathbb{C}\{t, z\}$. Une conséquence immédiate des Propositions 2.3 et 2.10 est le

Corollaire 2.13. Soit $(\mathbb{C},+) \rightarrow \operatorname{Diff}(\mathbb{C}, 0) ; t \mapsto \phi^{t}$ un sous-groupe à 1 paramètre holomorphe. Alors il existe $\varphi \in \operatorname{Diff}(\mathbb{C}, 0)$ et $\lambda \in \mathbb{C}^{*}$ tels que $\varphi^{*} \phi^{\lambda t}$ soit l'un des groupes à 1 paramètre suivants

$-t \mapsto \phi^{t}(z)=\exp \left(t z \partial_{z}\right) \cdot z=e^{t} z$,

$-t \mapsto \phi^{t}(z)=\exp \left(t z^{p+1} \partial_{z}\right) \cdot z=z /\left(1+t z^{p}\right)^{1 / p}, p \in \mathbb{N}^{*}$,

$-t \mapsto \phi^{t}(z)=\exp \left(t\left(z^{p+1}+z^{2 p+1}\right) \partial_{z}\right) \cdot z, p \in \mathbb{N}^{*}$.

Ici, la racine $p^{\text {ème }}\left(1+t z^{p}\right)^{1 / p}$ est choisie de sorte que $(1)^{1 / p}=1$; cette écriture n'a bien sûr de sens que pour $t z^{p}$ proche de 0 .

Démonstration. D'après la Proposition 2.10, $\phi^{t}=\phi_{X}^{t}$ pour un germe de champ de vecteurs holomorphe $X$ (fixant 0 ). D'après la Proposition 2.3, $\varphi^{*} X$ est ou bien de la forme $\lambda z \partial_{z}$, ou bien de la forme $\left(z^{p+1}+\frac{\lambda}{2 i \pi} z^{2 p+1}\right) \partial_{z}$ avec $\lambda \in \mathbb{C}$ et $p \in \mathbb{N}^{*}$. Après reparamétrisation, on arrive aux modèles ci-dessus en tenant compte du fait que

$$
\phi_{\varphi^{*} X}^{t}=\varphi^{*} \phi_{X}^{t} .
$$


Remarque 2.14. Le flot de $X_{p, \lambda}=t\left(z^{p+1}+\frac{\lambda}{2 i \pi} z^{2 p+1}\right) \partial_{z}$ n'est explicite que pour $\lambda=0$. Des résultats de I.N. Baker et J. Écalle montrent qu'aucun élément du flot (excepté $\phi_{X}^{0}$ ) ne peut être algébrique, ni même conjugué dans $\operatorname{Diff}(\mathbb{C}, 0)$ à une branche de fonction algébrique. On peut toutefois calculer les premiers coefficients avec (2.6) :

$$
\phi_{X_{p, \lambda}}^{t}(z)=\exp \left(t . X_{p, \lambda}\right) \cdot z=z+t z^{p+1}+\left(\frac{p+1}{2} t^{2}-\frac{\lambda}{2 i \pi} t\right) z^{2 p+1}+\cdots
$$

Remarque 2.15. On peut construire des sous-groupes à 1 paramètre artificiels en composant par exemple n'importe quel modèle ci-dessus par un homomorphisme de groupes $(\mathbb{C},+) \rightarrow(\mathbb{C},+)$. C'est pour cette raison qu'un minimum de régularité sur $t$ est nécessaire. On obtiendra un énoncé plus fort en classifiant tous les sous-groupes abéliens de Diff( $\mathbb{C}, 0)$ plus loin. Notons tout de même que si les coefficients d'un homomorphisme

$$
\mathbb{R} \rightarrow \operatorname{Diff}(\mathbb{C}, 0) ; t \mapsto \phi^{t}(z)=\sum_{n>0} a_{n}(t) z^{n}
$$

dépendent continûment de $t$, alors ils en dépendent analytiquement; en effet, $J^{k} \phi^{t}$ définit alors un homomorphisme continu $(\mathbb{C},+) \rightarrow J^{k} \operatorname{Diff}(\mathbb{C}, 0)$ dans un groupe de Lie de dimension finie, et est par suite analytique (réel).

\subsection{Classification formelle des éléments de $\operatorname{Diff}(\mathbb{C}, 0)$}

Nous dirons que deux éléments $f, g \in \operatorname{Diff}(\mathbb{C}, 0)$ ou $\widehat{\operatorname{Diff}}(\mathbb{C}, 0)$ sont formellement conjugués s'il existe $\hat{\varphi} \in \widehat{\operatorname{Diff}}(\mathbb{C}, 0)$ tel que $g=\hat{\varphi}_{*} f=$ $\hat{\varphi} \circ f \circ \hat{\varphi}^{\circ(-1)}$. Un premier invariant de conjugaison formelle est donné par la partie linéaire $a=f^{\prime}(0)=g^{\prime}(0)$. La Proposition qui suit est un analogue discret de la Proposition 2.3.

Proposition 2.16. Soit $f=a z+\cdots \in \widehat{\operatorname{Diff}}(\mathbb{C}, 0), a \in \mathbb{C}^{*}$. Alors $f$ est formellement conjugué à :

- $f_{0}(z)=a z$ lorsque $a \in \mathbb{C}^{*}$ n'est pas périodique,

- $f_{0}(z)=$ az lorsque a est d'ordre $q \in \mathbb{N}^{*}$ et $f^{\circ q}=$ identité,

- $f_{0}=a \exp \left(X_{k q, \lambda}\right), k \in \mathbb{N}^{*}$ lorsque a est d'ordre $q \in \mathbb{N}^{*}$ mais $f^{\circ q} \neq$ identité.

Démonstration. On élimine de proche en proche les coefficients non linéaires de $f$ de la façon suivante. Si $f(z)=a z+b z^{n+1}+\cdots$, alors on pose $\varphi_{n}(z)=z+c z^{n+1}$ et :

$$
\left(\varphi_{n}\right)_{*} f=\varphi_{n} \circ f \circ\left(\varphi_{n}\right)^{\circ(-1)}(z)=a z+\left(b-a\left(1-a^{n}\right) c\right) z^{n+1}+\cdots
$$


de sorte que si $a^{n} \neq 1$, en choisissant $c$ convenablement, on élimine le coefficient d'ordre $n+1$ et on avance. Si $a$ n'est pas périodique ou encore si, à chaque fois que $a^{n}=1$, le coefficient $b$ correspondant est déjà nul, l'algorithme permet de construire une coordonnée formelle $\hat{\varphi}:=\cdots \circ \varphi_{n} \circ$ $\cdots \circ \varphi_{2} \circ \varphi_{1}$ telle que $\hat{\varphi}_{*} f(z)=a z$.

Sinon, c'est-à-dire si, à un certain moment :

$$
f(z)=a z+b z^{k q+1}+\cdots \quad \text { avec } a \text { d'ordre } q, k \in \mathbb{N}^{*} \text { et } b \in \mathbb{C}^{*},
$$

alors, par un changement de coordonnée linéaire, on normalise $b=a$ puis par un nouvel algorithme empilant des changements de coordonnée du type $\varphi_{n}$, on construit une coordonnée formelle $\hat{\varphi}$ ramenant $f$ à la forme polynomiale :

$$
f(z)=a z+a z^{k q+1}+a c z^{2 k q+1}, \quad c \in \mathbb{C} .
$$

Plus précisément, lorsque $f(z)=a z+a z^{k q+1}+b z^{n+1}+\cdots$ avec $k q<$ $n<2 k q$ ou, après, lorsque $f(z)=a z+a z^{k q+1}+a c z^{2 k q+1}+b z^{n+1}+\cdots$ avec $2 k q<n$, on élimine le coefficient $b$ par un changement de coordonnée du type $\varphi_{n}$ si $a^{n} \neq 1$ et du type $\varphi_{n-k q}$ si $a^{n}=1$. Maintenant, le même algorithme appliqué à :

$$
a \exp \left(X_{p, \lambda}\right)(z)=a z+a z^{p+1}+\left(\frac{p+1}{2}-\frac{\lambda}{2 i \pi}\right) a z^{2 p+1}+\cdots
$$

permet d'identifier formellement $f$ à $a \exp \left(X_{k q, \lambda}\right)$ avec $c=\frac{p+1}{2}-\frac{\lambda}{2 i \pi}$.

Étant donné $f(z)=a z+\sum_{n>2} a_{n} z^{n} \in \widehat{\operatorname{Diff}}(\mathbb{C}, 0)$ avec $a$ périodique, il résulte de la démonstration précédente qu'une infinité de conditions sont nécessaires sur les coefficients $a_{n}$ de $f$ pour assurer la périodicité (et donc la linéarisabilité) de $f$. On dit que $f$ est résonant lorsqu'il n'est pas linéarisable.

L'élément $\hat{\varphi} \in \widehat{\operatorname{Diff}}(\mathbb{C}, 0)$ conjugant $f$ à son modèle, $\hat{\varphi}_{*} f=f_{0}$, est unique à composition près (à gauche) par un élément du centralisateur formel de $f_{0}$ :

$$
\widehat{\operatorname{Cent}}\left(f_{0}\right)=\left\{g \in \widehat{\operatorname{Diff}}(\mathbb{C}, 0) ; f_{0} \circ g=g \circ f_{0}\right\} .
$$

Autrement dit :

$$
\hat{\varphi}_{*} f=\hat{\psi}_{*} f=f_{0} \quad \Leftrightarrow \quad \hat{\psi}=g \circ \hat{\varphi} \text { avec } g \in \widehat{\operatorname{Cent}}\left(f_{0}\right) .
$$

Ceci ne laisse en général que peu de possibilités pour $g$ comme le montre la :

Proposition 2.17. L'ensemble des éléments $g \in \widehat{\operatorname{Diff}}(\mathbb{C}, 0)$ qui commutent aux modèles formels $f_{0}$ énumérés dans la Proposition 2.16 sont respectivement donnés par : 


$$
\begin{aligned}
& -\widehat{\operatorname{Cent}}\left(f_{0}\right)=\left\{g(z)=b z ; b \in \mathbb{C}^{*}\right\} \\
& -\widehat{\operatorname{Cent}}\left(f_{0}\right)=\left\{g(z)=z \cdot \hat{g}\left(z^{q}\right) ; g \in \mathbb{C}[[z]]\right\}, \\
& -\widehat{\operatorname{Cent}}\left(f_{0}\right)=\left\{g(z)=e^{2 i \pi p / k q} \cdot \exp \left(t . X_{k q, \lambda}\right) \cdot z ; p \in \mathbb{Z} / k q \mathbb{Z} \text { et } t \in \mathbb{C}\right\} .
\end{aligned}
$$

Notons que le centralisateur est convergent (dans $\operatorname{Diff}(\mathbb{C}, 0)$ ) et abélien excepté dans le cas où $f_{0}$ est périodique.

Démonstration. Dans les deux premiers cas, on les calcule directement : on identifie $g(a z)=a g(z)$ en substituant une série formelle à $g$. Dans le troisième cas, si $g$ commute au modèle formel, il commute aussi à son itérée $q^{\text {ème }}$ qui est de la forme $f^{\circ q}(z)=z+q z^{k q+1}+\cdots$; un calcul immédiat montre que $g(z)=e^{2 i \pi p / k q} z+\cdots$. On écrit $g=e^{2 i \pi p / k q} g_{1}$ avec $g_{1}$ tangent à l'identité. Visiblement, $e^{2 i \pi p / k q} z$ commute à $X_{k q, \lambda}$ et donc à son flot; par suite, $g_{1}$ commute à $f^{\circ q}$ et un calcul immédiat montre que $g_{1}(z)=$ $z+t z^{k q+1}+\cdots$. De nouveau, on écrit $g_{1}=\exp \left(t X_{k q, \lambda}\right) \circ g_{n}$ avec $g_{n}(z)=$ $z+c z^{n+1}+\cdots$ tangent à l'identité à l'ordre $n>k q$; ce dernier doit lui aussi commuter à $f^{\circ q}$ et on vérifie aisément que ça ne peut être que l'identité.

Considérons maintenant l'application exponentielle (ou flot au temps 1)

$$
\exp : \widehat{\mathcal{X}}^{0}(\mathbb{C}, 0) \rightarrow \widehat{\operatorname{Diff}}(\mathbb{C}, 0) ; X \mapsto \exp (X) \cdot z
$$

définie par (2.6). Comme nous l'avons déjà remarqué, elle commute avec la projection sur les jets d'ordre $k$ dans le sens suivant :

$$
J^{k} \exp (X)=J^{k} \exp \left(J^{k} X\right)
$$

et en particulier, nous notons que

$$
\begin{array}{rlrl}
\exp \left(\alpha z \partial_{z}+\cdots\right) \cdot z & =e^{\alpha} z+\cdots & \alpha \in \mathbb{C}^{*} \\
\exp \left(c z^{p+1} \partial_{z}+\cdots\right) \cdot z & = & z+c z^{p+1}+\cdots & c \in \mathbb{C} .
\end{array}
$$

Une conséquence des Propositions 2.3 et 2.16 est que l'application exponentielle n'est ni injective, ni surjective. Plus précisément :

Corollaire 2.18. Soit $f=a z+\cdots \in \widehat{\operatorname{Diff}}(\mathbb{C}, 0), a \in \mathbb{C}^{*}$. Alors $f=\exp (\hat{X})$ pour un champ de vecteur formel $\hat{X}=\alpha z \partial_{z}+\cdots \in \widehat{\mathcal{X}}(\mathbb{C}, 0)$ si, et seulement si, on est dans l'un des cas suivants :

- a n'est pas périodique et dans ce cas, $\hat{X}=\alpha z \partial_{z}+\cdots$ est unique dès que l'on a choisit $\alpha$ tel que $a=e^{\alpha}$;

- a et $f$ sont périodiques et dans ce cas, l'ensemble des champs de vecteurs $\hat{X}$ tels que $f=\exp (\hat{X})$ est de dimension infinie;

- $a=1$ et dans ce cas $\hat{X}$ est unique.

En particulier, un difféomorphisme résonant $f=a \exp \left(X_{k q, \lambda}\right)$ n'est le flot au temps 1 d'un champ de vecteur formel que si $a=1$. 
Démonstration. Dans chacun des cas, on considère les modèles formels donnés par la Proposition 2.16. Lorsque $f_{0}(z)=a z$, bien entendu $X_{0}=$ $\alpha z \partial_{z}$ convient pour n'importe quel $\alpha$ tel que $a=e^{\alpha}$. Soit $\hat{X}=\alpha z \partial_{z}+\cdots$ un autre champ de vecteurs formel tel que $\exp (\hat{X})=f_{0}$. Supposons d'abord $\alpha \neq 0$. Alors, d'après la Proposition 2.3 (version formelle), il existe $\hat{\varphi} \in$ $\widehat{\operatorname{Diff}}(\mathbb{C}, 0)$ tel que $\hat{\varphi}_{*} \hat{X}=X_{0}$ et par suite

$$
\hat{\varphi}_{*} f_{0}=\hat{\varphi}_{*} \exp (\hat{X})=\exp \left(X_{0}\right)=f_{0}
$$

L'ensemble des champs de vecteurs formels $\hat{X}=\alpha z \partial_{z}+\cdots$ dont l'exponentielle est $f_{0}$ coïncide donc avec

$$
\left\{\hat{\varphi}^{*} \alpha z \partial_{z} ; \hat{\varphi}_{*} f_{0}=f_{0}\right\}
$$

D'après la Proposition 2.17, lorsque $a$ n'est pas périodique, $\hat{\varphi}$ est nécessairement linéaire et commute à $\alpha z \partial_{z}$, d'où l'unicité de $X$; lorsque $a$ est d'ordre $q$, on obtient $\hat{X}=\left(\alpha z \hat{g}\left(z^{q}\right)\right) \partial_{z}, g \in \mathbb{C}[[z]], g(0)=1$. Notamment, dans le cas $\alpha \in 2 i \pi \mathbb{Z}^{*}$, nous notons que $\exp \left(\alpha z \partial_{z}+\cdots\right)$ est l'identité, quels que soient les termes non linéaires du champ de vecteurs.

Si $\alpha=0$ et $f_{0}(z)=z$, d'après $(2.9)$, la seule possibilité est $\hat{X}=X_{0}=0$.

Supposons maintenant $f_{0}=a \exp \left(X_{p, \lambda}\right)=\exp (\hat{X})$ avec $a \neq 1$; alors $\hat{X}$ est formellement linéarisable $(\alpha \neq 0)$ et par suite $f$ aussi : contradiction.

Enfin, si $f_{0}=\exp \left(X_{p, \lambda}\right)$ alors on raisonne comme dans le cas linéaire avec $X_{0}=X_{p, \lambda}$ et de nouveau, les difféomorphismes formels qui commutent à $f_{0}$ commutent aussi à $X_{0}$ ce qui prouve l'unicité.

En particulier, on peut définir sans ambiguïté l'itérée complexe d'un germe de difféomorphisme tangent à l'identité $f(z)=z+\cdots \in \widehat{\operatorname{Diff}}(\mathbb{C}, 0)$ non trivial par

$$
f^{\circ t}(z)=\exp (t \hat{X}) \quad \text { pour tout } \quad t \in \mathbb{C}
$$

où $\hat{X}$ est le logarithme formel de $f$, c'est-à-dire l'unique élément de $\widehat{\mathcal{X}}(\mathbb{C}, 0)$ satisfaisant $f=\exp (\hat{X})$. Ça n'a pas de sens pour un autre type de germe.

Remarque 2.19. On prendra garde que la coordonnée normalisant $\hat{\varphi}$ construite dans la Proposition 2.16 n'est que formelle même si $f \in \operatorname{Diff}(\mathbb{C}, 0)$ est convergent. Elle est divergente dans de nombreux cas comme nous le verrons plus loin. De la même manière, le logarithme formel d'un difféomorphisme $f(z)=z+\cdots \in \operatorname{Diff}(\mathbb{C}, 0)$ convergent et tangent à l'identité est en général divergent, ainsi que la plupart des itérées complexes. Ceci sera étudié en détails dans le chapitre 2.

Remarque 2.20. L'invariant formel de l'itérée $n^{\text {ème }} f^{\circ}$ de $f(z)=$ $\exp \left(X_{p, \lambda}\right)$ n'est pas $\lambda$ mais $\frac{\lambda}{n}$ (voir Remarque 2.5). En particulier, si $f$ est conjuguée à une de ses itérées $f^{\circ n}, n \in \mathbb{Z}^{*}, n \neq 1$, alors $\lambda=0$. 
Remarque 2.21. L'invariant formel $\lambda$ d'un difféomorphisme tangent à l'identité $f$ (quelquefois appelé résidu de $f$ ) est donné par la formule:

$$
\frac{\lambda}{2 i \pi}=\operatorname{Rés}_{0} \frac{d z}{f(z)-z}+\frac{p+1}{2} .
$$

\subsection{Normalisation formelle des sous-groupes résoluble de $\operatorname{Diff}(\mathbb{C}, 0)$}

Nous allons démontrer l'analogue discret du Théorème 2.6. La notion de sous-groupes de Lie de dimension finie de $\operatorname{Diff}(\mathbb{C}, 0)$, ou plus généralement de $\widehat{\operatorname{Diff}}(\mathbb{C}, 0)$, dépend de la topologie dont nous allons équiper ces derniers. Néanmoins, pour toute topologie raisonnable, un tel sous-groupe $G$ définira un sous-groupe de Lie $J^{k} G$ au sens classique dans $J^{k} \operatorname{Diff}(\mathbb{C}, 0)$ dont la dimension se stabilisera à partir d'un certain $k(G)$. Il est donc naturel de s'intéresser aux sous-groupes $G \subset \widehat{\operatorname{Diff}}(\mathbb{C}, 0)$ de détermination finie, c'està-dire dont les éléments sont déterminés par leur jet d'ordre $k$ pour un certain $k=k(G) \in \mathbb{N}$. En d'autres termes, on suppose que le seul élément tangent à l'identité à l'ordre $\geq k$ dans $G$ est l'identité.

Théorème 2.22. Soit $G \subset \widehat{\operatorname{Diff}}(\mathbb{C}, 0)$ un sous-groupe. Alors sont équivalents :

- $G$ est de détermination finie,

- $G$ est virtuellement résoluble,

- G est métabélien.

Dans ce cas, il existe $\hat{\varphi} \in \widehat{\operatorname{Diff}}(\mathbb{C}, 0)$ tel que $\hat{\varphi}_{*} G$ soit contenu dans l'un des modèles

$-\mathbb{L}:=\left\{f(z)=a z ; a \in \mathbb{C}^{*}\right\}$,

$-\mathbb{E}_{p, \lambda}:=\left\{f(z)=a \cdot \exp \left(t X_{p, \lambda}\right) ; a^{p}=1, t \in \mathbb{C}\right\}, \lambda \in \mathbb{C}, p \in \mathbb{N}^{*}$,

$-\mathbb{A}_{p}:=\left\{f(z)=a z /\left(1-b z^{p}\right)^{1 / p} ; a \in \mathbb{C}^{*}\right.$ et $\left.b \in \mathbb{C}\right\}, p \in \mathbb{N}^{*}$.

Rappelons qu'un groupe $G$ est résoluble si sa suite centrale dérivée est finie :

$$
G \supset G^{\prime} \supset G^{\prime \prime} \supset \cdots \supset G^{(n)}=\{\text { identité }\}
$$

où $G^{(k+1)}=\left[G^{(k)}, G^{(k)}\right]$ est le sous-groupe de $G^{(k)}$ engendré par les commutateurs; $G$ est métabélien lorsque $G^{\prime}=[G, G]$ est abélien. On dit $G$ est virtuellement résoluble s'il admet un sous-groupe résoluble d'indice fini.

Les 3 modèles de l'énoncé sont les sous-groupes de Lie maximaux de $\widehat{\operatorname{Diff}}(\mathbb{C}, 0)$ dont les algèbres de Lie respectives sont

$-\mathbb{C}\left\langle z \partial_{z}\right\rangle$,

- $\mathbb{C}\left\langle X_{p, \lambda}\right\rangle, \lambda \in \mathbb{C}, p \in \mathbb{N}^{*}$,

- $\mathbb{C}\left\langle z \partial_{z}, z^{p+1} \partial_{z}\right\rangle, p \in \mathbb{N}^{*}$.

(voir Proposition 2.6). Rappelons que $X_{p, \lambda}=\frac{z^{p+1}}{1+\frac{\lambda}{2 i \pi} z^{p}} \partial_{z}$. 
Démonstration. Étant donnés deux éléments $f, g \in \operatorname{Diff}(\mathbb{C}, 0)$, on définit leur commutateur par

$$
[f, g]:=f \circ g \circ f^{\circ(-1)} \circ g^{\circ(-1)} .
$$

Un calcul formel immédiat nous dit que tout commutateur est tangent à l'identité :

$$
[a z+\cdots, b z+\cdots]=z+\cdots
$$

et que le commutateur de deux automorphismes observant des contacts d'ordres distincts $0<p<q$ à l'identité est tangent à l'identité à un ordre strictement supérieur $p+q>q$ :

$$
\left[z+c z^{p+1}+\cdots, z+d z^{q+1}+\cdots\right]=z+(q-p) c d z^{p+q+1}+\cdots .
$$

Ainsi, s'il existe, dans le groupe $G$, deux contacts distincts à l'identité, alors on en construit une infinité en emboitant les commutateurs. Par construction, on en retrouve une infinité dans le premier groupe dérivé $G^{\prime}:=$ $[G, G] \subset \hat{G}$ puisqu'il est engendré par les commutateurs de $G$ et, par récurence, dans chacun des groupes dérivés successifs $G^{(n+1)}:=\left[G^{(n)}, G^{(n)}\right]$, $n \in \mathbb{N}$. En particulier, aucun des groupes dérivés n'est trivial :

$$
\forall n \in \mathbb{N}, G^{(n)} \neq\{\text { identité }\},
$$

et $G$ n'est ni résoluble, ni de détermination finie.

Examinons maintenant la situation où $G$ ne contient pas d'élément tangent à l'identité non trivial. Alors $G$ est abélien et la flêche :

$$
\rho: G \hookrightarrow \mathbb{C}^{*} ; f(z)=a z+\cdots \mapsto a
$$

est un homomorphisme injectif de groupes. Alors $G$ se linéarise formellement comme suit. Supposons $G$ linéaire à l'ordre $k$; alors la flêche

$$
\rho_{k}: f(z)=a z+b z^{k+1}+\cdots \mapsto \frac{a w+b}{a^{k+1}}
$$

définit un morphisme de groupes à valeurs dans le groupe affine $\operatorname{Aff}(\mathbb{C})$ de la droite. Notons que si $\rho_{k}(f)$ est une translation, alors $a^{k}=1$ et $f^{\circ k}=z+k b z^{k+1}+\cdots$ est tangent à l'identité à l'ordre $k$; par hypothèse, $f^{\circ k}=$ identité, $b=0$ et $\rho_{k}(f)=$ identité. Ainsi, l'image de $G$ ne contient pas de translation non triviale et, par suite, se linéarise après conjugaison par un élément $\varphi \in \operatorname{Aff}(\mathbb{C})$. Choisissons un élément $\tilde{\varphi}(z)=\alpha z+\beta z^{p+1} \in$ $\operatorname{Diff}(\mathbb{C}, 0)$ relevant $\varphi$ : par construction, $\tilde{\varphi}_{*} G$ a son image dans le sousgroupe linéaire de $\operatorname{Aff}(\mathbb{C})$ et est donc lui-même linéaire à l'ordre $k+1$. De proche en proche, on linéarise $G$. En particulier, $G$ est de détermination finie. 
Supposons enfin qu'il existe un entier $p \in \mathbb{N}^{*}$ tel que tout élément tangent à l'identité dans $G$ l'est précisément à l'ordre $p$ :

$$
\forall f \in \hat{G}, f(z)=z+\cdots \neq \text { identité } \Rightarrow f(z)=z+c z^{p+1}+\cdots, c \in \mathbb{C}^{*} .
$$

On peut appliquer l'algorithme de linéarisation précédent jusqu'à l'ordre $p$ : après changement de coordonnée (polynomiale), $G$ est linéaire à l'ordre $p$. Alors le morphisme de groupes

$$
\rho: G \rightarrow \operatorname{Aff}(\mathbb{C}) ; f(z)=a z+b z^{p+1}+\cdots \mapsto \frac{a w+b}{a^{p+1}}
$$

est injectif. En effet, si deux éléments distincts $f, g \in G$ avaient le même jet d'ordre $p+1$, alors $f \circ g^{\circ(-1)} \in G$ serait un élément non trivial et tangent à l'identité à l'ordre $\geq p+1$, ce que nous excluons ici. En particulier, $G$ est aussi de détermination finie. Par ailleurs, le noyau $\operatorname{ker}(\rho)$, constitué des éléments de la forme $f(z)=a z+0 z^{p+1}+\cdots, a^{p}=1$, est fini d'ordre $\leq p$ et commute à $G$. Ainsi, $G$ est une extension centrale d'un sous-groupe de $\operatorname{Aff}(\mathbb{C})$ et est par suite métabélien.

Le sous-groupe $G_{1} \subset G$ constitué des éléments tangents à l'identité est visiblement abélien. Redressons un élément non trivial $f \in G_{1}$ sur son modèle formel $f=\exp \left(X_{p, \lambda}\right)$ par la Proposition 2.16. En particulier, $G_{1} \subset \widehat{\operatorname{Cent}}(f)$ et on déduit de la Proposition 2.17 que $G_{1}$ est un sousgroupe du groupe à 1 paramètre

$$
G_{1} \subset\left\{\exp \left(t X_{p, \lambda}\right) ; t \in \mathbb{C}\right\} .
$$

Plus généralement, si $G$ est abélien, la même proposition permet de conclure à la seconde forme normale de l'énoncé. Si par contre $G$ n'est pas abélien, $G_{1}$ est un sous-groupe normal et tout élément $g \in G$ satisfait

$$
g^{*} \exp \left(X_{p, \lambda}\right)=\exp \left(t \cdot X_{p, \lambda}\right)
$$

pour un $t=t_{g} \in \mathbb{C}$ convenable. Par conséquent, $g^{*} X_{p, \lambda}$ et $t . X_{p, \lambda}$ ont même image par l'application exponentielle la Proposition 2.18 entraine que

$$
g^{*} X_{p, \lambda}=t . X_{p, \lambda} .
$$

En repassant à la 1-forme duale comme dans la preuve de la Proposition 2.3 , on déduit que

$$
g^{*}\left(\frac{d z}{z^{p+1}}+\frac{\lambda}{2 i \pi} \frac{d z}{z}\right)=t\left(\frac{d z}{z^{p+1}}+\frac{\lambda}{2 i \pi} \frac{d z}{z}\right) ;
$$

si $G$ n'est pas abélien, alors on peut trouver $g$ ne commutant pas à $f$, c'est-à-dire pour lequel $t \neq 1$. En comparant les résidus des deux 1-formes, on déduit que $\lambda=0$, puis par intégration que $g(z)=a z /\left(1-t z^{p}\right)^{1 / p}$. 
Finalement, si un sous-groupe $G \subset \widehat{\operatorname{Diff}}(\mathbb{C}, 0)$ contient un sous-groupe résoluble $G_{0}$ d'indice fini, alors $G_{0}$ est de détermination finie ainsi que tous ses translatés $g \circ G_{0}$; puisque $G$ est union finie de translatés $g \circ G_{0}, G$ est aussi de détermination finie.

Corollaire 2.23. Soit $G$ un sous-groupe de $\widehat{\operatorname{Diff}}(\mathbb{C}, 0)$ et notons $G_{1}$ le sous-groupe des éléments tangents à l'identité. Si $G_{1}$ est réduit à l'identité, alors $G$ est formellement linéarisable.

Démonstration. C'est une conséquence immédiate de l'algorithme de linéarisation formelle développé dans la preuve du Théorème 2.22 : si l'algorithme bloque à l'ordre $k$, alors le morphisme

$$
\rho_{k}: f(z)=a z+b z^{k+1}+\cdots \mapsto \frac{a w+b}{a^{k+1}}
$$

envoie un certain élément $f \in G$ sur une translation non triviale. Alors $f^{\circ k}$ est un élément tangent à l'identité non trivial de $G$.

\subsection{Sous-groupes non résolubles de $\operatorname{Diff}(\mathbb{C}, 0)$ : quelques invariants}

Rappelons d'abord qu'une base de voisinages d'un élément $f \in \widehat{\operatorname{Diff}}(\mathbb{C}, 0)$ pour la topologie de Krull est donné par :

$$
\mathcal{U}_{k}(f)=\left\{g \in \widehat{\operatorname{Diff}}(\mathbb{C}, 0) ; J^{k} g=J^{k} f\right\}
$$

(l'ensemble des difféomorphismes qui coïncident avec $f$ à l'ordre $k$ ). Ces voisinages sont à la fois ouverts et fermés.

Étant donné un sous-groupe $G \subset \widehat{\operatorname{Diff}}(\mathbb{C}, 0)$, on définit :

$$
G_{k}=\left\{c \in \mathbb{C} ; \exists g=z+c z^{k+1}+\cdots \in G\right\} \quad \text { pour } \quad k \in \mathbb{N}^{*}
$$

et

$$
K(G)=\left\{k \in \mathbb{N}^{*} ; G_{k} \neq\{0\}\right\} .
$$

Les $G_{k}$ sont des groupes additifs, et à peu de choses près des invariants formels :

$$
\text { si } \varphi=a z+\cdots \in \widehat{\operatorname{Diff}}(\mathbb{C}, 0), \quad \text { alors }\left(\varphi^{*} G\right)_{k}=a^{k} \cdot G_{k} .
$$

La suite des contacts $K(G)$ de $G$ à l'identité est un invariant formel et à peu de choses près un semi-groupe :

$$
\text { si } k_{1}, k_{2} \in K(G) \quad \text { avec } \quad k_{1} \neq k_{2} \text {, }
$$

alors $k_{1}+k_{2} \in K(G)$ et $\left(k_{1}-k_{2}\right) \cdot G_{k_{1}} \cdot G_{k_{2}} \subset G_{k_{1}+k_{2}}$. 
En effet, si $g_{1}=z+c_{1} z^{k_{1}+1}+\cdots$ et $g_{2}=z+c_{2} z^{k_{2}+1}+\cdots$ réalisent $k_{1}, k_{2} \in K(G)$ dans $G$, alors $\left[g_{1}, g_{2}\right]=z+\left(k_{1}-k_{2}\right) c_{1} c_{2} z^{k_{1}+k_{2}+1}+\cdots$ réalise $k_{1}+k_{2} \in G$.

D'après le Théorème 2.22, sont équivalents :

- $G$ est résoluble

- $K$ a au plus un élément

- $G$ est discret pour la topologie de Krull.

Lorsque $G$ n'est pas résoluble, alors $K$ est infini et l'adhérence $\bar{G}$ de $G$ dans $\widehat{\operatorname{Diff}}(\mathbb{C}, 0)$ pour la topologie de Krull n'est pas dénombrable. En effet, choisissons une suite croissante $k_{1}<k_{2}<\cdots<k_{n}<\cdots$ d'éléments de $K(G)$ et pour chaque $n$ un élément $f_{n} \in G$ réalisant $k_{n} \in K(G)$. Alors pour toute suite $\left(\varepsilon_{n}\right)_{n}, \varepsilon_{n} \in\{0,1\}, f_{1}^{\varepsilon_{1}} \circ f_{2}^{\varepsilon_{2}} \circ \cdots \circ f_{n}^{\varepsilon_{n}} \circ \cdots$ est dans $\bar{G}$ : l'ensemble continu $\{0,1\}^{\mathbb{N}}$ s'injecte dans $\bar{G}$. En particulier, la donnée des $G_{k}$ est loin de donner une liste complète d'invariants formels pour $G: \bar{G}_{k}=G_{k}$ et pourtant $G \neq \bar{G}$ en général. Cependant, elle suffit à caractériser certaines propriétés de $G$ que nous utiliserons pour démontrer le Théorème de Nakai. Par exemple, le centralisateur formel de $G$

$$
\widehat{\operatorname{Cent}}(G)=\{f \in \widehat{\operatorname{Diff}}(\mathbb{C}, 0) ; f \circ g=g \circ f, \forall g \in G\}
$$

est un sous-groupe d'ordre fini de $\widehat{\operatorname{Diff}}(\mathbb{C}, 0)$; en effet, tout élément $f$ doit en particulier commuter avec des difféomorphismes tangents à l'identité à des ordres distincts et est par suite périodique d'après la Proposition 2.17 (d'ordre borné). Ainsi, dans une bonne coordonnée formelle, il est engendré par la rotation $f(z)=e^{2 i \pi / q} z$ et tout élément de $G$ est de la forme $g(z)=z \tilde{g}\left(z^{q}\right)$ : par conséquent, $K(G) \subset q \mathbb{Z}$.

Proposition 2.24 ([119]). Le centralisateur formel d'un sous-groupe non résoluble $G \subset \widehat{\operatorname{Diff}}(\mathbb{C}, 0)$ est d'ordre $d$, où $d$ est le diviseur commun aux éléments de $K(G)$. En particulier, dans une bonne coordonnée formelle, tous les éléments de $G$ sont de la forme $g(z)=z \tilde{g}\left(z^{d}\right)$.

Démonstration. Il suffit de montrer la dernière assertion; la construction de la coordonnée formelle est similaire à celle de la linéarisante formelle dans la preuve du Théorème 2.22. Supposons que les éléments de $G$ soient déjà de la forme :

$$
f(z)=a_{0} z+a_{d} z^{d+1}+\cdots+a_{k d} z^{k d+1}+a_{k d+l} z^{k d+l+1}+\cdots
$$

où $k, l \in \mathbb{N}^{*}, 0<l<k$. Alors la flêche

$$
f \mapsto \frac{a_{0} w+a_{k d+l}}{\left(a_{0}\right)^{k d+l}}
$$

définit un morphisme $\rho$ de $G$ dans le groupe affine Aff $(\mathbb{C})$. L'hypothèse $k d+l \notin K(G)$ signifie que $\rho(G)$ ne contient pas de translation non triviale. 
Alors $\rho(G)$ devient linéaire après conjugaison par une translation $w \mapsto w+t$ convenable. Après conjugaison de $G \operatorname{par} \varphi_{k d+l}(z)=z+(t z)^{k d+l+1}$, on peut supposer $\rho(G)$ linéaire et le coefficient de $z^{k d+l+1}$ est maintenant trivial pour tous les éléments de $G$. On itère ce procédé.

Par ailleurs, si $G \subset \operatorname{Diff}(\mathbb{R}, 0)$ est réel, alors $G_{k} \subset \mathbb{R}^{*}$ pour tout $k \in$ $K(G)$. Cette propriété n'est pas stable par changement de coordonnées, mais chaque $G_{k}$ reste contenu dans une droite réelle $L_{k} \subset \mathbb{C}$.

Théorème 2.25 ([119]). Un sous-groupe non résoluble $G \subset \widehat{\operatorname{Diff}}(\mathbb{C}, 0)$ est formellement conjugué à un sous-groupe de $\operatorname{Diff}(\mathbb{R}, 0)$ si, et seulement si, chaque $G_{k}$ est contenu dans une droite réelle $L_{k} \subset \mathbb{C}$. De plus, si d est le plus grand diviseur de $K(G)$, alors il existe une coordonnée formelle dans laquelle tout élément de $G$ est de la forme $g(z)=z \tilde{g}\left(z^{d}\right)$ avec $\tilde{g} \in \mathbb{R}[[x]]$.

Lemme 2.26. Soient $f, g \in \widehat{\operatorname{Diff}}(\mathbb{C}, 0)$ avec $f(z)=z+a_{p+1} z^{p+1}+\cdots$ réel et $g(z)=b_{1} z+\cdots$ quelconque. Alors sont équivalents :

1. $g^{*} f=g^{\circ(-1)} \circ f \circ g$ est réel,

2. $\left[g^{\circ(-1)}, f\right]$ est réel,

3. $g=\tilde{f} \circ \tilde{g}$ ò̀ $\tilde{f}$ commute à $f$ et $\tilde{g}$ est réel.

Démonstration. Les implications $1 \Leftrightarrow 2 \Leftarrow 3$ sont immédiates. Montrons que $2 \Rightarrow 3$. Remarquons d'abord que $\left[g^{\circ(-1)}, f\right]=z+\left(b_{1}^{p}-1\right) z^{p+1}+\cdots$ et donc, quitte à composer $g$ par un élément du centralisateur de $f, b_{1}$ est réel. Supposons $g$ non réel et écrivons $g=\tilde{g} \circ \tilde{f}$ où $\tilde{g}$ est réel et $\tilde{f}(z)=$ $z+b_{q+1} z^{q+1}+\cdots$ avec $b_{q+1}$ non réel. Dire que $g^{*} f=\tilde{f}^{*}\left(\tilde{g}^{*} f\right)$ est réel, c'est encore dire que $\left[\tilde{f}^{\circ}(-1), \tilde{g}^{*} f\right]=z+(q-p) a_{p+1} b_{q+1} z^{p+q+1}+\cdots$ est réel ce qui n'est possible que si $p=q$; mais dans ce cas, on peut de nouveau modifier $g$ par un élément du centralisateur de $f$.

Le Lemme technique suivant m'a été communiqué par Jean Écalle :

Lemme 2.27 (Écalle). Soient

$$
f(z)=z+a_{p+1} z^{p+1}+\cdots \quad \text { et } \quad g(z)=z+b_{q+1} z^{q+1}+\cdots
$$

deux éléments tangents à l'identité de $\widehat{\operatorname{Diff}}(\mathbb{C}, 0)$ avec $p \neq q$. Considérons

$$
h_{1}(z)=[g,[f,[f,[f, g]]]] \quad \text { et } \quad h_{2}(z)=[f,[g,[f,[f, g]]]] \text {. }
$$

Alors

$$
\left\{\begin{array}{l}
h_{1}(z)=z+m_{1} a_{p}^{3} b_{q}^{2} z^{3 p+2 q+1}+\cdots \\
h_{2}(z)=z+m_{2} a_{p}^{3} b_{q}^{2} z^{3 p+2 q+1}+\cdots
\end{array} \text { avec } \quad \frac{m_{1}}{m_{2}}=\frac{3 p+3 q}{2 p+4 q} .\right.
$$


Maintenant, posons $h=h_{1}^{\circ(2 p+4 q)} \circ h_{2}^{\circ(-3 p-3 q)}$. Alors

$$
h(z)=z+\sum_{k \geq 0} c_{3 p+2 q+k+1} z^{3 p+2 q+k+1}
$$

avec :

$$
c_{3 p+2 q+k+1}=\Delta(p, q, k)\left\{(k-p) a_{p}^{3} b_{q} b_{q+k}-(k-q) a_{p}^{2} a_{p+k} b_{q}^{2}\right\}
$$

modulo $\mathbb{Z}\left[a_{p}, \ldots, a_{p+k-1}, b_{q}, \ldots, b_{q+k-1}\right]$ et $\Delta(p, q, k) \neq 0$ pour $k>0$.

Démonstration. C'est un calcul immédiat qui se fait plus facilement au niveau de l'algèbre de Lie associée. D'après le Corollaire 2.18, on peut écrire :

$$
X:=\log f=\left(a_{p} z^{p+1}+\cdots\right) \partial_{z} \quad \text { et } \quad Y:=\log g=\left(b_{q} z^{q+1}+\cdots\right) \partial_{z} .
$$

On a alors

$$
\begin{aligned}
& {[\log [g,[f,[f,[f, g]]]]=[Y,[X,[X,[X, Y]]]]+\cdots,} \\
& \log [f,[g,[f,[f, g]]]]=[X,[Y,[X,[X, Y]]]]+\cdots
\end{aligned}
$$

En fait, les crochets $[Y,[X,[X,[X, Y]]]]$ et $[X,[Y,[X,[X, Y]]]]$ ont la particularité d'être tous les deux de même poids en $X$ et en $Y$ et, pour autant, indépendants (rappelons par exemple que $[Y,[X,[X, Y]]]=[X,[Y,[X, Y]]]$ ). Notons que si $X_{l}=z^{p_{l}+1} \partial_{z}$ pour $l=1, \ldots, k$, alors

$$
\left[X_{k},\left[X_{k-1}, \ldots\left[X_{3},\left[X_{2}, X_{1}\right]\right] \cdots\right]\right]=C_{\left(p_{k}, \ldots, p_{2}, p_{1}\right)} z^{p_{1}+p_{2}+\cdots p_{k}} \partial_{z}
$$

où

$$
C_{\left(p_{k}, \ldots, p_{2}, p_{1}\right)}=\left(p_{1}-p_{2}\right)\left(p_{1}+p_{2}-p_{3}\right) \cdots\left(p_{1}+p_{2}+\cdots+p_{k-1}-p_{k}\right) .
$$

Le coefficient de $z^{3 p+2 q+k+1}$ dans le crochet itéré $[Y,[X,[X,[X, Y]]]], k>0$, est un polynôme en les variables $a_{p}, \ldots, a_{p+k}, b_{q}, \ldots, b_{q+k}$ à coefficients dans $\mathbb{Z}$. Les monômes dans lesquels les variables $a_{p+k}$ et $b_{q+k}$ apparaissent sont :

$$
\begin{gathered}
\left(C_{(q, p, p, p+k, q)}+C_{(q, p, p+k, p, q)}+C_{(q, p+k, p, p, q)}\right) a_{p}^{2} a_{p+k} b_{q}^{2} \\
\text { et } \quad\left(C_{(q, p, p, p, q+k)}+C_{(q+k, p, p, p, q)}\right) a_{p}^{3} b_{q} b_{q+k} .
\end{gathered}
$$

On obtient des identités similaires pour $[X,[Y,[X,[X, Y]]]]$ et $\Delta(p, q, k)$ est à un facteur près l'un ou l'autre des déterminants

$$
\left|\begin{array}{ll}
C_{(q, p, p, p, q)} & C_{(q, p, p, p, q+k)}+C_{(q+k, p, p, p, q)} \\
C_{(p, q, p, p, q)} & C_{(p, q, p, p, q+k)}+C_{(p, q+k, p, p, q)}
\end{array}\right|
$$

$\mathrm{Ou}$

$$
\left|\begin{array}{ll}
C_{(q, p, p, p, q)} & C_{(q, p, p, p+k, q)}+C_{(q, p, p+k, p, q)}+C_{(q, p+k, p, p, q)} \\
C_{(p, q, p, p, q)} & C_{(p, q, p, p+k, q)}+C_{(p, q, p+k, p, q)}+C_{(p+k, q, p, p, q)}
\end{array}\right|
$$

On obtient $\Delta(p, q, k)=(q-p) k\left(k^{2}+k p+6 p q\right)$ qui est visiblement non nul sous nos hypothèses. 
Lemme 2.28. Soient $f(z)=z+a_{p+1} z^{p+1}+\cdots$ et $g(z)=z+b_{q+1} z^{q+1}+\cdots$ deux éléments tangents à l'identité de $G$. Si tous les $G_{k}$ sont réels et si les $p$ premiers coefficients (significatifs) $a_{p+1}, \ldots, a_{2 p}$ de $f$ sont réels, alors les $p$ premiers coefficients $b_{q+1}, \ldots, b_{q+p}$ de $g$ le sont aussi.

Démonstration. Supposons d'abord $p \neq q$; par hypothèse, $b_{q+1}$ est réel. Le Lemme 2.27 appliqué à $f$ et $g$ nous dit d'une part que $b_{q+2}$ est lui aussi réel et d'autre part que les $b_{q+3}, \ldots, b_{q+p}$ sont réels si, et seulement si, les $p-1$ premiers coefficients $c_{3 p+2 q+2}, \ldots, c_{3 p+2 q+p}$ de $h$ le sont. En remplaçant $g$ par $h$ et en réappliquant le Lemme 2.27 , on amorce une récurrence qui nous permet de conclure.

Maintenant, si $p=q$, le Lemme 2.26 nous dit que les $p$ premiers coefficients de $g$ sont réels si, et seulement si, les $p$ premiers coefficents de $[f, g]$ le sont, et on est ramené au cas précédent.

Démonstration du Théorème 2.25. Si $d>1$, on utilise d'abord la Proposition 2.24 pour se ramener au cas où tout élément de $G$ est de la forme $g(z)=z \tilde{g}\left(z^{d}\right)$. Alors $G$ est visiblement le relèvement par l'application $z \mapsto z^{d}$ d'un sous-groupe $\underline{G} \subset \widehat{\operatorname{Diff}}(\mathbb{C}, 0)$, l'élément $g(z)=z \tilde{g}\left(z^{d}\right)$ relevant $g(z):=z(\tilde{g}(z))^{d}$. On vérifie aisément que $\underline{G}$ n'est pas résoluble et satisfait aux hypothèses du Théorème avec $d=1$. Il suffit pour cela de construire une coordonnée formelle dans laquelle $\underline{G}$ est réel. On notera encore $G$ ce groupe dans toute la suite pour ne pas alourdir les notations.

Après conjugaison préliminaire par une homothétie, on peut supposer tous les $G_{k}$ réels. En effet, dès que le premier $G_{k}$ non trivial est redressé sur un sous-groupe de $\mathbb{R}$, la formule (2.11) nous dit que tous les autres le sont aussi. Notons que la partie linéaire de $G$ est nécessairement réelle.

Notons $G_{1}$ le sous-groupe des éléments tangents à l'identité de $G$ et choisissons un élément $f(z)=z+a_{p+1} z^{p+1}+\cdots$ non trivial de $G_{1}$. Plaçons nous dans une coordonnée formelle pour laquelle $f(z)=\exp \left(X_{p, \lambda}\right)=$ $z+z^{p+1}+\left(\frac{p+1}{2}-\frac{\lambda}{2 i \pi}\right) z^{2 p+1}+\cdots$ (voir Proposition 2.16) ; en particulier, $f$ est réel modulo $z^{2 p+1}$. Dans cette coordonnée, $G_{1}$ est réel modulo $z^{p+2}$ : si $g(z)=b_{1} z+\cdots$ est un élément de $G_{1}$, alors ses $p+1$ premiers coefficients sont réels. C'est une application directe du Lemme 2.26. Choisissons maintenant un élément $g(z)=z+b_{q} z^{q+1}+\cdots$ de $G_{1}$ avec $p<q$. Toujours d'après le Lemme 2.26, les $p$ premiers coefficients (significatifs) $b_{q+1}, \ldots, b_{q+p}$ de $g$ sont réels. Il existe, d'après la preuve de la Proposition 2.16 , un changement de coordonnée du type $\hat{\varphi}(z)=z+c z^{p+1}+\cdots$ pour lequel les $q$ premiers coefficients de $g$ deviennent réels : dans cette nouvelle coordonnée, $G_{1}$ est réel modulo $z^{q+2}$. De proche en proche, on construit une coordonnée dans laquelle $G_{1}$ est réel. Vérifions que $G$ est réel dans cette coordonnée.

Soit $h(z)=c_{0} z+\cdots$ un élément de $G$. Considérons $f(z)=z+a_{p} z^{p+1}+$ $\cdots$ et $g(z)=z+b_{q} z^{q+1}+\cdots$ dans $G_{1}$ avec $p$ et $q$ premiers entre eux. On peut trouver de tels éléments puisque nous nous sommes ramenés au 
cas $d=1$. Alors $f, g,\left[h^{\circ(-1)}, f\right]$ et $\left[h^{\circ(-1)}, g\right]$ sont réels (rappelons que $\left.[G, G] \subset G_{1}\right)$. Le Lemme 2.26 nous dit que

$$
h=\tilde{f} \circ \tilde{h}_{1}=\tilde{g} \circ \tilde{h}_{2}
$$

où $\tilde{f}$ commute avec $f, \tilde{g}$ commute avec $g$ et $\tilde{h}_{1}$ et $\tilde{h}_{2}$ sont réels. D'après le Lemme 2.28 et la Proposition 2.17, $c_{0}^{p}$ et $c_{0}^{q}$ sont réels, et donc $c_{0}$ l'est aussi. Ainsi, on peut supposer, quitte à modifier la décomposition $h=$ $\tilde{f} \circ \tilde{h}_{1}$, que $\tilde{f}$ est tangent à l'identité à l'ordre $p$ (Proposition 2.17) et, de la même manière, $\tilde{g}$ est tangent à l'identité à l'ordre $q$. Maintenant, si (par exemple) $p<q$, on déduit que le coefficient de $z^{p+1}$ dans $\tilde{f}$ est réel. Alors $\tilde{f}$ (Proposition 2.17) tout comme $h$ sont réels.

Remarque 2.29. On déduit facilement de l'étude précédente que deux éléments $f, g \in \widehat{\operatorname{Diff}}(\mathbb{C}, 0)$ (ou $f, g \in \operatorname{Diff}(\mathbb{C}, 0)$ ) génériques pour la topologie de Krull engendrent un sous-groupe $G \subset \widehat{\operatorname{Diff}}(\mathbb{C}, 0)$ non résoluble, non réel et pour lequel seul 1 est diviseur de $K$.

\subsection{Quelques problèmes ouverts}

C'est un problème encore ouvert que de déterminer le type de relations que peuvent satisfaire deux éléments de $\operatorname{Diff}(\mathbb{C}, 0)$ ou $\widehat{\operatorname{Diff}}(\mathbb{C}, 0)$ dès lors qu'ils n'engendrent pas un sous-groupe résoluble (dans ce dernier cas, le Théorème 2.22 nous ramène à l'étude des relations dans le groupe affine). L'énoncé suivant a été conjecturé dans [51]

Conjecture 2.30 (Alternative de Tits). Tout sous-groupe $G \subset \operatorname{Diff}(\mathbb{C}, 0)$ ou bien est résoluble, ou bien contient un sous-groupe libre d'indice fini.

On s'attend à ce qu'elle soit aussi vraie dans $\widehat{\operatorname{Diff}}(\mathbb{C}, 0)$ contrairement à la conjecture plus forte

Conjecture 2.31. Deux éléments $f, g \in \operatorname{Diff}(\mathbb{C}, 0)$ tangents à l'identité qui ne commutent pas engendrent un groupe libre.

L'analogue formel de cette dernière, auquel on a cru un temps, a été mis en défaut dans [72]. Dans cet article (voir aussi [153]), les auteurs construisent de nombreuses paires d'éléments de $\widehat{\operatorname{Diff}}(\mathbb{C}, 0)$ et de $\operatorname{Diff}(\mathbb{C}, 0)$ satisfaisant une relation non triviale et, pour autant, engendrant un groupe non résoluble. Cependant, dans le cas de deux difféomorphismes tangents à l'identité, les exemples obtenus sont divergents (voir [72], p.374). En outre, ces exemples sont sporadiques en ce sens que les paires $(f, g)$ satisfaisant une relation donnée sont déterminées par un jet fini à conjugaison près : il semble déraisonnable d'espérer construire une paire satisfaisant plusieurs relations indépendantes à la fois, ce qui conforte l'alternative de Tits (voir [78]). 
Voyons quelques exemples illustrant le type de difficultés, de résultats et de problèmes ouverts rencontrés.

Le premier groupe dérivé du groupe libre de rang $2,\langle a, b\rangle$, est le sous-groupe normal engendré par le commutateur $[a, b]$; un système libre et complet de générateurs est donné par :

$$
\left[a^{n}, b^{m}\right], \quad n, m \in \mathbb{Z}^{*}
$$

Ainsi, pour qu'un groupe $G=<f, g>$ soit métabélien, il faut et il suffit que tous les commutateurs ci-dessus commutent deux à deux, ce qui nécessite a priori une infinité de conditions. En fait, dans $\widehat{\operatorname{Diff}}(\mathbb{C}, 0)$, deux relations suffisent :

Proposition 2.32. Soit $G=<f, g>$ un sous-groupe de rang 2 de $\widehat{\operatorname{Diff}}(\mathbb{C}, 0)$. Alors $G$ est métabélien si, et seulement si, $\left[f, g^{\circ 2}\right]$ et $\left[f^{\circ 2}, g\right]$ commutent $\grave{a}[f, g]$.

Démonstration. D'après la Proposition 2.16, on peut trouver une coordonnée formelle dans laquelle $[f, g]=\exp \left(X_{p, \lambda}\right)$.

Puisque $\left[f^{\circ 2}, g\right]=f \circ[f, g] \circ f^{\circ}(-1) \circ[f, g]$, on obtient que $f \circ[f, g] \circ f^{\circ}(-1)$ commute à $[f, g]$. D'après la Proposition 2.17, il vient

$$
f^{*}[f, g]=f^{*} \exp \left(X_{p, \lambda}\right)=\exp \left(t X_{p, \lambda}\right), \quad t \in \mathbb{C}^{*} .
$$

Alors ou bien $t=1$ et $f=e^{2 i \pi \frac{k}{p}} \exp \left(s X_{p, \lambda}\right)$ (toujours la Proposition 2.17), ou bien $t \neq 1$ et, en raisonnant comme dans la preuve du Théorème 2.22, on déduit que $f=a \cdot \exp \left(s X_{p, \lambda}\right)$ (et $\left.\lambda=0\right)$. La commutation de $\left[f, g^{\circ 2}\right]$ avec $[f, g]$ entraine de la même manière que $g=b \cdot \exp \left(r X_{p, \lambda}\right)$; le groupe $G$ est résoluble.

Le premier exemple de relation non triviale dans un sous-groupe non résoluble de $\widehat{\operatorname{Diff}}(\mathbb{C}, 0)$ m'avait été communiqué par Jean Écalle en 1995 :

Proposition 2.33 (Écalle-Vallet). Pour tous $p, q \in \mathbb{N}^{*}, p<q$, il existe une unique paire de difféomorphismes formels

$f(z)=z+z^{p+1}+\cdots \in \widehat{\operatorname{Diff}}(\mathbb{C}, 0) \quad$ et $\quad g(z)=z+z^{q+1}+\cdots \in \widehat{\operatorname{Diff}}(\mathbb{C}, 0)$,

modulo conjugaison dans $\widehat{\operatorname{Diff}}(\mathbb{C}, 0)$ satisfaisant

$$
[g,[f,[f,[f, g]]]]^{\circ(2 p+4 q)}=[f,[g,[f,[f, g]]]]^{\circ(3 p+3 q)} .
$$

Démonstration. La relation de l'énoncé est satisfaite si, et seulement si, tous les coefficients $c_{3 p+2 q+k+1}$ du Lemme $2.27, k>0$, sont nuls. Une fois fixé $f \in \widehat{\operatorname{Diff}}(\mathbb{C}, 0)$, la formule pour $c_{3 p+2 q+k+1}$ nous montre que le coefficient $b_{q+k}$ de $g$ est déterminé par le coefficient $a_{p+k}$ de $f$ et des 
précédents excepté lorsque $k=p$ : dans ce cas, une condition apparaît sur le coefficient $a_{2 p}$, fixant par là la classe formelle de $f$, et le coefficient $b_{q+p}$ est libre; cette liberté vient de la possibilité de conjuguer $g$ par un élément du centralisateur de $f$.

On peut construire des sous-groupes libres de $\operatorname{Diff}(\mathbb{C}, 0)$ de la manière suivante (voir [13]). D'après [62], le groupe de germes de transformations complexes engendré en $\left(\mathbb{R}^{+}, \infty\right)$ par les deux groupes abéliens

$$
P=\left\{w \mapsto w^{\frac{p}{q}} ; \frac{p}{q} \in \mathbb{Q}_{>0}\right\} \quad \text { et } \quad T=\{w \mapsto w+t ; t \in \mathbb{C}\}
$$

(où la racine réelle positive est choisie sur $\mathbb{R}^{+}$) est le produit libre de $P$ et de $T$. Notons que ce résultat est non trivial, obtenu après soixante pages de théorie de Galois. En relevant $T$ par $z \mapsto \frac{1}{z^{p}}, p \in \mathbb{N}^{*}$, on retrouve le groupe à 1 paramètre

$$
G_{p}=\left\{f(z)=z /\left(1-t z^{p}\right)^{1 / p} ; t \in \mathbb{C}\right\} .
$$

En particulier, le résultat de S.D. Cohen nous dit que le groupe engendré par $G_{p}$ et $G_{q}$ dans $\operatorname{Diff}(\mathbb{C}, 0)$ est le produit libre de ces deux sous-groupes dès que $p \neq q$. En particulier

$$
f(z)=z /\left(1-z^{p}\right)^{1 / p} \quad \text { et } \quad g(z)=z /\left(1-z^{q}\right)^{1 / q}
$$

engendrent un groupe libre de rang 2. On en déduit aisément que l'ensemble des représentations fidèles est dense dans l'ensemble des représentations du groupe libre $\mathbb{F}_{2}=\langle a, b>$ de rang 2 dans le sens suivant : toute autre représentation

$$
\mathbb{F}_{2}=<a, b>\rightarrow \operatorname{Diff}(\mathbb{C}, 0) ;\left\{\begin{array}{l}
a \mapsto \tilde{f} \\
b \mapsto \tilde{g}
\end{array}\right.
$$

est approchée (par exemple au sens d'une quelconque des topologies de la Remarque 2.8) par une suite de représentations fidèles. En effet, il suffit pour cela de considérer la famille de représentations définies par

$$
\rho_{t}:\left\{\begin{array}{l}
a \mapsto t \tilde{f}+(1-t) f \\
b \mapsto t \tilde{g}+(1-t) g
\end{array} \quad t \in \mathbb{C} ;\right.
$$

excepté pour peut-être deux valeurs de $t, \rho_{t}$ est bien à valeurs dans $\operatorname{Diff}(\mathbb{C}, 0)$. Pour chaque élément non trivial $m \in \mathbb{F}_{2}$, l'ensemble des $t$ pour lesquels $\rho_{t}(m) \neq$ identité est un ouvert de Zariski non vide puisqu'il contient $t=0$. Comme $\mathbb{F}_{2}$ est dénombrable, l'ensemble des $t \in \mathbb{C}$ pour lesquels $\rho_{t}$ est fidèle est le complémentaire d'une union dénombrable de points.

Dans [56], les arguments précédents sont réutilisés pour construire une représentation fidèle de

$$
\operatorname{PSL}(2, \mathbb{Z})=<a, b \mid a^{2}=b^{3}>.
$$


Plus généralement, si $p, q \in \mathbb{N}^{*}$ premiers entre eux alors la représentation

$$
<a, b \mid a^{p}=b^{q}>\rightarrow \operatorname{Diff}(\mathbb{C}, 0) ;\left\{\begin{array}{l}
a \mapsto f(z)=e^{2 i \pi / p} z /\left(1-z^{q}\right)^{1 / q} \\
b \mapsto g(z)=e^{2 i \pi / q} z /\left(1-z^{p}\right)^{1 / p}
\end{array}\right.
$$

est fidèle. En effet, il est facile de voir sur la partie linéaire qu'un mot est non trivial dès qu'il n'est pas dans le premier groupe dérivé. Par ailleurs, ce dernier est engendré par les $(p-1)(q-1)$ commutateurs

$$
\left[f^{k}, g^{l}\right]=\left[z /\left(1-\lambda_{(l-k+1, l)} z^{q}\right)^{1 / q}, z /\left(1-\lambda_{(-k, l-k-1)} z^{p}\right)^{1 / p}\right],
$$

where

$$
\left\{\begin{array}{l}
k=1, \ldots, p-1 \\
l=1, \ldots, q-1
\end{array}\right.
$$

où

$$
\lambda_{n, n+m}=\lambda^{n}+\lambda^{n+1}+\cdots+\lambda^{n+m}, \lambda=e^{2 i \pi p / q} .
$$

Le résultat de Cohen permet encore de conclure à l'absence de relation entre ces commutateurs.

Les groupes de triangles

$$
\Gamma_{p, q, r}=<a, b, c \mid a^{p}=b^{q}=c^{r}=a b c=1>
$$

admettent des représentations fidèles

- dans le groupe affine Aff($(\mathbb{C})$ lorsque $\frac{1}{p}+\frac{1}{q}+\frac{1}{r}=1$,

- dans le groupe PGL(2, $\mathbb{C})$ lorsque $\frac{1}{p}+\frac{1}{q}+\frac{1}{r}<1$.

Dans le premier cas, on en déduit des représentations fidèles dans $\operatorname{Diff}(\mathbb{C}, 0)$ en composant par le plongement

$$
\operatorname{Aff}(\mathbb{C}) \rightarrow \operatorname{Diff}(\mathbb{C}, 0) ; a w+b \mapsto f(z)=z /(a+b z) .
$$

Par contre, dans le cas hyperbolique $\frac{1}{p}+\frac{1}{q}+\frac{1}{r}<1$, on ne connait pas de représentation fidèle du groupe $\Gamma_{p, q, r}$ dans $\operatorname{Diff}(\mathbb{C}, 0)$ (ni même dans $\widehat{\operatorname{Diff}}(\mathbb{C}, 0))$. Bien sûr, une obstruction apparait au niveau du terme linéaire : lorsque $p$ et $q$ sont premiers entre eux, par exemple, le produit $f \circ g$ de deux germes de difféomorphismes d'ordre $p$ et $q$ ne peut être que d'ordre $p q($ ou $\infty)$ à cause de sa partie linéaire. Outre cette obstruction, c'est un bon exercice (quoiqu'un peu fastidieux) que de vérifier que l'espace des modules de telles représentations

$$
\Gamma_{p, q, r} \rightarrow \widehat{\operatorname{Diff}}(\mathbb{C}, 0)
$$

modulo changements de coordonnée formelle est de dimension infinie. Grosso-modo, la périodicité d'un germe $f$ dont la partie linéaire est d'ordre $p$ impose une condition tous les $p$ coefficients ; la condition d'hyperbolicité $\frac{1}{p}+\frac{1}{q}+\frac{1}{r}<1$ laisse une infinité de coefficients libres de toute condition. 
Il est affirmé dans [72] que cette liberté permet de construire des exemples convergents, et même que l'espace des modules des représentations

$$
\Gamma_{p, q, r} \rightarrow \operatorname{Diff}(\mathbb{C}, 0)
$$

est encore de dimension infinie. Ici, on considère les représentations modulo conjugaison formelle ou analytique, ça revient au même (voir Théorème 3.36). Il semble raisonnable d'espérer qu'elles soient fidèles en général. Notons à ce propos que le premier groupe dérivé de $\Gamma_{2,5,10}$ est le groupe fondamental $\pi_{1}\left(\Sigma_{2}\right)$ de la surface de genre 2 (comme nous l'apprend la théorie des revêtements ramifiés); l'existence d'une représentation fidèle de ce dernier dans $\operatorname{Diff}(\mathbb{R}, 0)$ est une vieille question d'É. Ghys encore ouverte à ma connaissance. Récemment, l'existence de représentations fidèles des groupes de surfaces

$$
\pi_{1}\left(\Sigma_{g}\right) \hookrightarrow \operatorname{Diff}(\mathbb{R}, 0)
$$

a été démontrée par Cantat, Cerveau, Guirardel et Souto pour $g \geq 4$ (voir [45]).

Notons pour finir que l'on ne sait pas décrire l'espace des modules des représentations du groupe modulaire

$$
\operatorname{PSL}(2, \mathbb{Z}) \simeq \Gamma_{2,3, \infty}=<a, b ; a^{2}=b^{3}=1>
$$

dans $\operatorname{Diff}(\mathbb{C}, 0)$. Ce dernier problème est équivalent à la classification des singularités nilpotentes génériques des feuilletages holomorphes (voir [58]). Tout comme dans [118], on peut construire des formes normales formelles uniques pour de telles représentations, mais elles seront divergentes en général : on ne sait pas reconnaitre quelle forme normale formelle (divergente) provient d'une représentation convergente. Ce problème résiste lui aussi depuis bientôt 30 ans. 


\section{Chapitre 3}

\section{Classification analytique}

On dira que deux éléments $f, g \in \operatorname{Diff}(\mathbb{C}, 0)$ sont analytiquement conjugués s'il existe $\varphi \in \operatorname{Diff}(\mathbb{C}, 0)$ tel que $g=\varphi_{*} f=\varphi \circ f \circ \varphi^{\circ(-1)}$, c'est-à-dire $\varphi \circ f=g \circ \varphi$. Nous passons rapidement sur les problèmes de linéarisation et de petits diviseurs pour arriver rapidement aux difféomorphismes tangents à l'identité; ils jouent un rôle crucial dans notre étude. Après un survol historique, nous consacrons plusieurs sections à la construction de la liste complète des invariants analytiques. Nous en donnons quelques applications remarquables puis déduisons la classification analytique des sous-groupes résolubles de $\operatorname{Diff}(\mathbb{C}, 0)$ qui ne sont pas formellement linéarisables.

\subsection{Linéarisation et problèmes de petits divi- seurs}

Étant donné $f=a z+\cdots \in \operatorname{Diff}(\mathbb{C}, 0)$, rappelons qu'il existe $\hat{\varphi} \in$ $\widehat{\operatorname{Diff}}(\mathbb{C}, 0)$ tel que $\hat{\varphi}_{*} f(z)=a z$ dans les cas suivants (voir Proposition 2.16)

- $a$ et $f$ sont périodiques (d'ordre fini),

- $a$ n'est pas périodique (et donc $f$ non plus).

Débarrassons nous rapidement du premier cas :

Proposition 3.1. Tout élément $f \in \operatorname{Diff}(\mathbb{C}, 0)$ d'ordre fini est analytiquement linéarisable : il existe $\varphi \in \operatorname{Diff}(\mathbb{C}, 0)$ tel que $\varphi \circ f=f^{\prime}(0) \varphi$.

Démonstration. On vérifie sans peine que le difféomorphisme tangent à l'identité défini par $\varphi:=\frac{1}{n} \sum_{k=0}^{n-1} \frac{1}{\left(f^{\prime}(0)\right)^{k}} f^{\circ k}$ satisfait l'égalité.

Profitons-en pour déduire immédiatement le : 
Corollaire 3.2. Si $G \subset \operatorname{Diff}(\mathbb{C}, 0)$ est un sous-groupe d'ordre fini, alors il est analytiquement linéarisable : il existe $\varphi \in \operatorname{Diff}(\mathbb{C}, 0)$ tel que

$$
\varphi_{*} G:=\left\{\varphi \circ f \circ \varphi^{\circ(-1)} ; f \in G\right\}
$$

est un sous-groupe du groupe linéaire

$$
\mathbb{L}:=\left\{f(z)=a z ; a \in \mathbb{C}^{*}\right\} .
$$

Démonstration. Sous nos hypothèses, le morphisme $G \rightarrow \mathbb{C}^{*}$ défini par $f \mapsto f^{\prime}(0)$ est injectif : sinon, il existerait un élément tangent à l'identité non trivial dans $G$, donc d'ordre infini, contradiction. Il s'en suit que $G$ est cyclique; il suffit alors de linéariser un générateur de $G$. La linéarisante tangente à l'identité est a posteriori donnée par $\varphi:=\frac{1}{\# G} \sum_{f \in G} \frac{1}{f^{\prime}(0)} f$ où \#G désigne l'ordre de $G$.

Dans toute la suite, nous supposerons $f(z)=a z+\cdots$ avec $a$ non périodique. Nous cherchons donc une coordonnée $\varphi \in \operatorname{Diff}(\mathbb{C}, 0)$ satisfaisant à l'équation fonctionnelle de Schröder :

$$
\varphi \circ f=a \varphi
$$

D'après la Proposition 2.17, toute autre solution sera de la forme $\hat{\varphi}=b \varphi$ et donc convergente aussi.

Ce problème a été étudié par le mathématicien allemand Ernst Schröder en 1870 (voir [200]) ; il montre que 0 est un point fixe attractif sur un voisinage dès que $|a|<1$. Le premier résultat de linéarisation a été démontré par le mathématicien français Gabriel Kœnigs en 1884 (voir [101]) puis généralisé en toute dimension par H. Poincaré.

Théorème 3.3 (Kœenigs). Si $f(z)=a z+\cdots \in \operatorname{Diff}(\mathbb{C}, 0)$ avec $|a| \neq 1$, alors $f$ est analytiquement linéarisable (et toute linéarisante formelle $\hat{\varphi}$ converge).

Démonstration. Quitte à considérer $f^{\circ(-1)}$ plutôt que $f$, on suppose $|a|<$ 1. Nous allons construire $\varphi$ comme limite des moyennes partielles

$$
\varphi_{n}:=\frac{1}{n} \sum_{k=0}^{n} \frac{1}{a^{k}} f^{\circ k} .
$$

Commençons par majorer la partie non linéaire de $f$. L'expression $1-\frac{a z}{f(z)}$ est analytique et s'annule en 0 de sorte que, sur un disque $\mathbb{D}_{r}$ de rayon $r>0$ suffisamment petit centré en 0 , elle est majorée en module par $b|z|$ pour un $b>0$ convenable. Par suite, il vient :

$$
|f(z)| \leq \frac{|a z|}{1-b|z|}
$$


pour tout $z \in \mathbb{D}_{r}$. En particulier, on déduit d'ores-et-déjà que $f$ est contractante sur $\mathbb{D}_{r}$ pourvu que $b<\frac{1-|a|}{b}$ : ses itérées positives $f^{\circ n}$ sont donc toutes bien définies sur $\mathbb{D}_{r}$. En itérant le second membre de notre majoration (qui est une transformation de Mœbius), on obtient :

$$
\left|f^{\circ n}(z)\right| \leq \frac{\left|a^{n} z\right|}{1-b \frac{1-|a|^{n}}{1-|a|}|z|}
$$

de sorte que l'on obtient la majoration uniforme :

$$
\left|\frac{1}{a^{n}} f^{o n}(z)\right| \leq \frac{r}{1-\frac{b}{1-|a|} \cdot r}
$$

(toujours pour $z \in \mathbb{D}_{r}$ ). La famille des moyennes $\varphi_{n}$ admet la même borne uniforme et forme donc, d'après le Théorème de Montel, une famille normale sur le disque $\mathbb{D}_{r}$.

Montrons que toute valeur d'adhérence $\varphi$ est une linéarisante. On a

$$
\varphi_{n} \circ f-a \cdot \varphi_{n}=\frac{a}{n}\left(\frac{1}{a^{n}} f^{\circ n}-z\right) ;
$$

comme nous venons de le voir, le terme entre parenthèses est uniformément borné en module, et on obtient à la limite $\varphi \circ f-a \cdot \varphi=0$. Par unicité de la linéarisante tangente à l'identité, on déduit part ailleurs que toutes les valeurs d'adhérence $\varphi$ de la famille normale coïncident et donc que la suite $\varphi_{n}$ converge.

En raffinant les estimations de la preuve précédente, on peut montrer que la suite

$$
\varphi_{n}:=f_{0}^{\circ(-n)} \circ f^{\circ n}=\frac{1}{a^{n}} f^{\circ n} .
$$

converge elle-même vers une linéarisante (voir [146]).

Autre démonstration plus géométrique. Il est très facile de voir (supposant comme précédemment $|a|<1$ ) que 0 est point fixe attractif pour la dynamique : après majoration $|f(z)-a z|<C \cdot|z|^{2}$ par le Lemme de Schwarz, on déduit que $|f(z)|<(|a|+\varepsilon)|z|$ pour $\varepsilon>0$ arbitrairement petit, pourvu que $|z|<r=\frac{\varepsilon}{C}$. Par suite, $f^{\circ n}$ converge uniformément vers 0 sur le disque $\mathbb{D}_{r}$ de rayon $r$.

Le quotient du disque épointé $\mathbb{D}_{r}^{*}=\mathbb{D}_{r} \backslash\{0\}$ par $f$ est un tore complexe (de dimension 1) dont un domaine fondamental est $\mathbb{D}_{r}-f\left(\mathbb{D}_{r}\right)$ : il existe une application holomorphe

$$
\psi: \mathbb{D}_{r}^{*} \rightarrow \mathbb{C} / \mathbb{Z}+\tau \mathbb{Z}
$$




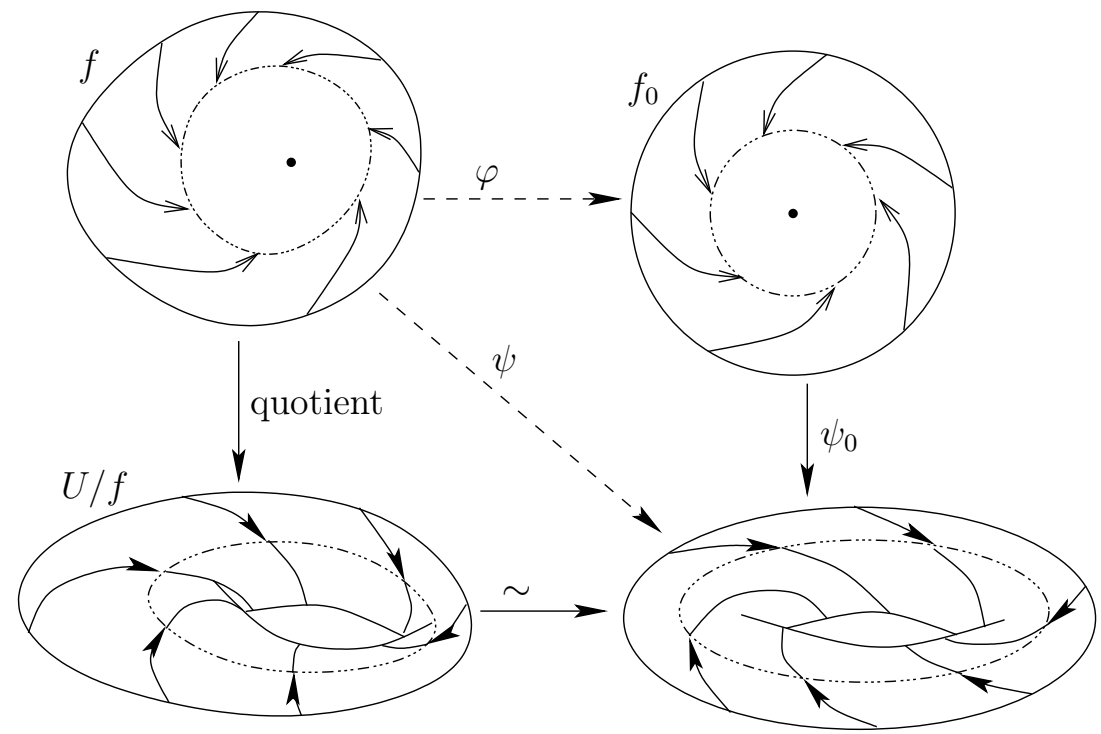

Figure 3.1 - Uniformisation de Kœnigs

envoyant le lacet fondamental $\partial \mathbb{D}_{r}$ sur la classe d'homotopie du lacet $[0,1]$ (on a choisi $\operatorname{Im} \tau>0$ ), satisfaisant $\psi \circ f=\psi$ et réalisant le quotient $\mathbb{D}_{r}^{*} / f$. Par ailleurs, l'application

$$
\psi_{0}: \mathbb{C}^{*} \rightarrow \mathbb{C} / \mathbb{Z}+\tau \mathbb{Z} ; z \mapsto \frac{1}{2 i \pi} \log (z)
$$

réalise le quotient de $\mathbb{C}^{*}$ par la contraction linéaire $f_{0}(z)=e^{2 i \pi \tau} z$. L'application

$$
\varphi=\psi_{0}^{\circ(-1)} \circ \psi: \mathbb{D}_{r}-f\left(\mathbb{D}_{r}\right) \rightarrow \mathbb{C}^{*}
$$

obtenue après avoir choisi une détermination de $\psi_{0}^{\circ(-1)}$ sur le domaine fondamental $\psi\left(\mathbb{D}_{r}-f\left(\mathbb{D}_{r}\right)\right)$ se prolonge analytiquement sur $\mathbb{D}_{r}^{*}$ par :

$$
\varphi \circ f(z)=f_{0} \circ \varphi(z)
$$

et se prolonge par Riemann en $0: \varphi$ est la linéarisante cherchée. A posteriori, on note que $a=e^{2 i \pi \tau}$ puisque la partie linéaire est un invariant formel.

Les difficultés rencontrées lorsque $|a|=1$ ( $a$ non périodique) sont appelées problèmes de petits diviseurs : les coefficients de l'unique linéarisante 
tangente à l'identité $\hat{\varphi}=z+\sum_{n \geq 2} u_{n} z^{n}$ sont donnés par :

$$
u_{n+1}=\frac{1}{a^{n+1}-a}\left[a_{n+1}+\sum_{p=2}^{n} a_{p} \sum_{\substack{k_{1}+\cdots+k_{p}=n+1 \\ k_{i} \geq 1}} u_{k_{1}} \cdots u_{k_{p}}\right]
$$

où $f(z)=a z+\sum_{n \geq 2} a_{n} z^{n}$; les dénominateurs $a^{n+1}-a$ sont arbitrairement petits et donc source de divergence (voir Théorème 3.4 et sa preuve).

En 1917, G.A. Pfeiffer construit un exemple de dynamique non linéarisable (voir [172]) puis en 1928, H. Cremer montre qu'il en existe dès que le multiplicateur $a$ satisfait une certaine condition arithmétique (voir $[64,65])$. Afin de la décrire, écrivons $a=e^{2 i \pi \alpha}$ et rappelons ce qu'est le développement de $\alpha \in \mathbb{R}$ en fraction continue. On définit une suite d'entiers $\alpha_{n} \in \mathbb{N}^{*}$ (excepté $\alpha_{0} \in \mathbb{Z}$ ) et une suite de nombres $0 \leq r_{n}<1$ par :

$$
\alpha=\alpha_{0}+r_{0} \quad \text { et, pour tout } n \in \mathbb{N}, \frac{1}{r_{n}}=\alpha_{n+1}+r_{n+1}
$$

de sorte que :

$$
\alpha=\alpha_{0}+\frac{1}{\alpha_{1}+\frac{1}{\alpha_{2}+\frac{1}{\alpha_{3}+\cdots}}} .
$$

Si $\alpha$ est rationnel, l'algorithme s'arrête pour un certain $n \in \mathbb{N}\left(r_{n}=0\right)$ et $\alpha$ est donné par la $n^{\text {ème }}$ réduite :

$$
\frac{p_{n}}{q_{n}}=\alpha_{0}+\frac{1}{\alpha_{1}+\frac{1}{\alpha_{2} \cdots \frac{1}{\alpha_{n}}}} .
$$

Lorsque $\alpha$ n'est pas rationnel, les réduites $\frac{p_{n}}{q_{n}}$ convergent exponentiellement vite vers $\alpha$ (Voir [146], Appendix C). Plus précisément, la croissance des dénominateurs $q_{n}$ est au moins exponentielle (la suite $q_{n}$ est minorée par une suite géométrique dont la raison est le nombre d'or $\frac{1+\sqrt{5}}{2}$ ) et l'erreur $\left|\frac{p_{n}}{q_{n}}-\alpha\right|$ est de l'ordre de $\frac{1}{q_{n+1}}$. Géométriquement, la droite de pente $\alpha$ sépare l'ensemble des points entiers $(p, q) \in \mathbb{N}^{*} \times \mathbb{Z}$ en deux parties; leurs enveloppes convexes ont pour sommets (au voisinage de la droite) précisément les points $\left(p_{n}, q_{n}\right)$.

Théorème 3.4 (Cremer). Il existe $f(z)=a z+\cdots \in \operatorname{Diff}(\mathbb{C}, 0)$ non linéarisable dès que :

$$
\limsup _{n \geq 0} \frac{\log \left(q_{n+1}\right)}{q_{n}}=+\infty
$$


Démonstration. Il s'agit ici de construire $f$ de sorte que la linéarisante tangente à l'identité $\hat{\varphi}=z+\sum_{n \geq 2} u_{n} z^{n}$ diverge, c'est-à-dire que $\limsup _{n \geq 0} \frac{\log \left(u_{n}\right)}{n}=$ $+\infty$. Nous allons construire les coefficients $a_{n}$ de $f$ tous de module 1 afin d'être assuré de la convergence de $f$; maintenant, on fixe l'argument de $a_{n+1}$ égal à celui du deuxième terme de la somme entre crochets dans l'expression (3.1) de $u_{n+1}$ de sorte que :

$$
\left|u_{n+1}\right| \geq \frac{1}{|a|^{n+1}-|a|} .
$$

Maintenant, la sous-suite $\frac{\log \left(u_{q_{n}}\right)}{q_{n}}$ est minorée par une suite de l'ordre de $\frac{\log \left(q_{n+1}\right)}{q_{n}}$.

A contrario, C.L. Siegel donne dans [208] une conditions arithmétique sur $a$ sous laquelle la dynamique de $f$ est systématiquement linéarisable.

Théorème 3.5 (Siegel). Si $a=e^{2 i \pi \alpha}, \alpha \in \mathbb{R} \backslash \mathbb{Q}$, et s'il existe des constantes $C, M>0$ telles que:

$$
\left|\alpha-\frac{p}{q}\right|>\frac{C}{q^{M}},
$$

alors tout élément $f(z)=a z+\cdots \in \operatorname{Diff}(\mathbb{C}, 0)$ est analytiquement linéarisable.

De plus, l'ensemble des nombres a du cercle satisfaisant la condition précédente est de mesure totale pour Lebesgue.

La condition arithmétique de Siegel signifie précisément que log $\left|a^{n+1}-a\right|$ est en $O(\log n)$, i.e. les diviseurs $\left(a^{n+1}-a\right)$ apparaissant dans les coefficients de $\hat{\varphi}$ adhèrent lentement à 0 . Ainsi, la linéarisabilité reste générique lorsqu'on se restreint aux multiplicateurs $a$ de module 1 .

Une condition suffisante plus faible est donnée par A.D. Brjuno dans [21] :

Théorème 3.6 (Brjuno). Si $a=e^{2 i \pi \alpha}, \alpha \in \mathbb{R} \backslash \mathbb{Q}$, et si les réduites $\frac{p_{n}}{q_{n}}$ de $\alpha$ satisfont

$$
\text { (B) } \sum_{n \geq 0} \frac{\log q_{n+1}}{q_{n}}<\infty,
$$

alors tout élément $f(z)=a z+\cdots \in \operatorname{Diff}(\mathbb{C}, 0)$ est analytiquement linéarisable.

Nous noterons $\mathcal{B}$ l'ensemble des nombres $a \in \mathbb{S}^{1}$ satisfaisant à la condition de Brjuno. Jean-Christophe Yoccoz démontre en 1987 l'optimalité de cette condition dans $[229,230]$ : 
Théorème 3.7 (Yoccoz). Si $a=e^{2 i \pi \alpha}$ ne satisfait pas à la condition de Brjuno $(\mathcal{B})$, alors le polynôme quadratique $f(z)=a z(1-z)$ n'est pas analytiquement linéarisable : il possède une suite d'orbites périodiques accumulant 0 .

De plus, il existe une infinité non dénombrable de classes de conjugaisons analytique parmis les germes de difféomorphismes de la forme $f(z)=a z+$ $\cdots \in \operatorname{Diff}(\mathbb{C}, 0)$.

Nous donnerons dans la section 4.1.3 une description des dynamiques non linéarisables dûe à Ricardo Pérez-Marco.

Exemple 3.8. On note $f_{a}(z)=a z(1-z)$ le polynôme quadratique. Si a est une racine de l'unité, $f_{a}$ n'est certainement pas périodique car son itérée $n^{\grave{e} m e}$ est un polynôme de degré $2 n$. Si $a=e^{2 i \pi p / q}$, alors $f_{a}^{\circ q}(z)-$ $z$ a un zéro d'ordre $q+1$ à l'origine; en effet, on peut linéariser $f_{a}$ à l'ordre q (voir algorithme de la preuve de la proposition 2.16), et donc $f_{a}^{\circ q}$ aussi. D'après le théorème de Rouché, il existe un voisinage ouvert épointé $U(p / q, n)$ de $p / q$ dans $\mathbb{R}$ tel que, pour $\alpha \in U(p / q, n)$ et $a=e^{2 i \pi \alpha}$, le polynôme $f_{a}^{\circ q}(z)-z$ possède une racine simple à l'origine, et $q$ autres racines dans le disque de rayon $1 / n$. Ces $q$ racines correspondent à une orbite finie de longueur q pour $f_{a}$. La réunion $U_{n}$ des $U(p / q, n)$ lorsque $p / q$ décrit $\mathbb{Q}$ est un ouverte et dense; il s'ensuit que que l'intersection $\mathcal{U}$ des $U_{n}$ est dense et non dénombrable (théorème de Baire). Si $\alpha \in \mathcal{U}$ et n'est pas une racine de l'unité, alors $f_{a}$ n'est pas linéarisable car il possède des orbites finies arbitrairement proche de l'origine.

Corollaire 3.9. Soit $G$ un sous-groupe abélien de $\operatorname{Diff}(\mathbb{C}, 0)$. Si $G$ contient un élément $f(z)=a z+\cdots$ avec $|a| \neq 1$ ou encore $|a|=1$ et $a \in \mathcal{B}$, alors $G$ est analytiquement linéarisable.

Démonstration. Soit $\varphi \in \operatorname{Diff}(\mathbb{C}, 0)$ la linéarisante de $f$ donnée par le Théorème 3.3 ou $3.7: \varphi_{*} f(z)=a z$. D'après la Proposition 2.17, $\varphi_{*} G \subset$ $\widehat{\operatorname{Cent}}\left(\varphi_{*} f\right)$ est linéaire.

Remarque 3.10. Le Corollaire 2.18 permet toujours de plonger $f(z)=$ $a z+\cdots \in \operatorname{Diff}(\mathbb{C}, 0)$, lorsque $|a|=1$ n'est pas une racine de l'unité, dans le groupe à 1 paramètre formel :

$$
\left\{\exp \left(t \hat{\varphi}^{*}\left(2 i \pi \alpha z \frac{\partial}{\partial z}\right)\right) ; t \in \mathbb{C}\right\}
$$

où $a=e^{2 i \pi \alpha}$. Les éléments sont des difféomorphismes a priori formels qui convergent au moins pour les valeurs entières de $t$. Ce groupe à 1 paramètre est unique, toujours d'après le Corollaire 2.18, au choix près de la détermination $\alpha$ du logarithme de a (le groupe est le même mais sa paramétrisation change). Si $f$ n'est pas analytiquement linéarisable, alors 
l'ensemble des $t \in \mathbb{C}$ pour lesquels $\hat{\varphi}^{*}\left(z \mapsto e^{2 i \pi \alpha t} z\right)$ converge (et appartient à $\operatorname{Diff}(\mathbb{C}, 0))$ doit satisfaire e $e^{2 i \pi t \alpha} \in \mathbb{S}^{1} \backslash \mathcal{B}$ où $\mathbb{S}^{1}$ est le cercle unité. Le complémentaire de $\mathcal{B}$ dans $\mathbb{S}^{1}$ est de mesure nulle mais non dénombrable.

Notamment, R. Pérez-Marco démontre dans [169] le :

Théorème 3.11 (Pérez-Marco). Il existe des sous-groupes abéliens non dénombrables de $\operatorname{Diff}(\mathbb{C}, 0)$ qui sont formellement linéarisables mais non analytiquement linéarisables.

Remarque 3.12. Nous devons à S. Lamy l'exemple suivant (voir [104]). Considérons un groupe linéaire $G_{0}$ infiniment engendré par des rotations périodiques :

$$
G_{0}=<f_{1}, f_{2}, f_{3}, \ldots>, \quad f_{n}(z)=e^{2 i \pi \frac{p_{n}}{q_{n}} z}
$$

d'ordre $q_{n}$ arbitrairement grand. Quitte à remplacer le $n^{\text {ème }}$ générateur $f_{n}$ par un générateur du sous-groupe engendré par $\left\langle f_{1}, f_{2}, f_{3}, \ldots, f_{n}\right\rangle$, on peut supposer que $q_{n}$ divise $q_{n+1}$ tout $n \in \mathbb{N}^{*}$. On construit un groupe $G$ formellement conjugué à $G_{0}$, mais non analytiquement conjugué de la manière suivante. Considérons le difféomorphisme formel

$$
\hat{\varphi}(z)=z+\sum_{n}\left(q_{n} !\right) z^{q_{n}+1} \in \widehat{\operatorname{Diff}}(\mathbb{C}, 0) .
$$

Visiblement, $\hat{\varphi}$ diverge. Par contre, $G=\hat{\varphi}^{*} G_{0}$ est bien un sous-groupe de $\operatorname{Diff}(\mathbb{C}, 0)$. En effet, seul le jet d'ordre $q_{n}$ affecte $f_{n}$ et par suite $\hat{\varphi}^{*} f_{n}$ est convergent. Cependant, toute autre conjugaison formelle de $G$ à $G_{0}$ s'écrit $\psi \circ \hat{\varphi}$ avec $\psi \in \widehat{\operatorname{Diff}}(\mathbb{C}, 0)$ commutant $\grave{a} G_{0} ;$ mais ceci implique que $\psi$ est linéaires et $\psi \circ \hat{\varphi}$ diverge.

\subsection{Les difféomorphismes tangents à l'iden- tité : survol historique}

L'étude des difféomorphismes tangents à l'identité a débuté il y a plus d'un siècle avec l'article de Léopold Leau et n'a cessé de se développer depuis. Les articles de P. Fatou, G.D. Birkhoff, J. Hadamard, G. Szekeres, P. Erdös, E. Jabotinsky, I.N. Baker, T. Kimura, J. Écalle, C. Camacho, S.M. Voronin, B. Malgrange, J. Martinet, J.-P. Ramis, I. Nakai, J. Rey, J.-M. Trépreau, P. Ahern, J.-P. Rosay, X. Gong, G. Casale, D. Sauzin, F. Fauvet, M. Resman témoignent de l'importance du sujet. Nous verrons dans la suite que ces difféomorphismes jouent un rôle central dans la théorie des sous-groupes de Diff( $(\mathbb{C}, 0)$ et nous allons leur consacrer la principale partie de ce chapitre. Nous nous livrons ici à un petit historique. 
En 1897, Léopold Leau publie dans [106] la première étude consacrée à la résolution de l'équation d'Abel (voir [2]) :

$$
\psi \circ f=\psi+1
$$

où $f(z)=z+z^{p+1}+\cdots$, et $\psi$ est l'inconnue. Il commence par construire une collection de $2 p$ ouverts sectoriels, disposés autour de l'origine comme les pétales d'une fleur, sur lesquels $f$ est tantôt contractante, tantôt dilatante. C'est le théorème de la fleur. Ensuite, Leau construit sur chaque pétale une solution $\psi$ à l'équation d'Abel, conjugant ainsi la dynamique de $f$ avec celle de la translation $w \mapsto w+1$. C'est la première version du lemme de normalisation sectorielle. On comprend bien la démarche de Leau si l'on se place dans la coordonnée $w=-\frac{1}{p z^{p}}$ : la dynamique de $f$ est proche de la translation $w \mapsto w+1$ au voisinage de l'infini et l'est d'autant plus que l'on s'approche de l'infini. Les pétales invariants par $f$ et $f^{\circ}(-1)$ apparaissent clairement. Ensuite Leau construit une forme différentielle holomorphe invariante par $f$, par exemple sur un pétale attractif, en allant chercher la forme $d w$ à l'infini, invariante par la translation, et en la ramenant par $f$ : la famille de formes $\left(f^{\circ n}\right)^{*} d w=d f^{\circ n}$ converge uniformément sur le pétale vers une forme $\omega$ invariante par $f$. En intégrant l'identité $f^{*} \omega=\omega$, il vient $\psi \circ f=\psi+$ constante où $\psi:=\int_{w_{0}}^{w} \omega$; par construction, $\psi$ est tangente à une translation à l'infini et la constante d'intégration doit être 1 . Finalement, on récupère, sur chaque pétale, une solution $\psi$ à l'équation d'Abel de la forme $\psi(z)=-\frac{1}{p z^{p}}+\cdots$.

En 1919, Pierre Fatou redémontre ce résultat dans [77] en suivant la même démarche que celle que nous avons exposée dans la preuve du théorème de Kønigs : il s'agit, dans la variable $w$, de construire $\psi$ comme limite de $f^{\circ n}-n$. Il commence par réduire $f$ sous la forme $w \mapsto w+1+\frac{\lambda}{2 i \pi p} \frac{1}{w}+\cdots$, où $\lambda$ est précisément l'invariant formel défini dans la Proposition 2.16. Lorsque $\lambda=0, f^{\circ n}-n$ converge uniformément vers une solution $\psi$ à l'équation d'Abel. Hélas, dès que $\lambda \neq 0$, on voit apparaitre dans $f^{\circ n}-n$ un terme de l'ordre de $\frac{\lambda}{2 i \pi p} \log (n)$ qui fait diverger le procédé. Fatou s'en sort en montrant que $f^{\circ n}-n-\frac{\lambda}{2 i \pi p} \log (n)$ converge et la limite $\psi$ est encore solution de l'équation d'Abel.

Ensuite, suivront de nombreuses variantes, notamment de G.D. Birkhoff, G. Szekeres, T. Kimura, J. Écalle, S.M. Voronin, B. Malgrange, J. Martinet et J.-P. Ramis, Y. Ilyashenko et I. Nakai. En 1975, Jean Écalle apporte un nouveau point de vue. Si $f=\exp \left(X_{p, \lambda}\right)$, une solution à l'équation d'Abel est donnée par $\psi_{p, \lambda}(z)=-\frac{1}{p z^{p}}+\frac{\lambda}{2 i \pi} \log (z)$. Dans le cas général, la solution $\psi$ se décompose naturellement sous la forme $\psi=\psi_{p, \lambda} \circ \varphi$, ce qui nous donne une conjugaison sectorielle $\varphi$ entre $f$ et son modèle formel $\exp \left(X_{p, \lambda}\right)$. Il montre alors que $\varphi$ admet la coordonnée formelle $\hat{\varphi}$ comme développement asymptotique à l'origine. Des développements asymptotiques apparaissent déjà chez Birkhoff, Szekeres et Kimura mais plutôt pour la solution $\psi$. 
C'est là la seule amélioration substentielle du résultat de Leau apportée par les multiples démonstrations.

Le corollaire essentiel de la trivialisation sectorielle que nous allons utiliser à tours de bras est que l'image de $\psi$ contient un domaine fondamental pour $w \mapsto w+1$, c'est-à-dire que le quotient de chaque pétale par $f$ est un cylindre isomorphe à $\mathbb{C}^{*}$. Ce fait se déduit déjà de l'article de Leau.

En 1939, George D. Birkhoff donne dans [17] les premiers exemples de difféomorphismes qui ne sont pas plongeables dans un groupe à 1 paramètre, i.e. pour lesquels la coordonnée formelle normalisante $\hat{\varphi}$ construite dans la Proposition 2.16 diverge; il refait la construction de Leau et de Fatou et en déduit une liste complète d'invariants analytiques pour les difféomorphismes résonants. La seconde partie de son article pose le problème inverse, à savoir comment réaliser tous ces invariants par des dynamiques résonantes : l'auteur conjecture que oui après avoir construit une déformation à un paramètre de germes tangents à l'identité dont les invariants varient continûment. Dans la dernière partie, Birkhoff donne des conditions nécessaires et suffisantes à l'existence de fonctions méromorphes multiformes invariantes par un sous-groupe de Diff( $\mathbb{C}, 0)$; il donne l'écriture générale de ces intégrales premières dans certains cas. Ce mémoire très complet n'a pas attiré l'attention à l'époque et est tout de suite tombé dans l'oubli ; Jean-Pierre Ramis a sorti ce travail de l'ombre il y a quelques années de cela et il est surprenant de voir combien de décennies ont été nécessaires pour que tous ces résultats aient de nouveau été redémontrés. Notamment, le problème des intégrales premières est toujours d'actualité. Oublions le travail de Birkhoff et regardons comment se sont développées les différentes idées.

Le problème de l'itération, tel qu'il était posé dans [101], [107] ou [216], consistait, étant donnée une transformation $f$ réelle ou complexe, d'une ou plusieurs variables, à construire un groupe à un paramètre $(t, z) \mapsto$ $f^{\circ t}(z)$ décrivant explicitement pour $t \in \mathbb{Z}$ les itérées successives de $f$; dans un second temps, on cherchait à étendre cette flêche aux temps $t$ non entiers. Dès la fin du siècle dernier, il était clair que ce problème était intimement lié à la résolution de l'équation d'Abel (voir [2]). En effet, toute solution $\psi$ nous fournit le groupe à un paramètre $f^{\circ t}(z)=\psi\left(\psi^{\circ}(-1)(z)+t\right)$. Réciproquement, Paul Lévy remarque dans [107] qu'un point $z_{0}$ étant fixé, on récupère $\psi$ en inversant la flêche $t \mapsto f^{\circ t}\left(z_{0}\right)$.

Le théorème de Leau nous dit que tout difféomorphisme tangent à l'identité est pleinement itérable sur chaque pétale; la question restait ouverte au voisinage de l'origine. Ce problème a été considéré du point de vue formel par Eri Jabotinsky vers 1942 dont le travail est relaté par Jacques Hadamard (voir [89]) : il montre que $f^{\circ t}(z) \in \mathbb{C}[t][[z]]$. Ce groupe à 1 paramètre est donné par $\hat{\varphi}^{*} \exp \left(t \cdot X_{p, \lambda}\right)$ si l'on reprend les notations de la Proposition 2.16. Hadamard rapproche ce travail de celui de Luntz qui cherchait à décrire les difféomorphismes formels tangents à l'identité com- 
mutant avec $f$ et remarque que ces problèmes sont les mêmes. I.N. Baker reprend ces idées de façon systématique en 1962 (voir [6]) et montre que l'ensemble des valeurs de $t$ pour lesquelles la série $f^{\circ t}(z)$ converge est discret ou plein dans $\mathbb{C}$. Il avait lui-même donné un exemple explicite de germe non pleinement itérable deux ans plus tôt : il montrait que seules les itérées entières de $z \mapsto e^{z}-1$ convergeaient. La question de l'existence de germes pour lesquels l'ensemble d'itération effective est un réseau était posée et restera ouverte jusqu'en 1973 où J. Écalle et L.S.O. Liverpool montrent indépendament (voir $[70,113]$ ) que cela n'arrive pas. Simultanément au travail de Baker, Erdös et Jabotinsky construisent dans [75] le générateur infinitésimal formel $\hat{X}$ du flot $f^{\circ t}(z)$ et montrent que $f$ est pleinement itérable si, et seulement si, $\hat{X}$ converge. Avec nos notations, $\hat{X}$ est le champ de vecteurs formel défini par $\varphi^{*} X_{p, \lambda}$. En 1963, Jabotinsky donne les formules combinatoires liant les coefficients de $f$ à ceux de $\hat{X}$ et, en 1966, il borne la divergence de $\hat{X}$ (voir $[95,96]$ ). Pendant ce temps, les exemples de germes non pleinement itérable se multiplient. En 1964, Szekeres montre dans [217] que les germes donnés par des fonctions rationnelles ou entières ne sont pas pleinement itérables (excepté $z \mapsto z /(1+t z)$ ); la même année, Baker étend ce résultat aux fontions méromorphes dans [7]. Plus généralement, lorsque $\lambda \neq 0$, un germe de difféomorphisme admettant un prolongement analytique en dehors d'un ensemble dénombrable sur $\mathbb{C}$ avec un nombre fini de déterminations ne peut être pleinement itérable : Baker le démontrera dans le cas $p=1$ dans [8], puis Écalle en toute généralité dans [70]. Les modèles formels $a \exp \left(X_{p, \lambda}\right)$ sont décrits (du point de vue prolongement analytique et dynamique) dans [4] et ne sont pas algébriques pour $\lambda \neq 0$.

Si une théorie formelle s'est développée au cours des travaux précédemment cités, à aucun moment il n'est explicitement question de classification formelle des difféomorphismes. Celle-ci n'est en fait apparue qu'en 1971 (si l'on oublie que c'était fait chez Birkhoff!) dans le travail de Kimura (voir [98]) et, sous la forme définitive exposée ici, dans l'article de Jean Écalle de 1975. Ce dernier montre qu'un difféomorphisme est pleinement itérable si et seulement si $\hat{\varphi}$ converge et le lien est fait avec ce qui précède.

La classification analytique des difféomorphismes résonants consiste à exhiber une liste exhaustive et non redondante d'invariants holomorphes. La liste complète de ces invariants a été trouvée par G.Birkhoff en 1939 puis oubliée et retrouvée par J. Écalle en 1973. La réalisation de tous ces invariants a été faite indépendamment par J. Écalle, B. Malgrange et S.M. Voronin en 1981 (voir [71, 126, 225]).

La présentation de ces invariants diffère selon la littérature choisie; la plus classique consiste à interpréter les coordonnées sectorielles $\psi$ comme un atlas trivialisant la dynamique et à considérer les changements de cartes; cette idée était déjà suggérée chez Leau et c'est essentiellement celle de Birkhoff, Écalle (dans son article de 1975), Malgrange et Voronin 
à quelques nuances près; par exemple, chez Birkhoff, ces changements de cartes sont vus à travers les intégrales premières de la dynamique triviale (celle de la forme normale formelle), point de vue que l'on retrouve chez Martinet et Ramis, alors que chez Voronin, ils sont vus à travers leurs coefficients de Fourier; par contre, la présentation ultérieure d'Écalle via les fonctions résurgentes en 1981 (voir [71]) tranche radicalement avec les précédentes, et il n'est pas facile de faire le lien entre les coefficients de résurgence d'Écalle et les coefficients de Fourier de Voronin (voir [67, 68]).

En 1983, J. Martinet et J.-P. Ramis réécrivent cette classification en termes de chapelets de sphères dans [139]. C'est cette présentation que nous allons adopter : elle est, à nos yeux, la plus géométrique et la plus adatée à nos problèmes.

Le corollaire fondamental du théorème de Leau est que le quotient de chaque pétale par $f$ est un cylindre infini, isomorphe à la sphère de Riemann épointée $\mathbb{C}^{*}=\overline{\mathbb{C}} \backslash\{0, \infty\}$. On obtient alors le quotient d'un voisinage épointé de l'origine $U^{*}:=U \backslash\{0\}$ par $f$ en effectuant sur ces $2 \nu$ sphères les identifications imposées par les chevauchements des pétales. La structure analytique de ce quotient, baptisé chapelet de sphères par J. Martinet et J.-P. Ramis, ne dépend que de la classe analytique de $f$. La classification analytique des difféomorphismes tangents à l'identité nous dit que la flêche $f \mapsto U^{*} / f$ induit une bijection entre l'ensemble des classes analytiques de difféomorphismes $f(z)=z+z^{p+1}+\cdots$ et l'ensemble des chapelets de $2 p$ sphères.

De cette construction, on déduit aisément le Théorème d'Écalle-Liverpool : les difféomorphismes commutant à $f$ correspondent géométriquement à des automorphismes du chapelet $U^{*} / f$. On vérifie alors facilement qu'ils sont en nombre fini dès que $f$ n'est pas pleinement itérable. Je dois cette idée de démonstration à E. Paul. De la même manière, on déduit la classification des germes de difféomorphismes analytiques réels en les considérant comme des germes complexes commutant à une involution anti-holomorphe : celleci induit une involution anti-holomorphe sur le quotient $U^{*} / f$ apportant des restrictions explicites sur la structure analytique du chapelet. Dans le même ordre d'idées, Nakai considère dans [152] la classification des paires de courbes analytiques réelles tangentes à l'origine de $\mathbb{C}:$ en composant leur réflexion de Schwarz, on obtient un germe de difféomorphisme tangent à l'identité $f$ qui anti-commute à chacune des réflexions. La classification de ces objets se ramène de nouveau à celle des chapelets munis d'une involution anti-holomorphe. Jean-Marie Trépreau complète cette approche dans [221] en construisant des paires d'ellipses tangentes donnant naissance à de nouveaux difféomorphismes explicites non pleinement itérables. Dans leur récent survey [3], P. Ahern et X. Gong reprennent en détails tous ces problèmes de symétries; nous nous contenterons ici d'en tracer les principales idées.

L'existence de développements asymptotiques pour les coordonnées de 
Fatou a été établie par Birkhoff, Szekeres, Kimura et Écalle. Une étude plus fine de l'asymptoticité que l'on peut trouver chez Écalle ou Martinet et Ramis montre qu'elle est Gevrey d'ordre $p$ et que $\hat{\varphi}$ est $p$-sommable. Mieux, dans ce cas la transformée de Borel de $\hat{\varphi}$ converge et J. Écalle montre en 1981 qu'elle admet un prolongement analytique sans fin sur $\mathbb{C} \backslash 2 i \pi \mathbb{Z}^{*}$ avec de très bonne propriétés de croissance à l'infini : c'étaient les premiers exemples de fonctions résurgentes. La p-sommabilité de la transformation normalisante $\hat{\varphi}$ ne sera utilisée que pour démontrer le Théorème de Nakai sur la dynamique des sous-groupes non résolubles de Diff(C, 0$)$ (voir [151]) et le Théorème de rigidité de Cerveau et Moussu (voir [58]).

La classification topologique des difféomorphismes tangents à l'identité a été faite par César Camacho en 1978 (voir [27]) : le nombre $p$ de pétales est le seul invariant topologique, ce qui nous donne une version forte du théorème de la fleur. On peut citer au passage la coïncidence entre classifications analytique et $C^{1}$-réelle, que l'on trouvera dans l'exposé de Martinet et Ramis dont nous nous inspirerons beaucoup, et l'optimalité de ce résultat, démontrée par Jérome Rey dans sa thèse [186] : deux difféomorphismes $z+z^{p+1}+\cdots$ sont toujours conjugués par une transformation différentiable à l'origine (mais pas $C^{1}$ !) et $C^{\infty}$ ailleurs. Plus récemment, les propriétés métriques de la dynamiques ont été étudiées par Maja Resman dans [185].

\subsection{Le théorème de la fleur}

Dans la suite, $f$ désigne un difféomorphisme tangent à l'identité :

$$
f(z)=z+z^{p+1}+\cdots \in \operatorname{Diff}(\mathbb{C}, 0)
$$

(on peut supposer le coefficient de $z^{p+1}$ unitaire quitte à conjuguer $f$ par une homothétie) et $U$ un disque ouvert sur lequel $f$ et $f^{\circ(-1)}$ sont bien définis et injectifs. La première description de la dynamique induite par itération positive et négative de $f$ au voisinage $U$ de 0 remonte à la thèse de Leau dans laquelle on trouve la toute première version du Théorème de la Fleur :

Théorème 3.13 (Leau). Quitte à rétrécir $U$, il existe un recouvrement de $U \backslash\{0\}$ par $2 p$ ouverts sectoriels, disposés autour de l'origine comme les pétales d'une fleur autour de son cœur, sur lesquels la dynamique de $f$ est tantôt contractante, tantôt dilatante :

Numérotés par $k=0, \ldots, p-1$, les p pétales attractifs $V_{k}^{+}$sont coïncés dans les $p$ composantes connexes de $\left\{z \in U ; z^{p} \notin \mathbb{R}^{+}\right\}$, les $p$ pétales répulsifs $V_{k}^{-}$, dans les $p$ composantes connexes de $\left\{z \in U ; z^{p} \notin \mathbb{R}^{-}\right\}$et 


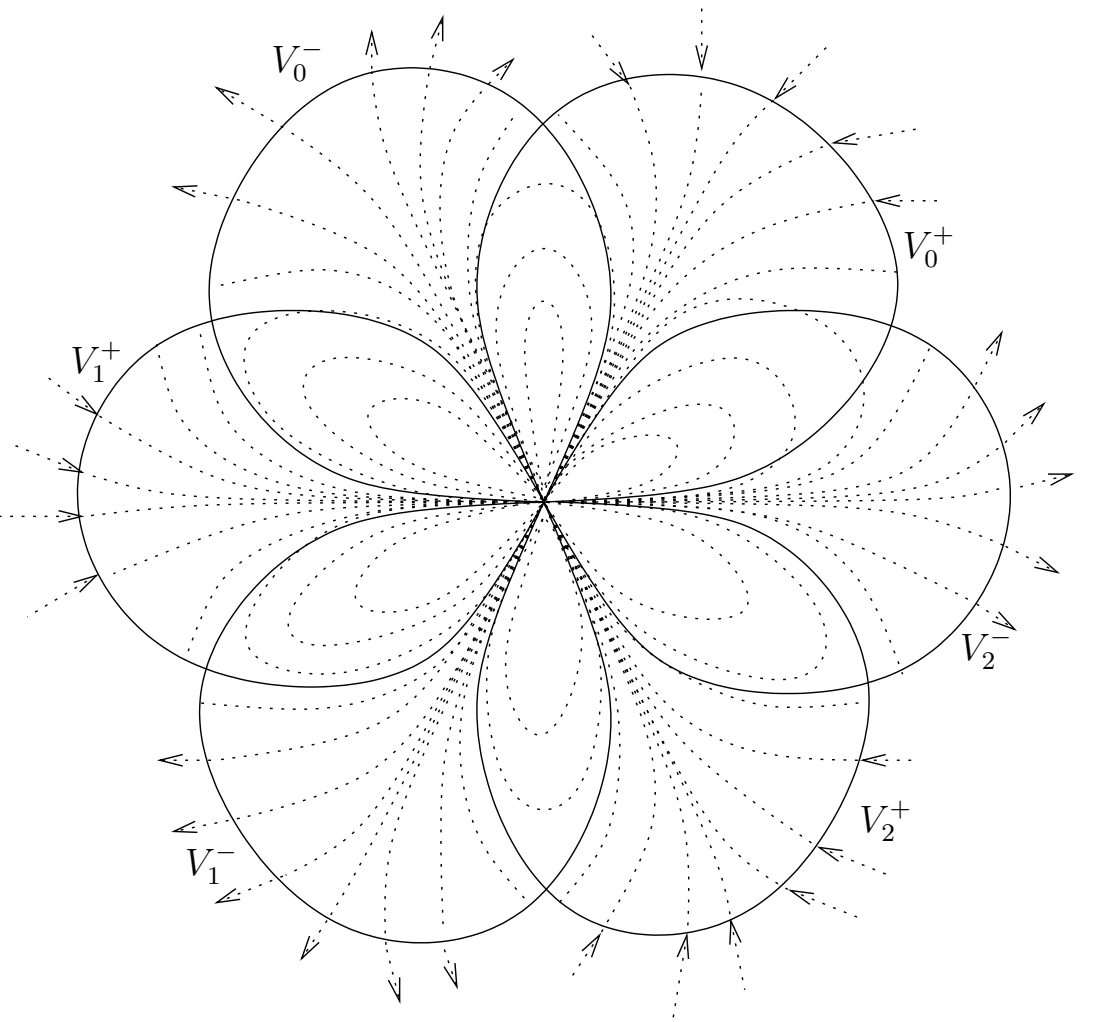

Figure 3.2 - La fleur et ses pétales 
pour $k=0, \ldots, p-1$ on $a:$

$$
\begin{aligned}
f\left(V_{k}^{+}\right) & \subset V_{k}^{+} \quad \text { et } \quad \lim _{n \rightarrow+\infty}\left\|f^{\circ n}\right\|_{V_{k}^{+}}=0, \\
f^{\circ(-1)}\left(V_{k}^{-}\right) & \subset V_{k}^{-} \quad \text { et } \quad \lim _{n \rightarrow+\infty}\left\|f^{\circ(-n)}\right\|_{V_{k}^{-}}=0 .
\end{aligned}
$$

Plus précisément, sur chaque pétale, on a une estimation du type:

$$
\left|f^{\circ n}(z)\right|<C|z| /\left(1+n|z|^{p}\right)^{1 / p} \quad \text { pour une constante } C>0 .
$$

Nous appellerons ces ouverts pétales attractifs et répulsifs de la dynamique.

Démonstration. Dans la variable $w:=-\frac{1}{p z^{p}}$, chacune des $p$ déterminations de la dynamique est de la forme :

$$
\tilde{f}(w)=w+1+\epsilon(w)
$$

où $|\epsilon(w)|$ est un $o\left(\frac{1}{|w|}\right)$ à l'infini. On peut supposer que $|\tilde{f}(w)-w-1|<$ $\varepsilon<<1$ uniformément sur notre ouvert. La figure suivante nous donne l'allure d'un pétale attractif $\tilde{V}^{+}$dans la variable $w$, d'ouverture $2 \pi-2 \theta$, pour un $\varepsilon<\theta<\frac{\pi}{2}$.

Maintenant, un calcul un peu plus fin nous dit qu'il existe une constante $C>0$ telle que :

$$
\left|\tilde{f}^{\circ n}(w)\right|>C(|w|+n) \quad \text { sur } \quad \tilde{V}^{+} .
$$

Il suffit pour cela de minimiser l'expression $\frac{\left|\tilde{f}^{0 n}(w)\right|}{(|w|+n)}$ en fonction des trois variables réelles $\arg (w), n$ et $|w|$; à $n$ et $|w|$ fixés, le minimum est atteint pour $w \in \partial \tilde{V}^{+}$, i.e. $\arg (w) \sim \pm(\pi-\theta)$; maintenant, $w \in \partial \tilde{V}^{+}$étant fixé, le minimum est de nouveau atteint pour $n \sim|w| \cos (\theta)$ et est équivalent à $\frac{\tan (\theta)-\varepsilon}{1+\cos (\theta)} \cos (\theta)$.

\subsection{Trivialisations sectorielles}

On cherche ici à conjuguer $f$ à la translation $w \mapsto w+1$ sur chaque pétale, c'est-à-dire à construire des solutions sectorielles à l'équation d'Abel :

$$
\psi \circ f=\psi+1
$$

Lorsque $f=\exp \left(X_{p, \lambda}\right)$ (voir Remarque 2.4), la solution s'obtient en intégrant la forme associée :

$$
\omega_{p, \lambda}=\frac{d z}{z^{p+1}}+\frac{\lambda}{2 i \pi} \cdot \frac{d z}{z}
$$




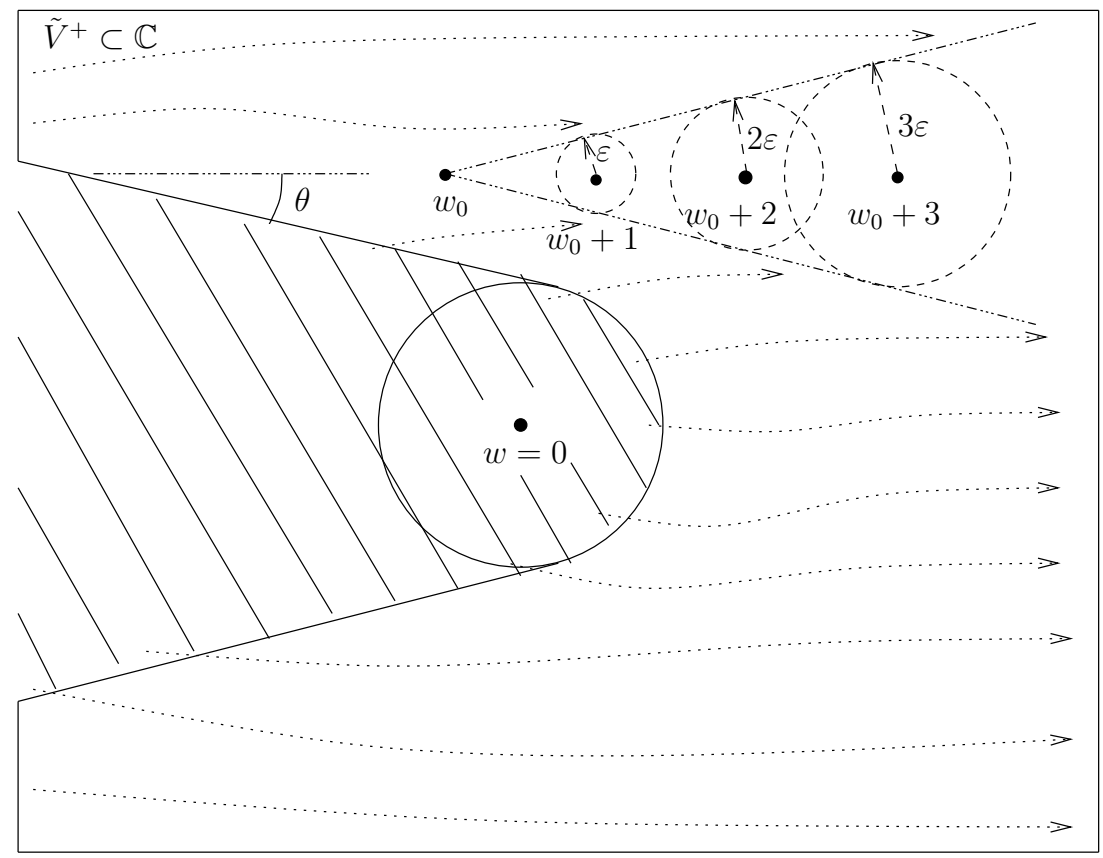

FIgURE 3.3 - Itération dans la variable $w$

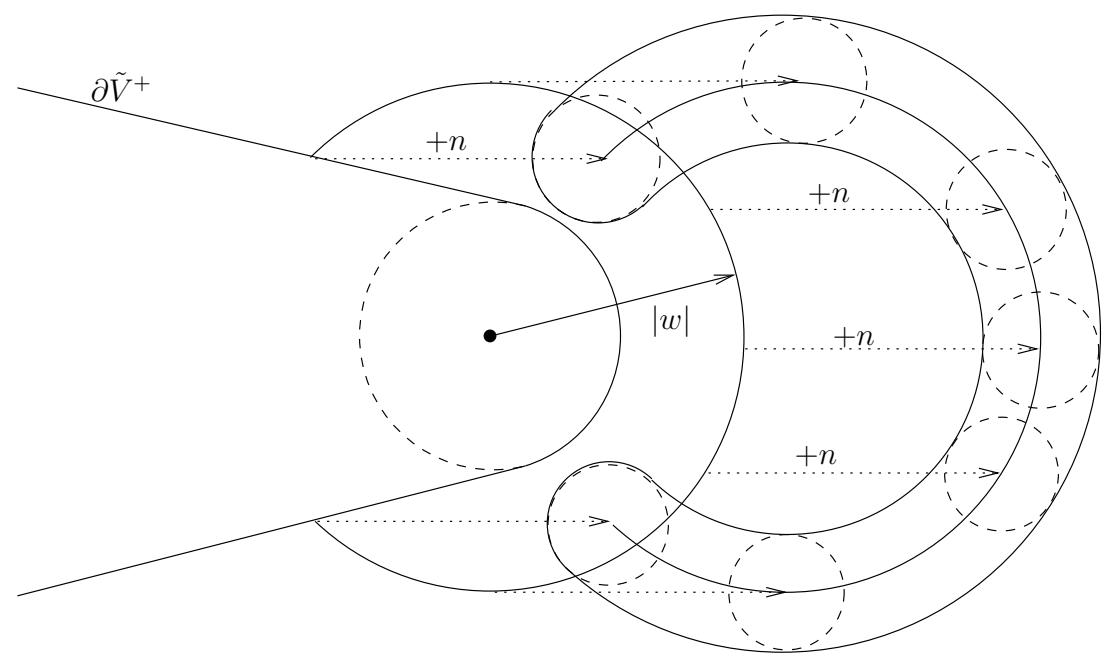

Figure $3.4-$ Minimiser $\frac{\left|\tilde{f}^{\circ n}(w)\right|}{(|w|+n)}$ 
de façon à redresser le champ $X_{p, \lambda}$, ce qui nous donne :

$$
\psi_{p, \lambda}(z)=-\frac{1}{p z^{p}}+\frac{\lambda}{2 i \pi} \log (z) .
$$

On obtient dans ce cas une solution "globale" à la constante de monodromie près :

$$
\psi_{p, \lambda}\left(e^{2 i \pi} z\right)=\psi_{p, \lambda}(z)+\lambda
$$

Dans le cas général, ce problème a été aussi résolu par Leau en 1897.

Un point de vue plus récent consiste à conjuguer $f$ à son modèle formel $\exp \left(X_{p, \lambda}\right)$ par une transformation $\varphi$, c'est-à-dire :

$$
\varphi \circ f=\exp \left(X_{p, \lambda}\right) \circ \varphi \text {. }
$$

Les deux approches sont équivalentes puisque :

$$
\psi=\psi_{p, \lambda} \circ \varphi
$$

Sous ce point de vue, on peut demander en outre que $\varphi$ admette la solution formelle $\hat{\varphi}$ construite dans la Proposition 2.16 comme développement asymptotique à l'origine : si $\hat{\varphi}=\sum_{n>0} u_{n} z^{n}$, alors ceci signifie que $\varphi(z)-\sum_{n=1}^{N} u_{n} z^{n}$ est un $o\left(|z|^{N}\right)$ pour tout $N \in \mathbb{N}$. Ceci nous donne pour $\psi$ un développement asymptotique du type :

$$
\hat{\psi}=\psi_{p, \lambda} \circ \hat{\varphi}=\frac{\lambda}{2 i \pi} \log (z)+\sum_{n \geq-p} u_{n} z^{n}
$$

C'est sous cette dernière forme que le développement asymptotique des trivialisations sectorielles est apparu dans les travaux de Birkhoff, Szekeres et Kimura et c'est dans l'article d'Écalle de 1975 que le lien est fait entre les différentes approches, comme nous venons de le décrire.

A présent, construisons, par l'algorithme de la Proposition 2.16, une coordonnée dans laquelle :

$$
f(z)=\exp \left(X_{p, \lambda}\right)+o\left(|z|^{2 p+1}\right)=z+z^{p+1}+\left(\frac{p+1}{2}-\frac{\lambda}{2 i \pi}\right) z^{2 p+1}+\cdots,
$$

de sorte que $\hat{\varphi}(z)=z$ modulo $z^{2 p+2}$ et supposons donné un recouvrement de Leau pour $f$.

Théorème 3.14 (Leau + Ecalle). Il existe, sur chaque pétale $V$, un plongement $\psi: V \hookrightarrow \mathbb{C}$ unique à constante additive près conjugant la dynamique de $f$ à celle de la translation $w \mapsto w+1$ :

$$
\psi \circ f=\psi+1
$$


Le comportement de $\psi$ à l'origine est donné par :

$$
\psi(z)=\psi_{p, \lambda}(z)+\varepsilon(z) \quad \text { avec } \quad \lim _{z \rightarrow 0} \varepsilon(z)=\text { constante. }
$$

Si l'on note $\varphi$ la normalisante sectorielle définie par $\psi=\psi_{p, \lambda} \circ \varphi$, en choisissant $\lim _{z \rightarrow 0} \varepsilon(z)=0$, alors $\varphi$ admet $\hat{\varphi}$ comme développement asymptotique à l'origine.

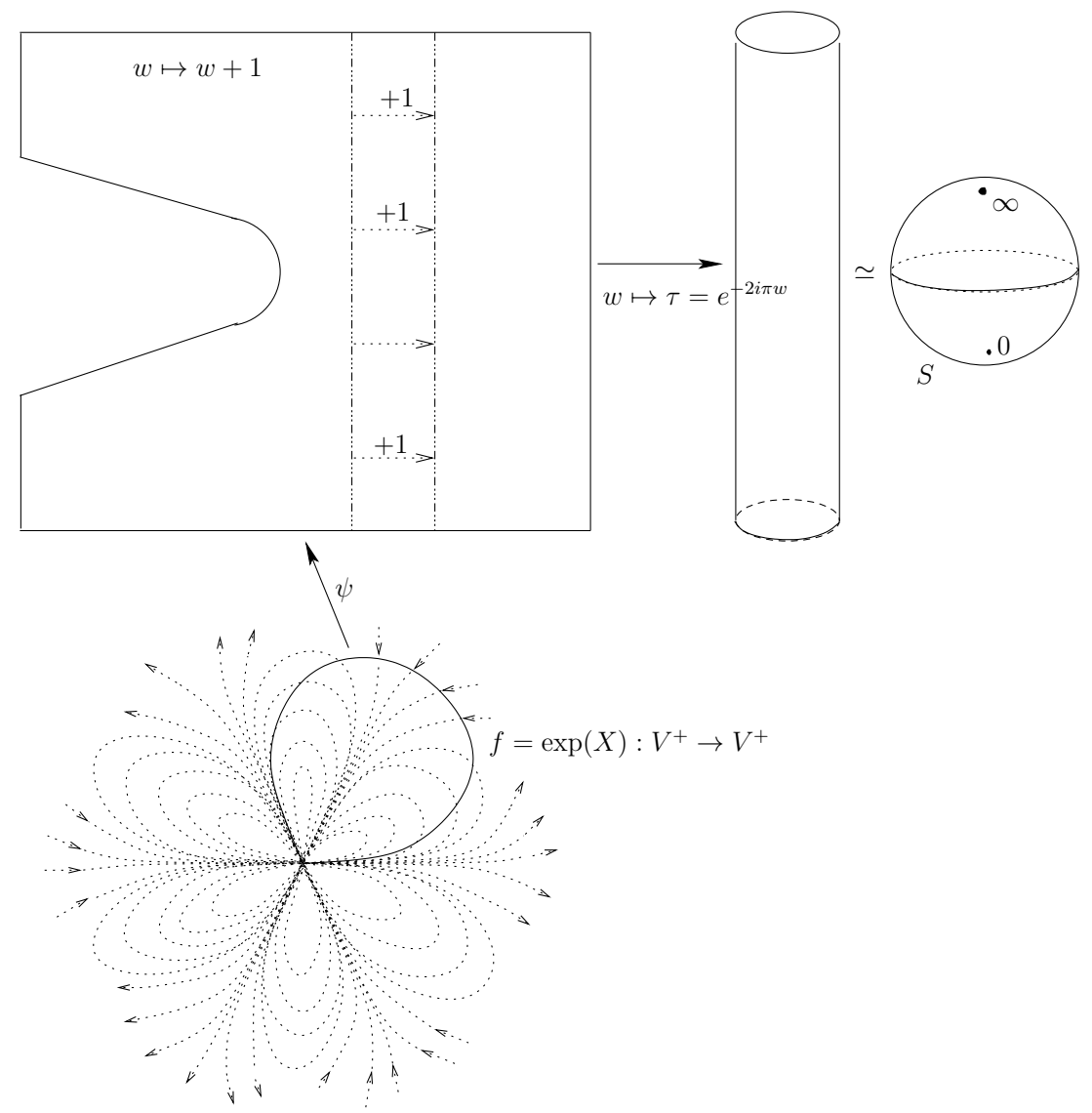

FiguRE 3.5 - Trivialisation sectorielle : coordonnée de Leau-Fatou

On notera $w$ la coordonnée sectorielle donnée par $w=\psi(z)$. Sur chaque pétale $V, f$ est le flot au temps 1 d'un unique champ de vecteurs holomorphe $X$ :

$$
f=\exp (X) \quad \text { où } \quad X=\psi^{*} \frac{\partial}{\partial w} \text {, }
$$


et l'itérée complexe $f^{\circ t}, t \in \mathbb{C}$, y est bien définie :

$$
f^{\circ t}=\exp (t X) \quad \text { et } \quad \psi \circ f^{\circ t}=\psi+t .
$$

Par construction, $X$ admet le champ de vecteur formel $\hat{\varphi}^{*} X_{p, \lambda}$ comme développement asymptotique à l'origine. Chez Écalle, $X$ est le logarithme itératif sectoriel de $f$.

Le passage au quotient d'un pétale $V$ par la dynamique est donné par l'intégrale première :

$$
H: V \rightarrow S \simeq \overline{\mathbb{C}} \backslash\{0, \infty\} ; z \mapsto \exp (-2 i \pi \psi(z)) .
$$

La présence du signe - dans l'intégrale première n'est pas très naturelle; la raison de ce choix apparaitra plus loin, lorsque nous parlerons du nœudcol. Dans la coordonnée $\tau=H(z)$ du quotient $S$, la dynamique des itérées complexes $f^{\circ t}, t \in \mathbb{C}$ devient linéaire :

$$
H_{*} f^{\circ t}: \tau \mapsto e^{-2 i \pi t} \tau \quad \text { et } \quad H_{*} X=-2 i \pi \tau \frac{\partial}{\partial \tau} .
$$

Ces quotients sont parfois appelés cylindres d'Écalle.

Démonstration. Nous allons chercher la transformation $\psi$ sous la forme :

$$
\psi=\psi_{p, \lambda}+\phi .
$$

En substituant à l'équation d'Abel, il vient :

$$
\phi \circ f-\phi=\Delta
$$

où $\Delta:=\psi_{p, \lambda}+1-\psi_{p, \lambda} \circ f$. Si $\left|f-\exp \left(X_{p, \lambda}\right)\right|$ est un $O\left(\left|z^{2 p+k+1}\right|\right), k \in \mathbb{N}^{*}$, alors $|\Delta|$ est un $O\left(\left|z^{p+k}\right|\right)$ et la somme :

$$
-\sum_{n \geq 0} \Delta \circ f^{\circ n} \quad\left(\operatorname{resp} . \sum_{n<0} \Delta \circ f^{\circ n}\right)
$$

converge uniformément sur chaque pétale attractif (resp. répulsif) vers une solution $\phi$ de cette dernière équation. En effet, dans la variable $w=-\frac{1}{p z^{p}}$, $|\tilde{\Delta}|$ est un $O\left(\left|\frac{1}{w^{1+k / p}}\right|\right)$ et donc :

$$
\left|\tilde{\Delta} \circ \tilde{f}^{\circ n}(w)\right|<\frac{C}{(|w|+n)^{1+k / p}}
$$

pour une constante $C>0$ (estimation de la preuve précédente); en sommant, on obtient de surcroît que $|\tilde{\phi}|$ est un $O\left(\frac{1}{|w|^{k / p}}\right)$, i.e. que $|\phi|$ est un $O\left(|z|^{k}\right)$. 
La transformation $\psi:=\psi_{p, \lambda}+\phi$ est construite comme limite uniforme, par exemple sur un pétale attractif, des transformations :

$\psi_{N}:=\psi_{p, \lambda}-\sum_{n=0}^{N} \Delta \circ f^{\circ n}=\psi_{p, \lambda} \circ f^{\circ N}-N=\psi_{p, \lambda}\left(\exp \left(-N \cdot X_{p, \lambda}\right) \circ f^{\circ N}\right)$

L'injectivité de $\psi_{N}$ résulte de l'injectivité sectorielle de $\psi_{p, \lambda}$, et celle de $\psi$, du théorème de Rouché-Hurwitz. La transformation intermédiaire $\varphi$ est alors obtenue par convergence uniforme :

$$
\varphi:=\lim _{N \rightarrow+\infty} \exp \left(-N X_{p, \lambda}\right) \circ f^{\circ N}
$$

et envoie par construction la dynamique de $f$ sur celle de son modèle formel $\exp \left(X_{p, \lambda}\right)$. Nos estimations montrent que si $\left|f-\exp \left(X_{p, \lambda}\right)\right|$ est un $O\left(|z|^{2 p+k+1}\right)$, alors $|\varphi(z)-z|$ est un $O\left(|z|^{p+k+1}\right)$, c'est-à-dire $\varphi$ a le même ordre de contact à l'identité que la coordonnée formelle $\hat{\varphi}$. On en déduit que $\varphi$ admet $\hat{\varphi}$ comme développement asymptotique à l'origine.

Remarque 3.15. Dans la variable $w$, la solution $\psi$ est construite comme limite de $\tilde{f}^{\circ n}-n-\frac{\lambda}{2 i \pi p} \log \left(f^{\circ n}\right)$ ce qui est un peu plus compliqué que la démarche de Fatou; cependant, dans la variable $z$, la solution $\varphi$ est obtenue comme limite des transformations $f_{0}^{\circ(-n)} \circ f^{\circ n}$ où $f_{0}$ est le modèle formel $\exp \left(X_{p, \lambda}\right)$ et nous retrouvons la simplicité de la démonstration de Kœnigs.

\subsection{La cohomologie des trivialisation secto- rielles}

Le système de coordonnées euclidiennes donné par la collection

$$
\left(w_{k}^{+}:=\psi_{k}^{+}(z), w_{k}^{-}:=\psi_{k}^{-}(z)\right)_{k \in \mathbb{Z} / p \mathbb{Z}}
$$

des trivialisantes sectorielles définit un atlas trivialisant la dynamique de $f$ dont les changements de cartes sont donnés par les applications :

$$
\psi_{k}^{0}=\psi_{k}^{-} \circ\left(\psi_{k}^{+}\right)^{\circ(-1)} \text { et } \quad \psi_{k}^{\infty}=\psi_{k+1}^{+} \circ\left(\psi_{k}^{-}\right)^{\circ(-1)} \text {. }
$$

Proposition 3.16. Les applications $\psi_{k}^{0}$ et $\psi_{k}^{\infty}, k=0, \ldots, p-1$, ainsi construites satisfont aux propriétés suivantes :

1. $\psi_{k}^{0}$ est définie et injective sur un demi-plan inférieur $\operatorname{Im}(w)<-M<$ 0 et $\psi_{k}^{\infty}$ est définie et injective sur un demi-plan supérieur $\operatorname{Im}(w)>$ $M>0$,

2. $\psi_{k}^{0}(w+1)=\psi_{k}^{0}(w)+1$ et $\psi_{k}^{\infty}(w+1)=\psi_{k}^{\infty}(w)+1$, 
3. ces applications sont tangentes à des translations à l'infini :

$$
\lim _{\operatorname{Im}(w) \rightarrow+\infty} \psi_{k}^{0}(w)-w=\lambda_{k}^{0} \in \mathbb{C} \quad \text { et } \quad \lim _{\operatorname{Im}(w) \rightarrow-\infty} \psi_{k}^{\infty}(w)-w=\lambda_{k}^{\infty} \in \mathbb{C} .
$$

De plus, la partie euclidienne de l'atlas donnée par la composition de ces différentes translations nous redonne l'invariant formel $\lambda$ :

$$
\sum_{k=1}^{p} \lambda_{k}^{0}+\lambda_{k}^{\infty}=-\lambda
$$

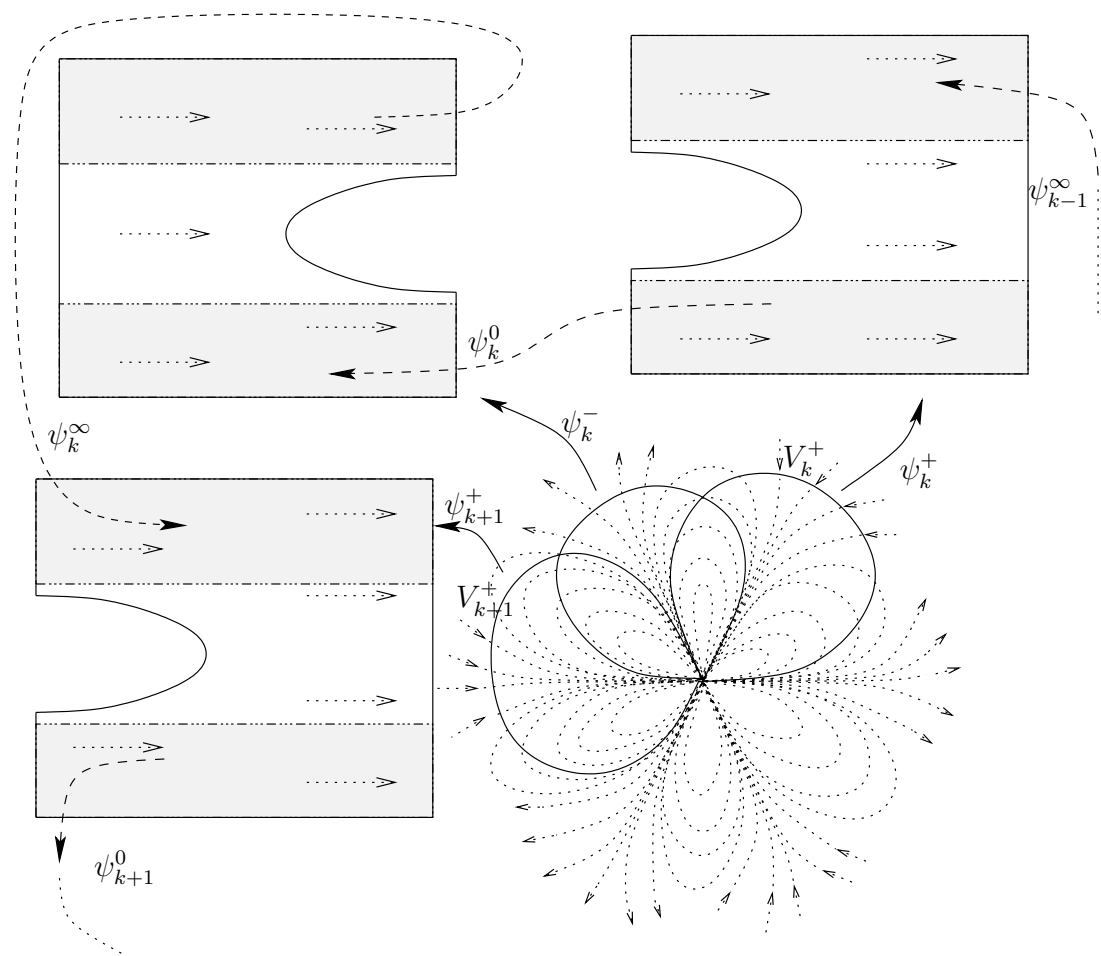

Figure 3.6 - L'atlas Euclidien

Il y a très peu d'arbitraire dans la construction de ces applications de transition : tout d'abord, le choix du pétale répulsif par lequel on a commencé la numérotation dépend de la partie linéaire de la coordonnée $z$ dans laquelle $f$ a été ramenée sous la forme $f(z)=z+z^{p+1}+\left(\frac{p+1}{2}-\frac{\lambda}{2 i \pi}\right) z^{2 p+1}+$ .... À partir de là, chaque coordonnée $\psi$ est unique à une constante additive près. Aussi, tout changement du système de coordonnées euclidiennes 
s'obtient comme composé de :

$$
\left(\tilde{w}_{k}^{+}, \tilde{w}_{k}^{-}\right)_{k}:=\left(w_{k+l}^{+}, w_{k+l}^{-}\right)_{k}, \quad l \in \mathbb{Z} / p \mathbb{Z},
$$

avec

$$
\left(\tilde{w}_{k}^{+}, \tilde{w}_{k}^{-}\right)_{k}:=\left(w_{k}^{+}+c_{k}^{+}, w_{k}^{-}+c_{k}^{-}\right)_{k}, \quad c_{k}^{ \pm} \in \mathbb{C} .
$$

Ces deux changements induisent respectivement les modifications d'écriture :

$$
\left(\tilde{\psi}_{k}^{0}, \tilde{\psi}_{k}^{\infty}\right)_{k}:=\left(\psi_{k+l}^{0}, \psi_{k+l}^{\infty}\right)_{k}
$$

et

$$
\left(\tilde{\psi}_{k}^{0}, \tilde{\psi}_{k}^{\infty}\right)_{k}:=\left(\psi_{k}^{0}\left(w-c_{k}^{+}\right)+c_{k}^{-}, \psi_{k}^{\infty}\left(w-c_{k}^{-}\right)+c_{k+1}^{+}\right)_{k}
$$

En jouant avec ces changements de coordonnées, on peut faire en sorte que tous les changements de cartes soient tangents à l'identité excepté un seul qui sera tangent à la translation $w \mapsto w-\lambda$.

Exemple 3.17. L'atlas défini par $\exp \left(X_{p, \lambda}\right)$ est euclidien en ce sens que les changements de cartes sont des translations : en choisissant les coordonnées euclidiennes obtenues par prolongement autour de $z=0$ d'une détermination de $\psi_{p, \lambda}$ sur $V_{0}^{+}$, les changements de cartes sont tous l'identité excepté $\psi_{p-1}^{\infty}(w)=w-\lambda$ puisque $\psi_{p-1}^{\infty} \circ \psi_{p, \lambda}\left(e^{2 i \pi} z\right)=\psi_{p, \lambda}(z)$. On récupère tous les atlas euclidiens de cette manière.

Finalement, la dynamique engendrée par le flot complexe de $X_{p, \lambda}$ peut se décrire comme suit. On relève, sur un revêtement à p feuillets, le pseudogroupe induit par les translations au voisinage de l'infini dans $\mathbb{C}$. Ensuite, on coupe l'anneau topologique ainsi obtenu puis on le recolle avec un décalage donné par $w \mapsto w+\lambda$ comme l'illustre la figure 3.7 dans le cas $p=1$; les dynamiques de translation passent au quotient puisqu'elles commutent au recollement.

De plus, on voit que la dynamique d'un grand commutateur

$$
\left[f^{\circ(-i N)}, f^{\circ(-N)}\right], \quad N>>0
$$

qui induit l'identité près de $z=0$, induit la transformation $f^{\circ \lambda}$ sur une autre composantes connexe de son domaine de définition. Plus généralement, dès que l'on ajoute à la dynamique de $f$ celle d'une itérée complexe $f^{\circ t}$ avec $t \notin \mathbb{R}$, alors le pseudo-groupe engendré contient aussi la dynamique de $f^{\circ \lambda}$ sur tout un voisinage de l'origine; en général, le pseudogroupe engendré est à orbites denses. 

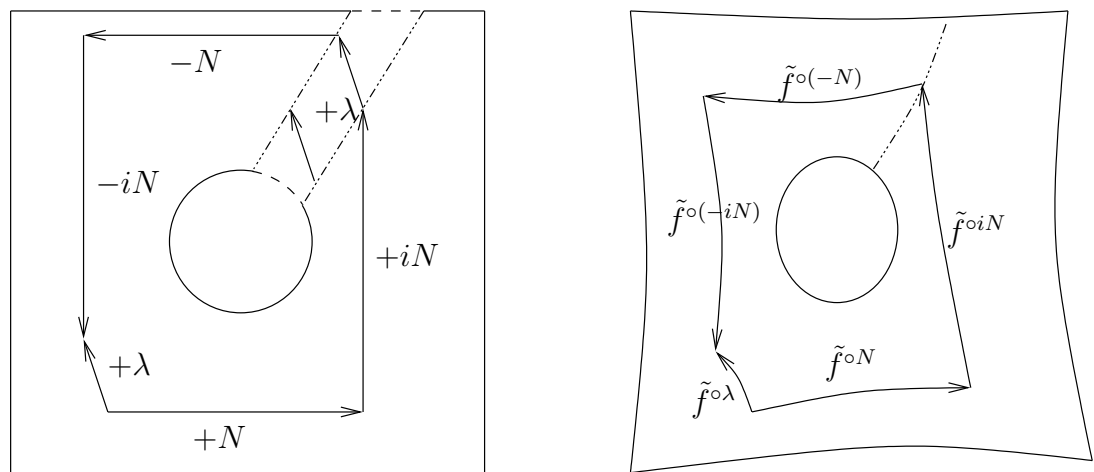

Figure 3.7 - Dynamique de $X_{1, \lambda}$ dans les variables $w=\psi_{1, \lambda}(z)$ puis $w=1 / z$

\subsection{Le chapelet de sphères}

Le quotient d'un voisinage épointé de l'origine par la dynamique s'obtient en recollant les différents quotients sectoriels $S_{k}^{ \pm}$par les applications :

$$
\begin{aligned}
\varphi_{k}^{0} & =\exp \left(-2 i \pi \psi_{k}^{0}\left(-\frac{\log (\tau)}{2 i \pi}\right)\right)=H_{k}^{-} \circ\left(H_{k}^{+}\right)^{\circ(-1)}: S_{k, 0}^{+} \mapsto S_{k, 0}^{-} \\
\varphi_{k}^{\infty} & =\exp \left(-2 i \pi \psi_{k}^{\infty}\left(-\frac{\log (\tau)}{2 i \pi}\right)\right)=H_{k+1}^{+} \circ\left(H_{k}^{-}\right)^{\circ(-1)}: S_{k, \infty}^{-} \mapsto S_{k+1, \infty}^{+}
\end{aligned}
$$

Proposition 3.18. Les applications $\varphi_{k}^{0}$ et $\varphi_{k}^{\infty}, k=0, \ldots, p-1$, ainsi construites satisfont aux propriétés suivantes :

1. $\varphi_{k}^{0}$ est un difféomorphisme d'un voisinage de $\tau=0$ dans $\mathbb{C}^{*}$ sur un voisinage de $\tau=0$ dans $\mathbb{C}^{*}$,

2. $\varphi_{k}^{\infty}$ est un difféomorphisme d'un voisinage de $\tau=\infty$ dans $\mathbb{C}^{*}$ sur un voisinage de $\tau=\infty$ dans $\mathbb{C}^{*}$;

En particulier, ces applications se prolongent holomorphiquement aux points respectifs $\tau=0$ et $\tau=\infty$ et induisent, dans le système de coordonnées linéaires $\left(\tau_{k}^{-}, \tau_{k}^{+}\right)_{k \in \mathbb{Z} / p \mathbb{Z}}$ choisi, des germes de difféomorphismes :

$$
\hat{\varphi}_{k}^{0} \in \operatorname{Diff}(\overline{\mathbb{C}}, 0) \quad \text { et } \quad \hat{\varphi}_{k}^{\infty} \in \operatorname{Diff}(\overline{\mathbb{C}}, \infty) .
$$

Ce quotient est appelé chapelet de sphères dans [139].

Remarque 3.19. Seuls les germes $\hat{\varphi}_{k}^{0}$ et $\hat{\varphi}_{k}^{\infty}$ sont vraiment bien définis par la dynamique de $f$, toute diminution de l'ouvert $U$ ayant pour exacte conséquence de diminuer de façon comparable la taille de ces recollements. 


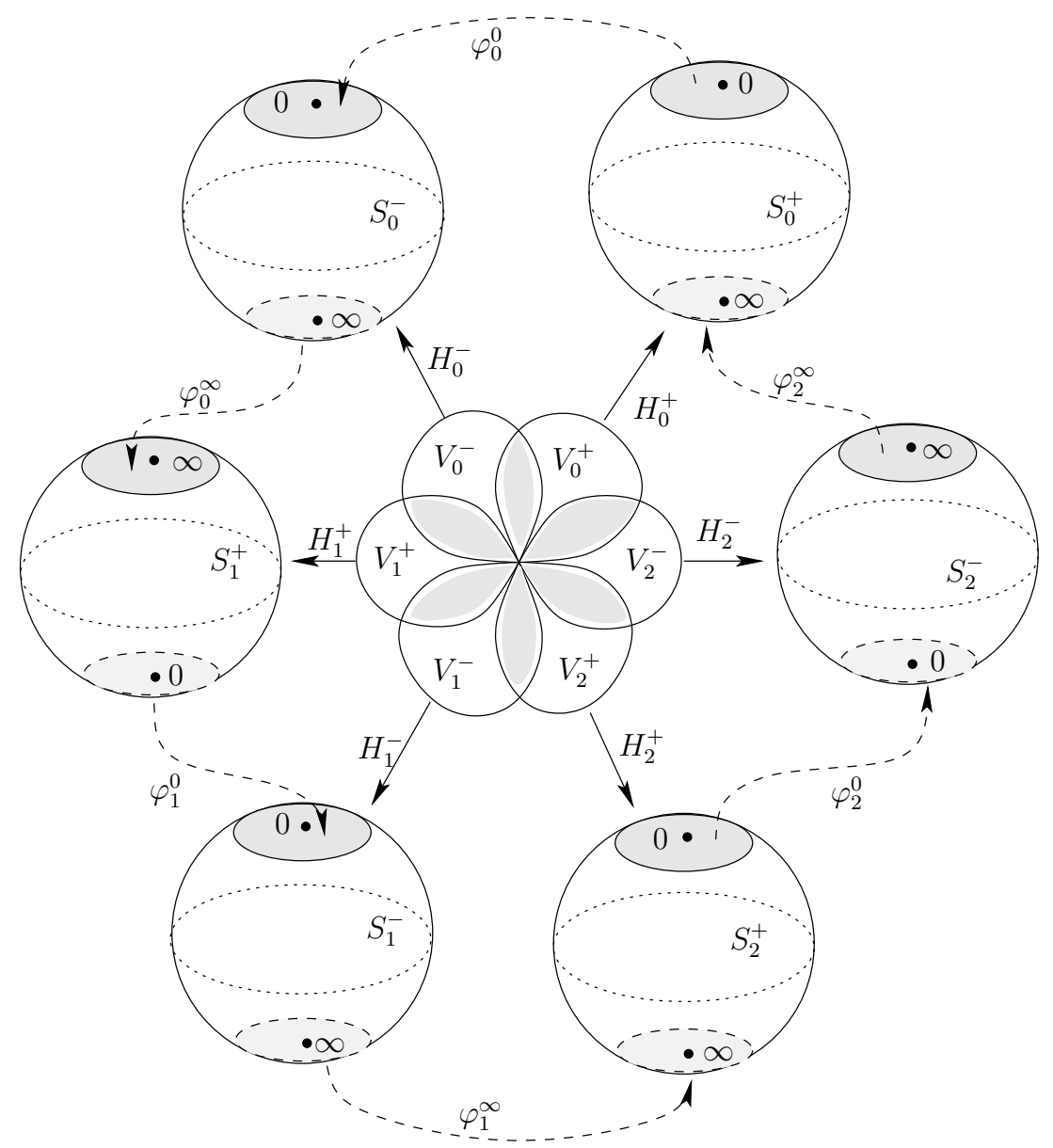

FiguRE 3.8 - Le chapelet de sphères 
Tout changement du système de coordonnées linéaires s'obtient comme composé de :

$$
\left(\tilde{\tau}_{k}^{+}, \tilde{\tau}_{k}^{-}\right)_{k}:=\left(\tau_{k+l}^{+}, \tau_{k+l}^{-}\right)_{k}, \quad l \in \mathbb{Z} / p \mathbb{Z},
$$

avec

$$
\left(\tilde{\tau}_{k}^{+}, \tilde{\tau}_{k}^{-}\right)_{k}:=\left(\gamma_{k}^{+} \tau_{k}^{+}, \gamma_{k}^{-} \tau_{k}^{-}\right)_{k}, \quad \gamma_{k}^{ \pm} \in \mathbb{C} .
$$

Ces changements induisent respectivement les modifications d'écriture :

$$
\left(\tilde{\varphi}_{k}^{0}, \tilde{\varphi}_{k}^{\infty}\right)_{k}:=\left(\varphi_{k+l}^{0}, \varphi_{k+l}^{\infty}\right)_{k},
$$

et

$$
\left(\tilde{\varphi}_{k}^{0}, \tilde{\varphi}_{k}^{\infty}\right)_{k}:=\left(\gamma_{k}^{-} \varphi_{k}^{0}\left(\tau / \gamma_{k}^{+}\right), \gamma_{k+1}^{+} \varphi_{k}^{\infty}\left(\tau / \gamma_{k}^{-}\right)\right)_{k} .
$$

En jouant avec ces changements de coordonnées, on peut faire en sorte que tous les recollements soient tangents à l'identité excepté $\varphi_{p}^{\infty}$ qui sera tangent à la transformation linéaire $\tau \mapsto e^{2 i \pi \lambda} \tau$, où $\lambda$ n'est autre que l'invariant formel de $f$; c'est la partie linéaire du chapelet.

L'ordre cyclique du chapelet et la polarité \pm assignée à chaque sphère sont bien définis par sa construction. On précisera éventuellement chapelet polarisé orienté et, lorsque l'on ne s'interessera qu'à l'espace analytique sous-jacent, on parlera alors de chapelet géométrique. Se donner un chapelet polarisé orienté, c'est encore se donner une collection :

$$
\left(\hat{\varphi}_{k}^{0}, \hat{\varphi}_{k}^{\infty}\right)_{k} \in(\operatorname{Diff}(\overline{\mathbb{C}}, 0) \times \operatorname{Diff}(\overline{\mathbb{C}}, \infty))^{p}
$$

modulo les changements du système de coordonnées linéaires que nous venons de décrire; se donner le chapelet géométrique associé c'est s'autoriser, en plus des changements précédents, le renversement de l'orientation :

$$
\left.\left(\tilde{\varphi}_{k}^{0}, \tilde{\varphi}_{k}^{\infty}\right)_{k}:=\left(1 /\left(\varphi_{p-k}^{\infty}\right)^{\circ(-1)}(1 / \tau), 1 / \varphi_{p-k}^{0}\right)^{\circ(-1)}(1 / \tau)\right)_{k},
$$

et le renversement de la polarité :

$$
\left(\tilde{\varphi}_{k}^{0}, \tilde{\varphi}_{k}^{\infty}\right)_{k}:=\left(1 /\left(\varphi_{k}^{\infty}\right)(1 / \tau), 1 /\left(\varphi_{k+1}^{0}\right)(1 / \tau)\right)_{k} .
$$

Les difféomorphismes $f$ et $f^{\circ(-1)}$ ont bien sûr même quotient, c'est-àdire même chapelet géométrique; leur chapelet polarisé orienté se correspondent simplement par un renversement de la polarité puisque les pétales attractifs deviennent répulsifs et vice-versa.

\subsection{Les invariants holomorphes}

Théorème 3.20 (Birkhoff,Écalle). Soient $f, g \in \operatorname{Diff}(\mathbb{C}, 0)$ tous deux tangents à l'identité à l'ordre $p$ de même invariant formel $\lambda$. Alors sont équivalents : 
1. $f$ et $g$ sont analytiquement conjugués,

2. $f$ et $g$ ont même atlas de trivialisation (i.e. les changements de cartes $\left(\psi_{k}^{0}, \psi_{k}^{\infty}\right)_{k}$ respectifs sont les mêmes dans un bon système de coordonnées euclidiennes, à la taille près de leur domaine de définition),

3. $f$ et $g$ ont même chapelet polarisé orienté (i.e. les germes de recollement $\left(\hat{\varphi}_{k}^{0}, \hat{\varphi}_{k}^{\infty}\right)_{k}$ respectifs sont les mêmes dans un bon système de coordonnées linéaires).

Démonstration. L'équivalence $(2) \Leftrightarrow(3)$ est claire.

L'implication $(1) \Rightarrow(3)$ est démontrée "par construction" mais donnons l'argument suivant dont nous allons abuser par la suite. Si $\varphi$ envoie la dynamique de $f$ sur celle de $g$, alors $\varphi$ passe au quotient pour définir un difféomorphisme analytique $\bar{\varphi}$ entre les chapelets respectifs de $f$ et de $g$. Puisque $\varphi$ respecte l'orientation du plan et envoie pétales attractifs de $f$ sur pétales attractifs de $g, \bar{\varphi}$ respecte l'orientation et la polarité des chapelets. Enfin, $\bar{\varphi}$ envoie biholomorphiquement chaque sphère épointée de $f$ sur une sphère épointée de $g$ ( $\varphi$ respecte les pétales) et doit donc, dans les coordonnées respectives, être linéaire : $\bar{\varphi}$ n'est autre qu'un changement du système de coordonnées linéaires du chapelet polarisé orienté de $f$.

Pour la réciproque $(3) \Rightarrow(1)$, s'il existe un changement système de coordonnées linéaires identifiant les chapelets polarisés orientés de $f$ et de $g$, c'est-à-dire un difféomorphime $\bar{\varphi}$ entre les deux quotients, alors, même si le passage au quotient par les dynamiques de $f$ et de $g$ n'est pas tout à fait un revêtement, le difféomorphisme $\bar{\varphi}$ se relève en un difféomorphisme sur $U \backslash\{0\}$ envoyant par construction la dynamique de $f$ sur celle de $g$ (transformations du revêtement) qui se prolonge à l'origine par Riemann. Le relèvement intermédiaire de $\bar{\varphi}$ à l'atlas trivialisant se fait sans monodromie puisque l'on a pris garde de demander à ce que les invariants formels $\lambda$ respectifs coïncident.

En particulier, on déduit immédiatement le :

Corollaire 3.21. Soit $f(z)=z+\cdots \in \operatorname{Diff}(\mathbb{C}, 0)$ d'invariant formel $\lambda$. Alors sont équivalents :

- $f$ est analytiquement conjuguée à $\exp \left(X_{p, \lambda}\right)$,

- l'atlas de trivialisation de $f$ est euclidien,

- le chapelet de $f$ est linéaire,

- $f$ est plongeable dans un groupe à un paramètre.

Le théorème suivant était conjecturé par Birkhoff.

Théorème 3.22 (Écalle,Malgrange,Voronin). Étant donné un chapelet polarisé orienté, c'est-à-dire une collection

$$
\left(\hat{\varphi}_{k}^{0}, \hat{\varphi}_{k}^{\infty}\right)_{k} \in(\operatorname{Diff}(\overline{\mathbb{C}}, 0) \times \operatorname{Diff}(\overline{\mathbb{C}}, \infty))^{p}
$$


et un choix de logarithme $\lambda \in \mathbb{C}$ pour la partie linéaire, alors il existe un difféomorphisme tangent à l'identié $f \in \operatorname{Diff}(\mathbb{C}, 0)$ d'invariant $\lambda$ qui réalise ce chapelet.

Je tiens à remercier Xavier Buff qui m'a aidé à trouver l'argument élémentaire permettant de conclure la preuve qui suit.

Démonstration. On commence par relever les germes de difféomorphismes en une collection d'applications $\left(\psi_{k}^{0}, \psi_{k}^{\infty}\right)_{k}$ satisfaisant aux propriétés 1-3 de la Proposition 3.16 par :

$$
\varphi_{k}^{0}=\exp \left(-2 i \pi \psi_{k}^{0}\left(-\frac{\log (\tau)}{2 i \pi}\right)\right) \quad \text { et } \quad \varphi_{k}^{\infty}=\exp \left(-2 i \pi \psi_{k}^{\infty}\left(-\frac{\log (\tau)}{2 i \pi}\right)\right) .
$$

On déduit, en renversant notre construction, un atlas définissant une dynamique conforme sur un anneau topologique. La difficulté est de montrer que cet anneau est conformément équivalent à un voisinage épointé de $0 \in \mathbb{C}$, c'est-à-dire que le bout $w=\infty$ est parabolique. Si c'est le cas, alors la dynamique se prolonge en 0 par Riemann et c'est un germe de difféomorphisme puisqu'il est bijectif près de 0 . Pour une raison d'indice, la dynamique est alors celle d'un élément $f \in \operatorname{Diff}(\mathbb{C}, 0)$ tangent à l'identité à l'ordre $p$ exactement; par construction, il réalise les invariants donnés.

Pour montrer que l'atlas construit est parabolique vers $w=\infty$ nous allons procéder par l'absurde et supposer que c'est un anneau de module fini, donc uniformisable sur l'anneau standart $A=\{r<|z|<1\}$ avec $0<r<1$. Le bord correspondant à $w=\infty$ dans l'atlas est ici le bord intérieur $C_{r}=\{|z|=r\}$ (voir figure 3.9). On note $f$ la dynamique sur $A$ : $f$ est bien définie près de $C_{r}$ qui est invariant par $f$. Par le Principe de réflexion de Schwarz, $f$ s'étend analytiquement au voisinage de $C_{r}$ et induit un difféomorphisme analytique du cercle $C_{r} \rightarrow C_{r}$. Nous allons montrer que $f$ est l'identité sur $C_{r}$ aboutissant ainsi à une contradiction. Il suffit pour cela de montrer que la distance (euclidienne) entre $z$ et $f(z)$ tend vers 0 lorsque $z \in A$ tend vers le bord $C_{r}$, ou encore que leur distance hyperbolique est bornée pour la métrique de Poincaré sur l'anneau. Nous allons majorer cette dernière dans les cartes de l'atlas. Fixons $R>1$ et considérons, dans chaque carte $\tilde{V}$ et pour chaque point $w \in \tilde{V}$ à distance au moins $R$ du bord intérieur, le plongement affine

$$
\phi: \mathbb{D} \rightarrow \tilde{V} ; t \mapsto w+R t
$$

du disque unité dans $\tilde{V}$. La distance entre $\phi^{-1}(w)=0$ et son image par la dynamique $\phi^{-1}(w+1)=1 / R$ est $1 / R<1$; la distance hyperbolique dans $\mathbb{D}$ pour ces mêmes points est majorée par une constante $c(R)$. Une conséquence du Lemme de Schwarz est que cette distance majore celle des images $z$ et $f(z)$ pour la métrique hyperbolique de l'anneau. L'ensemble des points $z$ pour lesquels on a obtenu la majoration forment visiblement un voisinage du bord $C_{r}$. 


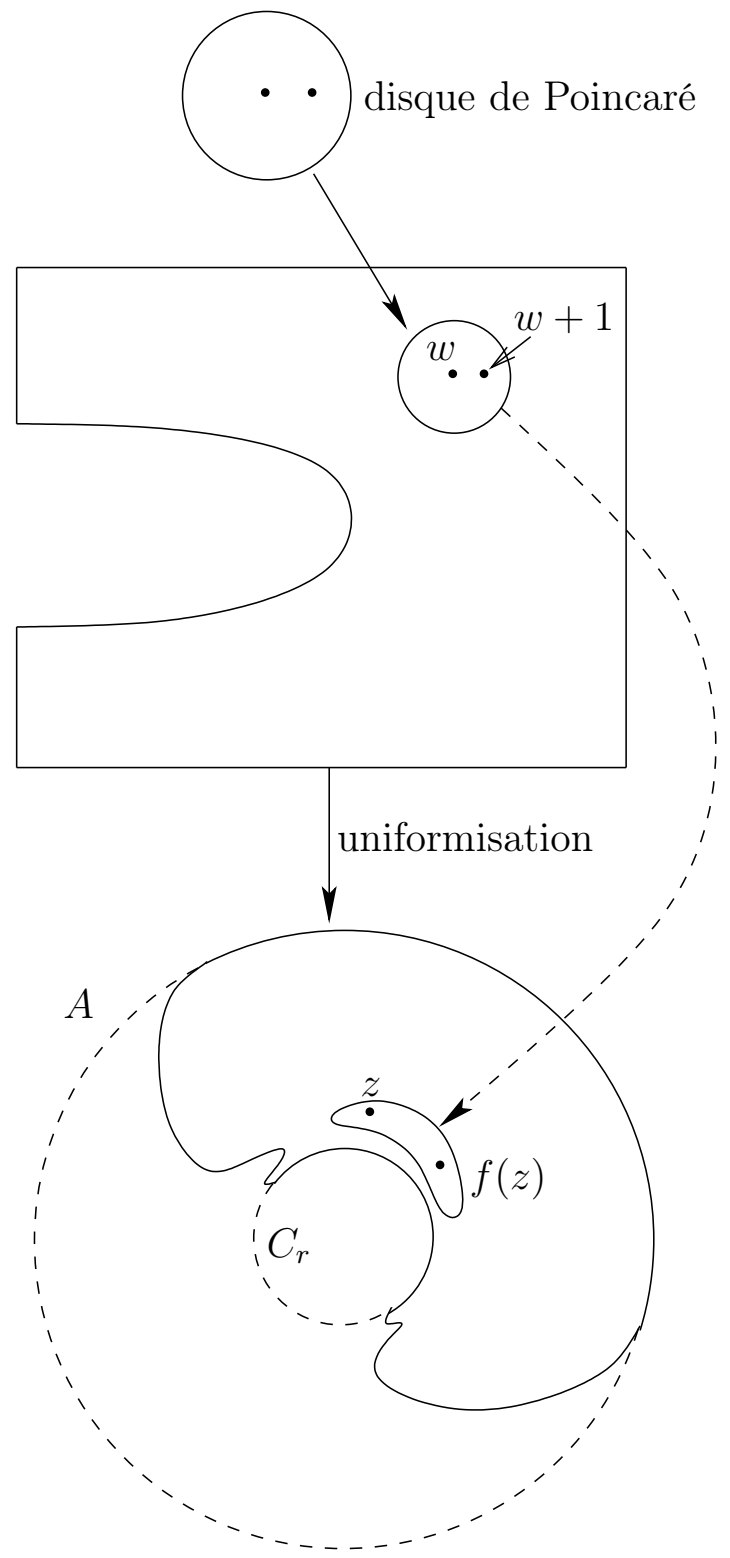

Figure 3.9 - Estimation de la vitesse de la dynamique dans la métrique hyperbolique 


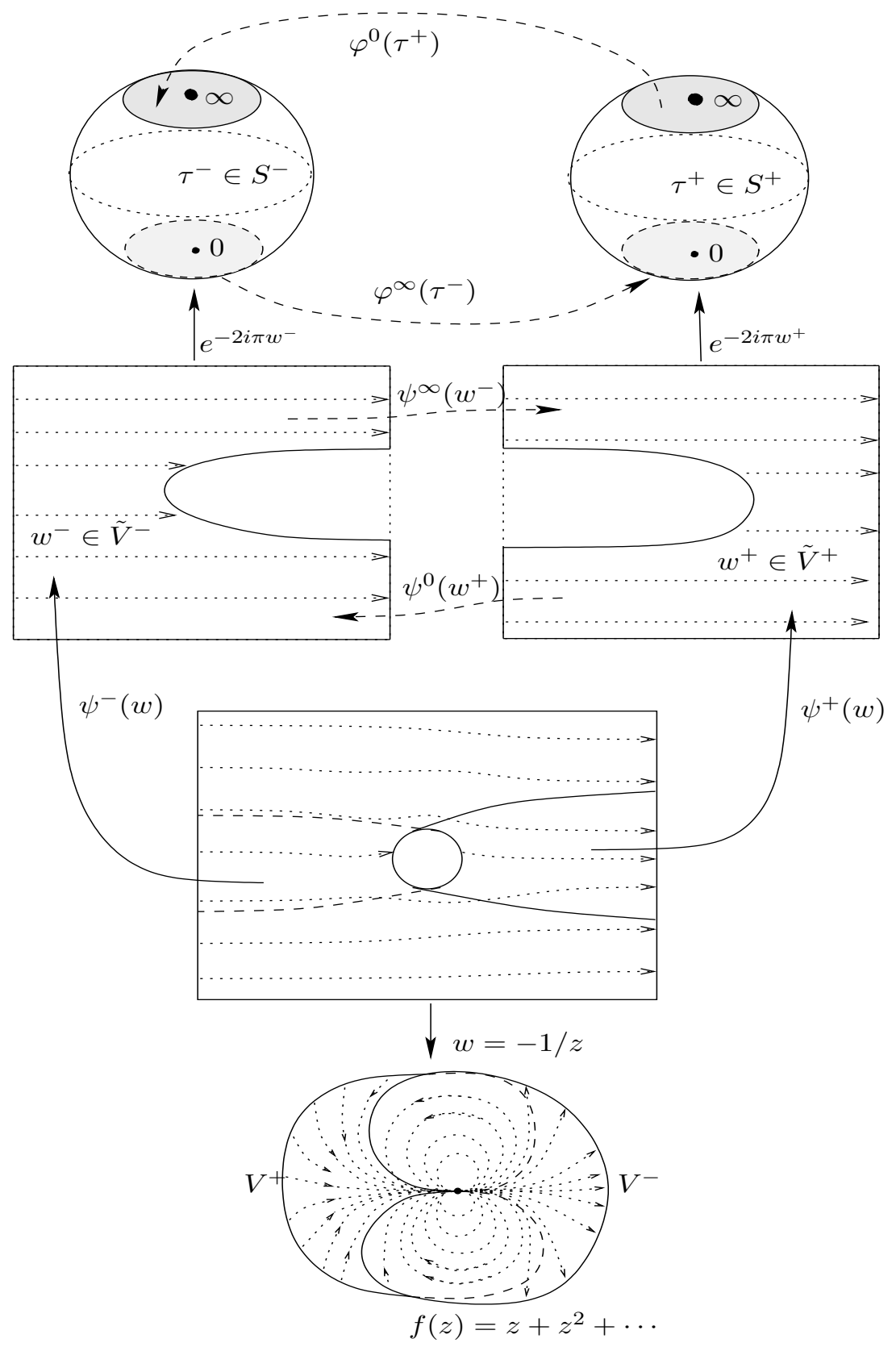

FiguRE 3.10 - Récapitulatif de la construction des invariants analytiques 
Nous résumons dans la figure 3.10 la construction des invariants dans le cas $p=1$. Donnons maintenant quelques exemples classiques de difféomorphismes tangents à l'identité dont les invariants sont non triviaux.

Exemple 3.23 (Birkhoff). La solution formelle $\hat{\phi}$ à l'infini de l'équation aux différences

$$
\phi(z+1)-\phi(z)=\frac{1}{z^{n}}
$$

est divergente pour tout $n \geq 2$. En effet, il est bien connu que la série de Stirling asymptote à l'infini à la fonction $\Gamma(z)$ diverge; elle satisfait l'équation fonctionnelle $\hat{\Gamma}(z+1)=z \hat{\Gamma}(z)$ et $\hat{\phi}$ est donnée pour $n=1$ par $\hat{\phi}(z)=\frac{\partial}{\partial z} \log (\hat{\Gamma}(z))$ et pour $n$ quelconque par dérivation de cette dernière.

La normalisante formelle $\hat{\varphi}_{c}$ de l'automorphisme $f_{c}(z)=z /\left(1-z-c z^{3}\right)$ diverge pour des valeurs de c arbitrairement proches de 0. En effet, si tel n'était pas le cas, $\varphi_{c}(z)$ serait une fonction holomorphe de deux variables $c$ et $z$ au voisinage du point $(c, z)=(0,0)$. La transformation $\tilde{\varphi}_{c}$ lui correspondant par $z \mapsto \frac{-1}{z}$ satisferait alors identiquement l'équation d'Abel à un paramètre $\tilde{\varphi}_{c}\left(z+1+\frac{c}{z^{2}}\right)=\tilde{\varphi}_{c}(z)+1$. Un calcul immédiat montre que $\tilde{\varphi}_{c}(z)=z+c\left(\frac{1}{z}+\cdots\right) \in \mathbb{C}[c][[z]]$; en dérivant par rapport à $c$, on obtient pour $c=0$ :

$$
\phi(z+1)-\phi(z)=\frac{-1}{z^{2}} \quad \text { ò̀ } \quad \phi(z)=\left[\frac{\partial}{\partial c} \tilde{\varphi}_{c}(z)\right]_{c=0} .
$$

Cependant, comme nous l'avons vu plus haut, cette équation n'admet pas de solution convergente à l'infini.

Exemple 3.24 ([4]). Le germe en $0 \in \mathbb{C}$ défini par le polynôme $f(z)=$ $z+z^{2}+z^{3}$ n'est pas normalisable, i.e. n'est pas analytiquement conjugué $\grave{a}$ sa forme normale (formelle) $h_{1,0}(z)=z /(1-z)$. En effet, supposons qu'il existe $\varphi \in \operatorname{Diff}(\mathbb{C}, 0)$ convergeant sur un petit disque $U \subset \mathbb{C}$ tel que $\varphi \circ h_{1,0}=f \circ \varphi ;$ pour tout point $z \in \overline{\mathbb{C}}=\mathbb{C} \cup\{\infty\}$, il existe un entier positif $n \in \mathbb{N}$ tel que $z=h_{1,0}^{\circ n}\left(z^{\prime}\right) \in U$; on définit $\varphi(z)$ par $: \varphi(z)=\varphi \circ h_{1,0}^{\circ n}\left(z^{\prime}\right)=$ $f^{\circ} \circ \varphi\left(z^{\prime}\right)$; ceci a toujours un sens car $f$ est polynomiale (et ses itérées positives sont toutes définies sur $\mathbb{C}$ ); ainsi, $\varphi$ se prolonge sur $\overline{\mathbb{C}}$ et est constante par Liouville, contradiction.

\subsection{Les symétries du chapelet}

Pour toute la suite, choisissons, sur le chapelet, un système de coordonnées linéaires homogènes, c'est-à-dire tel que chaque recollement $\varphi_{k}^{0}$ et $\varphi_{k}^{\infty}$ soit tangent à $\tau \mapsto e^{-2 i \pi \lambda / 2 p} \tau$. 


\subsubsection{Symétries holomorphes et centralisateur de $f$ $([70,225])$}

On appelle centralisateur de $f$ dans $\operatorname{Diff}(\mathbb{C}, 0)$ et on note $\operatorname{Cent}(f)$ le sous-groupe des éléments de $\operatorname{Diff}(\mathbb{C}, 0)$ qui commutent à $f$.

On appelle symétrie holomorphe du chapelet tout difféomorphisme holomorphe du chapelet géométrique respectant la polarité et l'orientation, c'est-à-dire tout changement du système de coordonnées linéaires laissant invariante l'écriture des recollements $\left(\hat{\varphi}_{k}^{0}, \hat{\varphi}_{k}^{\infty}\right)_{k}$. Un élément de $g \in$ Cent $(f)$ induit par passage au quotient un tel difféomorphisme :

$$
\operatorname{Cent}(f) \rightarrow \operatorname{Diff}((\mathbb{C}, 0) / f) ; g \mapsto \bar{g}
$$

Cette flêche est surjective et son noyau est engendré par $f$; le groupe Cent $(f) / f$ s'identifie ainsi au groupe des difféomorphismes du chapelet polarisé orienté. Précisons cette correspondance.

Fixons $\hat{\varphi}$ une coordonnée normalisante formelle :

$$
\hat{\varphi}_{*} f=\exp \left(X_{p, \lambda}\right) .
$$

D'après la Proposition 2.17, Cent $(f)$ s'identifie au sous-groupe des éléments de :

$$
\hat{\varphi}^{*}\left\{e^{2 i \pi k / p} \exp \left(t X_{p, \lambda}\right) ;(k, t) \in \mathbb{Z} / p \mathbb{Z} \times \mathbb{C}\right\}
$$

qui convergent. Par ailleurs, dans un système de coordonnées linéaires homogènes sur le chapelet, les contraintes linéaires de recollement montrent que tout difféomorphisme du chapelet est de la forme :

$$
\left(\tau_{k}^{-}, \tau_{k}^{+}\right)_{k} \mapsto\left(\gamma \tau_{k+l}^{-}, \gamma \tau_{k+l}^{+}\right)_{k}
$$

où $(l, \gamma) \in \mathbb{Z} / p \mathbb{Z} \times \mathbb{C}^{*}$.

Théorème 3.25. Sont équivalents :

1. $\hat{\phi}^{*}\left(e^{2 i \pi l / p} \exp \left(t X_{p, \lambda}\right)\right)$ converge,

2. $\left(\tau_{k}^{-}, \tau_{k}^{+}\right)_{k} \mapsto\left(e^{2 i \pi t} \tau_{k+l}^{-}, e^{2 i \pi t} \tau_{k+l}^{+}\right)_{k}$ est un difféomorphisme du chapelet.

Démonstration. La première assertion nous dit qu'il existe un élément $g(z)=e^{2 i \pi l / p}\left(z+t z^{p+1}+\cdots\right) \in \operatorname{Diff}(\mathbb{C}, 0)$ qui commute à $f$ et donc définit un difféomorphisme $\bar{g}$ du chapelet. Un développement limité utilisant l'asymptotique des trivialisantes sectorielles $\psi$ permet d'identifier $\bar{g}$ comme étant précisément le difféomorphisme décrit par la seconde assertion. Réciproquement, c'est un jeu d'écriture que de vérifier que toute symétrie du chapelet se relève en une symétrie de la dynamique initiale. 
Rappelons (voir Proposition 2.17) que $f=\exp (X)$ pour un unique champ de vecteurs formel $X \in \widehat{\mathcal{X}}(\mathbb{C}, 0)$, et que par suite, ses itérées formelles $f^{\circ t}:=\exp (t X)$ sont bien définies pour tout $t \in \mathbb{C}$. Notons $T \subset \mathbb{C}$ le sous-groupe des $t$ pour lesquels $f^{\circ t} \in \operatorname{Diff}(\mathbb{C}, 0)$ converge. Bien sûr, les itérées de $f$ convergent et $\mathbb{Z} \subset T$. Alors J. Écalle et O. Liverpool ont indépendamment démontré le :

Corollaire 3.26 (Écalle,Liverpool). Soient $f, X$ et $T$ comme ci-dessus. Alors

- ou bien $T=\mathbb{C}$ et $f$ est analytiquement conjugué à $\exp \left(X_{p, \lambda}\right)$,

- ou bien $T=\frac{1}{n} \mathbb{Z}$ pour un $n \in \mathbb{N}^{*}$ convenable.

Plus précisément, sont équivalents :

- la racine $n^{\text {ème }} f^{\circ(1 / n)}$ est bien définie, $n \in \mathbb{N}^{*}$,

- les germes $\varphi_{k}^{0}$ et $\varphi_{k}^{\infty}$ sont tous du type $\tau \varphi\left(\tau^{n}\right)$,

Démonstration. On applique le Théorème 3.25 à $f$ avec $l=0$ et $t=$ $1 / n$.

Corollaire 3.27. On a l'alternative suivante:

- ou bien $f$ est analytiquement conjugué à $\exp \left(X_{p, \lambda}\right)$ et

$$
\operatorname{Cent}(f) \simeq\left\{e^{2 i \pi k / p} \exp \left(t X_{p, \lambda}\right) ;(k, t) \in \mathbb{Z} / p \mathbb{Z} \times \mathbb{C}\right\}
$$

- ou bien le groupe Cent $(f)$ est d'indice fini sur le sous-groupe engendré par $f$.

Démonstration. Dès que le chapelet est non linéaire, son groupe de difféomorphismes est fini. En effet, le sous-groupe des symétries qui fixent les sphères du chapelet est d'indice fini (au plus $p$ ) et il est lui-même fini d'après le Corollaire 3.26. Par suite Cent $(f) / f$ est aussi fini.

Corollaire 3.28. Soit $1 \leq l \leq p$ un entier divisant $p$. Alors sont équivalents :

- $f$ est, dans une bonne coordonnée conforme, de la forme $z \tilde{f}\left(z^{p / l}\right)$,

- il existe $g \in \operatorname{Diff}(\mathbb{C}, 0)$ périodique d'ordre $l$ et commutant à $f$,

- on $a \varphi_{k+l}^{0}=\varphi_{k}^{0}$ et $\varphi_{k+l}^{\infty}=\varphi_{k}^{\infty}$ pour tout $k \in \mathbb{Z} / p \mathbb{Z}$.

Démonstration. On applique le Théorème 3.25 à $f$ avec $t=0$.

\subsubsection{Difféomorphismes résonants}

On appelle difféomorphisme résonant un élément $f \in \operatorname{Diff}(\mathbb{C}, 0)$ non périodique dont la partie linéaire est une racine de l'unité :

$$
f(z)=e^{2 i \pi p / q} z+z^{k q}+\cdots \quad \text { avec } \quad p, q, k \in \mathbb{N}^{*} .
$$


L'étude de $f$ se ramène alors à celle d'un difféomorphisme tangent à l'identité $f^{\circ q}$ muni d'une racine $q^{\text {ème }}$. Notamment, le quotient de $f$ s'obtient en quotientant le chapelet de $2 k q$ sphères de $f^{\circ q}$ par son difféomorphisme $\bar{f}$ et est encore un chapelet polarisé orienté mais ne possédant plus que $2 k$ sphères.

Remarque 3.29. Contrairement à ce que l'on a pu penser, un tel difféomorphisme peut rarement se ramener sous la forme $f(z)=z \tilde{f}\left(z^{q}\right)$ par changement de coordonnée conforme. En effet, ceci signifierait qu'il existe sur le chapelet de $2 k q$ sphères associé à $f^{\circ q}$ deux symétries indépendantes rendant compte respectivement de l'existence d'une racine $q^{\grave{e} m e}$, à savoir $f$, et de l'existence d'un difféomorphisme périodique d'ordre q dans le centralisateur de $f^{\circ q}$, à savoir la rotation $z \mapsto e^{2 i \pi / q} z$ qui commute à l'écriture de $f$. On vérifie aisément que les conditions d'existence respectives de telles symétries sur le chapelet sont indépendantes : l'existence de l'une n'implique absolument pas l'existence de l'autre.

\subsubsection{Anti-symétries holomorphes du chapelet ([226, 58])}

On appelle anti-symétrie holomorphe du chapelet tout difféomorphisme holomorphe du chapelet géométrique qui respecte l'orientation mais renverse la polarité. Dans un système de coordonnées linéaires homogènes du chapelet, les contraintes linéaires de recollement font qu'une telle transformation s'écrit :

$$
\left(\tau_{k}^{+}, \tau_{k}^{-}\right)_{k} \mapsto\left(\frac{\gamma}{\tau_{k+l}^{-}}, \frac{\gamma}{\tau_{k+l+1}^{+}}\right)_{k},
$$

pour des constantes $\gamma \in \mathbb{C}^{*}$ et $l \in \mathbb{Z} / p \mathbb{Z}$.

Théorème 3.30. Sont équivalents :

- les dynamiques de $f$ et $f^{\circ(-1)}$ sont conjuguées par un élément $g \in$ $\operatorname{Diff}(\mathbb{C}, 0)$ :

$$
g^{\circ(-1)} \circ f \circ g=f^{\circ(-1)},
$$

$-\lambda=0$ et le chapelet possède une anti-symétrie holomorphe.

Dans ce cas, $g$ est périodique et $g^{\circ 2} \in \operatorname{Cent}(f)$.

Démonstration. Un tel élément $g$ définit visiblement, par passage au quotient, une anti-symétrie holomorphe $\bar{g}$ du chapelet. La présence d'une telle symétrie implique en particulier que la partie linéaire $e^{-2 i \pi \lambda}$ du chapelet est triviale, i.e. que $\lambda \in \mathbb{Z}$. Reciproquement, une telle symétrie se relève sur l'atlas trivialisant, et donc définit un élément $g \in \operatorname{Diff}(\mathbb{C}, 0)$ conjugant $f$ à $f^{\circ(-1)}$ dès que $\lambda=0$. Le fait que $g^{\circ 2} \in C e n t(f)$ est évident et le fait que $g$ soit périodique peut se voir par l'absurde : si $g$ n'est pas périodique, alors il existe un entier $n \in \mathbb{Z}^{*}$ tel que $g^{\circ n}$ soit tangent à l'identité et donc 
une itérée complexe de $f$; maintenant, $g$ conjugue $g^{\circ n}$ à son inverse, ce qui n'est possible que si $g^{\circ n}=$ identité.

\subsubsection{Symétries anti-holomorphes du chapelet}

On appelle symétrie anti-holomophe du chapelet tout difféomorphisme anti-holomorphe du chapelet géométrique préservant la polarité des sphères. Dans un système de coordonnées linéaires homogènes du chapelet, les contraintes linéaires de recollement font qu'une telle transformation s'écrit :

$$
\left(\tau_{k}^{+}, \tau_{k}^{-}\right)_{k} \mapsto\left(\frac{\gamma}{\bar{\tau}_{2 l-k}^{+}}, \frac{\gamma}{\bar{\tau}_{2 l-k-1}^{-}}\right)_{k}
$$

$\mathrm{ou}$

$$
\left(\tau_{k}^{+}, \tau_{k}^{-}\right)_{k} \mapsto\left(\frac{\gamma}{\bar{\tau}_{2 l-k-1}^{+}}, \frac{\gamma}{\bar{\tau}_{2 l-k}^{-}}\right)_{k}
$$

pour des constantes $\gamma \in \mathbb{C}^{*}$ et $l \in \mathbb{Z} / p \mathbb{Z}$.

On parlera de symétrie involutive anti-holomorphe lorsque cette transformation est une involution, i.e. lorsque $\gamma$ est réel.

Théorème 3.31. Sont équivalents :

- il existe une transformation anticonforme $\sigma$ fixant $0 \in \mathbb{C}$ (i.e. $\sigma=$ $g(\bar{z})$ avec $g \in \operatorname{Diff}(\mathbb{C}, 0)$ ) et commutant à $f$,

- le chapelet possède une symétrie anti-holomorphe.

Si c'est le cas, alors $\frac{\lambda}{2 i \pi} \in \mathbb{R}$ et $\sigma^{\circ 2} \in \operatorname{Cent}(f)$.

Corollaire 3.32. Supposons $\frac{\lambda}{2 i \pi} \in \mathbb{R}$. Alors sont équivalents :

- il existe une coordonnée conforme dans laquelle $f$ est réel, i.e. $f \in$ $\operatorname{Diff}(\mathbb{R}, 0)$,

- il existe une courbe lisse analytique réelle à l'origine invariante par $f$,

- il existe une involution anti-holomorphe $\sigma$ fixant $0 \in \mathbb{C}$ et commutant $\grave{a} f$,

- le chapelet possède une symétrie involutive anti-holomorphe.

Dès que $\frac{\lambda}{2 i \pi} \in \mathbb{R}, f$ est formellement conjugué à un élément de $\operatorname{Diff}(\mathbb{R}, 0)$, à savoir $\exp \left(X_{p, \lambda}\right)$; en particulier, $f$ commute à une "involution antiholomorphe formelle". D'après le Corollaire précédent, cette involution diverge en général et $f$ n'est pas réel dans aucune coordonnée conforme. Par contre, les flots sectoriels $X_{k}^{ \pm}=\left(\psi_{k}^{ \pm}\right)^{*} \partial_{w}$ nous fournissent un système complet de trajectoires analytiques réelles dans chaque pétale qui, chacune, se prolonge de manière $C^{\infty}$ en 0 grâce au développement asymptotique des trivialisations sectorielles. Le Corollaire précédent nous décrit les conditions nécessaires et suffisantes pour que l'une de ces trajectoires 
soit analytique à l'origine. Notons que le difféomorphisme est analytiquement normalisable dès que deux de ces trajectoires sont analytiques (dans un même pétale); en effet, en composant les involutions de Schwarz associées, on récupère un élément du centralisateur de $f$ qui est tangent à l'identité mais visiblement pas une itérée réelle de $f$ puisqu'il permute ses trajectoires réelles.

Lorsque $\frac{\lambda}{2 i \pi} \notin \mathbb{R}$, on peut encore construire des trajectoires analytiques dans les pétales comme ci-dessus, mais leur classe de différentiabilité à l'origine est finie.

\subsubsection{Anti-symétries anti-holomorphes du chapelet $([152,221])$}

On appelle anti-symétrie anti-holomophe du chapelet tout difféomorphisme anti-holomorphe du chapelet géométrique inversant la polarité des sphères. Dans un système de coordonnées linéaires homogènes du chapelet, les contraintes linéaires de recollement font qu'une telle transformation s'écrit :

$$
\left(\tau_{k}^{+}, \tau_{k}^{-}\right)_{k} \mapsto\left(\gamma \bar{\tau}_{2 l-k}^{-}, \gamma \bar{\tau}_{2 l-k}^{+}\right)_{k}
$$

$\mathrm{ou}$

$$
\left(\tau_{k}^{+}, \tau_{k}^{-}\right)_{k} \mapsto\left(\gamma \bar{\tau}_{2 l-k-1}^{-}, \gamma \bar{\tau}_{2 l-k-1}^{+}\right)_{k}
$$

pour des constantes $\gamma \in \mathbb{C}^{*}$ et $l \in \mathbb{Z} / p \mathbb{Z}$.

On parlera d'anti-symétrie involutive anti-holomorphe lorsque cette transformation est une involution, i.e. lorsque $\gamma$ est réel.

Toute courbe lisse analytique réelle passant par $0 \in \mathbb{C}$ est conformément redressable sur l'axe réel. La coordonnée conforme est loin d'être unique puisqu'on peut la composer par n'importe quel élément de $\operatorname{Diff}(\mathbb{R}, 0)$. Cependant, cette liberté est insuffisante à redresser simultanément deux telles courbes transverses, disons sur deux droites réelles de même angle. Une obstruction, dégagée en 1917 par Pfeiffer, est la suivante. Considérons les involutions de Schwarz associées à ces deux courbes et notons $f$ leur composée. Alors $f(z)=e^{2 i \pi \alpha} z+\cdots \in \operatorname{Diff}(\mathbb{C}, 0)$ où $\frac{\alpha}{2}$ est l'angle formé entre les deux courbes. Une condition nécessaire et suffisante au redressement simultané des courbes est la linéarisabilité de $f$. C'est à cette occasion que Pfeiffer construit le premier exemple de difféomorphisme $f$ formellement mais non analytiquement linéarisable.

Si l'on considère maintenant deux courbes lisses accusant un contact d'ordre $p \in \mathbb{N}^{*}$, alors la même construction, étudiée par Nakai, nous donne comme invariant un difféomorphisme $f$ tangent à l'identité à l'ordre $p$ qui, par construction, est conjugué à son inverse par une involution antiholomorphe.

En ce qui nous concerne, nous sommes surtout motivés par le fait qu'une telle symétrie permet de reconnaître les cols résonants ou les nœud-cols 
qui admettent une équation réelle dans un bon système de coordonnées holomorphes, comme nous le verrons plus loin. On trouve dans [152] (voir aussi [221]) le

Théorème 3.33 (Nakai). Sont équivalents :

- il existe une involution anti-holomorphe $\sigma$ fixant $0 \in \mathbb{C}$ et conjugant $f \grave{a} f^{\circ(-1)}$,

- le chapelet admet une anti-symétrie involutive anti-holomorphe.

Dans ce cas, $\lambda \in \mathbb{R}$.

\subsection{Classification topologique}

Pour une raison d'indice, l'ordre de contact $p$ d'un élément $f(z)=z+$ $z^{p+1}+\cdots \in \operatorname{Diff}(\mathbb{C}, 0)$ à l'identité est un invariant topologique. En fait, A.A. Shcherbakov et C. Camacho ont indépendamment montré dans [27, 204] que c'est le seul invariant pour les germes tangents à l'identité :

Théorème 3.34 (Camacho,Shcherbakov). Soit $f(z)=z+z^{p+1}+\cdots \in$ $\operatorname{Diff}(\mathbb{C}, 0)$. Alors il existe un homéomorphisme $\Psi: U \rightarrow \mathbb{C}$ envoyant un voisinage ouvert $U$ de 0 sur un voisinage de 0 dans $\mathbb{C}$ et conjugant la dynamique de $f$ à celle de $f_{0}(z)=z /\left(1-z^{p}\right)^{1 / p}: \Psi \circ f=f_{0} \circ \Psi$.

Démonstration. On reprend l'idée déjà utilisée dans la seconde preuve du Théorème 3.3 et dans la section 3.8 : on construit une conjugaison topologique des chapelets de sphères correspondants qui se relève ensuite par une conjugaison des dynamiques. Il suffit pour cela de construire des homéomorphismes

$$
\Psi_{k}^{ \pm}: \overline{\mathbb{C}} \rightarrow \overline{\mathbb{C}} ;\left\{\begin{array}{c}
0 \mapsto 0 \\
\infty \mapsto \infty
\end{array}\right.
$$

préservant l'orientation et satisfaisant les conditions de compatibilité suivantes avec les recollements :

$$
\begin{cases}\Psi_{k}^{+}=\Psi_{k}^{-} \circ \varphi_{k}^{0} & \text { au voisinage de } 0 \\ \Psi_{k}^{-}=\Psi_{k+1}^{+} \circ \varphi_{k}^{\infty} & \text { au voisinage de } \infty\end{cases}
$$

Si l'on fixe $\Psi_{k}^{-}=$identité, $k \in \mathbb{Z} / p \mathbb{Z}$, les conditions précédentes deviennent

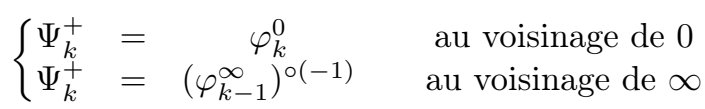

On peut facilement construire, en utilisant des résultats classiques de Whitney, de tels difféomorphismes réels $C^{\infty}$ en lissant les germes $\varphi_{k}^{0}$ et $\left(\varphi_{k-1}^{\infty}\right)^{\circ(-1)}$ vers l'identité sur une couronne. Une façon plus simple pour nous est d'utiliser le Théorème 3.3 : si l'on a préalablement choisi les coordonnées linéaires suffisamment génériques sur les sphères $S_{k}^{ \pm}$, alors 
ces germes sont hyperboliques et sont les flots au temps 1 de champs de vecteurs holomorphes $X_{k}^{0}$ et $X_{k}^{\infty}$ respectivement. À l'aide d'une fonction cloche dépendant du module $|\tau|$, on construit un champ de vecteur $X_{k}$ sur $\overline{\mathbb{C}}$ qui est $C^{\infty}$ sur $\overline{\mathbb{C}}$, identiquement nul sur un anneau $r<|\tau|<R$ et qui coincide avec $X_{k}^{0}\left(\operatorname{resp} . X_{k}^{\infty}\right)$ près de 0 (resp. $\left.\infty\right)$. Alors $\Psi_{k}^{+}:=\exp \left(X_{k}\right)$ convient. Le relèvement de l'homéomorphisme $\Psi$ ainsi construit en un homéomorphisme des atlas (et par suite de dynamiques au voisinage de $z=0$ ) est un jeu d'écriture.

Notons que la conjugaison construite est de classe $C^{\infty}$ en dehors de l'origine.

Proposition 3.35 ([139]). Soit $\Psi:(\mathbb{C}, 0) \rightarrow(\mathbb{C}, 0)$ est un germe de difféomorphisme réel de classe $C^{1}$ tangent à l'identité conjugant deux germes de difféomorphisme holomorphes $f, g \in \operatorname{Diff}(\mathbb{C}, 0)$ de la forme $z+z^{2}+\cdots$. Alors $\Psi \in \operatorname{Diff}(\mathbb{C}, 0)$.

Démonstration. On relève $\Psi(z)$ en une conjugaison $\tilde{\Psi}(w)$ des atlas trivialisant. Sur chaque pétale, $\tilde{\Psi}$ commute à la translation $w \mapsto w+1$. Par ailleurs, la différentielle de $\tilde{\Psi}(w)$ tend vers l'identité lorsque $w \rightarrow \infty$ (quelle que soit la direction). En effet, les coordonnées trivialisantes sont asymptotiques à $\psi_{1, \lambda}=-\frac{1}{z}+\frac{\lambda}{2 i \pi} \log (z)$ et cette dernière est la composée de $z \mapsto-1 z$ avec $w \mapsto w-\frac{\lambda}{2 i \pi} \log (w)+$ constante; observons alors que $\Psi$ devient continûment différentiable à l'infini après conjugaison par la première et que la seconde est tangente à l'identité à l'infini. Maintenant, la différentielle de $\tilde{\Psi}$ est constante le long des orbites de la translation et donc identiquement égale à sa limite à l'infini : $\tilde{\Psi}$ est une translation sur chaque pétale, donc holomorphe.

Notons que la transformation linéaire réelle $x+i y \mapsto x+\tau y$, avec $\operatorname{Im}(\tau)>$ 0 , commute à la translation $w \mapsto w+1$. Dans la variable $z=1 /(x+i y)$, on déduit un germe d'homéomorphisme $\Psi$ qui commute à $z /(1-z)$ et qui est analytique en dehors de 0 , mais pas différentiable en 0 .

\subsection{Classification analytique des sous-groupes résolubles de $\operatorname{Diff}(\mathbb{C}, 0)$}

On a vu dans la section 3.1 des conditions suffisantes sur $a \in \mathbb{C}^{*}$ pour que tout germe $f(z)=a z+\cdots \in \operatorname{Diff}(\mathbb{C}, 0)$ soit analytiquement linéarisable, à savoir $|a| \neq 1$ ou $a \in \mathcal{B} \subset \mathbb{S}^{1}$ est un nombre de Brjuno. Cependant, pour un nombre $a \in \mathbb{S}^{1} \backslash \mathcal{B}$ non périodique, on ne sait pas décrire l'espace des classes de conjuguaison analytique des germes $f(z)=a z+\cdots \in \operatorname{Diff}(\mathbb{C}, 0)$ (bien qu'il soit non dénombrable d'après Yoccoz, Théorème 3.7). Par contre, lorsque $a$ est périodique, la classification analytique est bien comprise. 
De la même manière, seuls les sous-groupes formellement linéarisables de $\operatorname{Diff}(\mathbb{C}, 0)$ restent difficile à décrire aujourd'hui. Un sous-groupe non formellement linéarisable, lui, contient toujours des éléments tangents à l'identité (voir Corollaire 2.23) et leur présence va nous permettre de comprendre complètement leur dynamique.

Théorème 3.36 (Cerveau-Moussu). Soit $G$ un sous-groupe de Diff( $(\mathbb{C}, 0)$ et soit $G_{1}$ le sous-groupe des éléments tangents à l'identité. Si $G_{1}$ est de rang $\geq 2$, alors toute conjugaison formelle $\hat{\varphi} \in \widehat{\operatorname{Diff}}(\mathbb{C}, 0)$ avec un autre sous-groupe $\tilde{G} \subset \operatorname{Diff}(\mathbb{C}, 0)$ est en fait analytique :

$$
\hat{\varphi}_{*} G=\tilde{G} \quad \Rightarrow \quad \hat{\varphi} \in \operatorname{Diff}(\mathbb{C}, 0) .
$$

Démonstration dans le cas où $G$ est résoluble. D'après le Théorème 2.22, $G_{1}$ est abélien et contenu dans le groupe à 1 paramètre formel $\exp (t X)$ d'un champ de vecteur formel. Le Corollaire 3.26 nous dit que $X$ est convergent (sinon, $G_{1}$ serait de rang 1 ). Le même argument s'applique à $\tilde{G}$, et $\tilde{G}_{1}$ est plongeable dans le groupe à 1 paramètre d'un champ $\tilde{X}$ convergent. Par unicité du logarithme formel (voir Corollaire 2.18), $\hat{\varphi}$ conjugue $X$ et $\tilde{X}$ et la Proposition 2.3 nous donne une conjugaison convergente $\varphi$; par la Proposition $2.17, \hat{\varphi}=\tilde{f} \circ \varphi$ où $\tilde{f}$ est dans le groupe à 1 paramètre $\exp (t \tilde{X})$ et donc convergent.

Remarque 3.37. Lorsque $G$ n'est pas résoluble, on utilise des arguments de sommabilité. Si $\hat{\varphi}$ conjugue deux difféomorphismes tangents à l'identité $f(z)=z+z^{p+1}+\cdots$ et $\tilde{f}(z)=z+z^{p+1}+\cdots$, alors $\hat{\varphi}$ est p-sommable d'après [139] (voir [9] pour la notion de p-sommabilité). Par ailleurs, $G_{1}$ contient des éléments tangents à l'identité d'ordre arbitrairement grand d'après le Théorème 2.22 : ainsi, $\hat{\varphi}$ est $p$-sommable pour différents $p \in \mathbb{N}^{*}$. D'après [178] (voir aussi [9], Theorem 3, p.30), ceci implique que $\hat{\varphi}$ est convergente. On trouvera d'autres preuves dans [74] et [219].

Corollaire 3.38. Soit $G$ un sous-groupe résoluble de $\operatorname{Diff}(\mathbb{C}, 0)$. On suppose que le sous-groupe $G_{1}$ des éléments tangents à l'identité est de rang $\geq 2$. Alors $G$ est analytiquement conjugué à un sous-groupe d'un des groupes de Lie suivants :

- $\mathbb{E}_{p, \lambda}=\left\{f(z)=a \exp \left(t X_{p, \lambda}\right) ; a^{p}=1, t \in \mathbb{C}\right\}, \lambda \in \mathbb{C}, p \in \mathbb{N}^{*}$,

- $\mathbb{A}_{p}=\left\{f(z)=a z /\left(1-b z^{p}\right)^{1 / p} ; a \in \mathbb{C}^{*}\right.$ et $\left.b \in \mathbb{C}\right\}, p \in \mathbb{N}^{*}$.

Démonstration. On applique le Théorème 3.36 à la normalisante formelle $\hat{\varphi}$ construite dans le Théorème 2.22. Rappelons que $G$ n'est pas formellement linéarisable d'après le Corollaire 2.23.

Proposition 3.39. Soit $G$ un sous-groupe de $\operatorname{Diff}(\mathbb{C}, 0)$. On suppose que le sous-groupe $G_{1}$ des éléments tangents à l'identité est de rang 1 . Alors on est dans l'une des situations suivantes: 
1. G est abélien et formellement conjugué au groupe engendré par

$$
f=\exp \left(X_{k q, \lambda}\right) \quad \text { et } \quad g=e^{\frac{2 i \pi}{q}} \exp \left(\frac{n}{q} X_{k q, \lambda}\right)
$$

où $\lambda \in \mathbb{C}, q, k \in \mathbb{N}^{*}$ et $n \in \mathbb{Z} ;$ comme groupe abstrait, $G$ admet la présentation

$$
G=<f, g ; f^{\circ n}=g^{\circ q}, f \circ g=g \circ f>\quad \text { et } \quad G_{1}=<f>.
$$

2. G n'est pas abélien et est formellement conjugué au groupe engendré par

$$
f(z)=z /\left(1-z^{p}\right)^{1 / p} \quad \text { et } \quad g(z)=e^{\frac{2 i \pi}{2 q}} z
$$

où $p, q \in \mathbb{N}^{*}$ avec $p=k q, k$ impair (ici, $\lambda=0$ ); comme groupe abstrait, $G$ admet la présentation

$$
G=<f, g ; g^{\circ 2 q}=\text { identité, } g \circ f \circ g^{\circ(-1)}=f^{\circ(-1)}>\text { et } G_{1}=<f>\text {. }
$$

Notons que le sous-groupe $\left\langle f, g^{\circ 2}>\right.$ est abélien d'indice 2 dans $G$.

Démonstration. Soit $f$ un générateur de $G_{1}$ : dans une bonne coordonnée formelle, $f=\exp \left(X_{p, \lambda}\right)$ pour des $p \in \mathbb{N}^{*}$ et $\lambda \in \mathbb{C}$ d'après la Proposition 2.16 .

Si $G$ est abélien, alors il est contenu dans le centralisateur formel de $f$ et, d'après la Proposition 2.17, tout élément $g \in G$ est de la forme $g=a \exp \left(t X_{p, \lambda}\right)$ avec $a^{p}=1$ et $t \in \mathbb{C}$. Soit $q$ l'ordre du sous-groupe de $\mathbb{C}^{*}$ engendré par les parties linéaires $a$ des éléments de $G$ et choisissons $g \in G$ dont la partie linéaire est $a=e^{\frac{2 i \pi}{q}}$. Alors $f$ et $g$ engendrent $G:$ si $h \in G$, alors sa partie linéaire coïncide avec celle de $g^{\text {or }}$ pour un $0 \leq r \leq q$ convenable et $h \circ g^{\circ(-r)} \in G_{1}$ est une itérée de $f: h=f^{\circ s} \circ g^{\circ r}$. Puisque $a^{p}=1$, on peut écrire $p=k q$; enfin, $g^{\circ q}=\exp \left(q t X_{p, \lambda}\right) \in G_{1}$ et donc $q t=n \in \mathbb{Z}$.

Si $G$ n'est pas abélien, puisque $G_{1}$ est un sous-groupe normal, pour tout $g \in G$ il vient $g \circ f \circ g^{\circ(-1)}=f$ ou $f^{\circ(-1)}$ (un des deux générateurs possibles de $G_{1}$ ). La deuxième possibilité se produit pour au moins un élément $g \in G$ car sinon tous les éléments commuteraient à $f$ et $G$ serait abélien. En particulier, $f$ est conjuguée à $f^{\circ(-1)}$ et $\lambda=0$ d'après la Remarque 2.20. En utilisant le développement $f(z)=z+z^{p+1}+\cdots$, on remarque que la partie linéaire $a$ d'un élément $g \in G$ satisfait $a^{p}=1$ ou -1 selon que $g$ commute ou anti-commute à $f$, et donc $a^{2 p}=1$. Le sous-groupe de $\mathbb{C}^{*}$ engendré par les parties linéaires $a$ des éléments de $G$ est donc d'ordre fini $2 q$ où $p=k q$ avec $k$ impair; en effet, si l'ordre de ce groupe divisait $p$, tous les éléments de $G$ commuteraient à $f$, ce que l'on a exclu. Soit $g \in G$ dont la partie linéaire est $a=e^{\frac{2 i \pi}{2 q}}$. Remarquons que le difféomorphisme linéaire $g_{0}(z)=a z$ anti-commute à $X_{p, 0}$ et donc à $f$; par suite, $g_{0}^{\circ(-1)} \circ g$ 
est tangent à l'identité et commute à $f$, de sorte que $g=a \cdot \exp \left(t X_{p, 0}\right)$ pour un $t \in \mathbb{C}$. Comme dans le cas abélien, $f$ et $g$ engendrent $G$. Finalement, si $\varphi=\exp \left(\frac{t}{2} X_{p, 0}\right)$, on a $\varphi \circ f \circ \varphi^{\circ(-1)}=f$ et $\varphi \circ g \circ \varphi^{\circ(-1)}=a z$ devient linéaire.

Remarque 3.40. Dans le cas abélien, le groupe $G$ admet aussi la présentation suivante. Notons d le diviseur commun de $n$ et $q: n=d \tilde{n}, q=d \tilde{q}$ et par Bézout il existe $a, b \in \mathbb{N}$ tels que a $\tilde{n}-b \tilde{q}=1$. Considérons maintenant les éléments

$$
\tilde{f}=f^{\circ(-b)} \circ g^{\circ a}=e^{2 i \pi \frac{a}{q}} \exp \left(\frac{1}{\tilde{q}} X_{k q, \lambda}\right) \quad \text { et } \quad \tilde{g}=f^{\circ(-\tilde{n})} \circ g^{\circ \tilde{q}}=e^{\frac{2 i \pi}{d}} z .
$$

Alors $\tilde{f}^{\circ \tilde{q}} \circ \tilde{g}^{\circ(-a)}=f$ et $\tilde{f}^{\circ \tilde{n}}=g$. Par ailleurs, $\tilde{g}$ engendre le sous-groupe de torsion (d'ordre d) et $\tilde{f}$ est une racine d'ordre $\tilde{q}$ de $f$ dans $G$. Notons que $G$ est cyclique (i.e. de rang 1 ) dès qu'il ne contient pas de torsion.

Pour chacun des groupes $G$ considérés dans la Proposition 3.39, l'ensemble des classes de conjugaison analytique des sous-goupes de Diff $(\mathbb{C}, 0)$ formellement conjugués à $G$ est non dénombrable. Plus précisément, considérons pour commencer le cas abélien : $G$ est engendré par

$$
f=\exp \left(X_{k q, \lambda}\right) \quad \text { et } \quad g=e^{\frac{2 i \pi}{q}} \cdot \exp \left(\frac{n}{q} X_{k q, \lambda}\right) .
$$

Le quotient d'un voisinage épointé $U^{*}$ de 0 par la dynamique de $G$ s'identifie au quotient du chapelet de sphères associé à $f$ par le difféomorphisme $\bar{g}$ induit par $g:$ d'après le Théorème $3.25, \bar{g}$ est une permutation cyclique d'ordre $q$ des sphères (respectant l'orientation et la polarité). Le quotient final est donc un chapelet polarisé et orienté à $2 k$ sphères dont la partie linéaire est $e^{2 i \pi \frac{\lambda}{q}}$. C'est un jeu d'écritures de vérifier que les classes analytiques des sous-groupes de $\operatorname{Diff}(\mathbb{C}, 0)$ formellement conjugués à $G$ sont décrites par ce type de chapelets. Nous renvoyons à [3] pour un énoncé précis et plus de détails.

Dans le cas non abélien, $G$ est engendré par

$$
f(z)=z /\left(1-z^{p}\right)^{1 / p} \quad \text { et } \quad g(z)=e^{\frac{2 i \pi}{2 q}} z, \quad p=k q, k \text { impair. }
$$

Comme au-dessus, $g$ induit un difféomorphisme $\bar{g}$ périodique d'ordre $2 q$ sur le chapelet associé à $f$ qui renverse la polarité mais préserve l'orientation. Le quotient est un chapelet orienté à $k$ sphères ( $k$ impair) dont la partie linéaire est triviale. De nouveau, se donner un tel chapelet, c'est se donner une classe analytique de sous-groupe formellement conjugué à $G$. 


\subsection{Remarques sur les sous-groupes non ré- solubles de $\operatorname{Diff}(\mathbb{C}, 0)$}

Une conséquence du Théorème 3.36 est que les Proposition 2.24 et Théorème 2.25 restent vrais en analytique. Nous reprenons les notations de la section 2.5 : étant donné un sous-groupe non résoluble $G \subset \operatorname{Diff}(\mathbb{C}, 0)$, on note

$$
G_{k}=\left\{c \in \mathbb{C} ; \exists g(z)=z+c z^{k+1}+\cdots \in G\right\} \quad \text { pour } \quad k \in \mathbb{N}^{*}
$$

et

$$
K=\left\{k \in \mathbb{N}^{*} ; G_{k} \neq\{0\}\right\} .
$$

Corollaire 3.41 ([119]). Pour $d \in \mathbb{N}^{*}$, sont équivalents :

$-\operatorname{pgcd}(K)=d$,

- le centralisateur formel de $G$ est d'ordre d,

- le centralisateur analytique de $G$ est d'ordre $d$.

Dans ce cas, il existe une coordonnée analytique $z$ dans laquelle tous les éléments de $G$ sont de la forme $g(z)=z \tilde{g}\left(z^{d}\right)$.

Démonstration. Compte tenu de la Proposition 2.24, il suffit de montrer que le centralisateur formel est analytique, c'est-à-dire que tout élément $\hat{\varphi} \in \widehat{\operatorname{Diff}}(\mathbb{C}, 0)$ commutant à tous les éléments de $G$ est en fait analytique. Mais c'est une conséquence directe du Théorème 3.36 .

Corollaire 3.42 ([119]). Sont équivalents :

- $G_{k}$ est contenu dans une droite réelle pour tout $k \in K$,

- $G_{k}$ est contenu dans une droite réelle pour $k>>0$,

- $G$ laisse invariante une courbe analytique lisse réelle passant par 0 ,

- $G$ est analytiquement conjugué à un sous-groupe de $\operatorname{Diff}(\mathbb{R}, 0)$.

Dans ce cas, il existe une coordonnée analytique $z$ dans laquelle tous les éléments de $G$ sont de la forme $g(z)=z \tilde{g}\left(z^{d}\right), \tilde{g} \in \mathbb{R}[[x]]$, où $d=\operatorname{pgcd}(K)$.

Démonstration. Compte tenu du Théorème 2.25, il suffit de montrer que si $G$ est formellement conjugué à un sous-groupe de $\widehat{\operatorname{Diff}}(\mathbb{R}, 0)$, alors il est aussi analytiquement conjugué à un sous-groupe de $\operatorname{Diff}(\mathbb{R}, 0)$. Supposons donc que $\hat{\varphi}_{*} G \subset \widehat{\operatorname{Diff}}(\mathbb{R}, 0)$. Alors l'involution anti-holomorphe $\sigma_{0}(z)=\bar{z}$ commute à tous les éléments de $\hat{\varphi}_{*} G$; en la tirant en arrière par $\hat{\varphi}$, on récupère une involution formelle anti-complexe $\hat{\sigma}=\hat{\varphi}^{\circ(-1)} \circ \sigma_{0} \circ \hat{\varphi}$ qui commute à tous les éléments de $G$. Par ailleurs, l'involution $\sigma_{0}$ envoie $G$ sur un autre sous-groupe $\tilde{G}=\left(\sigma_{0}\right)_{*} G \subset \operatorname{Diff}(\mathbb{C}, 0)$; par suite, le difféomorphisme formel $\hat{\psi}=\sigma_{0} \circ \hat{\sigma}$ conjugue $G$ à $\tilde{G}$ et converge par le Théorème 3.36. Ainsi, l'involution anti-complexe $\hat{\sigma}=\sigma_{0} \circ \hat{\psi}$ converge aussi et se redresse sur l'involution standart $\sigma_{0}$ par un difféomorphisme analytique $\varphi$. Alors $\varphi_{*} G$ commute à $\sigma_{0}$ et est réel. 


\section{Chapitre 4}

\section{Dynamique des sous-groupes de type fini de $\operatorname{Diff}(\mathbb{C}, 0)$}

On s'intéresse ici à l'étude qualitative de la dynamique locale induite par un sous-groupe $G \subset \operatorname{Diff}(\mathbb{C}, 0)$ de type fini de $\operatorname{Diff}(\mathbb{C}, 0)$. On se donne un système $f_{1}, \ldots, f_{k}$ de générateurs d'un sous-groupe $G \subset \operatorname{Diff}(\mathbb{C}, 0)$ et $U$ un voisinage ouvert connexe de $0 \in \mathbb{C}$ suffisamment petit de sorte que les $f_{i}$ et leurs inverses soient tous bien définis et injectifs sur $U$. On s'intéresse alors à la dynamique du pseudo-groupe engendré par les $f_{i}$ sur $U$ (voir la définition générale dans la Section 4.4). On appelle orbite d'un point $z_{0}$ et on note $\operatorname{Orb}\left(z_{0}\right)$ l'ensemble des points $z \in U$ accessibles par une suite finie $\left(z_{0}, z_{1}, \ldots, z_{n}=z\right)$ de points de $U$ obtenus en itérant indifféremment les générateurs :

$$
z_{j}=f_{i_{j}}^{\circ \varepsilon_{j}}\left(z_{j-1}\right) \quad \text { avec } \quad i_{j} \in\{1, \ldots, k\}, \varepsilon_{j} \in\{ \pm 1\}
$$

pour $j=1, \ldots, n$. On dira que la suite $\left(z_{0}, \ldots, z_{n}\right)$ est la trajectoire associée au mot $f_{i_{n}}^{\circ \varepsilon_{n}} \circ \cdots \circ f_{i_{1}}^{\circ \varepsilon_{1}}$. Une orbite sera dite complète si l'on peut itérer indifféremment les générateurs le long de l'orbite sans jamais sortir de $U$; en général, la seule orbite complète est réalisée par le point fixe $\{0\}$. Les orbites sont dénombrables, mais elles peuvent être denses ou discrètes, voire finies. La dynamique ainsi définie dépend à la fois du choix des générateurs $f_{1}, \ldots, f_{k} \in G$ et de la taille de $U$. Mais comme nous le verrons, la plupart des propriétés qualitative de la dynamique ne dépendront en fait que de $G$, pourvu que $U$ soit suffisamment petit. Un mot $f_{i_{n}}^{\circ \varepsilon_{n}} \circ \cdots \circ f_{i_{1}}^{\circ \varepsilon_{1}}$ définit à la fois un élément de $\hat{f} \in \operatorname{Diff}(\mathbb{C}, 0)$ et une dynamique $f: V \rightarrow U$ sur l'ouvert $V$ des points de $U$ pour lesquels la trajectoire associée est bien 
définie; il arrive que $V$ ne soit pas connexe et que $f$ ne coincide pas avec le prolongement analytique de $\hat{f}$ sur les composantes connexes de $V$ ne contenant pas $0 \in \mathbb{C}$ (voir Remarque 4.16). C'est un phénomène typique des pseudo-groupes. Pour cette raison, la notion de relation sera à prendre avec beaucoup de précaution.

Nous allons décrire successivement les dynamiques résolubles puis non résolubles.

\subsection{Dynamiques résolubles}

Nous allons les classer en fonction du type de l'algèbre de Lie formelle associée à $G$ (voir Théorème 2.22) à savoir les dynamiques de type linéaire, euclidienne ou affine. Dans chacun des cas se rajoutent des dynamiques exotiques (ou exceptionnelles), à savoir celles dont la tranformation normalisante $\hat{\varphi}$ construite dans le Théorème 2.22 diverge.

\subsubsection{Type (Fin) : dynamiques finies}

Si $G \subset \operatorname{Diff}(\mathbb{C}, 0)$ est fini, alors $G$ est analytiquement linéarisable (voir Proposition 3.2) :

$$
G=\left\{f(z)=e^{2 i \pi k / p} z ; k \in \mathbb{Z} / p \mathbb{Z}\right\}
$$

pour un $p \in \mathbb{N}^{*}$. Le pseudo-groupe induit par $G$ est alors la trace sur $U$ d'un groupe fini de rotations. La coordonnée $z$ est bien loin d'être unique. Ce type d'holonomie est caractéristique des singularités de feuilletages admettant une intégrale première holomorphe ou méromorphe (voir [140]). Une intégrale première de ce pseudo-groupe est par exemple donnée par $H(z)=z^{p}$.

\subsubsection{Type (Lin) : dynamiques linéaires}

Ici, $G$ est, dans une bonne coordonnée analytique, un sous-groupe du groupe linéaire :

$$
\mathbb{L}=\left\{f(z)=a z ; a \in \mathbb{C}^{*}\right\}
$$

où l'on suppose de plus que $G$ contient un élément d'ordre infini pour ne pas retomber dans la situation précédente (on suppose ici $G$ de type fini). Le pseudo-groupe $G$ est alors la trace sur $U$ d'un groupe de transformations linéaires. La coordonnée linéarisante $z$ est unique à composition près par une transformation linéaire. Ce type de dynamiques est caractéristique de l'holonomie d'un feuilletage défini par une 1-forme méromorphe fermée au voisinage d'un pôle simple. En fait, la 1-forme $\omega=\frac{d z}{z}$ est invariante par $G: f^{*} \omega=\omega$ pour tout $f \in \mathbb{L}$. 
Proposition 4.1. Si un sous-groupe $G \subset \operatorname{Diff}(\mathbb{C}, 0)$ laisse invariant un germe de 1-forme méromorphe $\omega$ ayant un pôle simple en 0 , alors $G$ est analytiquement linéarisable.

Démonstration. D'après la Proposition 2.3, la 1-forme $\omega$ est analytiquement équivalente à sa partie principale $\alpha \frac{d z}{z}$. Maintenant, dire que $f \in G$ laisse invariante cette dernière nous conduit à l'équation différentielle $\frac{d f}{f}=$ $\frac{d z}{z}$ laquelle nous donne, après intégration, $f=a z, a \in \mathbb{C}^{*}$.

Proposition 4.2. Si $\tilde{G} \subset \operatorname{Diff}(\mathbb{C}, 0)$ est conjugué à $G$ par un homéomorphisme $\Psi:(\mathbb{C}, 0) \rightarrow(\mathbb{C}, 0)$ préservant l'orientation, alors $\tilde{G}$ est analytiquement linéarisable.

Démonstration. Il suffit de montrer qu'un élément $\tilde{f} \in \tilde{G}$ d'ordre infini est linéarisable et d'appliquer la Proposition 2.17. Pour cela, on considère un tel élément $f \in G$; quitte à remplacer $f$ par $f^{\circ(-1)}$, on peut supposer $\left|f^{\prime}(0)\right| \leq 1$. Alors $f(\mathbb{D}) \subset \mathbb{D}$ pour tout disque suffisamment petit centré en 0 et son image $\tilde{f}$ va préserver $\Psi(\mathbb{D})$. On conclut avec la Proposition suivante.

Proposition 4.3 (Théorème du domaine invariant). Si $f=a z+\cdots \in$ $\operatorname{Diff}(\mathbb{C}, 0)$ et si $U \subset \mathbb{C}$ est un voisinage ouvert de 0 sur lequel $f$ est bien définie et $f(U) \subset U$, alors $f$ est linéarisable.

Démonstration. D'après le Théorème d'Uniformisation des surfaces de Riemann, ou bien $U=\mathbb{C}$ (auquel cas $f$ doit être affine et donc linéarisable), ou bien il existe un revêtement conforme $\varphi: \mathbb{D} \rightarrow U$ par le disque unité sur lequel $f$ se relève en une application $\tilde{f}: \mathbb{D} \rightarrow \mathbb{D}$ fixant 0 ; en particulier, $\varphi$ conjugue $\tilde{f}$ à $f$ en 0 . Dans ce dernier cas, $\tilde{f}$ et ses itérées positives $\tilde{f}^{\circ n}$ sont uniformément bornées sur $\mathbb{D}$. D'après la Formule de Cauchy, les dérivées sont elles aussi bornées en 0 , ce qui implique que $|a| \leq 1$. Lorsque $|a|<1$, on applique juste le Théorème 3.3. Maintenant, si $|a|=1$, le Lemme de Schwarz nous dit que $\tilde{f}$ est linéaire.

Remarque 4.4. Étant donné un automorphisme linéaire contractant $f$ : $z \mapsto a z,|a|<1$, le flot associé $f^{\circ t}: z \mapsto e^{2 i \pi \alpha t} z, a=e^{2 i \pi \alpha}$, dépend $d u$ logarithme $\alpha$ que l'on a choisi pour a. A fortiori, $f$ n'a pas de trajectoire réelle canonique, excepté le cas $a \in \mathbb{R}$ où, pour $\alpha$ imaginaire pur, les trajectoires sont analytiques réelles en 0 .

Remarque 4.5. Dans la Proposition 4.2, les groupes $G$ et $\tilde{G}$ ne sont pas analytiquement conjugués en général. Par exemple, on peut construire une conjugaison topologique entre deux contractions linéaires $f(z)=a z$ et $g(z)=b z$ de la manière suivante. Relevons les deux dynamiques par l'exponentielle $z=e^{2 i \pi w}$ :

$$
\tilde{f}(w)=w+\alpha \quad \text { et } \quad \tilde{g}(z)=w+\beta, \quad \alpha, \beta \in \mathbb{H}
$$


où $\mathbb{H}=\{\operatorname{Im}(w)>0\}$ est le demi plan supérieur. On peut alors trouver une transformation linéaire réelle $\tilde{\Psi}(x+i y)=x+i y+\lambda i y$ commutant à la translation $w \mapsto w+1$ et conjugant $\tilde{f} \grave{a} \tilde{g}$. Elle définit, par passage au quotient, un difféomorphisme analytique réel

$$
\Psi: \mathbb{C}^{*} \rightarrow \mathbb{C}^{*} ; z \mapsto|z|^{\lambda} z
$$

conjugant $f \grave{a} g$. On vérifie que $\operatorname{Im}(\lambda)>-1$ et donc que $\Psi$ se prolonge continûment en $z=0$.

Par contre, deux rotations $f(z)=e^{2 i \pi \alpha} z$ et $g(z)=e^{2 i \pi \beta} z, \alpha, \beta \in \mathbb{R}$, qui sont topologiquement conjuguées sont automatiquement analytiquement conjuguées. En effet, le fait de ne pas contracter ni dilater est une propriété topologique; par ailleurs, le développement de $\alpha$ en fraction continue se lit topologiquement sur la dynamique de $f$ en restriction à n'importe quel cercle invariant.

Lemme 4.6. Soient $\Gamma=<\alpha, \beta, \gamma>$ un sous-groupe additif de $\mathbb{R}^{2}$ avec $\alpha, \beta, \gamma \neq(0,0)$ non tous colinéaires et $\Delta_{\alpha}=\operatorname{det}(\beta, \gamma), \Delta_{\beta}=\operatorname{det}(\gamma, \alpha)$ et $\Delta_{\gamma}=\operatorname{det}(\alpha, \beta)$ :

$$
\left\{\begin{array}{l}
\alpha=\left(\alpha_{1}, \alpha_{2}\right) \\
\beta=\left(\beta_{1}, \beta_{2}\right) \\
\gamma=\left(\gamma_{1}, \gamma_{2}\right)
\end{array} \quad\left(\begin{array}{l}
\alpha_{1} \\
\beta_{1} \\
\gamma_{1}
\end{array}\right) \wedge\left(\begin{array}{c}
\alpha_{2} \\
\beta_{2} \\
\gamma_{2}
\end{array}\right)=\left(\begin{array}{c}
\Delta_{\alpha} \\
\Delta_{\beta} \\
\Delta_{\gamma}
\end{array}\right) \neq 0 .\right.
$$

On note $\nu$ le nombre de relations entre $\Delta_{\alpha}, \Delta_{\beta}, \Delta_{\gamma} \in \mathbb{R}$ sur $\mathbb{Z}: \nu$ est le rang du sous-module $R$ des $(m, n, p) \in \mathbb{Z}^{3}$ vérifiant $m \Delta_{\alpha}+n \Delta_{\beta}+p \Delta_{\gamma}=0$. Alors on a la trichotomie suivante:

$-\nu=2$ et $\Gamma$ est discret,

$-\nu=1$ et $\bar{\Gamma}=\mathbb{Z} u+\mathbb{R} v$ avec $\operatorname{det}(u, v) \neq 0$,

$-\nu=0$ et $\Gamma$ est dense.

Démonstration. Les sous-groupes de $\mathbb{Z}^{3}$ sont de rang $0,1,2$ ou 3 ; si $\nu=3$, alors $\Delta_{\alpha}=\Delta_{\beta}=\Delta_{\gamma}=0$ ce que l'on a exclu. Supposons pour la suite $\Delta_{\gamma} \neq 0:\left\langle\alpha, \beta>\right.$ est un sous-groupe discret de $\mathbb{R}^{2}$. Dans $\mathbb{R}^{2} /\langle\alpha, \beta\rangle, \gamma$ est repéré par ses nombres de rotation respectifs $r_{\alpha}=-\frac{\Delta_{\alpha}}{\Delta_{\gamma}}$ et $r_{\beta}=-\frac{\Delta_{\beta}}{\Delta_{\gamma}}$ sur $\alpha$ et $\beta$. En particulier, $\nu$ compte le nombre de relations liant $r_{\alpha}$ et $r_{\beta}$ à $\mathbb{Z}$, d'où le résultat.

Corollaire 4.7. Soit $G$ un sous-groupe linéaire de type fini de $\operatorname{Diff}(\mathbb{C}, 0)$ :

$$
G=<z \mapsto a_{1} z, \ldots, z \mapsto a_{k} z>, \quad a_{1}, \ldots, a_{k} \in \mathbb{C}^{*}
$$

agissant sur un disque $U=\{|z|<r\}$. Alors on est dans l'une des situations suivantes :

1. $G$ est fini cyclique : $G=<z \mapsto a z>, a^{p}=1$. 
2. $G$ est discret dans $\mathbb{C}^{*}: G=<z \mapsto a z, z \mapsto b z>, a=e^{2 i \pi \alpha}$, $\alpha \in \mathbb{C} \backslash \mathbb{R}, b^{q}=1$. Le quotient de $U^{*}$ par $G$ est le tore $\mathbb{T}_{\alpha}=\mathbb{C} / \mathbb{Z}+$ $\alpha \mathbb{Z}$ et les intégrales premières les plus régulières de $G$ sont méromorphes sur $U^{*}$ : ce sont les fonctions rationnelles de $\wp_{\alpha}\left(\frac{1}{2 i \pi} \log (z)\right)$ et $\wp_{\alpha}^{\prime}\left(\frac{1}{2 i \pi} \log (z)\right)$ où $\wp_{\alpha}$ et $\wp_{\alpha}^{\prime}$ sont les fonctions de Weierstrass associées à $\mathbb{T}_{\alpha}$.

3. L'adhérence $\bar{G}$ dans $\mathbb{C}^{*}$ est de dimension 1 réelle et deux cas se présentent :

(a) $\bar{G}=<z \mapsto a z, z \mapsto e^{2 i \pi t} z ; t \in \mathbb{R}>, a=e^{2 i \pi \alpha}, \alpha \in \mathbb{C} \backslash \mathbb{R} ;$ alors l'adhérence de toute orbite dans $U^{*}$ est un cercle ou une union infinie de cercles centrés en 0.

(b) $\bar{G}=<z \mapsto e^{2 i \pi t \alpha} z, z \mapsto b z ; t \in \mathbb{R}>, \alpha \in \mathbb{C} \backslash \mathbb{R}, b^{q}=1$; alors l'adhérence de toute orbite dans $U^{*}$ est une union finie de spirales (ou de rayons).

4. $G$ est dense dans $\mathbb{C}^{*}$ et toute orbite est dense dans $U^{*}$.

Démonstration. On applique le Lemme précédent aux relèvements $w \mapsto$ $w+\alpha_{i}$ des générateurs de $G$ par l'exponentielle $w=e^{2 i \pi z}$ en tenant compte de la transformation de revêtement $w \mapsto w+1$.

Remarque 4.8. Les orbites induites par un système de générateurs d'un groupe linéaire comme dans le Corollaire 4.7 sur un disque coincident avec les restrictions des orbites induites par les mêmes générateurs sur $\mathbb{C}^{*}$ essentiellement à cause de l'abélianité : tout mot en les générateurs peut être réordonné de sorte que l'on applique les contractions avant d'appliquer les dilatations; la trajectoire correspondante termine sur le même point mais reste entièrement contenue dans le disque.

Remarque 4.9. L'ensemble des couples $(a, b) \in \mathbb{C}^{*} \times \mathbb{C}^{*}$ qui engendrent un sous-groupe dense $\left\langle a, b>\subset \mathbb{C}^{*}\right.$ est de mesure de Lebesgue totale; cependant, l'ensembles des paires qui engendrent un sous-groupe discret est dense dans $\mathbb{C}^{*} \times \mathbb{C}^{*}$.

Remarque 4.10. Dans le Corollaire 4.7, la classification présentée est bien sûr de nature topologique. Par contre, seuls les sous-groupes de rotations sont rigides au sens où toute conjugaison topologique entraine la conjugaison analytique. Les autres se déforment par l'argument de la Remarque 4.5 .

\subsubsection{Type $(\operatorname{Lin})^{\mathrm{ex}}$ : dynamiques de Pérez-Marco}

Pour démontrer le Théorème 3.7, J.-C. Yoccoz construit pour tout $\alpha \in$ $\mathbb{R} \backslash \mathcal{B}$ non rationnel des germes $f(z)=e^{2 i \pi \alpha} z+\cdots$ possédant des orbites périodiques arbitrairement proche de 0 . Ceci signifie qu'elles sont présentes dans tout voisinage de 0 et sont une obstruction dynamique à 
la linéarisation puisque $a$ n'est pas périodique. De plus, la liberté de sa construction est telle qu'il peut prescrire la dynamique de retour au voisinage de chacune de ces orbites : si $z_{0}$ est un point périodique d'ordre $q$ pour $f$, alors l'application de retour est le germe de dynamique induit par $f^{\circ q}$ au voisinage de son point fixe $z_{0}$. En particulier, à chaque orbite périodique on peut associer le multiplicateur $\left(f^{\circ q}\right)^{\prime}\left(z_{0}\right)$; en faisant varier ces multiplicateurs, on déduit un espace de modules de dimension infinie.

Cependant, R. Pérez-Marco a mis en évidence l'existence de germes non linéarisables sans aucune orbite périodique dès que $\alpha$ est très bien approché par les rationnels, à savoir les réduites $\frac{p_{n}}{q_{n}}$ de $\alpha$ (définies par (3.2), section 3.1) satisfont

$$
\left(\mathcal{B}^{\prime}\right) \quad \sum_{n \geq 1} \frac{\log \left(\log \left(q_{n}\right)\right)}{q_{n}}=+\infty .
$$

Plus précisément, toutes les orbites positives complètes du germe $f$ construit accumulent 0 . Par contre, il montre que pour $\alpha \in \mathcal{B} \backslash \mathcal{B}^{\prime}$, tout germe non linéarisable possède des orbites périodiques (arbitrairement proche de 0).

Dans une série d'articles ultérieurs, Pérez-Marco donne une description plus systématiques de ces dynamiques non linéarisables. Il montre par exemple le :

Théorème 4.11 (Pérez-Marco). Soit $f(z)=a z+\cdots \in \operatorname{Diff}(\mathbb{C}, 0)$ non linéarisable et non résonant. Alors il existe un compact $K$ (dont la taille dépend de l'ouvert $U$ de définition) invariant par $f$ et vérifiant :

- $K$ est la composante connexe contenant 0 de l'ensemble des orbites complètes $\cap_{n \in \mathbb{Z}} f^{\circ n}(U)$ dans $U$,

- $K$ est connexe et plein $(\mathbb{C}-K$ est simplement connexe) et contient 0 ,

- $K \backslash\{0\}$ a une quantité non dénombrable de composantes connexes,

- 0 est le seul point localement connexe de $K$,

- si $\mu_{K}$ désigne la mesure harmonique de $K$ à l'infini dans $\overline{\mathbb{C}}$, alors $\left.f\right|_{K}$ est $\mu_{K}$-ergodique et, pour $\mu_{K}$-presque tout point $z \in K, \overline{\operatorname{Orb}(z)}=$ $\partial K$.

Un tel compact $K$ est appelé "hérisson"; en faisant varier la taille de $U$, on récupère une famille à 1 paramètre réel de hérissons emboités les uns dans les autres. Ces hérissons caractérisent la dynamique au sens suivant:

Proposition 4.12. Un élément $g \in \operatorname{Diff}(\mathbb{C}, 0)$ défini sur $U$ commute à $f$ si, et seulement si, $g(K)=g^{\circ(-1)}(K)=K$.

Démonstration. Si $g$ commute à $f$, il preserve les orbites de $f$ et leurs adhérence, et par suite les compacts invariants. Réciproquement, si $g$ préserve $K$, alors le groupe $G$ engendré par $f$ et $g$ aussi ; si $G$ est non abélien, la description que nous allons faire des dynamiques non abéliennes montre que $G$ ne peut pas laisser un tel compact invariant : donc $G$ est abélien. 
Par suite, le Théorème 4.11 s'applique aussi aux groupes $G$ formellement mais non analytiquement linéarisables. Un tel germe ne peut évidemment pas posséder d'intégrale première méromorphe sur $U^{*}$ à cause de l'ergodicité.

Théorème 4.13 (Naishul). Si un élément $g(z)=b z+\cdots \in \operatorname{Diff}(\mathbb{C}, 0)$ est topologiquement conjugué à un germe de difféomorphisme $f(z)=a z+\cdots$ non linéarisable comme au-dessus, alors leur nombre de rotation est le même : $a=b$.

Bien sûr, dans l'énoncé précédent, $g$ est lui aussi non linéarisable à cause de sa dynamique de hérisson.

\subsubsection{Type (Eucl) : dynamiques abéliennes résonantes}

Ici, $G$ désigne un sous-groupe non périodique du groupe de Lie abélien

$$
\mathbb{E}_{p, \lambda}=\left\{f(z)=e^{2 i \pi \frac{k}{p}} \exp \left(t X_{p, \lambda}\right) ; k \in \mathbb{Z} / p \mathbb{Z}, t \in \mathbb{C}\right\}
$$

( $G$ contient au moins un élément $f$ avec $t \neq 0$ ). La forme différentielle $\omega_{p, \lambda}=\frac{d z}{z^{p+1}}+\frac{\lambda}{2 i \pi} \frac{d z}{z}$ est invariante ; en l'intégrant, on obtient la coordonnée multiforme $w=\psi_{p, \lambda}(z)=-\frac{1}{p z^{p}}+\frac{\lambda}{2 i \pi} \log (z)$ qui redresse

- $\omega_{p, \lambda} \operatorname{sur} d w$,

- $X_{p, \lambda}$ sur $\partial_{w}$ et

- la dynamique de $\mathbb{E}_{p, \lambda}$ sur les translations.

Plus précisément, la coordonnée $w$ est une incarnation géométrique $d u$ morphisme

$$
\rho: \mathbb{E}_{p, \lambda} \rightarrow \mathbb{C} ; e^{2 i \pi \frac{k}{p}} \exp \left(t X_{p, \lambda}\right) \mapsto t .
$$

En effet, la dynamique de $\exp \left(t X_{p, \lambda}\right)$ est la translation $w \mapsto w+t$ sur les déterminations de $w$; la rotation $z \mapsto e^{2 i \pi / p} z$ échange quant à elle les déterminations de $w$. Ce type de dynamiques euclidiennes est caractéristique de l'holonomie des feuilletages définis par une 1-forme méromorphe fermée au voisinage d'un pôle multiple. En effet, on a la :

Proposition 4.14. Si un sous-groupe $G \subset \operatorname{Diff}(\mathbb{C}, 0)$ laisse invariant un germe de 1-forme méromorphe ayant un pôle multiple en 0 , alors $G$ est analytiquement équivalent à un sous-groupe de $\mathbb{E}_{p, \lambda}$ pour des $p \in \mathbb{N}^{*}$ et $\lambda \in \mathbb{C}$ convenables.

Démonstration. On redresse la 1-forme à l'aide de la Proposition 2.3 sur un modèle $\omega_{p, \lambda}$ puis on remarque que si $f$ commute à $\omega_{p, \lambda}$, alors $f$ commute à $X_{p, \lambda}$ et à son flot; on conclut avec la Proposition 2.17.

Remarque 4.15. On déduit facilement du Lemme 4.6 les différents types d'adhérence possibles $\bar{G}$ pour un sous-groupe $G \subset \mathbb{E}_{p, \lambda}$. L'adhérence des orbites de $G$ contient les orbites de $\bar{G}$ par le même type d'argument que celui de la remarque 4.8. On a les possibilités suivantes 
- $\overline{\operatorname{Orb}\left(z_{0}\right)}=\operatorname{Orb}\left(z_{0}\right)$ est discrète dans $U^{*}$ et accumule 0 ,

- $\overline{\mathrm{Orb}\left(z_{0}\right)}$ est analytique de dimension 1 dans $U^{*}$,

$-\overline{\operatorname{Orb}\left(z_{0}\right)}=U$.

Notons que le type d'orbite est indépendant du choix de $z_{0}$ ou des générateurs : il ne dépend que de $G$. On se ramène encore au Lemme 4.6 en se plaçant dans la variable $w$ ci-dessus en tenant compte de $\rho(G)$ et, lorsque celui-ci contient un réseau, de la monodromie $w \mapsto w+\lambda$ de la coordonnée $w=-\frac{1}{p z^{p}}+\frac{\lambda}{2 i \pi} \log (z)$ (voir Remarque 4.16).

Remarque 4.16. Si un sous-groupe $G \subset \mathbb{E}_{p, \lambda}$ contient "deux translations" $\mathbb{R}$-indépendantes, $f=\exp \left(t X_{p, \lambda}\right)$ et $g=\exp \left(s X_{p, \lambda}\right)$ avec $t / s \notin \mathbb{R}$, alors la description faite dans l'exemple 3.17 nous montre que la dynamique de $h=e^{2 i \pi / p} \exp \left(\frac{\lambda}{p} X_{p, \lambda}\right)$ resurgit à travers les grands commutateurs $\left[f^{\circ( \pm N)}, g^{\circ( \pm N)}\right], N>>0$; par suite, en itérant $p$ fois un tel commutateur, on retrouve aussi $h^{\circ p}=\exp \left(\lambda X_{p, \lambda}\right)$. On ne modifie donc pas les orbites en rajoutant $h$ aux générateurs de $G$. Par conséquent, si $t$, s et $\lambda$ sont suffisamment généraux, alors la dynamique induite par $G$ est à orbites denses (excepté le point fixe 0). Par contre, lorsque $t, s$ et $\lambda$ engendrent un $\mathbb{Z}$ module de rang 2 , alors les orbites sont discrete et le quotient d'un voisinage épointé $U^{*}$ par la dynamique est un tore; dans ce cas, on construit, à l'aide de la fonction de Weierstrass associée, une fonction invariante méromorphe sur $U^{*}$.

Remarque 4.17. On peut conjuguer $f=\exp \left(X_{p, \lambda}\right) \grave{a} g=\exp \left(X_{p, \mu}\right)$ par un homéomorphisme qui est analytique en dehors de 0 dès que $\lambda$ et $\mu$ sont dans le demi-plan supérieur $\mathbb{H}$. En effet, il suffit de choisir convenablement une conjugaison $\mathbb{R}$-linéaire de la forme $w \mapsto w_{1}+\alpha w_{2}$ sur les cartes de l'atlas où $w=w_{1}+i w_{2}$ et $\alpha \in \mathbb{C}$, Ré $(\alpha)>0$. Par contre, si $\lambda$ est réel et si $f$ est conjuguée à $g$ par un homéomorphisme analytique en dehors de 0 , alors $\mu=\lambda$. En effet, une telle conjugaison induit un homéomorphisme entre les chapelets; choisissons les recollements $\varphi_{k}^{0}$ et $\varphi_{k}^{\infty}$ tous triviaux sauf disons $\varphi_{0}^{0}(\tau)=e^{2 i \pi \lambda} \tau$. Alors les homéomorphismes sur les sphères sont analytiques en dehors de 0 et $\infty$ et doivent coïncider sur les $2 p-1$ recollements triviaux et donc partout. Ils induisent alors une conjugaison topologique entre $e^{2 i \pi \lambda} \tau$ et $e^{2 i \pi \mu} \tau$; comme ces germes sont des rotations, il vient $\mu=\lambda$.

\subsubsection{Type (Eucl) ${ }^{\text {ex }}$ : dynamiques euclidiennes excep- tionnelles}

On suppose ici que $G$ est formellement conjugué à un sous-groupe de $\mathbb{E}_{p, \lambda}$ pour des $p \in \mathbb{N}^{*}$ et $\lambda \in \mathbb{C}^{*}$ mais la transformation normalisante $\hat{\varphi}$ diverge. Rappelons alors (voir Proposition 3.39) que $\hat{\varphi}_{*} G$ est alors engendré par

$$
\hat{\varphi}_{*} f=\exp \left(X_{p, \lambda}\right) \quad \text { et } \quad \hat{\varphi}_{*} g=e^{\frac{2 i \pi}{q}} \cdot \exp \left(\frac{n}{q} X_{p, \lambda}\right)
$$


où $\lambda \in \mathbb{C}, p=k q$ avec $k, q \in \mathbb{N}^{*}$ et $n \in \mathbb{Z}$.

Proposition 4.18. Il existe un homéomorphisme $\Psi:(\mathbb{C}, 0) \rightarrow(\mathbb{C}, 0)$ qui conjugue les générateurs de $G$ ci-dessus respectivement à

$$
f_{0}=\exp \left(X_{p, 0}\right) \quad \text { et } \quad g_{0}=e^{\frac{2 i \pi}{q}} \exp \left(\frac{n}{q} X_{p, 0}\right) .
$$

Démonstration. On procède comme dans la preuve du Théorème 3.34 en remplaçant le chapelet de $p$ sphères associé à $f$ par le chapelet de $k$ sphère obtenu en le quotientant par l'action de $g$ : c'est l'espace des orbites de $G$. On le conjugue topologiquement à celui de $G_{0}=<f_{0}, g_{0}>$, puis on relève la conjugaison sur $U$.

Remarque 4.19. Le groupe $G_{0}$ agit discrètement sur le voisinage épointé $U^{*}$; il possède en fait l'intégrale première $H_{0}=e^{2 i \pi / p z^{p}}$ qui est holomorphe en dehors de 0 . Le groupe $G$ agit lui aussi discrètement sur $U^{*}$ mais ne possède en général pas d'intégrale première même méromorphe sur $U^{*}$. En effet, une telle intégrale première définirait des fonctions méromorphes $F_{k}^{ \pm}$ sur les sphères du chapelet associé satisfaisant les conditions de recollement comme par exemple $F_{k}^{-} \circ \varphi_{k}^{0}=F_{k}^{+}$; si l'on choisit $\varphi_{k}^{0}$ ayant un continuum de singularités sur un cercle $|\tau|=r$, alors on aboutit à une contradiction. Cependant, on trouve dans [220] l'exemple suivant. Considérons le germe $f$ formellement conjugué à $\exp \left(X_{1,0}\right)$ dont les invariants sont $\varphi^{0}(\tau)=\tau$ et $\varphi^{\infty}(\tau)=\tau+1$; alors les fonctions holomorphes définies sur les sphères $d u$ chapelet par $F^{ \pm}(\tau)=e^{2 i \pi \tau}$ sont compatibles avec les applications de recollement et définissent une intégrale première holomorphe sur $U^{*}$ (dont la croissance est surexponentielle à l'origine). Ici, le chapelet est affine en ce sens que $\varphi^{0}$ et $\varphi^{\infty}$ le sont dans la variable $\tau$; cependant, on peut construire des exemples plus exotiques comme le suivant qui m'a été communiqué par Jean Écalle. On considère encore un germe $f$ formellement conjugué à $\exp \left(X_{1,0}\right)$ et on commence par fixer des polynômes $F^{ \pm}$sur les sphères tous deux de la forme $a_{n} z^{n}+a_{n+1} z^{n+1}+\cdots+a_{N} z^{N}$ avec $0<n<N$ et $a_{n}, a_{N} \neq 0$; alors on peut trouver des germes de difféomorphismes $\varphi^{0}$ en 0 et $\varphi^{\infty}$ à l'infini conjugant les germes de fonctions induits par $F^{+}$et $F^{-}$. Les difféomorphismes $f$ ainsi construits admettent une intégrale première holomorphe sur $U^{*}$, à croissance exponentielle d'ordre fini $N$ en 0.

\subsubsection{Type (Aff) : dynamiques affines}

On considère ici des sous-groupes $G$ du groupe de Lie :

$$
\mathbb{A}_{p}=\left\{f(z)=a z /\left(1-b z^{p}\right)^{1 / p} ; a \in \mathbb{C}^{*}, b \in \mathbb{C}\right\}
$$

avec $p \in \mathbb{N}^{*}$, où l'on suppose de plus que $G$ n'est pas abélien pour ne pas retomber dans une des situations précédentes. Chaque élément $f \in \mathbb{A}_{p}$ 
envoit la 1-forme $\omega_{p}=\omega_{p, 0}=\frac{d z}{z^{p+1}}$ sur un multiple constant, $f_{*} \omega_{p}=a^{p} \cdot \omega_{p}$. En intégrant cette 1-forme, on obtient une coordonnée $w=-\frac{1}{p z^{p}}$ dans laquelle la dynamique devient affine $: f(w)=\tilde{a} w+\tilde{b}=a^{-p} w+\frac{b}{p a^{p}}$. Cette coordonnée est l'incarnation géométrique du morphisme

$$
\rho: \mathbb{A}_{p} \rightarrow \operatorname{Aff}(\mathbb{C}) ; a z /\left(1-b z^{p}\right)^{1 / p}=a z+a \frac{b}{p} z^{p+1}+\cdots \mapsto a^{-p} w+\frac{b}{p a^{p}}
$$

considéré dans la preuve du Théorème 2.22.

Proposition 4.20. Si un sous-groupe $G \subset \operatorname{Diff}(\mathbb{C}, 0)$ préserve le $\mathbb{C}$-espace vectoriel engendré par un germe de 1-forme méromorphe $\omega$ non triviale, i.e. $f_{*} \omega=c_{f} \cdot \omega, c_{f} \in \mathbb{C}$ pour tout $f \in G$, sans pour autant préserver $\omega$, alors $G$ est analytiquement redressable sur un sous-groupe non abélien de $\mathbb{A}_{p}$ pour un $p \in \mathbb{N}^{*}$.

Démonstration. L'hypothèse qu'il existe au moins un élément $f \in G$ tel que $f_{*} \omega \neq \omega$ entraine immédiatement, par un calcul à l'ordre 1 , que $\omega$ doit avoir un pôle multiple en 0 . On redresse alors $\omega$ sur $\omega_{p, \lambda}$ par la Proposition 2.3 et on conclut par le même calcul que dans la fin de la preuve du Théorème 2.22 .

Proposition 4.21. Un sous-groupe non abélien $G \subset \mathbb{A}_{p}$ est à orbites discrètes sur un voisinage épointé $U^{*}$ de 0 si, et seulement si, à conjuguaison près, $G$ est l'un des groupes suivants

- $G=<a z, z /\left(1-z^{p}\right)^{1 / p}, z /\left(1-\tau z^{p}\right)^{1 / p}>$, où $a^{p}=-1$ et $\tau \in \mathbb{C} \backslash \mathbb{R}$; une intégrale première est donnée par $\wp_{\tau}\left(\frac{1}{z^{p}}\right)$.

- $G=<a z, z /\left(1-z^{p}\right)^{1 / p}>$, où $a^{p}$ est d'ordre $2,3,4$ ou 6 ; une intégrale première est respectivement donnée par : $\cos \left(\frac{2 \pi}{z^{p}}\right), \wp_{j}^{\prime}\left(\frac{1}{z^{p}}\right)$, $\left(\wp_{i}\left(\frac{1}{z^{p}}\right)\right)^{2}$ et $\left(\wp_{j}^{\prime}\left(\frac{1}{z^{p}}\right)\right)^{2}$.

Ces intégrales premières sont méromorphes sur $U^{*}$ et séparent les orbites.

Démonstration. L'application $z \mapsto w=-\frac{1}{p z^{p}}$ étant un revêtement fini, l'action de $G$ est dicrète si, et seulement si, l'action de $\rho(G)$ l'est. Les sousgroupes discrets non abéliens de $\operatorname{Aff}(\mathbb{C})$ sont les groupes de pavages dont on tire la liste de l'énoncé.

Remarque 4.22. Encore en utilisant la coordonnée affine, on déduit que pour un sous-groupe $G \subset \mathbb{A}_{p}$ non discret, l'adhérence des orbites est lisse et analytique réelle sur $U^{*}$.

\subsubsection{Type (Aff) ${ }^{\text {ex }}$ : dynamiques affines exceptionnelles}

On suppose ici que $G$ est formellement conjugué à un sous-groupe de $\mathbb{A}_{p}$ pour un $p \in \mathbb{N}^{*}$ mais la transformation normalisante $\hat{\varphi}$ diverge. Rappelons alors (voir Proposition 3.39) que $\hat{\varphi}_{*} G$ est engendré par

$$
f_{0}(z)=z /\left(1-z^{p}\right)^{1 / p} \quad \text { et } \quad g_{0}(z)=e^{\frac{2 i \pi}{2 q}} z
$$


où $p, q \in \mathbb{N}^{*}$ avec $p=k q, k$ impair.

Proposition 4.23. Il existe un homéomorphisme $\Psi:(\mathbb{C}, 0) \rightarrow(\mathbb{C}, 0)$ qui conjugue $G$ à son modèle formel $G_{0}$, engendré par $f_{0}$ et $g_{0}$.

Démonstration. On procède comme dans la preuve de la Proposition 4.18.

Remarque 4.24. Le groupe $G_{0}$ possède l'intégrale première $H_{0}(z)=$ $\cos \left(\frac{2 \pi}{p z^{p}}\right)$ qui est holomorphe sur $U^{*}$.

\subsection{Dynamiques non résolubles}

Dans cette section, $G$ est un sous-groupe non résoluble de type fini de $\operatorname{Diff}(\mathbb{C}, 0)$. Les premiers résultats que l'on trouve dans la littérature sur la dynamique induite par un groupe $G$ "générique" sur un voisinage de 0 supposent que la partie linéaire

$$
\left\{a \in \mathbb{C}^{*} ; \exists f \in \hat{G}, f(z)=a z+\cdots\right\}
$$

est un sous-groupe dense de $\mathbb{C}^{*}$. Yulij Ilyashenko montre notamment dans [91] (voir aussi [50]) que, sous cette hypothèse :

- toute orbite (autre que 0 ) est dense dans le voisinage $U$ de 0 ,

- tout homéomorphisme conjugant $G$ à un autre sous-groupe $\operatorname{de~} \operatorname{Diff}(\mathbb{C}, 0)$ est conforme ou anti-conforme,

- l'ensemble des points $z_{0} \in U^{*}$ fixés par un élément non trivial de $G$ est dense dans $U$.

Un premier problème soulevé dans [91] est que l'hypothèse sur la partie linéaire n'est pas ouverte, elle n'est pas stable par perturbation. Un second problème est que les singularités de feuilletages les plus simples dont l'holonomie est non résoluble sont les singularités définies par des champs de vecteurs sur $\left(\mathbb{C}^{2}, 0\right)$ avec partie linéaire nilpotente $X=y \partial_{x}+\cdots$. Or les générateurs des groupes d'holonomie correspondant ont une partie linéaire périodique (d'ordre fini). Les démonstrations de [91] utilisent fortement l'existence de contractions et deviennent inopérantes pour décrire la dynamique transverse des champs $X$ nilpotents comme au-dessus.

D'après le Théorème 2.22 , un sous-groupe non résoluble $G \subset \operatorname{Diff}(\mathbb{C}, 0)$ contient toujours deux éléments $f, g \in G$ de la forme :

$$
f(z)=z+c z^{p+1}+\cdots \quad \text { et } \quad g(z)=z+d z^{q+1}+\cdots
$$

où $c, d \in \mathbb{C}^{*}$ et $0<p<q$. C'est à partir du sous-groupe engendré par ces deux éléments que A.A. Shcherbakov puis I. Nakai généralisent les résultats d'Ilyashenko à tous les sous-groupes non résolubles. Précisément : 
Théorème 4.25 (Shcherbakov). Soit $G$ le pseudo-groupe induit sur un petit voisinage ouvert $U$ de $0 \in \mathbb{C}$ par un sous-groupe non résoluble de type fini de $\operatorname{Diff}(\mathbb{C}, 0)$, une famille de générateurs étant choisie. Alors, quitte à diminuer $U$ :

1. il existe une collection finie d'ouverts disjoints dont l'adhérence recouvre $U$ telle que $G$ agit densément sur chacun de ces ouverts;

2. si $\varphi: U \hookrightarrow \mathbb{C}$ est un homéomorphisme de $U$ sur son image, disons préservant l'orientation et fixant 0 , envoyant la dynamique de $G$ sur celle d'un pseudo-groupe de transformations encore conformes, alors $\varphi$ est conforme;

3. l'ensemble des points fixés par un élément non trivial de $G$ :

$$
\left\{z_{0} \in U ; \exists g \in G, g \neq i \text { dentité et } g\left(z_{0}\right)=z_{0}\right\}
$$

est dense dans $U$.

Les assertions (1), (2) et (3) apparaissent respectivement dans [203], [205] et [206]. La preuve de (3) n'apparaît qu'en 1998 dans [207].

L'approche nouvelle apportée par Isao Nakai dans [151] permet, outre la simplification des démonstrations des propriétés précédentes, de préciser les domaines de densité des orbites :

Théorème 4.26 (Nakai). Soit $G$ comme précédemment. Alors on est dans l'une des deux situations suivantes:

- il existe une coordonnée z et un entier $p \in \mathbb{N}^{*}$ tels que l'ensemble $\Sigma=\left\{z^{p} \in \mathbb{R}\right\}$ est invariant par $G$, et $G$ agit densément sur chacune des composantes connexes de $\Sigma \backslash\{0\}$ et de $U \backslash \Sigma$ (voir Figure 4.1); - $G$ agit densément sur $U^{*}:=U \backslash\{0\}$.

Dans le premier cas, $G$ est (réel et) le relevé par $z \mapsto z^{p}$ d'un sous-groupe de $\operatorname{Diff}(\mathbb{R}, 0)$.

Non seulement cet énoncé nous décrit de façon on ne peut plus précise les ouverts de densité de (1), mais il nous montre en plus que la situation générique (dense et ouverte pour la topologie de Krull) est la densité sur $U^{*}$ (voir Remarque 2.29). Les secteurs de $U \backslash \Sigma$ et les demi-droites de $\Sigma \backslash\{0\}$ sont appelés secteurs et séparatrices de Nakai.

Toutes ces démonstrations reposent sur l'existence de flots conformes dans l'adhérence $\bar{G}$ de $G$ pour la topologie de convergence uniforme. Là où ces flots sont transitifs, les orbites de $G$ sont denses.

Notons que les propriétés obtenues sont aussi celles de la dynamique d'un sous-groupe générique de $\mathbb{A}_{p}$. En étudiant plus précisément l'algèbre de Lie associée à $\bar{G}$, on démontre le résultat suivant (on pose $\Sigma=\{0\}$ lorsqu'il n'y a pas de séparatrice) :

Théorème 4.27 (Belliart,Liousse,Loray). Soit $G$ comme précédemment. Alors tout germe $f:\left(\mathbb{C}, z_{0}\right) \rightarrow\left(\mathbb{C}, z_{1}\right)$ d'application conforme entre deux 


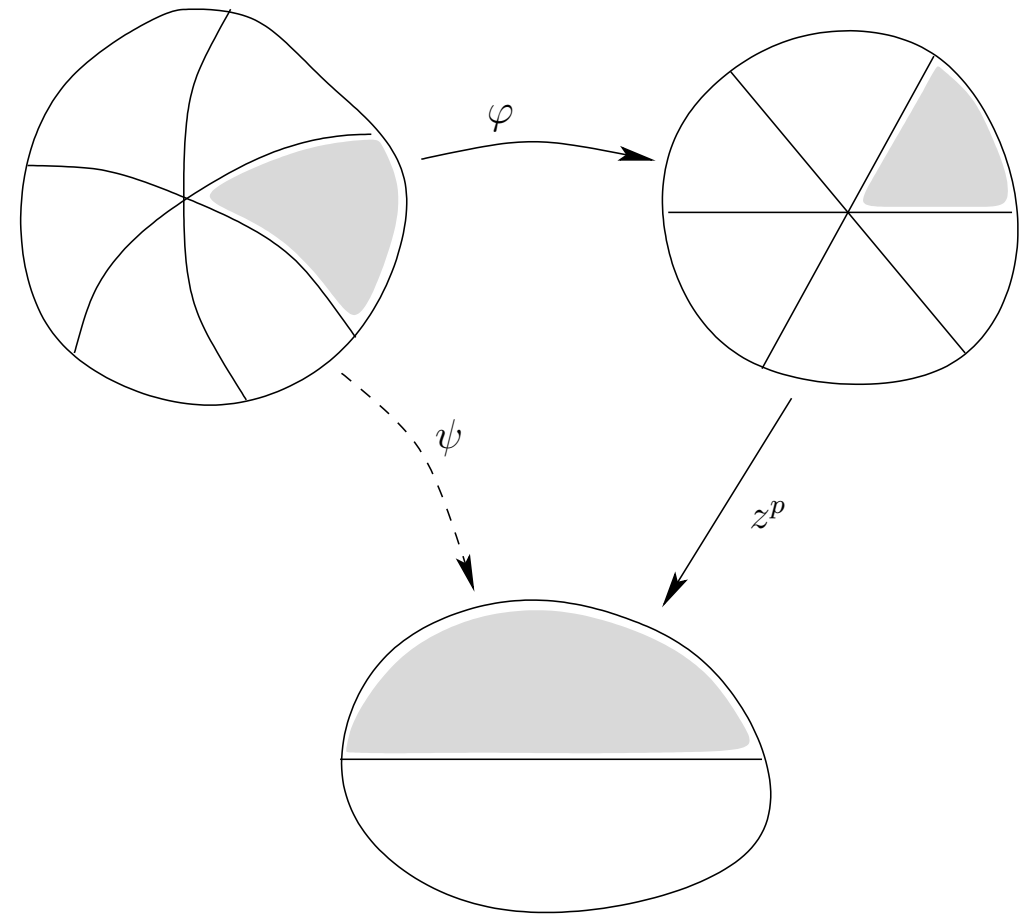

FIgURE 4.1 - Les secteurs de Nakai 
points $z_{0}$ et $z_{1}$ appartenant à une même composante connexe de $U \backslash \Sigma$ (resp. de $\Sigma \backslash\{0\}$ préservant $\Sigma$ ) est approché uniformément sur un voisinage $V$ de $z_{0}$ par une suite d'éléments $\varphi_{n}: V \rightarrow U$ du pseudo-groupe induit par $G$ sur $U$.

En d'autres termes, la clôture $\bar{G}$ de $G$ pour la convergence uniforme est, en restriction à chaque secteur, le pseudo-groupe (de dimension infinie) de toutes les transformations conformes. En particulier, cette description tranche définitivement avec la description des dynamiques résolubles. Une conséquence immédiate est qu'aucune structure géométrique autre que la structure conforme n'est préservée par ce type de dynamique. Par structure géométrique, on entend ici une forme différentielle, une métrique, un tenseur, etc... Bref, tout objet issu de la géométrie différentielle. Une telle dynamique transverse signifiera pour le feuilletage une forte non intégrabilité.

\subsection{Le Théorème de de Nakai}

Soient $f(z)=z+c z^{p+1}+\cdots$ et $g(z)=z+d z^{q+1}+\cdots$ deux éléments de $\operatorname{Diff}(\mathbb{C}, 0)$ avec $c, d \in \mathbb{C}^{*}, p, q \in \mathbb{N}^{*}$ et $p<q$. Considérons un pétale attractif $V$ pour $f$ et une coordonnée de Leau $\psi: V \hookrightarrow \mathbb{C}$ associée. Considérons la dynamique induite par $g$ dans cette coordonnée (voir Figure 4.2).

Tout d'abord, puisque $g$ est plus tangente à l'identité que $f$, sa dynamique est plus lente : au fûr et à mesure que l'on s'approche de l'infini, la dynamique de $\psi_{*} g$ ralentit puisque $\psi$ est la coordonnée dans laquelle l'ordre de grandeur du déplacement de $f$ est constant. Aussi, on vérifie facilement que :

$$
\lim _{n \rightarrow+\infty} \psi_{*} g(w+n)-n=\text { identité, }
$$

ce qui signifie, lorsqu'on retourne dans la variable $z$ de départ, que :

$$
\lim _{n \rightarrow+\infty} f^{\circ(-n)} \circ g \circ f^{\circ n}=\text { identité, }
$$

la convergence étant uniforme sur le pétale $V$.

Une autre remarque suggérée par la figure 4.2 est que les "courbes" le long desquelles se déplace la dynamique de $\psi_{*} g$ ressemblent à des droites parallèles lorsque l'on s'approche de l'infini dans la direction horizontale. L'idée de Nakai est de renormaliser la dynamique de $g$, dans le processus itératif précédent, en l'itérant d'autant plus que l'on s'approche de l'infini par $f$, de façon à récupérer par passage à la limite une dynamique de translation le long de ces droites parallèles :

$$
\lim _{n \rightarrow+\infty} \psi_{*} g^{\circ k_{n}}(w+n)-n=w+\alpha
$$




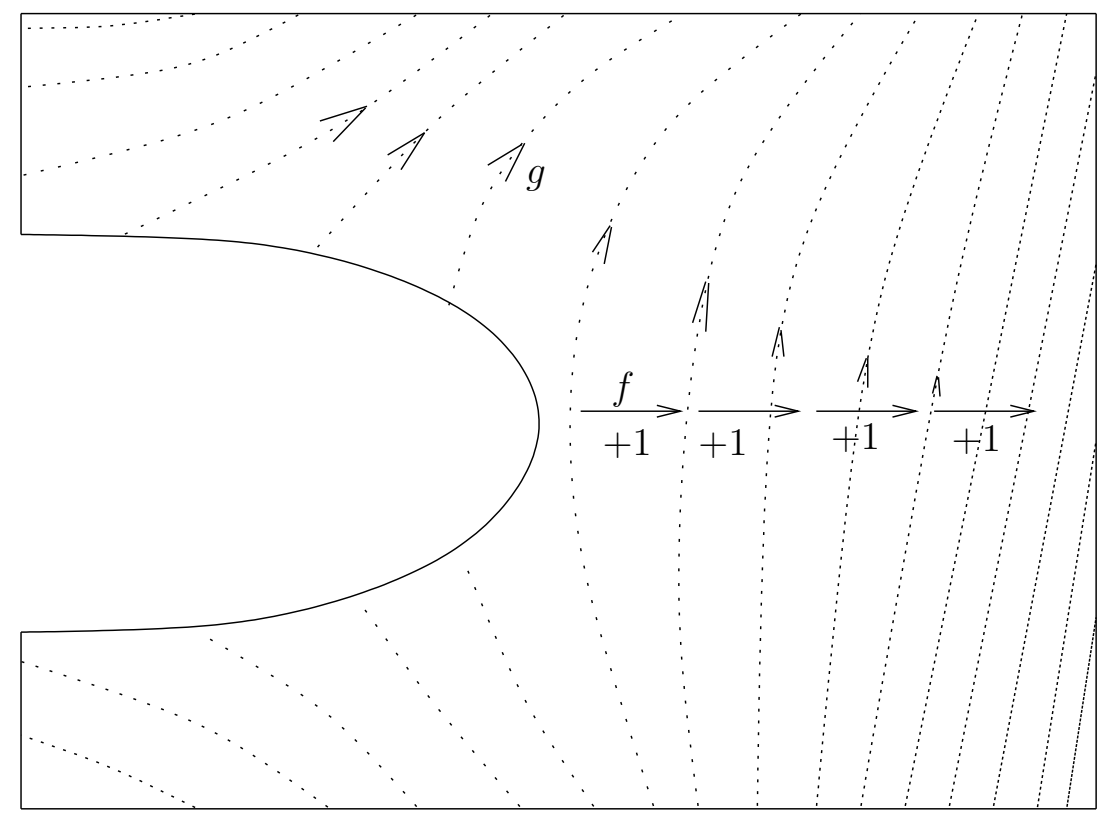

Figure 4.2 - La dynamique de $g$ dans les coordonnées trivialisantes de $f$ 
pour une suite $k_{n}$ d'entiers positifs adéquate et une constante de translation $\alpha \in \mathbb{C}^{*}$. Dans la variable $z$, ceci signifie que :

$$
\lim _{n \rightarrow+\infty} f^{\circ(-n)} \circ g^{\circ k_{n}} \circ f^{\circ n}=f^{\circ \alpha}
$$

où $f^{\circ \alpha}$ désigne l'itérée complexe d'ordre $\alpha$ de $f$ sur $V$. Maintenant, la suite $k_{n}$ est certainement croissante et on peut espérer récupérer l'itérée complexe d'ordre $t \cdot \lambda, t \in \mathbb{R}$, par convergence de :

$$
\lim _{n \rightarrow+\infty} f^{\circ(-n)} \circ g^{\circ\left[t k_{n}\right]} \circ f^{\circ n}=f^{\circ t \alpha}
$$

où $\left[t k_{n}\right]$ désigne la partie entière de $t k_{n}$. Bien sûr, dans tout ce que l'on vient de dire, il faut certainement se restreindre à un sous-pétale $V_{t}$ de $V$ pour que les approximantes $f^{\circ(-n)} \circ g^{\circ\left[t k_{n}\right]} \circ f^{\circ n}$ et la limite $f^{\circ t \alpha}$ soient bien définies : $V_{t} \hookrightarrow V$.

Lemme 4.28 (Nakai). Soient $f(z)=z+c z^{p+1}+\cdots$ et $g(z)=z+$ $d z^{q+1}+\cdots$ deux éléments de $\operatorname{Diff}(\mathbb{C}, 0)$ avec $c, d \in \mathbb{C}^{*}, 0<p<q$ et $V$ un pétale attractif de $f$. Notons $X$ le générateur infinitésimal de $f$ sur $V, \alpha:=\frac{-p d}{(-p c)^{q / p}}$ et $k_{n}:=n^{\frac{q}{p}-1}$ pour $n \in \mathbb{N}^{*}$. Alors pour tout $T>0$, il existe un sous-pétale $V_{T} \subset V$ sur lequel, pour tout $\left.t \in\right]-T, T$, le flot au temps $t$ du champ de vecteurs holomorphe $\alpha X$ est bien défini sur $V_{T}$, $\exp (t \alpha X): V_{T} \rightarrow V$, et approché uniformément sur tout compact de $V_{T}$ par la suite de transformations (bien définies pour $n$ suffisamment grand) :

$$
f^{\circ(-n)} \circ g^{\circ\left[t k_{n}\right]} \circ f^{\circ n}: V_{T} \rightarrow V
$$

(où $\left[t k_{n}\right]$ est la partie entière de $t k_{n}$ ).

De plus, pour $T>0$ fixé, la collection des sous-pétales $V_{T}$ lorsque $V$ décrit les pétales de $f$ est encore un recouvrement d'un voisinage épointé de l'origine.

Démonstration grossière. On a d'abord l'estimation grossière suivante des itérées $f^{\circ n}(z)$ lorsque $z$ appartient à un compact $K \subset V$ du pétale :

$$
f^{\circ n}(z)=z+n c z^{p+1}+\cdots \sim \frac{z}{\left(1-n p c z^{p}\right)^{1 / p}} \sim \frac{1}{(-n p c)^{1 / p}}
$$

le choix de la racine $p^{\text {ème }}$ dépendant de la direction du pétale. Toutes les orbites arrivent avec une direction asymptotique au voisinage de laquelle $f$ et $g$ sont les flots au temps 1 de champs de vecteurs holomorphes $X \sim$ $c z^{p+1} \partial_{z}$ et $Y \sim d z^{q+1} \partial_{z}$. Si $\omega$ désigne la 1 -forme duale de $X$, alors il vient :

$$
\omega(Y) \sim \frac{d}{c} z^{q-p}
$$


ce qui signifie que $g(z)$ est comparable à $f^{\circ \frac{d}{c} z^{q-p}}(z)$. En appliquant cette dernière estimation à $f^{\circ n}(z)$, il vient

$$
\omega\left(\left(f^{\circ n}\right)^{*} Y\right)=\left(f^{\circ n}\right)^{*} \omega(Y) \sim \frac{d}{c}(-n p c)^{1-\frac{q}{p}} .
$$

Ainsi, $\left(f^{\circ n}\right)^{*} n^{\frac{q}{p}-1} Y$ est de l'ordre de $\frac{d}{c}(-p c)^{1-\frac{q}{p}} X$ et le résultat se déduit en passant aux exponentielles.

La preuve qui suit consiste essentiellement à justifier, en travaillant dans la coordonnée de Leau $\psi$ associée à $f$ sur $V$, les approximations faites le long de la démonstration précédente. Afin d'estimer $\psi_{*} g$, il nous faut d'abord estimer $\psi$ et $\psi^{\circ(-1)}$. Pour cela, remarquons qu'à une constante additive près, $\psi$ admet la décomposition :

$$
\psi=\psi_{p, \lambda} \circ \varphi=\Lambda_{\frac{\lambda}{2 i \pi p}} \circ \psi_{p, 0} \circ \varphi
$$

où $\varphi$ est la normalisante sectorielle qui conjugue $f$ à son modèle formel $\exp \left(X_{p, \lambda}\right)$ et :

$$
\Lambda_{\lambda}(w):=w-\lambda \log (w) .
$$

Puisque $\varphi$ et $\varphi^{\circ(-1)}$ admettent tous deux des développements asymptotiques, il nous manque essentiellement l'estimation du :

Lemme 4.29. L'inverse de la transformation $\Lambda_{\lambda}(w)=w-\lambda \log (w)$, près de l'infini, est du type :

$$
\Lambda_{\lambda}^{\circ(-1)}(w)=\Lambda_{-\lambda}(w)+O\left(\frac{\log (|w|)}{|w|}\right) .
$$

Démonstration. Remarquons tout d'abord que :

$$
\frac{|w-\lambda \log (w)|}{|w|} \rightarrow 1 \quad \text { quand } \quad|w| \rightarrow+\infty .
$$

Ainsi, il existe une constante $C>0$ telle que :

$$
\left|w-\Lambda_{\lambda}(w)\right|<C \log \left(\left|\Lambda_{\lambda}(w)\right|\right),
$$

ce qui, en composant par $\Lambda_{\lambda}^{\circ(-1)}$, nous donne une première estimation :

$$
\left|\Lambda_{\lambda}^{\circ(-1)}(w)-w\right|<C \log (|w|) .
$$

Maintenant, en développant $\Lambda_{-\lambda} \circ \Lambda_{\lambda}$, il vient :

$$
\Lambda_{-\lambda} \circ \Lambda_{\lambda}=w+\lambda \log \left(1-\lambda \frac{\log (w)}{w}\right)=z+O\left(\frac{\log (|w|)}{|w|}\right)
$$


ce qui, en composant par $\Lambda_{\lambda}^{\circ(-1)}$, nous donne :

$$
\Lambda_{-\lambda}=\Lambda_{\lambda}^{\circ(-1)}+O\left(\frac{\log \left(\left|\Lambda_{\lambda}^{\circ(-1)}\right|\right)}{\left|\Lambda_{\lambda}^{\circ(-1)}\right|}\right) .
$$

La première estimation de $\Lambda_{\lambda}^{\circ(-1)}$ nous donne le résultat.

Maintenant, un calcul direct nous donne le :

Lemme 4.30. Avec les notations précédentes, on a:

$$
\psi_{*} g(w)=w+\alpha w^{1-\frac{q}{p}}+o\left(|w|^{1-\frac{q}{p}}\right)
$$

et dans la variable $\tilde{w}:=\alpha^{-1} w^{q / p}$, la dynamique de $g$ est du type :

$$
\tilde{g}(\tilde{w})=\tilde{w}+1+o\left(|\tilde{w}|^{0}\right) .
$$

Ici et dans toute la suite, $o\left(|z|^{\delta}\right)$ désigne, pour $\delta \in \mathbb{R}$, une fonction arbitraire $R(z)$ satisfaisant $|R(z)| \leq C|z|^{\delta-\varepsilon}$ pour des constantes $C, \varepsilon>0$. Dans la variable $\tilde{w}$, on peut estimer facilement l'itérée $N^{\text {ème }}$ de $g$ :

Lemme 4.31. Si $\tilde{g}(\tilde{w})=\tilde{w}+1+O\left(\frac{1}{|\tilde{w}|^{k / q}}\right)$, pour un $k>0$, alors sur un pétale attractif pour $\tilde{g}$, on $a$ :

$$
\left|\tilde{g}^{\circ N}(\tilde{w})-\tilde{w}-N\right| \leq\left\{\begin{array}{cl}
C\left[(|\tilde{w}|+N)^{1-\frac{k}{q}}-|\tilde{w}|^{1-\frac{k}{q}}\right] & \text { pour } k<q \\
C\left[\log \left(1+\frac{N}{|\tilde{w}|}\right)\right] & \text { pour } k=q \\
C\left[|\tilde{w}|^{1-\frac{k}{q}}-(|\tilde{w}|+N)^{1-\frac{k}{q}}\right] & \text { pour } k>q
\end{array}\right.
$$

pour une constante $C>0$. Si, de plus, on ne s'interesse qu'à des valeurs de $N$ telles que $\frac{N}{|\tilde{w}|}$ est uniformément borné, alors les estimations précédentes restent vraies au voisinage de l'infini et se réécrivent:

$$
\tilde{g}^{\circ N}(\tilde{w})=\tilde{w}+N+O\left(\frac{N}{|\tilde{w}|^{k / q}}\right) .
$$

Démonstration. Une première estimation grossière démontrée dans le Théorème de la Fleur 3.13 est :

$$
\left|\tilde{g}^{\circ N}(\tilde{w})\right| \geq C|\tilde{w}+N|
$$

pour une constante $C>0$, sur un pétale attractif pour $g$. Maintenant, puisque :

$$
|\tilde{g}(\tilde{w})-\tilde{w}-1| \leq C|\tilde{w}|^{-k / q}
$$


on a :

$$
\left|\tilde{g}^{\circ N}(\tilde{w})-\tilde{w}-N\right| \leq C^{\underline{t e}} \sum_{n=0}^{N-1}(|\tilde{w}|+n)^{-k / q} \leq C^{\underline{t e}} \int_{0}^{N}(|\tilde{w}|+t)^{-k / q} d t
$$

d'où les trois estimations, sur tout pétale attractif. $\mathrm{Si} \frac{N}{|\tilde{w}|}$ reste borné, on déduit dans les trois cas $(k<q, k=q$ ou $k>q)$ la dernière estimation. Elle se montre de la même manière sur un pétale répulsif de $g$ en démarrant de l'estimation grossière :

$$
\left|\tilde{g}^{\circ N}(\tilde{w})\right| \geq C|\tilde{w}-N| .
$$

Lemme 4.32. De retour dans la variable $w$, sur le sous-pétale $V_{T} \subset V$ défini par :

$$
V_{T}:=\{w \in V ; \operatorname{dist}(w, \partial V)>M\}
$$

pour un $M>0$, les transformations $\psi_{*} g^{\circ\left[t \cdot k_{n}\right]}(w+n)-n$ sont bien définies sur $V_{T}$ à valeurs dans $V$ pour tout $n>>0$ et tout $\left.t \in\right]-T, T[$ (et donc forment une famille normale) et satisfont :

$$
\psi_{*} g^{\circ\left[t \cdot k_{n}\right]}(w+n)-w-n=t \alpha\left(1+\frac{w}{n}\right)^{1-\frac{q}{p}}+O\left(\frac{1}{n^{\varepsilon}}\right)
$$

pour un $\varepsilon>0$.

Démonstration. On utilise des estimations grossières pour construire $V_{T}$. Sur le pétale $V$, on a $|w+n|>R+\vartheta n$ où $R=\operatorname{dist}(V, 0)$ et $\vartheta \in] 0,1[$ est d'autant plus petit que le pétale est ouvert. Dans la variable $\tilde{w}:=$ $\alpha^{-1} w^{q / p}$, nous allons donc appliquer la transformation $\tilde{g}^{\circ N}$ en des points $\tilde{w}$ satisfaisant $|\tilde{w}|>C \underline{\underline{t e}} n^{q / p}$ pour des $N<C \underline{\underline{t e}} n^{\frac{q}{p}-1}$ de sorte que $\frac{N}{|\tilde{w}|}$ va rester borné et que le lemme 4.31 va s'appliquer. Dans un premier temps, nous nous contentons du fait que $\tilde{g}^{\circ N}(\tilde{w})$ est contenu dans le disque de centre $\tilde{w}$ et de rayon $(1+\varepsilon) N$. Dans la variable $w$, ce disque est applati et son plus grand rayon est donné par celui qui se dirige vers $w=0$; on en déduit que $\psi_{*} g^{\circ N}(w+n)$ est contenu dans le disque de centre $w+n$ et de rayon :

$$
R+\vartheta n-|\alpha|\left[\frac{(R+\vartheta \cdot n)^{q / p}}{|\alpha|}-\frac{q}{p}(1+\varepsilon) T n^{\frac{q}{p}-1}\right]^{p / q}
$$

qui est visiblement borné. De plus, pour $n>>0$, la borne $M$ est du type $(1+\varepsilon) \vartheta^{1-\frac{q}{p}} T|\alpha|$ pour un $\varepsilon$ un peu plus grand que celui de départ. Si le pétale $V$ est, dans la variable $w$, d'ouverture petite $\sim \pi$, alors la borne $M$ est proche de $T|\alpha|$; si, par contre, l'ouverture de $V$ est grande $\sim 2 \pi$, alors la borne $M$ devient très grande. 
Maintenant, en appliquant la dernière estimation du Lemme 4.31 à $\tilde{g}$, puis en revenant dans la variable $w$, il vient :

$$
\begin{aligned}
\psi_{*} g^{\circ N}(w) & =\left[w^{q / p}+\frac{q}{p} \alpha N+O\left(\frac{N}{|w|^{\varepsilon}}\right)\right]^{p / q} \\
& =w\left[1+\frac{q}{p} \alpha N w^{-q / p}+O\left(\frac{N}{|w|^{\frac{q}{p}+\varepsilon}}\right)\right]^{p / q} \\
& =w+\alpha N w^{1-\frac{q}{p}}+O\left(N|w|^{1-\frac{q}{p}-\varepsilon}\right)+O\left(N^{2}|w|^{1-2 \frac{q}{p}}\right)
\end{aligned}
$$

En substituant $w:=w+n$ et $N:=\left[t \cdot n^{\frac{q}{p}-1}\right]$, il vient :

$$
\begin{aligned}
\psi_{*} g^{\circ\left[t \cdot k_{n}\right]}(w+n)-w-n= & \frac{\left[t \cdot n^{\frac{q}{p}-1}\right]}{n^{\frac{q}{p}-1}} \alpha\left(1+\frac{w}{n}\right)^{1-\frac{q}{p}} \\
& +\frac{1}{n^{\varepsilon}} O\left(\left(1+\frac{|w|}{n}\right)^{1-\frac{q}{p}-\varepsilon}\right) \\
& +\frac{1}{n} O\left(\left(1+\frac{|w|}{n}\right)^{1-2 \frac{q}{p}}\right),
\end{aligned}
$$

ce qui nous donne les estimations finales désirées.

Le lemme 4.28 se déduit immédiatement du Lemme 4.32.

Démonstration du Théorème 4.26. Nous reprenons les notations des sections 2.5 et 3.11 :

$$
G_{k}=\left\{c \in \mathbb{C} ; \exists g(z)=z+c z^{k+1}+\cdots \in G\right\} \quad \text { pour } \quad k \in \mathbb{N}^{*}
$$

et

$$
K=\left\{k \in \mathbb{N}^{*} ; G_{k} \neq\{0\}\right\} .
$$

Supposons d'abord que $G$ ne soit pas conjugué à un sous-groupe de $\operatorname{Diff}(\mathbb{R}, 0)$. D'après le Corollaire 3.42 , on peut trouver des éléments

$$
f(z)=z+c z^{p+1}+\cdots \in G \quad \text { et } \quad g_{i}(z)=z+d_{i} z^{q+1}+\cdots \in G, i=1,2
$$

avec $0<p<q, c, d_{1}, d_{2} \neq 0$ et $d_{2} / d_{1} \notin \mathbb{R}$. En appliquant le Lemme 4.28 à $f$ et $g_{i}, i=1,2$, on déduit dans chaque pétale attractif $V$ de $f$ deux champs de vecteurs $X_{i}=\frac{-p d_{i}}{(-p c)^{q / p}} X, i=1,2$, dont les flots réels sont dans l'adhérence de $G$, où $X$ est le générateur infinitésimal de $f$ sur $V$. Clairement, l'action combinée de ces deux champs est transitive sur $V$; par suite, les orbites de $G$ sont denses sur $V$. En remplaçant $f$ par $f^{\circ(-1)}$ sur les pétales répulsifs, on déduit la densité des orbites sur tous les pétales, i.e. sur un voisinage épointé $U^{*}$ de 0 . 
Supposons maintenant $G$ réel dans une bonne coordonnée analytique. Si $d=\operatorname{pgcd}(K)>1$, on peut supposer d'après le Corollaire 3.41 que $G$ est le relevé par le revêtement ramifié $z \mapsto z^{d}$ d'un sous-groupe $\tilde{G} \subset \operatorname{Diff}(\mathbb{R}, 0)$ pour lequel $\operatorname{pgcd}(\tilde{K})=1$. Pour terminer la preuve du Théorème 4.26, il suffit de montrer que $\tilde{G}$ agit avec orbites denses sur les composantes connexes de $\tilde{\Sigma} \backslash\{0\}$ et $U \backslash \tilde{\Sigma}$ où $\tilde{\Sigma}=\mathbb{R} \cap U$. En effet, on aura alors l'énoncé analogue pour $G$ avec $\Sigma=\left\{z^{d} \in \mathbb{R}\right\}$.

Pour ne pas alourdir inutilement les notations, supposons $G$ réel et $\operatorname{pgcd}(K)=1$. Alors $K$ contient tous les entiers à partir d'un certain rang et on peut trouver dans $G$ des éléments

$$
f_{i}(z)=z+c_{i} z^{p_{i}+1}+\cdots \quad \text { et } \quad g_{i}(z)=z+d_{i} z^{2 p_{i}+1}+\cdots, \quad i=1,2
$$

tels que $0<p_{1}<p_{2}=p_{1}+1$ et $c_{i}, d_{i} \in \mathbb{R}^{*}, i=1,2$. En appliquant le Lemme 4.28 à $f_{i}$ (resp. $f_{i}^{\circ(-1)}$ ) et $g_{i}$, on déduit que, sur chaque pétale de $f_{i}$, le flot réel du générateur infinitésimal $X_{i}=c_{i} z^{p_{i}+1} \partial_{z}+\cdots$ de $f_{i}$ est dans l'adhérence de $G$. Le lieu de tangence entre les flots de $X_{1}=F_{1}(z) \partial_{z}$ et $X_{2}=F_{2}(z) \partial_{z}$ est donné par $F_{2} / F_{1} \in \mathbb{R}$ et est asymptotiquement égal à $z \in \mathbb{R}$ (dans l'intersection des pétales de $f_{1}$ et $f_{2}$ où ces champs sont définis). On déduit que les champs $X_{i}$ agissent localement transitivement en dehors d'une unique courbe réelle dont l'équation, dans chaque pétale, est asymptotique à $\operatorname{Im}(z)=0$. Cette courbe est a priori analytique dans les pétales et différentiable en 0 . Ça ne peut être que $\Sigma=\mathbb{R} \cap U$ qui est elle même évidemment invariante. Enfin, $X_{1}$ agit transitivement sur les deux composantes de $\Sigma \backslash\{0\}$ ce qui termine la preuve.

Corollaire 4.33. Soient $f$ et $g$ deux difféomorphismes tangents à l'identité et soit $\Sigma \subset U^{*}$ une courbe analytique accumulant 0 à la fois invariante par $f$ et $g$. Si $\Sigma$ n'est pas analytique en 0 , alors $f$ et $g$ commutent.

Démonstration. Il suffit de remarquer que si $f$ et $g$ engendrent un groupe non abélien, alors il est non résoluble (voir Théorème 2.22) ce qui contredit l'analyticité des courbes invariantes.

Notons que tout germe tangent à l'identité $f$ admet beaucoup de courbes invariantes qui sont analytiques en dehors de 0 . Pour en construire une, il suffit de se donner une courbe analytique fermée dans une des sphères du chapelet que l'on déroule dans le pétale correspondant. Une telle courbe est analytique en 0 que si, et seulement si, $f$ est conjugué à un germe de difféomorphisme réel (voir section 3.8.4). 


\subsection{Clôture topologique d'un pseudo-groupe et pseudo-algèbre de Lie}

Nous introduisons maintenant le concept de clôture topologique $\bar{G}$ d'un pseudo-groupe général $G$ ainsi que du faisceau $\mathcal{A}$ d'algèbres de Lie associé (généralisant les flots de Nakai) afin de pousser plus loin la description des dynamiques non résolubles. Rappelons d'abord la définition générale d'un pseudo-groupe.

\subsubsection{Pseudo-groupes}

Un pseudo-groupe sur une variété complexe $M$ est une collection

$$
G=\left\{\varphi_{i}: U_{i} \rightarrow V_{i}\right\}_{i \in I}
$$

de difféomorphismes holomorphes $\varphi: U \rightarrow V$ entre ouverts $U, V \subset M$ de $M$ satisfaisant :

- l'identité id : $U \rightarrow U$ appartient à $G$ pour tout ouvert $U \subset M$;

— si $(\varphi: U \rightarrow V)$ appartient à $G$, alors son inverse défini par $\left(\varphi^{-1}\right.$ : $V \rightarrow U)$ appartient aussi à $G$;

— si $(\varphi: U \rightarrow V)$ et $(\psi: V \rightarrow W)$ appartiennent à $G$, alors leur composition $(\psi \circ \varphi: U \rightarrow W)$ appartient aussi à $G$;

— si $(\varphi: U \rightarrow V)$ appartient à $G$, alors ses restrictions à tout ouvert $U^{\prime} \subset U,\left(\left.\varphi\right|_{U^{\prime}}: U^{\prime} \rightarrow \varphi\left(U^{\prime}\right)\right)$, appartiennent aussi à $G$;

- un difféomorphisme $\varphi: U \rightarrow V$ entre ouverts de $M$ appartient à $G$ dès que ses restrictions $\left.\varphi\right|_{U_{j}}: U_{j} \rightarrow \varphi\left(U_{j}\right)$ appartiennent à $G$ pour un recouvrement ouvert $U=\cup_{j \in J} U_{j}$ (non nécessairement fini).

Grosso-modo, la différence principale entre un pseudo-groupe et un groupe est que l'on ne peut composer deux éléments que lorsque l'image du premier coïncide avec le domaine de définition du second : la loi de composition est partielle.

L'orbite d'un point $z_{0} \in M$ par un pseudo-groupe $G$ sur $M$ est l'ensemble des points $z \in M$ tels qu'il existe un élément $\varphi: U \rightarrow V$ dans $M$ avec $z_{0} \in U$ et $\varphi\left(z_{0}\right)=z$.

L'intersection de pseudo-groupes est un pseudo-groupe et on peut parler du pseudo-groupe engendré sur $M$ par une collection de transformations

$$
G=<\varphi_{i}: U_{i} \rightarrow V_{i}>_{i \in I}
$$

(ou encore par plusieurs pseudo-groupes sur $M$ ). On peut aussi restreindre un pseudo-groupe à un ouvert $U \subset M$ en ne considérant que les éléments de $G$ qui agissent dans $U$.

Le pseudo-groupe trivial sur $M$ est la collection des applications identité sur les ouverts $U$ de $M$; c'est le plus petit pseudo-groupe sur $M$. Le plus gros, noté $\operatorname{Diff}(M)$, est le pseudo-groupe de toutes les difféomorphismes 
holomorphes $\varphi: U \rightarrow V$ entre ouverts de $M$; les autres pseudo-groupes sont entre les deux. Par exemple, un groupe $G$ de difféomorphismes holomorphes sur $M$ définit un pseudo-groupe sur $M$ en considérant la restriction de ses éléments à tous les ouverts $U \subset M$.

Le flot d'un champ de vecteurs holomorphe $X$ (non nécessairement complet) sur $M$ définit un pseudo-groupe : à chaque chemin $\gamma:[0,1] \rightarrow \mathbb{C}$, $\gamma(0)=0$, on peut associer le flot $\phi_{X}^{\gamma}: U \rightarrow V$ obtenu en intégrant le champ $X$ le long de $\gamma$. Ici, $U_{\gamma}$ est l'ensemble des points de $M$ pour lesquels on peut effectivement intégrer; pour $\gamma$ suffisamment proche de 0 , le domaine de définition $U_{\gamma}$ est non vide. Les applications $\phi_{X}^{\gamma}$ engendrent un pseudo-groupe sur $M$ qui ne dépend que de $X$. On peut aussi considérer le sous-pseudo-groupe définit par le flot réel de $X$ obtenu en se restreignant aux chemins $\gamma:[0,1] \rightarrow \mathbb{R}$.

Les pseudo-groupes apparaissent naturellement en géométrie différentielle comme collection de difféomorphismes préservant une structure géométrique donnée telles qu'une métrique, une forme différentielle, un tenseur, etc...

On peut associer à un pseudo-groupe $G$ sur $M$ un groupoïde de germes, à savoir la collection $\{\varphi:(M, p) \rightarrow(M, q)\}$ de tous les germes de difféomorphismes entre points $p, q \in M$ induits par les éléments de $G$. Cet objet est fermé pour l'inversion et la composition; inversement, tout groupoïde de germes définit un pseudo-groupe que l'on déduit en recollant les germes. Il est quelque fois moins lourd de travailler avec ce dernier objet, ne serait-ce que pour éviter de trainer les propriétés de restriction et recollement.

\subsubsection{Clôture topologique d'un pseudo-groupe}

Un difféomorphisme $\phi: U \rightarrow V$ entre deux ouverts de $M$ est limite topologique d'une suite de difféomorphismes $\phi_{n}: U \rightarrow V_{n}, n \in \mathbb{N}$, si, pour tout compact $K \subset U$, la suite des applications restreintes $\phi_{n}$ converge uniformément sur $K$ vers $\phi$ lorsque $n \rightarrow \infty$. La clôture topologique $\bar{G}$ d'un pseudo-groupe $G$ sur $M$ est l'adhérence topologique de $G$ au sens précédent. En fait, la collection de toutes les limites possibles de suites d'éléments de $G$ est presque un pseudo-groupe : seul le dernier axiome de recollement n'est pas satisfait. Alors, $\bar{G}$ est par définition le pseudogroupe engendré, c'est-à-dire dont les éléments sont obtenus en recollant ceux obtenus dans l'adhérence de $G$ au sens précédent. On dira que $G$ est fermé lorsque $\bar{G}=G$, et qu'il est discret si toute suite convergente est stationnaire.

On peut munir Diff( $M)$ d'une structure de pseudo-groupe topologique de telle sorte que $\bar{G}$ soit la clôture de $G$ dans $\operatorname{Diff}(M)$ pour cette topologie. Pour cela, on définit d'abord une exhaustion d'un ouvert $U \subset M$ par des compacts comme étant une suite croissante de compacts

$$
K_{0} \subset K_{1} \subset \cdots \subset K_{n} \subset K_{n+1} \subset \cdots \subset U
$$


satisfaisant $U=\cup_{n \in \mathbb{N}} K_{n}$ et, pour tout compact $K \subset U, K \subset K_{n}$ pour $n>>0$. Maintenant, fixons une métrique $d$ sur $M$. Étant donné un élément $\phi: U \rightarrow V$, choisissons des exhaustions de $U, K_{n} \subset U$, et de son complémentaire, $K_{n}^{\prime} \subset M \backslash \bar{U}$, par des compacts puis notons $U_{n}=M \backslash K_{n}^{\prime}$. Alors une base de voisinages de $\phi$ est donnée par

$$
\mathcal{V}_{0}:=\operatorname{Diff}(M) \supset \mathcal{V}_{1} \supset \mathcal{V}_{2} \supset \cdots \supset\{\phi: U \rightarrow V\}
$$

où $\mathcal{V}_{n}(\phi)$ est défini, pour $n \in \mathbb{N}^{*}$, comme l'ensemble

$$
\left\{\phi^{\prime}: U^{\prime} \rightarrow V^{\prime} ; K_{n} \subset U^{\prime} \subset U_{n} \text { et } \sup _{p \in K_{n}} d\left(\phi^{\prime}(p), \phi(p)\right) \leq 1 / n\right\} .
$$

La topologie ainsi engendrée sur Diff $(M)$ est Hausdorff (séparée) et on vérifie aisément que la composition et le passage à l'inverse sont continues.

Notons que deux éléments de $\operatorname{Diff}(M)$ qui se déduisent l'un de l'autre par prolongement analytique sont automatiquement dans la même composante connexe pour cette topologie. En particulier, on peut parler de la composante connexe de l'identité pour n'importe quel pseudo-groupe $G$ sur $M$; par exemple, $G$ est discret si sa composante neutre est triviale.

Remarque 4.34. L'adhérence de l'orbite $\overline{\operatorname{Orb}(z)}$ d'un point z par $G$ contient l'orbite de z sous l'action de $\bar{G}$. En particulier, si le groupe $G$ n'est pas discret, l'orbite générique ne le sera pas non plus. En effet, l'ensemble des points fixés par un élément non trivial de $G$ est dénombrable; si une orbite $\operatorname{Orb}(z)$ ne rencontre pas de tels points, alors l'action de $G$ sur $\operatorname{Orb}(z)$ est "libre" et toute suite $\varphi_{n}: V_{n} \rightarrow U$ de $G$ convergeant vers l'identité va induire une suite $\varphi_{n}(z)$ non stationnaire dans $\operatorname{Orb}(z)$ convergeant vers z. Attention, la réciproque est fausse: les sous-groupes non linéarisables de $\operatorname{Diff}(\mathbb{C}, 0)$ de type $(\operatorname{Lin})^{\mathrm{ex}}$ (voir section 4.1) engendrent des pseudo-groupes discrets dont les orbites ne le sont pas...

\subsubsection{Pseudo-algèbre de Lie d'un pseudo-groupe fermé}

Étant donné un champ de vecteurs holomorphe $X$ défini sur un ouvert $U \subset M$, on peut définir son flot réel $\phi^{t}: U_{t} \rightarrow U$ en intégrant le champ de vecteurs réel sous-jacent. Par exemple, dans le cas $U \subset \mathbb{C}$, le flot réel du champ de vecteurs $X=f(z) \partial_{z}$ sur $U$ est obtenu en intégrant le champ de vecteurs réel Ré $(f(x+i y)) \frac{\partial}{\partial x}-\operatorname{Im}(f(x+i y)) \frac{\partial}{\partial y}$ où $z=x+i y$. En général, le domaine de définition $U_{t}$ de $\phi_{X}^{t}$ est un ouvert strict de $U$ dès que $t \neq 0$ et devient vide pour $|t|>>0$. Un tel flot est continu pour la topologie précédente.

Étant donné un pseudo-groupe $G$ sur $M$, considérons, pour tout ouvert $U \in M$, la collection

$$
\mathcal{A}(U)=\left\{X \in \mathcal{X}(U) ;\left(\phi_{X}^{t}: U_{t} \rightarrow U\right) \in G \text { pour tout } t \in \mathbb{R}\right\}
$$


de champs de vecteurs holomorphes $X$ sur $U \subset M$ dont le flot réel est entièrement contenu dans $G$. Bien sûr, il suffit que le flot aux temps $t \in$ ]$-\varepsilon, \varepsilon[$ soit contenu dans $G$ pour qu'il le soit pour tout $t \in \mathbb{R}$. On peut vérifier que $\mathcal{A}$ est un sous-faisceau du faisceau $\mathcal{X}$ des champs de vecteurs holomorphes sur $M$ et qu'il est invariant sous l'action de $G$ : tout élément $\varphi: U \rightarrow V$ de $G$ induit un isomorphisme par conjugaison

$$
\varphi^{*}: \mathcal{A}(V) \rightarrow \mathcal{A}(U) ; X \mapsto \varphi^{*} X .
$$

C'est un fait classsique que si $G$ est topologiquement fermé, alors $\mathcal{A}$ est un faisceau d'algèbres de Lie réelles (pour le crochet de Lie $[X, Y]$ sur les champs de vecteurs) et est aussi fermée pour la convergence uniforme sur les compacts. Ceci découle des formules

$$
\begin{gathered}
\phi_{s X}^{t}=\phi_{X}^{s t}, \quad \phi_{X+Y}^{t}=\lim _{n \rightarrow+\infty}\left(\phi_{X}^{t / n} \circ \phi_{Y}^{t / n}\right)^{n} \\
\text { et } \phi_{[X, Y]}^{t}=\lim _{n \rightarrow+\infty}\left(\phi_{X}^{t / n} \circ \phi_{Y}^{t / n} \circ \phi_{X}^{-t / n} \circ \phi_{Y}^{-t / n}\right)^{n^{2}}
\end{gathered}
$$

dérivées de la formule de Campbell-Hausdorff à l'ordre 2 :

$$
\exp (X) \circ \exp (Y)=\exp \left(X+Y+\frac{1}{2}[X, Y]+o(X Y)\right) .
$$

L'algèbre de Lie $\mathcal{A}$ ainsi définie n'est bien sûr que réelle comme le montre l'exemple des pseudo-groupes d'isométries de la sphère, du plan Euclidien ou du disque de Poincaré.

Notons $\mathcal{G}$ le pseudo-groupe engendré sur $M$ par les flots réels des éléments de $\mathcal{A}$. Clairement, $\mathcal{G}$ est contenu dans la composante neutre $\bar{G}^{0}$ de $\bar{G}$. Par contre, il se peut que $\mathcal{G} \neq \bar{G}^{0}$, et en particulier que $\bar{G}^{0}$ soit non trivial ( $G$ non discret) et pourtant $\mathcal{G}$ soit réduit à 0 en un point donné. Par action de $\mathcal{A}$ sur $M$, nous entendrons l'action de $\mathcal{G}$.

Remarque 4.35. L'adhérence de l'orbite $\overline{\operatorname{Orb}(z)}$ d'un point z par $G$ contient l'orbite de $z$ sous l'action de $\mathcal{G}$.

Remarque 4.36. Le Théorème de Nakai (ou plutôt le Lemme 4.28) nous dit que l'algèbre de Lie $\mathcal{A}$ du pseudo-groupe induit par un sous-groupe non résoluble $G \subset \operatorname{Diff}(\mathbb{C}, 0)$ est non triviale en tout point d'un voisinage épointé (et même de dimension infinie comme nous allons le voir).

\subsubsection{Pseudo-groupes fermés de dimension infinie en dimension 1}

Là où l'algèbre de Lie $\mathcal{A}$ de $G$ est non triviale, on a une description précise des orbites de $\mathcal{G}$ et donc de $\bar{G}$, et par suite de l'adhérence des 
orbites de $G$ : ce sont localement des sous-variétés analytiques réelles de dimension 0,1 ou 2. Notons que $\mathcal{A}$ est invariante sous l'action de $\mathcal{G}$ et est en ce sens constante le long des orbites. Par ailleurs, puisqu'elle est invariante sous l'action de $G$, ce dernier préserve les orbites de $\mathcal{G}$. Par exemple, lorsque $\mathcal{A}$ est de dimension 1 (réelle), les orbites de $\bar{G}$ sont les feuilles d'un feuilletage analytique réel au voisinage d'un point générique. $\mathrm{Au}$ voisinage d'un point fixe, on est ramené aux modèles de la Proposition 2.3 ; rappelons dans ce cas que les orbites (de dimension 1 réelle) ne sont en général pas analytiques au voisinage des points fixes. Par contre, dès que l'algèbre de Lie est de dimension plus grande (par exemple non abélienne), on a :

Proposition 4.37. Soit $\bar{G}$ un pseudo-groupe fermé sur un ouvert $U$ de $\mathbb{C}$. On suppose que son algèbre de Lie $\mathcal{A}(U)$ est de dimension réelle $\geq 2$ sur $U$. Notons $E \subset U$ l'ensemble discret des points fixes de $\mathcal{G}$. Alors il existe un sous-ensemble analytique réel $\Sigma \subset U$ de dimension 1 tel que

- chaque composante connexe de $U \backslash \Sigma$ est une orbite de dimension 2 pour $\mathcal{G}$,

- chaque composante connexe de $\Sigma-(E \cap \Sigma)$ est une orbite lisse de dimension 1 pour $\mathcal{G}$,

- au voisinage de chaque point fixe $z_{0} \in E \cap \Sigma$, l'ensemble $\Sigma$ est donné dans une coordonnée locale $z$ convenable par $\Sigma=\left\{z^{p} \in \mathbb{R}\right\}$ pour un $p \in \mathbb{N}^{*}\left(\right.$ et $z_{0}$ par $\left.z=0\right)$.

Notons que $G$, quant à lui, permute les orbites de même dimension.

Démonstration. Étant donnés deux éléments $X, Y \in \mathcal{A}(U)$ dont les flots réels sont tangents en $0, X=f(z) \partial_{z}$ et $Y=g(z) \partial_{z}$, alors le lieux de tangence entre ces deux flots sur $U$ est donné par $\left\{\frac{f(z)}{g(z)} \in \mathbb{R}\right\}$. À permutation près de $f$ et $g$, la fonction $\frac{f(z)}{g(z)}$ est holomophe en 0 et prend la forme $z^{p}$ pour un $p \in \mathbb{N}^{*}$ et une coordonnée $z$ convenable. Alors $\Sigma$ se déduit en effaçant de $\left\{z^{p} \in \mathbb{R}\right\}$ les branches non invariantes par $\mathcal{G}$.

Le long d'une orbite ouverte (de dimension 2 réelle), l'algèbre de Lie est décrite localement par le Théorème de Lie : si elle est de dimension finie, c'est une sous-algèbre de Lie réelle d'un des modèles locaux donnés par le Théorème 2.1. Lorsqu'elle est de dimension infinie, on a le :

Proposition 4.38. Soit $G$ un pseudo-groupe sur un ouvert $U$ de $\mathbb{C}$ dont l'algèbre de Lie $\mathcal{A}(U)$ est de dimension infinie. Alors en restriction à toute $\mathcal{G}$-orbite $V$ de dimension $2, \mathcal{A}=\mathcal{X}$ est le faisceau d'algèbres de Lie de tous les champs de vecteurs holomorphes sur $U$ et $\bar{G}=\operatorname{Diff}(V)$ est le pseudo-groupe de toutes les transformations conformes dans $V$.

Le long d'une orbite de dimension 1 , disons $\left(\Sigma, z_{0}\right)=(\mathbb{R}, 0),\left(\mathcal{A}, z_{0}\right)=$ $\mathcal{X}(\mathbb{R}, 0)$ est l'algèbre des germes de champs de vecteurs réels; $\bar{G}$ est engendré par $\operatorname{Diff}(\mathbb{R}, 0)$ et par les translations réelles au voisinage de $\Sigma$. 
Démonstration. Puisque $\mathcal{A}$ est un faisceau localement constant le long de chaque orbite, en particulier celles de dimension 2, il suffit de montrer que $(\mathcal{A}, p)=(\mathcal{X}, p)$ au voisinage d'un point $p$ de cette orbite, disons $p=0$ pour simplifier. Par hypothèse, il existe au moins 3 éléments de $(\mathcal{A}, 0)$ de la forme

$X=\partial_{z}+\cdots, \quad Y=i \partial_{z}+\cdots \quad$ et $\quad Z=\alpha z^{p+1} \partial_{z}+\cdots, \alpha \in \mathbb{C}^{*}, p>>0$

( $X$ et $Y$ pour la transitivité et $Z$ pour la dimension infinie). Alors il vient

$$
[X, Z]=\alpha z^{p} \partial_{z}+\cdots \quad \text { et } \quad[Y, Z]=i \alpha z^{p} \partial_{z}+\cdots ;
$$

ainsi, pour tout $c \in \mathbb{C}^{*},(\mathcal{A}, 0)$ contient un élément de le forme $c z^{p} \partial_{z}+\cdots$. En réitérant l'argument précédent, on déduit, pour tout $c \in \mathbb{C}^{*}$ et tout $k=0,1, \ldots, p$, un élément de la forme $c z^{k} \partial_{z}+\cdots \in(\mathcal{A}, 0) ;$ puisque $p$ peut être choisi arbitrairement grand, l'assertion précédente vaut pour tout $k \in \mathbb{N}$. En particulier, $(\mathcal{A}, 0)$ contient un élément de la forme $i z \partial_{z}+\cdots$ qui se linéarise dans une bonne coordonnée; alors $\bar{G}$ contient son flot et en particulier la contraction $\varphi(z)=z / 2$ sur un disque $\mathbb{D}_{\varepsilon}$ centré en 0 . Étant donné un élément $X=\alpha z^{k} \partial_{z} \cdots$ de $\mathcal{A}$, la suite d'éléments $X_{n}:=2^{n(k-1)}\left(\varphi^{\circ n}\right)^{*} X$ est bien définie sur $\mathbb{D}_{\varepsilon}$ pour $n>>0$ suffisamment grand et tend vers le champ de vecteurs monomial $X_{\infty}=\alpha z^{k} \partial_{z}$ sur $\mathbb{D}_{\varepsilon}$. Puisque $\mathcal{A}\left(\mathbb{D}_{\varepsilon}\right)$ est fermée par convergence uniforme, elle contient tous les champs de vecteurs monomiaux $\alpha z^{k} \partial_{z}$, et par suite, tous les champs de vecteurs holomorphes $\sum_{k=0}^{\infty} \alpha_{k} z^{k} \partial_{z}$ qui convergent sur $\mathbb{D}_{\varepsilon}$. De la même manière, tout champ de vecteurs défini sur un disque plus petit est aussi uniformément approché par les éléments de $\mathcal{A}$ et donc appartient à $(\mathcal{A}, 0)$.

Lorsque l'on est sur $\Sigma=\mathbb{R}$, c'est la même preuve en remarquant préalablement que $(\mathcal{A}, 0) \subset \mathcal{X}(\mathbb{R}, 0)$.

Démonstration du Théorème 4.27. Au vu du Théorème 4.26 et de la Proposition précédente, il suffit de remarquer que l'algèbre de Lie $\left(\mathcal{A}, z_{0}\right)$ est de dimension infinie en tout point $z_{0} \in U^{*}$ suffisamment proche de 0 . On reprend les notations de la section 4.3. Quitte à diminuer $U$, tout point $z_{0} \in U$ est contenu à la fois dans un pétale $V$ de $f$ et un pétale $W$ de $g$. Soient $X$ et $Y$ les générateurs infinitésimaux respectifs de $f$ et $g$ sur ces pétales. Le lemme de Nakai permet de construire le flot réel de $\alpha X$ au voisinage de $z_{0}$ comme limite uniforme d'éléments de $G$. Maintenant, en réappliquant le lemme de Nakai à $g(z)=z+d z^{q+1}+\cdots$ et $[f, g](z)=z+c d(q-p) z^{p+q+1}+\cdots$, on récupère le flot réel de $\beta Y$ au voisinage de $z_{0}$. L'algèbre de Lie engendrée par $\alpha X$ et $\beta Y$ est bien sûr de dimension infinie puisque l'algèbre de Lie formelle engendrée par les développements asymptotiques de $X$ et $Y$ à l'origine l'est.

Nous déduisons comme corollaire le résultat de [10] : 
Corollaire 4.39. Soit $G$ le pseudo-groupe induit sur $U$ par un sous-groupe non résoluble de $\operatorname{Diff}(\mathbb{C}, 0)$. Alors l'ensemble des points fixes attractifs

$$
\left\{z \in U ; \exists g \in G, g(z)=z \text { et }\left|g^{\prime}(z)\right|<1\right\}
$$

est dense dans $U$. En particulier, la seule mesure de probabilité sur $U$ qui soit invariante par $G$ est la masse de Dirac en 0 .

Démonstration. En tout point $z_{0} \in U^{*}$, la contraction affine $\varphi(z)=z_{0}+$ $\frac{z-z_{0}}{2}$ est approchée uniformément sur un voisinage $V$ de $z_{0}$ par une suite d'éléments $g_{n}$ de $G$. Pour $n>>, g_{n}$ admet un point fixe $z_{n}$ dans $U$ d'après le Théorème de Rouché et sa dérivée $g_{n}^{\prime}\left(z_{n}\right)$ est proche de $1 / 2$ ce qui démontre la première assertion. On peut même choisir un recouvrement de $U^{*}$ par de tels $V$ de sorte que tout point de $U^{*}$ se trouve dans le bassin d'attraction d'une contraction de $G$. Si le support d'une mesure $G$-invariante intersecte $U^{*}$, alors la mesure va charger au moins un point fixe attractif; comme son orbite sous $G$ est infinie (Théorème de Nakai), la mesure est infinie.

Des résultats de Shcherbakov (Théorème 4.25), il nous reste à démontrer que toute conjugaison topologique entre dynamiques résolubles est conforme.

Démonstration du Théorème 4.25, (2). Soit $\psi: U \rightarrow \mathbb{C}$ un homéomorphisme conjugant $G$ à un pseudo-groupe conforme $\psi_{*} G=\tilde{G}$.

Montrons d'abord que $\psi$ est différentiable sur les secteurs de Nakai. En effet, $\psi$ conjugue les adhérences respectives de $G$ et de $\tilde{G}$, et par suite les algèbres de Lie associées $\mathcal{A}$ et $\tilde{\mathcal{A}}$. En un point $z_{0} \in U \backslash \Sigma$, on peut trouver deux champs de vecteurs $X, Y \in \mathcal{A}$ transverses paramétrant le voisinage de $z_{0}$ par

$$
\phi:\left(\mathbb{R}^{2}, 0\right) \rightarrow\left(U, z_{0}\right) ;(t, s) \mapsto \phi_{X}^{t} \circ \phi_{Y}^{s}\left(z_{0}\right) .
$$

Les images $\tilde{X}$ et $\tilde{Y}$ par $\psi$ permettent de paramétrer le voisinage de $\psi\left(z_{0}\right)$ par une application différentiable $\tilde{\phi}$; par construction, $\psi=\tilde{\phi} \circ \phi^{\circ(-1)}$ est différentiable en $z_{0}$.

Montrons maintenant que $\psi$ est holomorphe sur les secteurs de Nakai ; elle sera alors holomorphe partout. Si l'on tire en arrière par $\psi$ la structure conforme standart, invariante par $\tilde{G}$, on obtient une structure quasiconforme sur $U \backslash \Sigma$ invariante par $G$. Elle est par exemple donnée par un champ d'ellipses différentiable. Si c'est la structure conforme standart, alors $\psi$ est conforme sur $U \backslash \Sigma$, ce que nous voulons démontrer. Sinon, alors les ellipses ne sont pas des cercles et leur rayon maximal induit un champ de droites réelles invariant par $G$. Par un argument similaire à la preuve du Corollaire 4.39, l'ensemble

$$
\left\{z \in U ; \exists g \in G, g(z)=z \text { et } g^{\prime}(z) \notin \mathbb{R}\right\}
$$


est dense dans $U \backslash \Sigma$ ce qui contredit l'existence d'un champ de droites invariant (de support non vide).

Exemple 4.40. Comme nous l'avons vu dans la section 2.6, le sous-groupe $G \subset \operatorname{Diff}(\mathbb{C}, 0)$ engendré par

$$
f(z)=z /(1-z) \quad \text { et } \quad g(z)=z /\left(1-2 z^{2}\right)^{1 / 2}
$$

est libre de rang 2. En particulier, il est non résoluble et, d'après le Théoréme 4.27, son algèbre de Lie $\mathcal{A}$ est de dimension infinie en tout point du voisinage épointé $U^{*}$. Par ailleurs, $f, g$, leurs inverses et en fait tous les éléments de $G$ sont des séries à coefficients entiers, i.e. appartiennent à $\mathbb{Z}[[z]]$. En particulier, aucun flot ne peut être approché par les éléments de $G$ au voisinage de 0 et l'algèbre de Lie $\mathcal{A}$ est triviale en 0 . On peut montrer (voir [124], p.160) que $G$ n'est pas discret en 0 : il existe une suite d'éléments $g_{n} \in G$ définis sur un même voisinage de 0 et convergeant uniformément vers l'identité sur ce voisinage. En fait, le contact de $g_{n}$ à l'identité tend vers l'infini lorsque $n \rightarrow \infty$.

\subsection{Clôture de Malgrange}

Les dynamiques (Lin), (Eucl) et (Aff) décrites dans la section 4.1 sont des exemples de pseudo-groupes admettant une structure géométrique invariante : ils laissent invariante une forme différentielle $\omega$ ou la multiplient par une constante; dans la coordonnée $w=\int \omega$, la dynamique devient une dynamique de translations ou de transformations affines de la droite. Nous avons traité le cas linéaire différemment du cas euclidien en préférant la coordonnée donnée par $z=e^{w}$ qui a le mérite d'être régulière en 0 . Dans les autres cas, la structure géométrique donnée par la coordonnée $w$ n'est pas définie en 0 puisque $\omega$ y a un pôle; elle peut même être multiforme, i.e. avoir une monodromie non triviale autour de 0, dans le cas (Eucl) (voir Remarque 4.16). Comme nous le verrons dans la seconde partie, la présence d'une intégrale première explicite (quadrature de Liouville) pour le feuilletage entraine l'existence d'une structure géométrique invariante pour son pseudo-groupe transverse, définie sur un ouvert de Zariski. Cependant, les intégrales premières construites dans l'exemple 4.19 ne conduisent pas à des intégrales premières raisonables : leur croissance est trop forte et ne peuvent provenir d'une quadrature de Liouville. Dans [127, 128], Bernard Malgrange propose une définition de groupoïde de Galois d'un feuilletage s'inspirant de la Théorie de Galois Différentielle classique élaborée par Kolchin pour les équations différentielles linéaires, notamment du théorème de Schlesinger reliant le groupe de Galois à la clôture de Zariski du groupe de monodromie dans le cas régulier. Ce groupoïde apparaît grosso-modo comme la clôture de Zariski du pseudo-groupe d'holonomie du feuilletage 
pour une topologie mixte analytique/algébrique définie sur les espaces de jets sur lesquels le pseudo-groupe agit. Le groupoïde obtenu est comparable au groupe de Galois de l'équation différentielle non linéaire définie précédemment par Umemura dans un cadre plus général dans [222].

De l'étude menée par Guy Casale dans [48] pour les feuilletages de codimension 1 , il ressort que le feuilletage admet une intégrale première explicite (en un sens un peu plus général que la quadrature de Liouville) si, et seulement si, le groupoïde défini par Malgrange est de dimension transverse finie $\leq 3$; sinon, le groupoïde est maximal (de dimension transverse infinie) égal au pseudo-groupe de toute les transformations holomorphes préservant le feuilletage.

Par restriction aux transversales, le groupoïde de Malgrange définit encore un pseudo-groupe fermé pour la même topologie qui contient en particulier la clôture du pseudo-groupe transverse. La description locale des pseudo-groupes de dimension 1 fermés pour la topologie de Malgrange est faite dans [47]. Le but de cette section n'est certainement pas de refaire ce qui est déjà très bien écrit dans [47], mais d'exposer les résultats en donnant des idées de démonstration sans vraiment donner la définition de Malgrange qui dépasse le cadre de notre exposé. Nous nous contenterons d'une idée de définition et de quelques propriétés qui nous permettront, à moindre mal, de retrouver la classification de [47].

On appellera pseudo-groupe de Lie sur un ouvert $U \subset \mathbb{C}$ tout pseudogroupe $G$ qui est localement défini par des équations différentielles de la forme suivante. Pour toute coordonnée locale $(z, w)$ sur $V \times W$, les éléments de $G$ sont les solutions locales $w=\varphi(z)$ d'une collection d'équations différentielles de la forme

$$
f\left(z, w, w^{\prime}, w^{\prime \prime}, \ldots, w^{(n)}\right)=0 \quad \text { où } \quad P \in \mathcal{O}_{V \times W}\left[w_{1}, \ldots, w_{n}\right] .
$$

Dans [127, 47], l'ensemble de ces équations forme un faisceau d'idéaux $\mathcal{I}_{n}$ sur chaque espace de jets $\mathcal{O}_{V \times w}\left[w_{1}, \ldots, w_{n}, \ldots\right]$ satisfaisant en outre un certain nombre de propriétés $\left(\mathcal{I}_{n}\right.$ est cohérent, réduit, différentiel,...) qui assureront par exemple que l'intersection de pseudo-groupes de Lie est encore un pseudo-groupe de Lie, etc... Maintenant, un pseudo-groupe $G$ (non nécessairement de Lie) sur $U$ étant donné, sa clôture $\bar{G}^{\text {Lie }}$ est le plus petit pseudo-groupe de Lie sur $U$ contenant $G$. De cette définition, nous retiendrons :

le pseudo-groupe $\bar{G}^{\text {Lie }}$ contient toutes les transformations locales $w=$ $\varphi(z)$ dans $U$ qui satisfont à toutes les équations différentielles

$$
f\left(z, w, w^{\prime}, w^{\prime \prime}, \ldots, w^{(n)}\right)=0
$$

satisfaites par les éléments de $G$.

Proposition 4.41. Tout pseudo-groupe de Lie G sur U est fermé pour la topologie de convergence uniforme définie dans la section 4.4.2. 
Ainsi, la clôture de Lie $\bar{G}^{\text {Lie }}$ d'un pseudo-groupe $G$ contient notamment sa clôture topologique $\bar{G}$.

Démonstration. Soit une suite $\varphi_{n}: V \rightarrow U$ d'éléments de $G$ tendant vers $\varphi: V \rightarrow U$ sur tout compact. Pour chaque équation différentielle définissant $G, \varphi_{n}$ est solution et donc $\varphi$ aussi.

Corollaire 4.42. Soit $G \subset \operatorname{Diff}(\mathbb{C}, 0)$ un sous-groupe non résoluble et notons encore $G$ le pseudo-groupe induit sur un voisinage $U$ de 0 par un système de générateurs. Alors, quitte à diminuer $U$, on a $\bar{G}^{\text {Lie }}=\operatorname{Diff}(U)$, le pseudo-groupe de toutes les transformations conformes dans $U$.

Démonstration. Puisque $\bar{G}$ contient $\operatorname{Diff}(V)$ pour chaque secteur de Nakai $V$, tous ses éléments ne peuvent satisfaire simultanément une équation différentielle méromorphe $f\left(z, w, w^{\prime}, w^{\prime \prime}, \ldots, w^{(n)}\right)=0$ non triviale au voisinage de 0 .

L'argument précédent s'applique aussi bien pour tout pseudo-groupe en dimension 1 dont l'algèbre de Lie est de dimension infinie.

Il est intéressant de voir $\bar{G}^{\text {Lie }}$ comme limite inductive des clôtures de Zariski des relevés de $G$ dans les espaces de jets. Considérons le pseudogroupe Diff $(U)$ de tous les difféomorphismes entre ouverts de $U$ et notons $J^{\infty} \operatorname{Diff}(U)$ le groupoïde de germes induit

$$
J^{\infty} \operatorname{Diff}(U)=\{\varphi:(U, z) \rightarrow(U, w) ; z, w \in U \text { avec } \varphi \text { inversible }\} .
$$

Cet ensemble peut être vu comme un fibré analytique localement trivial sur $U \times U$ (donné par la projection $(\varphi:(U, z) \rightarrow(U, w)) \mapsto(z, w))$ de fibre Diff $(\mathbb{C}, 0)$. En effet, dans des coordonnées locales $(z, w)$ sur $V \times W$, ce fibré est localement défini par

$$
J^{\infty} \operatorname{Diff}(V, W)=\{\varphi(z+t)=w+\widetilde{\varphi}(t) ; \widetilde{\varphi} \in \operatorname{Diff}(\mathbb{C}, 0)\} .
$$

On définit l'espace $J^{k} \operatorname{Diff}(U)$ des jets d'ordre $k \in \mathbb{N}$ comme étant l'ensemble des classes d'équivalence pour la relation qui identifie les germes $(\mathbb{C}, z) \rightarrow(\mathbb{C}, w)$ qui coïncident à l'ordre $k$; dans les coordonnées locales, $J^{k} \operatorname{Diff}(V, W)$ est donné par

$$
\left\{w+\sum_{l=1}^{k} a_{l}(z+t)^{l} ;(z, w) \in V \times W, a_{l} \in \mathbb{C}, a_{1} \neq 0\right\} .
$$

Aussi, $J^{k}$ Diff est un fibré localement trivial sur $U \times U$ dont la fibre est le groupe algébrique $J^{k} \operatorname{Diff}(\mathbb{C}, 0)$ de dimension $k$. On a les projections canoniques $\varphi \mapsto J^{k} \varphi$

$$
J^{\infty} \operatorname{Diff}(U) \rightarrow J^{k+1} \operatorname{Diff}(U) \rightarrow J^{k} \operatorname{Diff}(U) \rightarrow J^{0} \operatorname{Diff}(U)=U \times U .
$$


De plus, tout $J^{k} \operatorname{Diff}(U)$ est équipé de la structure de groupoïde de $J^{\infty} \operatorname{Diff}(U)$ : pour tout $\varphi, \psi \in J^{\infty} \operatorname{Diff}(U)$, on a $\left(J^{k} \varphi\right) \circ\left(J^{k} \psi\right):=J^{k}(\varphi \circ \psi)$, dès que la composition $\varphi \circ \psi$ a un sens. Tout pseudo-groupe $G$ sur $U$ induit un sous-groupoïde $J^{k} G \subset J^{k} \operatorname{Diff}(U)$ pour tous $k \in \mathbb{N} \cup\{\infty\}$. Les équations différentielles définissant les pseudo-groupes de Lie se relèvent comme fonctions sur les jets d'ordre suffisamment élevé, i.e. des intégrales premières pour le relèvement naturel du pseudo-groupe. Plus précisément, les fonctions obtenues $\mathcal{O}_{J^{k} \operatorname{Diff}(U)}=\mathcal{O}_{U \times U}\left[a_{1}, \ldots, a_{k}\right]$ sont analytiques dans la direction horizontale et polynomiales dans les fibres. De ce point de vue, la clôture $\bar{G}^{\text {Lie }}$ d'un pseudo-groupe $G$ sur $U$ contient tous les difféomorphismes $\varphi \in \operatorname{Diff}(U)$ dont les jets $J^{k} \varphi$ sont dans la clôture de Zariski de $J^{k} G$ (pour la classe de fonctions $\mathcal{O}_{J^{k} \operatorname{Diff}(U)}$ ci-dessus) pour tous $k>>0$ suffisamment grands.

Des propriétés sur les idéaux différentiels $\mathcal{I}_{n}$ définissant le pseudo-groupe de Lie $G$, on montre que son algèbre de Lie $\mathcal{A}$ est définie par des équations différentielles méromorphes linéaires (voir [127] ou [47]). C'est une algèbre de Lie de champs de vecteurs sur $U$ comparable à celle définie dans la section 4.4.3, à ceci près qu'elle est toujours complexe. Dès qu'elle n'est pas triviale, elle est transitive et donc homogène sur un ouvert de Zariski (complexe) de $U$. Sa dimension est donc $0,1,2$ ou 3 d'après le Théorème de Lie.

Exemple 4.43. Le pseudo-groupe $G$ des transformations qui laissent invariante une fonction méromorphe $f: U \rightarrow \overline{\mathbb{C}}$ est un pseudo-groupe de Lie défini par l'équation différentielle d'ordre 0

$$
f(\varphi)-f(z)=0 .
$$

Guy Casale montre dans [47] que tout pseudo-groupe de dimension 0 est localement de cette forme.

Exemple 4.44. Le pseudo-groupe $G$ des transformations qui laissent invariante une 1-forme méromorphe $\omega$ sur $U$ est un pseudo-groupe de Lie de dimension 1. Écrivons $\omega=\alpha(z) d z$ (dans une coordonnée locale $z$ ) et notons que les éléments de $G$ sont les solutions de l'équation différentielle $\varphi^{*} \omega=\omega$, i.e.

$$
\alpha(\varphi) \varphi^{\prime}-\alpha(z)=0 .
$$

Dans la variable $w=\psi(z)=\int_{0}^{z} \alpha(\zeta) d \zeta$, définie localement au moins en dehors des pôles de $\alpha$, la dynamique de $G$ se redresse sur la dynamique des translations et on retrouve l'équation d'Abel :

$$
\psi \circ \varphi=\psi+t, \quad t \in \mathbb{C} .
$$

En particulier, les modèles locaux abéliens de la section 4.1

$$
\text { (Lin) } \quad\left\{\varphi(z)=a z ; a \in \mathbb{C}^{*}\right\}
$$


et

$$
\text { (Eucl) }\left\{\varphi=a \exp \left(t X_{p, \lambda}\right) ; t \in \mathbb{C}^{*}, a^{p}=1\right\}, \quad p \in \mathbb{N}^{*}, \lambda \in \mathbb{C},
$$

sont des exemples de pseudo-groupes de Lie de dimension 1 au voisinage de 0 . Au voisinage d'un zéro de $\omega$, disons $\omega=d\left(z^{p}\right), p>1$, le pseudo-groupe $G$ est donné par

$$
\left\{\varphi(z)=a\left(z^{p}+t\right)^{1 / p} ; t \in \mathbb{C}^{*}, a^{p}=1\right\} ;
$$

notons alors que les seuls éléments de $G$ définis en 0 sont les rotations $\varphi(z)=a z, a^{p}=1$, et la dimension de $G$ chute en 0 .

Remarque 4.45. En tirant en arrière le pseudo-groupe de translations $w \mapsto w+t$ par l'application $w=\psi(z)=z^{\lambda}$ avec $\lambda \in \mathbb{C}^{*}$, on définit un pseudo-groupe $G$ sur $U^{*}$ : c'est le pseudo-groupe des transformations qui laissent invariante l'une ou l'autre des déterminations de la 1-forme $\omega=d \psi=\lambda z^{\lambda-1} d z$, ou encore c'est le flot complexe du champ de vecteurs $X=\frac{1}{z^{\lambda-1}} \partial_{z}$. En ce sens, $G$ est un pseudo-groupe de Lie de dimension 1 sur $U^{*}$. En général, $G$ ne se prolonge pas en un pseudo-groupe de Lie au sens de Malgrange en 0 . En fait, l'équation définissant $G$

$$
(\varphi)^{\lambda-1} \varphi^{\prime}=z^{\lambda-1}
$$

n'est méromorphe ni en $\varphi$, ni en $z$; cependant, pour $\lambda=\frac{p}{q} \in \mathbb{Q}^{*}$, on récupère une équation méromorphe en élevant la dernière à la puissance $q:$

$$
\alpha(\varphi)\left(\varphi^{\prime}\right)^{q}-\alpha(z)=0, \quad q \in \mathbb{N}^{*}
$$

où $\alpha(z)=z^{p-q}$. En fait, l'équation précédente avec $\alpha$ méromorphe quelconque définit un pseudo-groupe de Lie G pour lequel la coordonnée euclidienne $\psi$ est de la forme $\psi(z)=z^{p / q} u(z)$ où $u(z)$ est holomorphe avec $u(0)=1$ dès que $q>1$; il est alors facile de voir que $\psi=z^{p / q}$ après changement de coordonnée conforme et le pseudo-groupe $G$ est celui précédemment construit.

Exemple 4.46. Le pseudo-groupe $G$ des transformations qui préservent le $\mathbb{C}$-espace vectoriel engendré par une 1-forme méromorphe $\omega$ sur $U$ est un pseudo-groupe de Lie. En effet, les éléments de $G$ satisfont $\varphi^{*} \omega=a_{\varphi} \omega$, $a_{\varphi} \in \mathbb{C}^{*}$; en passant à la dérivée logarithmique, on récupère l'équation différentielle

$$
\beta(\varphi) \varphi^{\prime}+\frac{\varphi^{\prime \prime}}{\varphi^{\prime}}-\beta(z)=0
$$

où $\beta=\frac{\alpha^{\prime}}{\alpha}, \omega=\alpha(z) d z$. Dans la coordonnée $w=\psi(z)=\int_{0}^{z} \alpha(\zeta) d \zeta$, le pseudo-groupe $G$ s'identifie au pseudo-groupe des transformations affines :

$$
\psi \circ \varphi=a \psi+b, \quad a \in \mathbb{C}^{*}, b \in \mathbb{C} .
$$


On retrouve en particulier les modèles locaux de la section 4.1

(Aff) $\quad\left\{\varphi(z)=a z /\left(1-b z^{p}\right)^{1 / p} ; a \in \mathbb{C}^{*}, b \in \mathbb{C}\right\}, \quad p \in \mathbb{N}^{*}$,

au voisinage des points où $\alpha$ a un pôle multiple sans résidu. Là où $\omega$ a un pôle simple, disons $\omega=\frac{d z}{z}$, les translations (de la structure affine) sont données par les transformations linéaires $\varphi(z)=a z, a \in \mathbb{C}^{*}$, et aucun autre élément du pseudo-groupe n'est défini en 0 . En un pôle plus général de $\omega$, seules les translations sont bien définies. En un zéro de $\omega$, le pseudogroupe se réduit à un groupe fini de rotations.

L'équation différentielle (4.4) définit encore un pseudo-groupe de Lie lorsque $\beta$ est une fonction méromorphe quelconque. Là où $\beta$ est holomorphe non nulle, la fonction $\alpha(z)=e^{\int_{0}^{z} \beta(\zeta) d \zeta}$ l'est aussi et la coordonnée $w=$ $\psi(z)=\int_{0}^{z} \alpha(\zeta) d \zeta$ redresse $G$ sur le pseudo-groupe des transformations affines. Les singularités sont cependant plus compliquées que dans le cas où $\alpha$ est méromorphe. En général, aucun élément du pseudo-groupe ne se prolonge en 0 .

Remarque 4.47. Reprenons l'application $\psi(z)=z^{\lambda}, \lambda \in \mathbb{C}^{*}$, de la remarque 4.45 et tirons en arrière, plutôt que les translations, tout le pseudogroupe affine. On obtient cette fois-ci un pseudo-groupe de Lie dont l'équation est (4.4) avec $\beta=(\lambda-1) \frac{d z}{z}$. Pour $\lambda \notin \mathbb{Q}$, c'est la clôture de Lie du pseudo-groupe $G$ considéré dans la remarque 4.45 .

Exemple 4.48. Un pseudo-groupe de Lie $G$ de dimension 3 se redresse, au voisinage d'un point générique, par une transformation conforme $\psi$, sur le pseudo-groupe des transformations de Mobius : tout élément $\varphi$ de $G$ satisfait

$$
\psi \circ \varphi=\frac{a \psi+b}{c \psi+d}, \quad a, b, c, d \in \mathbb{C}, a d-b c \neq 0 .
$$

Considérons la dérivée Schwarzienne $\mathcal{S}$ définie par:

$$
\mathcal{S} f=\left(\frac{f^{\prime \prime}}{f^{\prime}}\right)^{\prime}-\frac{1}{2}\left(\frac{f^{\prime \prime}}{f^{\prime}}\right)^{2}=\frac{f^{\prime \prime \prime}}{f^{\prime}}-\frac{3}{2}\left(\frac{f^{\prime \prime}}{f^{\prime}}\right)^{2} .
$$

Rappelons que $\mathcal{S} f=0$ si, et seulement si, $f$ est une transformation de Mobius et que

$$
\mathcal{S}(f \circ g)=(\mathcal{S} f) \circ g \cdot\left(g^{\prime}\right)^{2}+\mathcal{S} g \quad \text { pour toutes fonctions } f, g \in \mathcal{O}(U)
$$

Alors le pseudo-groupe $G$ est solution de l'équation différentielle $\mathcal{S}(\psi \circ \varphi)=$ $\mathcal{S} \psi$, i.e.

$$
\gamma(\varphi)\left(\varphi^{\prime}\right)^{2}+\frac{\varphi^{\prime \prime \prime}}{\varphi^{\prime}}-\frac{3}{2}\left(\frac{\varphi^{\prime}}{\varphi}\right)^{2}-\gamma(z)=0
$$


où $\gamma=\mathcal{S} \psi$. Réciproquement, toute équation différentielle de la forme précédente avec $\gamma$ méromorphe définit un pseudo-groupe de Lie de dimension 3. La coordonnée projective $\psi$ est donnée par les 3 intégrations successives

$$
\beta^{\prime}-\frac{1}{2} \beta^{2}=\gamma, \quad \frac{\alpha^{\prime}}{\alpha}=\beta \quad \text { et } \quad \psi^{\prime}=\alpha .
$$

Proposition 4.49. Soit $G$ un sous-groupe d'un des groupes de Lie $\mathbb{L}, \mathbb{E}_{p, \lambda}$ ou $\mathbb{A}_{p}$ de la section 4.1. Notons encore $G$ le pseudo-groupe induit par un système de générateurs sur un voisinage $U$ de 0 . Alors sa clôture de Lie $\bar{G}^{\text {Lie }}$ est (le pseudo-groupe induit par) la clôture de Zariski complexe de $G$ dans le groupe de Lie correspondant.

Démonstration. Puisque ces groupes de Lie définissent des pseudo-groupes de Lie au sens de Malgrange, ils contiennent certainement $\bar{G}^{\text {Lie }}$. Dans $\mathbb{L} \simeq \mathbb{C}^{*}$, les sous-groupes Zariski fermés sont les sous-groupes finis et $\mathbb{L}$ luimême. Si $G$ ne contient que des éléments périodiques, alors il est d'ordre fini ou sa clôture topologique $\bar{G}$ contient un élément d'ordre infini. Maintenant, supposons que $\bar{G}^{L i e}$ contienne un élément d'ordre infini $\varphi(z)=e^{2 i \pi t_{0}} z$, $t_{0} \notin \mathbb{Q}$ (et toutes ses itérées); en substituant le groupe à 1 paramètre $\varphi_{t}(z)=e^{2 i \pi t} z$ à n'importe quelle équation différentielle définissant $\bar{G}^{\text {Lie }}$, on obtient une série formelle $f\left(z, \varphi, \varphi^{\prime}, \ldots, \varphi^{(k)}\right)=\sum_{n \geq 0} P_{n}\left(e^{2 i \pi t}\right) z^{n}$ dont les coefficients sont des polynômes en $e^{2 i \pi t}$; par hypothèse, pour chaque $n, P_{n}\left(e^{2 i \pi t}\right)$ s'annule pour tout $t \in t_{0} \mathbb{Z}$ et est donc identiquement nul. Par suite, $\bar{G}^{\text {Lie }}$ contient le groupe à 1 paramètre $\varphi_{t}(z)=e^{2 i \pi t} z$, c'est-à-dire $\mathbb{L}$.

Dans $\mathbb{E}_{p, \lambda}$ ou dans $\mathbb{A}_{p}$, il suffit de montrer que, de la même manière, si $\bar{G}^{\text {Lie }}$ contient un élément de la forme $\varphi=\exp \left(X_{p, \lambda}\right)$, alors $\bar{G}^{\text {Lie }}$ contient le groupe à 1 paramètre $\varphi_{t}=\exp \left(t X_{p, \lambda}\right)$. De nouveau, en substituant $\varphi^{t}=$ $\sum_{n>0} a_{n}(t) z^{n}$ à n'importe quelle équation différentielle définissant $\bar{G}^{\text {Lie }}$, on obtient une série formelle $f\left(z, \varphi, \varphi^{\prime}, \ldots, \varphi^{(k)}\right)=\sum_{n>0} P_{n}\left(a_{1}, \ldots, a_{n}\right) z^{n}$ dont les coefficients $P_{n}\left(a_{1}(t), \ldots, a_{n}(t)\right)$ sont des polynômes en $t$. Ces polynômes s'annulent pour tout $t \in \mathbb{Z}$ et sont donc identiquement nuls. Par suite, le groupe à 1 paramètre $\exp \left(t X_{p, \lambda}\right)$ est entièrement contenu dans $\bar{G}^{\text {Lie }}$.

Nous renvoyons à [47] pour la démonstration du :

Théorème 4.50 (Casale). Tout pseudo-groupe de Lie $G=\bar{G}^{\text {Lie }}$ au sens de Malgrange est, au voisinage $U$ de $0 \in \mathbb{C}$, d'un des types précédents, c'est-à-dire défini par une des équations méromorphes (4.1), (4.3), (4.4) ou (4.5).

On trouve dans [220] le résultat suivant : 
Proposition 4.51 (Touzet). Soit $G \subset \operatorname{Diff}(\mathbb{C}, 0)$ un sous-groupe formellement linéarisable mais non analytiquement linéarisable. Alors la clôture de Lie $\bar{G}^{\text {Lie }}$ du pseudo-groupe induit sur un voisinage $U$ de 0 par un système de générateurs est $\bar{G}^{\text {Lie }}=\operatorname{Diff}(U)$, le pseudo-groupe de toutes les transformations conformes dans $U$.

Démonstration. Supposons par l'absurde que $\bar{G}^{\text {Lie }}$ soit de dimension finie. Alors il existe une coordonnée $\psi: U^{*} \rightarrow \overline{\mathbb{C}}$ multiforme redressant la dynamique de $G$ sur celle des transformations de Møebius. Cela résulte par exemple du théorème précédent. Si $f$ est un élément non périodique de $G$, alors il est non linéarisable d'après la section 4.3. La dynamique de $\psi_{*} f$ commute à la monodromie de $\psi$, car sinon, $f$ serait multiforme autour de 0 . Cependant, aucune orbite de $\overline{\mathbb{C}}$ sous l'action de deux transformations de Mœbius commutantes ne peut avoir une adhérence conformément équivalente au hérisson défini par $f$ près de 0 (voir section 4.3).

Le résultat le plus intéressant obtenu avec l'approche de Malgrange est certainement le suivant.

Théorème 4.52 (Casale). Soit $G$ le pseudo-groupe défini sur un voisinage $U$ de 0 par un difféomorphisme $f(z)=z+\cdots \in \operatorname{Diff}(\mathbb{C}, 0)$ tangent à l'identité. Alors sa clôture de Lie est $\bar{G}^{\text {Lie }}=\operatorname{Diff}(U)$ excepté dans les cas suivants :

- Les invariants analytiques de f sont linéaires

$$
\varphi_{k}^{0}(\tau)=a_{k}^{0} \tau \quad \text { et } \quad \varphi_{k}^{\infty}(\tau)=a_{k}^{\infty} \tau
$$

dans ce cas, $\bar{G}^{\text {Lie }}$ est de dimension 1, engendré par le générateur infinitésimal (analytique) de $f$ : c'est le pseudo-groupe linéaire dans la variable $\tau$.

- Les invariants analytiques de $f$ sont de la forme

$$
\varphi_{k}^{0}(\tau)=a_{k}^{0} \tau \quad \text { et } \quad \varphi_{k}^{\infty}(\tau)=a_{k}^{\infty}\left(\tau^{q}+b_{k}^{\infty}\right)^{1 / q}
$$

dans ce cas, $\bar{G}^{\text {Lie }}$ est de dimension 2 et se redresse sur le pseudogroupe affine dans la variable $\tau^{q}$.

- Les invariants analytiques de $f$ sont de la forme

$$
\varphi_{k}^{0}(\tau)=a_{k}^{0} \tau /\left(1-b_{k}^{0} \tau^{q}\right)^{1 / q} \quad \text { et } \quad \varphi_{k}^{\infty}(\tau)=a_{k}^{\infty} \tau
$$

dans ce cas, $\bar{G}^{\text {Lie }}$ est de dimension 2 et se redresse sur le pseudogroupe affine dans la variable $\frac{1}{\tau^{q}}$.

- Les invariants analytiques de $f$ sont de la forme

$$
\varphi_{k}^{0}(\tau)=a_{k}^{0} \tau /\left(1-b_{k}^{0} \tau^{q}\right)^{1 / q} \quad \text { et } \quad \varphi_{k}^{\infty}(\tau)=a_{k}^{\infty}\left(\tau^{q}+b_{k}^{\infty}\right)^{1 / q}
$$

dans ce cas, $\bar{G}^{\text {Lie }}$ est de dimension 3 et se redresse sur le pseudogroupe de Mobius dans la variable $\tau^{q}$. 
Démonstration. Rappelons d'abord que $f$ admet, sur chaque pétale de Leau $V_{k}^{ \pm}$, un générateur infinitésimal $X_{k}^{ \pm}$: on a $f=\exp \left(X_{k}^{ \pm}\right) \operatorname{sur} V_{k}^{ \pm}$. Par ailleurs, $f$ admet un générateur infinitésimal formel $\hat{X} \in \widehat{\mathcal{X}}(\mathbb{C}, 0)$ qui est $p$-sommable, où $p$ est le contact de $f$ à l'identité. En fait, les $X_{k}^{ \pm}$sont les $p$-sommes sectorielles de $\hat{X}$. De la même manière, les itérées complexes formelles $f^{\circ t}=\exp (t \hat{X}) \in \widehat{\operatorname{Diff}}(\mathbb{C}, 0)$ de $f$ sont $p$-sommables et les $p$ sommes sont données par la collection $\operatorname{des} \exp \left(t X_{k}^{ \pm}\right)$pour $t \in \mathbb{C}$.

En reprenant les arguments de la preuve de la Proposition 4.49, on voit que chaque itérée complexe $f^{\circ t}$ est solution formelle des équations différentielles définissant $\bar{G}^{\text {Lie }}$. Par conséquent, les sommes sectorielles $\exp \left(t X_{k}^{ \pm}\right)$ sont aussi solutions des mêmes équations différentielles et sont des éléments du pseudo-groupe $\bar{G}^{\text {Lie }}$.

Maintenant, il est facile de voir que le pseudo-groupe engendré sur $U^{*}$ par les flots sectoriels $\exp \left(t X_{k}^{ \pm}\right)$est de dimension finie si, et seulement si, on est dans la liste de l'énoncé. On peut voir ça en travaillant dans la coordonnée $\tau$ sur les sphères du chapelet associé à $f$ : sur $S_{k}^{ \pm}$, le flot $\exp \left(t X_{k}^{ \pm}\right)$est le flot linéaire $\tau \mapsto e^{2 i \pi t} \tau$. Par exemple, le flot linéaire sur $S_{k}^{-}$agit au voisinage de 0 dans $S_{k}^{+}$via le recollement $\varphi_{k}^{0}$ : sa dynamique sur $S_{k}^{+}$est engendrée par le flot de $\left(\varphi_{k}^{0}\right)^{*} \tau \partial_{\tau}$. En reprenant les arguments de la preuve du Théorème 2.22 , on montre que le sous-groupe de $\operatorname{Diff}(\mathbb{C}, 0)$ engendré par les deux groupes à 1 paramètre $\exp \left(t \tau \partial_{\tau}\right)$ et $\left(\varphi_{k}^{0}\right)^{*} \exp \left(t \tau \partial_{\tau}\right)$ est résoluble si, et seulement si, $\varphi_{k}^{0}(\tau)=a_{k}^{0} \tau /\left(1-b_{k}^{0} \tau^{q_{k}}\right)^{1 / q}$ pour un $q_{k} \in$ $\mathbb{N}^{*}$. Dans ce cas, le pseudo-groupe engendré est

- $\mathbb{A}_{q_{k}}$ lorsque $b_{k}^{0} \neq 0$ et son algèbre de Lie est $\mathcal{A}_{k}^{0}:=\mathbb{C} \tau \partial_{\tau}+\mathbb{C} \tau^{q_{k}+1} \partial_{\tau}$,

- $\mathbb{L}$ lorsque $b_{k}^{0}=0$ et son algèbre de Lie est $\mathcal{A}_{k}^{0}:=\mathbb{C} \tau \partial_{\tau}+\mathbb{C}$.

Ces algèbres de Lie étant invariantes par le flot linéaire, elles sont en fait définies sur les sphères $S_{k}^{ \pm}$toutes entières comme sous-algèbres de Lie de l'algèbre de Lie $\mathcal{A}$ de $\bar{G}^{\text {Lie }}$ par exemple dans le sens de la section 4.4.3 $\left(\bar{G}^{\text {Lie }}\right.$ est fermé pour la convergence uniforme d'après la Proposition 4.41). Une étude similaire de comparaison de ces algèbres de Lie via les recollements $\varphi_{k}^{\infty}$ montre d'une part que $q_{k}=q$ est le même pour tout $k=0, \ldots, p-1$ puis que les recollements sont de la forme $\varphi_{k}^{\infty}(\tau)=a_{k}^{\infty}\left(\tau^{q}+b_{k}^{\infty}\right)^{1 / q}$. La dimension de l'algèbre de Lie $\mathcal{A}$ dépend de la nullité des $b_{k}^{0}$ et des $b_{k}^{\infty}$.

Si les invariants analytiques $\varphi_{k}^{0}$ et $\varphi_{k}^{\infty}$ ne sont pas de la forme précédente, alors $\mathcal{A}$ est de dimension infinie et $\bar{G}^{L i e}=\operatorname{Diff}(U)$. Si, par contre, on est dans l'une des situations de la liste de l'énoncé, alors $\mathcal{A}$ est de dimension $\leq 3$ et les flots sectoriels $\exp \left(t X_{k}^{ \pm}\right)$engendrent bien un pseudo-groupe de dimension finie sur $U^{*}$. Il reste à vérifier que c'est un pseudo-groupe de Lie en 0 . Pour cela, on considère la coordonnée $\psi$ donnée par $\log (\tau), \tau^{q}$ ou $\frac{1}{\tau^{q}}$ selon le cas et il suffit de vérifier que $\alpha(z)=\psi^{\prime}(z), \beta(z)=\frac{\alpha^{\prime}(z)}{\alpha(z)}$ ou $\gamma(z)=\beta^{\prime}(z)-\frac{1}{2} \beta^{2}(z)$ est à croissance polynomiale en 0 selon la dimension recherchée. Par exemple, pour $\psi(z)=\tau^{q}, \gamma(z)$ est bien définie sur $U^{*}$ 
(invariante par les recollements $\varphi_{k}^{0}$ et $\varphi_{k}^{\infty}$ dans chacun des cas); si elle est à croissance modérée en 0 , alors elle est se prolonge méromorphiquement en 0 par Riemann et le pseudo-groupe satisfait l'équation méromorphe (4.5) $: \bar{G}^{\text {Lie }}$ est de dimension $\leq 3$. Nous laissons ces détails au lecteur. 



\section{Deuxième partie}

\section{Singularités de feuilletages holomorphes en dimension 2}




\section{Chapitre 5}

\section{Feuilletages holomorphes et pseudo-groupes}

Toutes les notions que nous allons rappeler ici sont classiques, au moins dans le cas régulier. Le lecteur pourra trouver plus de détails par exemple dans [85].

\subsection{Feuilletages réguliers sur les surfaces com- plexes}

Un feuilletage holomorphe régulier $\mathcal{F}$ sur une surface complexe $M$ est défini par un atlas dont les cartes

$$
\left(H_{i}: U_{i} \rightarrow \mathbb{C}\right)_{i \in I}
$$

sont des submersions holomorphes satisfaisant, pour chaque intersection $U_{i} \cap U_{j}$ non vide, la condition de recollement

$$
H_{j}=\varphi_{j, i} \circ H_{i}
$$

pour un difféomorphisme holomorphe $\varphi_{j, i}: H_{i}\left(U_{i} \cap U_{j}\right) \rightarrow H_{j}\left(U_{i} \cap U_{j}\right)$.

Puisque les fonctions $H_{i}: U_{i} \rightarrow \mathbb{C}$ sont des submersions, les courbes de niveau $\left\{H_{i}(p)=\right.$ constante $\}$ sont des sous-variétés complexes de dimension 1. Les composantes connexes de ces courbes, appelées plaques, sont les classes d'équivalence pour une relation $\mathcal{F}_{i}$ sur $U_{i}$. Précisément, deux points $p, q \in U_{i}$ sont équivalents si et seulement s'ils appartiennent à la même plaque, i.e. ils peuvent être joints par un chemin $\gamma:[0,1] \rightarrow U_{i}, \gamma(0)=$ $p$ et $\gamma(1)=q$, satisfaisant $H_{i} \circ \gamma(t) \equiv H_{i}(p)$. Le feuilletage global $\mathcal{F}$ est la relation d'équivalence engendrée sur $M$ par les feuilletages locaux $\mathcal{F}_{i}$; les feuilles du feuilletage $\mathcal{F}$ sont les classes d'équivalence pour cette 
relation. Sur chaque intersection $U_{i} \cap U_{j}$, la relation d'équivalence induite par $\mathcal{F}_{i}$ et $\mathcal{F}_{j}$ coïncident grâce aux conditions de recollement : les plaques de $U_{i}$ et $U_{j}$ se recollent deux à deux pour former des surfaces de Riemann plongées dans $U_{i} \cup U_{j}$. La feuille passant par un point $p_{0} \in U_{i}$ s'obtient, partant de la plaque initiale $\left\{H_{i}(p)=H_{i}\left(p_{0}\right)\right\}$, en recollant de proche en proche toutes les plaques possibles à l'aide des conditions de recollement. Chaque feuille est une surface de Riemann connexe immergée dans $M$; son intersection avec chaque carte $U_{i}$ est une réunion dénombrable de plaques et est maximale (pour l'inclusion) à satisfaire à ces propriétés. Les feuilles ne sont pas proprement plongées en général : leur intersection avec une carte donnée $U_{i}$ peut être la réunion d'une infinité de plaques, et même être dense dans $U_{i}$. La restriction d'un feuilletage $\mathcal{F}$ à un ouvert $U \subset M$ a un sens évident avec nos définitions, mais notons que les feuilles de $\left.\mathcal{F}\right|_{U}$ ne sont pas les restrictions des feuilles de $\mathcal{F}$ à $U$, mais leurs composentes connexes par arcs. Un autre atlas de submersions $\left(\widetilde{H}_{i}: \widetilde{U}_{i} \rightarrow \mathbb{C}\right)_{i \in \widetilde{I}}$ comme audessus va définir le même feuilletage $\mathcal{F}$ sur $M$ s'il définit la même relation d'équivalence, i.e. les mêmes feuilles.

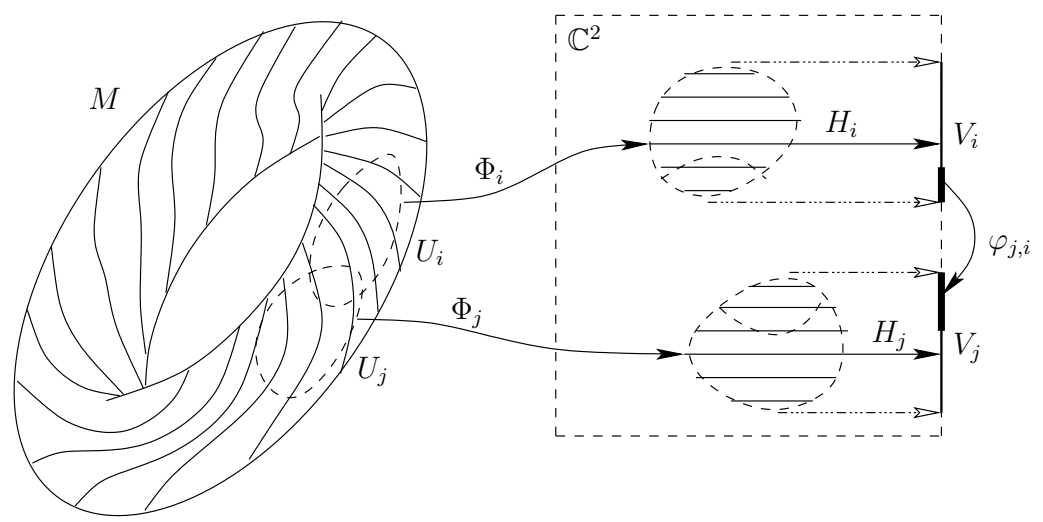

FIGURE 5.1 - Feuilletage

On peut aussi définir un feuilletage $\mathcal{F}$ sur $M$ par un sous-fibré en droites $\mathcal{L} \subset T M$ du fibré tangent. Les feuilles sont alors les surfaces de Riemann connexes immergées maximales dont l'espace tangent appartient en tout point au sous-fibré $\mathcal{L}$. En effet, un tel sous-fibré est défini par un atlas de cartes $\left(U_{i}\right)_{i \in I}$ équipées de champs de vecteurs holomorphes réguliers $\left(X_{i}\right)_{i \in I}$ satisfaisant, sur chaque intersection $U_{i} \cap U_{j}$, les conditions de recollement $X_{j}=u_{j, i} X_{i}$ pour des fonctions holomorphes non nulles $u_{j, i}:\left(U_{i} \cap U_{j}\right) \rightarrow \mathbb{C}^{*}$. Quitte à raffiner l'atlas, il existe pour chaque carte $U_{i}$ un difféomorphisme holomorphe $\Phi_{i}: U_{i} \rightarrow V_{i} \subset \mathbb{C}^{2}$ conjugant le champ de vecteurs $X_{i}$ avec le champ constant $\left(\Phi_{i}\right)_{*} X_{i}=\partial_{x}$. On définit alors 
les plaques sur $U_{i}$ comme étant les courbes intégrales de $X_{i}$. Puisque les courbes intégrales de $\partial_{x}$ sont aussi les courbes de niveau de la submersion $(x, y) \mapsto y$, les plaques sur $U_{i}$ sont aussi les courbes de niveau de la submersion $H_{i}:=\varphi_{i}$ donnée par la seconde coordonnée de $\Phi_{i}=\left(\phi_{i}, \varphi_{i}\right)$. Réciproquement, un feuilletage $\mathcal{F}$ sur $M$ définit un sous-fibré en droites du fibré tangent $T_{\mathcal{F}} \subset T M$ juste en associant, pour tout point $p \in M$, l'espace tangent de la plaque passant par $p$. Les plaques sont alors les courbes intégrales des sections locales $X_{i}$ de ce sous-fibré.

Finalement, on peut encore définir un feuilletage $\mathcal{F}$ sur une surface $M$, ou plutôt le fibré sous-jacent $T_{\mathcal{F}} \subset T M$, par un sous-fibré $N_{\mathcal{F}}^{*} \subset T M^{*}$ du dual : les sections de $N_{\mathcal{F}}^{*}$ sont les sections de $T M^{*}$ qui s'annulent identiquement sur $T_{\mathcal{F}}$. Un tel sous-fibré est défini par un atlas de cartes $\left(U_{i}\right)_{i \in I}$ équipées de 1-formes holomorphes régulières $\left(\omega_{i}\right)_{i \in I}$ satisfaisant, sur chaque intersection $U_{i} \cap U_{j}$, aux conditions de recollement $\omega_{j}=v_{j, i} \omega_{i}$ pour des fonctions holomorphes non nulles $v_{j, i}:\left(U_{i} \cap U_{j}\right) \rightarrow \mathbb{C}^{*}$. Dans des cooronnées locales $\left(x_{i}, y_{i}\right) \in U_{i}$, si $X_{i}=f_{i}\left(x_{i}, y_{i}\right) \partial_{x_{i}}+g_{i}\left(x_{i}, y_{i}\right) \partial_{y_{i}}$ est une section régulière de $T_{\mathcal{F}}$, alors on peut choisir $\omega_{i}=g_{i}\left(x_{i}, y_{i}\right) d x_{i}-f_{i}\left(x_{i}, y_{i}\right) d y_{i}$ pour engendrer $N_{\mathcal{F}}^{*}$. Alors les plaques sont les graphes des solutions $y_{i}\left(x_{i}\right)$ de l'équation différentielle $\frac{d y_{i}}{d x_{i}}=\frac{g_{i}\left(x_{i}, y_{i}\right)}{f_{i}\left(x_{i}, y_{i}\right)}$ dans ces coordonnées. Les feuilles sont alors les surfaces de Riemann connexes immergées maximales dans $M$ en restriction auxquelles les $\omega_{i}$ s'annulent identiquement.

\subsection{Pseudo-groupes d'un feuilletage}

Un feuilletage $\mathcal{F}$ sur une variété $M$ définit 3 pseudo-groupes. Nous renvoyons à la section 4.4 pour la définition de pseudo-groupe qui reste la même en toute dimension.

Tout d'abord, le pseudo-groupe basique de $\mathcal{F}$ est la collection des difféomorphismes holomorphes $\varphi: U \rightarrow V$ entre ouverts de $M$ envoyant les feuilles locales dans les feuilles : si $\left.\mathcal{F}\right|_{U}$ désigne le feuilletage restreint à $U$, alors on demande que chaque feuille de $\left.\mathcal{F}\right|_{U}$ soit envoyée par $\varphi$ dans une feuille de $\mathcal{F}$. Dans des coordonnées locales $(x, y) \in U$ où le feuilletage est défini par $\{y=$ constant $\}$, la restriction du pseudo-groupe basique consiste en tous les difféomorphismes dans $U$ de la forme $\Phi(x, y)=(\phi(x, y), \varphi(y))$. Malgrange l'appelle "pseudo-groupe des automorphismes du feuilletage" dans [127], et le note $\operatorname{Aut}(\mathcal{F})$.

Le pseudo-groupe tangent $G$ de $\mathcal{F}$ est contenu dans le précédent et un peu plus subtil à définir. Considérons d'abord, dans des coordonnées locales $(x, y) \in U$ où le feuilletage est défini par $\{y=$ constant $\}$, le pseudogroupe $G_{U}$ qui consiste en tous les difféomorphismes dans $U$ de la forme $\Phi(x, y)=(\phi(x, y), y)$. Alors le pseudo-groupe tangent $G$ est engendré sur $M$ par tous les $G_{U_{i}}$ où $\left(U_{i}\right)_{i \in I}$ est un atlas de cartes définissant le feuilletage sur $M$. On peut voir aussi $G$ comme le pseudo-groupe engendré sur $M$ par 
les flots des sections holomorphes du fibré tangent $T_{\mathcal{F}}$. C'est le pseudogroupe du transport holonome.

Il est clair que le pseudo-groupe tangent $G$ est contenu dans le pseudogroupe basique. Mais notons que la restriction $\left.G\right|_{U}$ de $G$ à une carte locale $U$ comme au-dessus est en général plus grande que $G_{U}$ : certains éléments de $G$ permutent non trivialement les plaques, i.e. sont de la forme $\Phi(x, y)=(\phi(x, y), \varphi(y))$ avec $\varphi \neq$ identité. En effet, l'action de $G$ est clairement transitive sur chaque feuille $L$ (parce que c'est déjà le cas localement); donc, $\left.G\right|_{U}$ agit transitivement sur la restriction $\left.L\right|_{U}$ alors que $G_{U}$ n'agit transitivement que sur les composantes connexes de $\left.L\right|_{U}$. Néanmoins, l'intersection de chaque feuille avec $U$ ayant une quantité au plus dénombrable de composantes connexes, on voit facilement qu'il n'y a qu'une quantité dénombrable de permutations $\varphi$ possibles apparaissant dans $\left.G\right|_{U}$ (au moins pour une raison d'analyticité). Ainsi, ce pseudo-groupe contient localement des informations globales non triviales sur le feuilletage et le comportement des feuilles.

Les deux pseudo-groupes précédents caractérisent le feuilletage et sont toujours de dimension infinie, ne serait-ce que parce qu'ils le sont déjà dans les cartes $U_{i}$. Nous voudrions maintenant oublier le feuilletage pour nous concentrer sur la partie transverse du pseudo-groupe tangent, à savoir les permutations $\varphi$ des plaques qui apparaissent dans les cartes. Ceci nous conduit à définir le pseudo-groupe transverse de $\mathcal{F}$. Pour cela, considérons un atlas de submersions $\left(H_{i}: U_{i} \rightarrow V_{i} \subset \mathbb{C}\right)_{i \in I}$ définissant le feuilletage $\mathcal{F}$ sur $M$ et considérons l'union disjointe $T=\sqcup_{i \in I} V_{i}$. Rappelons que l'on peut toujours supposer $I$ dénombrable de sorte que $T$ soit une variété (abstraite) de dimension 1. Alors, $T$ est naturellement équipé du pseudogroupe $G$ engendré par les applications de transition $\varphi_{j, i}: H_{i}\left(U_{i} \cap U_{j}\right) \rightarrow$ $H_{j}\left(U_{i} \cap U_{j}\right)$. Si $(x, y) \in U$ sont des coordonnées locales dans lesquelles une des submersions $H: U \rightarrow V$ de la collection précédente s'écrit $H(x, y)=y$ (le feuilletage est alors décrit par $\{y=$ constante $\}$ ), alors la restriction de $G$ à $V$ est précisément la collection des transformations $\varphi$ apparaissant dans les éléments $\Phi(x, y)=(\phi(x, y), \varphi(y))$ du pseudo-groupe tangent. En ce sens, $G$ est la partie transverse du pseudo-groupe tangent.

Il est naturel de dire que deux pseudo-groupes $G$ et $G^{\prime}$ sur des variétés $M$ et $M^{\prime}$ sont équivalents si, et seulement si, il existe un difféomorphisme holomorphe $\Phi: M \rightarrow M^{\prime}$ conjugant $G$ à $G^{\prime}$. Par exemple, si $\Phi$ conjugue deux feuilletages $\mathcal{F}$ et $\mathcal{F}^{\prime}$, alors $\Phi$ conjugue les pseudo-groupes basiques et tangents correspondant. Néanmoins, cette relation d'équivalence est trop fine pour les pseudo-groupes transverses. En effet, le pseudo-groupe transverse construit ci-dessus dépend fortement de l'atlas de submersions choisi pour définir le feuilletage; plus précisément, la variété $T$ dépend du choix des cartes. Nous allons donc définir une relation d'équivalence plus grossière sur les pseudo-groupes (transverses) de sorte que ces objets deviennent intrinsèquement définis par le feuilletage. 
La relation d'équivalence est engendrée par la règle suivante. Soit $G$ un pseudo-groupe sur une variété $M$. Pour tout recouvrement ouvert $M=$ $\cup_{i \in I} U_{i}$, observons que le pseudo-groupe $G$ est encore engendré sur $M$ par toutes les collections $\mathcal{C}_{i, j} \subset G$ d'éléments $\varphi: U \rightarrow V$ satisfaisant $U \subset U_{i}$ et $V \subset U_{j}, i, j \in I$. On peut s'en convaincre en considérant le groupoïde de germes associé. Considérons maintenant la nouvelle variété définie par l'union disjointe $M^{\prime}:=\sqcup_{i \in I} U_{i}$ et équipons-la du pseudo-groupe $G^{\prime}$ engendré par l'ensemble des collections $\mathcal{C}_{i, j} \subset G$. En particulier, $\mathcal{C}_{i, j}$ contient l'application $i d: U_{i} \cap U_{j} \rightarrow U_{i} \cap U_{j}$ qui, dans $M^{\prime}$, devient non triviale et garde mémoire du fait que les $U_{i}$ étaient collés aux $U_{j}$ dans le passé. On dira que les pseudo-groupes $(M, G)$ et $\left(M^{\prime}, G^{\prime}\right)$ sont équivalents.

Grosso-modo, cette règle signifie que nous nous autorisons à disconnecter $M$ en plusieurs (éventuellement une infinité dénombrable) de composantes en ajoutant au pseudo-groupe les difféomorphismes qui permettent de recoller les morceaux pour revenir sur $M$. En considérant la relation d'équivalence engendrée par cette règle, nous nous autorisons réciproquement à remplacer $M$ par la variété $M^{\prime}$ obtenue par chirurgies dénombrables, en recollant des ouverts de $M$ à l'aide d'éléments de $G$. Attention, bien que $G$ soit dénombrable, on ne peut pas pour autant tout identifier. En effet, si l'on identifie tous les points de $M$ appartenant à la même orbite sous l'action de $G$, alors le quotient topologique $M / G$ est encore défini par un atlas de cartes $U_{i} \rightarrow U_{i} / G$, mais les applications de transition $\varphi_{i, j}$ ne satisfont pas en général la condition de cocycle

$$
\varphi_{i, j} \circ \varphi_{j, k} \circ \varphi_{k, i}=\text { identité. }
$$

Si $(T, G)$ est le pseudo-groupe transverse d'un feuilletage $\mathcal{F}$ sur $M$, le quotient $T / G$ est l'espace des feuilles du feuilletage. Si une feuille de $\mathcal{F}$ est dense dans $M$, alors le point de $T / G$ correspondant est dense dans $T / G$ par rapport à la topologie quotient. Le pseudo-groupe $(T, G)$ peut-être considéré comme un atlas définissant $T / G$ sur lequel des structures géométriques supplémentaires peuvent apparaître. Avec la définition d'équivalence que nous venons de décrire, un pseudo-groupe $\left(T^{\prime}, G^{\prime}\right)$ équivalent à $(T, G)$ est un autre atlas définissant le même quotient $T / G$ avec la même structure analytique. De cette manière, on se convainc que le pseudogroupe transverse de $\mathcal{F}$ est précisément défini modulo l'équivalence cidessus.

Si $T$ supporte une structure géométrique additionnelle, comme par exemple une métrique Riemannienne, qui soit invariante sous l'action de $G$, alors on dira que c'est une structure géométrique transverse à $\mathcal{F}$ et par exemple, dans ce cas précis, que $\mathcal{F}$ est transversalement Riemannien. Alors $T / G$ est naturellement équipé de cette structure géométrique qui du coup se relève sur $T^{\prime}$, invariante sous l'action de $G^{\prime}$, pour tout pseudo-groupe $\left(T^{\prime}, G^{\prime}\right)$ équivalent à $(T, G)$. 
On dira qu'un pseudo-groupe $(T, G)$ est trivial s'il est équivalent au pseudo-groupe trivial sur une variété, c'est-à-dire si le quotient $T / G$ est elle même une variété.

Exemple 5.1. Les pseudo-groupes $(\mathbb{C}, G)$ des translations sur $\mathbb{C}$ et $\left(\mathbb{C}^{*}, G^{\prime}\right)$ des homothéties sur $\mathbb{C}^{*}$ sont équivalents. En effet, on peut découper le premier en union disjointes $M=\sqcup_{n \in \mathbb{Z}} B_{n}$ bandes horizontales $B_{n}=$ $\{n \pi<\operatorname{Im}(z)<(n+2) \pi\}$ et le pseudo-groupe $G$ induit sur $\mathbb{C}$ est engendré par la collection des difféomorphismes entre ouverts de $B_{n}$ et $B_{m}$ de la forme $z \mapsto z+t, t \in \mathbb{C}$. Le second peut-être découpé en union disjointe $M=\sqcup_{n \in \mathbb{Z}} S_{n}$ de secteurs $S_{n}=\{n \pi<\arg (z)<(n+2) \pi\}$ (une infinité de copies de $S_{0}$ et $S_{1}$ ) et le pseudo-groupe $G^{\prime}$ induit sur $M^{\prime}$ est engendré par tous les difféomorphismes entre ouverts de $S_{n}$ et $S_{m}$ de la forme $z \mapsto c z, c \in \mathbb{C}^{*}$. Maintenant, la collection des difféomorphismes $\Phi_{n}: B_{n} \rightarrow S_{n} ; z \mapsto e^{2 i \pi z}$ définit un difféomorphisme global $\Phi: M \rightarrow M^{\prime}$ conjugant les pseudo-groupes respectifs $G$ et $G^{\prime}$. Notons que "ce pseudogroupe" n'est pas trivial. Attention, le pseudo-groupe défini par les homothéties sur $\mathbb{C}$ tout entier n'est pas équivalent au précédent.

Donnons maintenant quelques indications permettant de calculer le pseudo-groupe transverse d'un feuilletage dans bien des cas, c'est-à-dire de décrire un représentant $(T, G)$ de la relation d'équivalence à l'aide de ses générateurs sur $T$.

Considérons un feuilletage $\mathcal{F}$ sur $M$. Par une courbe $T$ transverse $\grave{a} \mathcal{F}$ on entendra une variété $T$ de dimension 1 (non nécessairement connexe) munie d'une immersion injective $i: T \hookrightarrow M$ transverse à $\mathcal{F}$. On dira que $T$ est une transversale complète si toutes les feuilles intersectent $T$ (i.e. son image). Le pseudo-groupe tangent de $\mathcal{F}$ contient des difféomorphismes $\Phi: U \rightarrow V$ entre ouverts de $M$ préservant la transversale, i.e. envoyant $T \cap U$ dans $T$; un tel élément $\Phi$ induit un difféomorphisme $\varphi=\left.\Phi\right|_{T \cap U}:(T \cap U) \rightarrow(T \cap V)$ entre ouverts de $T$. La collection de toutes ces applications $\varphi$ est un pseudogroupe sur $T$, appelé le pseudo-groupe induit par $\mathcal{F}$ sur la transversale $T$.

Considérons un atlas dénombrable de submersions $\left(H_{i}: U_{i} \rightarrow V_{i} \subset\right.$ $\mathbb{C})_{i \in I}$ définissant le feuilletage $\mathcal{F}$ sur $M$. Pour chaque $i \in I$, choisissons une section holomorphe $s_{i}: V_{i} \hookrightarrow U_{i}$ de $H_{i}$ : la courbe $T_{i}:=s_{i}\left(V_{i}\right) \subset$ $U_{i}$ est automatiquement transverse au feuilletage et, quitte à raffiner le recouvrement $\left(U_{i}\right)_{i \in I}$, on peut supposer de plus que ces courbes $T_{i}$ sont deux à deux disjointes. Par construction, la courbe $T=\cup_{i \in I} T_{i}$ est une transversale complète à $\mathcal{F}$ et le pseudo-groupe induit par $\mathcal{F}$ sur $T$ est le pseudo-groupe transverse du feuilletage.

Soit $G$ un pseudo-groupe sur une variété $T$. Si un ouvert $T^{\prime} \subset T$ intersecte toutes les orbites pour l'action de $G$ sur $T$, alors le pseudo-groupe restreint $\left(T^{\prime}, G^{\prime}:=\left.G\right|_{U}\right)$ est équivalent à $(T, G)$. En effet, pour chaque point $p \in T \backslash T^{\prime}$, il existe un élément $\varphi_{p}: U_{p} \rightarrow V_{p} \subset T^{\prime}$ dans $G$; il est possible d'extraire, du recouvrement $\cup_{p \in T \backslash T^{\prime}} U_{p}$ de $p \in T \backslash T^{\prime}$, un recouvrement 
dénombrable $\cup_{i \in I} U_{p_{i}}$. Maintenant, découpons d'abord $T$ en $T^{\prime} \sqcup\left(\sqcup_{i \in I} U_{p_{i}}\right)$, puis recollons les ouverts $U_{p_{i}}$ avec $T^{\prime}$ à l'aide des $\varphi_{p_{i}}: U_{p_{i}} \rightarrow V_{p_{i}} \subset T^{\prime}$.

Par conséquent, le pseudo-groupe induit par le feuilletage $\mathcal{F}$ sur une transversale complète $T$ dans $M$ est le pseudo-groupe transverse du feuilletage.

\subsection{Holonomie d'une feuille, d'un feuilletage}

Le pseudo-groupe induit par $\mathcal{F}$ sur une courbe transverse $T$ est engendré par les applications d'holonomie définies comme suit. Pour tout chemin $\gamma:[0,1] \rightarrow M$ tangent au feuilletage (i.e. contenu dans une feuille de $\mathcal{F}$ ) avec extrémités $\gamma(0)=p_{0}$ et $\gamma(1)=p_{1}$ dans $T$, on peut construire une famille continue d'éléments $\Phi_{t}: U_{0} \rightarrow U_{t} \subset M$ du pseudo-groupe tangent satisfaisant :

- $U_{0}$ contient $p_{0}$ et $\Phi_{0}$ est l'identité sur $U_{0}$;

- $\Phi_{t}\left(p_{0}\right)=\gamma(t)$ pour tout $t \in[0,1]$;

- $\Phi_{1}\left(T \cap U_{0}\right)=T \cap U_{1}$.

On peut faire cela en considérant un recouvremant fini de trivialisations locales pour le feuilletage le long de $\gamma([0,1])$. Alors, l'application d'holonomie $\varphi_{\gamma}:\left(T, p_{0}\right) \rightarrow\left(T, p_{1}\right)$ est le germe de difféomorphisme induit par $\left.\Phi_{1}\right|_{T}$ en $p_{0}$. L'application d'holonomie $\varphi_{\gamma}$ ne dépend ni du choix de la famille $\Phi_{t}$, ni du choix de la classe d'homotopie de $\gamma$ (à extrémités fixes) dans la feuille.

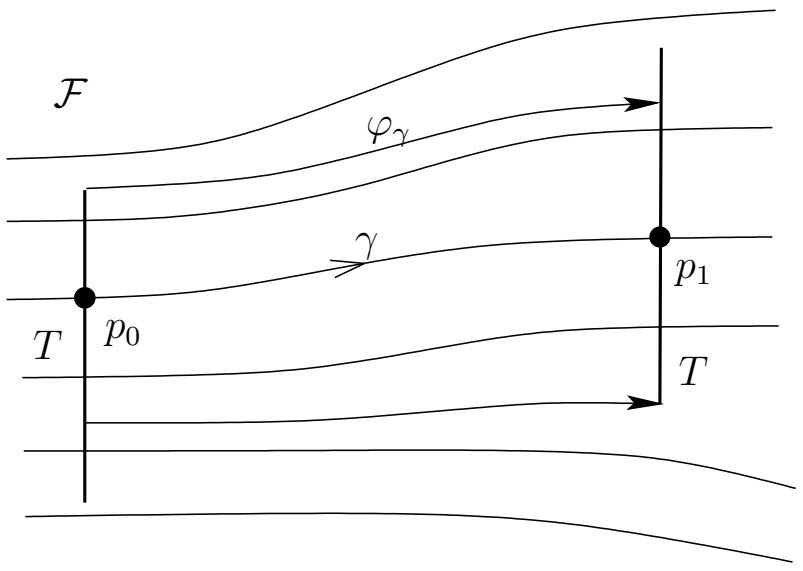

Figure 5.2 - Holonomie d'un chemin entre deux transversales

Voici une autre manière de construire $\varphi_{\gamma}:\left(T, p_{0}\right) \rightarrow\left(T, p_{1}\right)$. Étant donné un atlas de submersions $\left(H_{i}: U_{i} \rightarrow V_{i} \subset \mathbb{C}\right)_{i \in I}$ définissant le feuilletage $\mathcal{F}$ sur $M$, on peut extraire un recouvrement fini de $\gamma([0,1])$ par une 
suite de cartes, disons $U_{0}, U_{1}, \ldots, U_{N}$ pour simplifier, intersectées successivement par $\gamma(t)$ quand $t$ va de 0 à 1 . Par construction, la composition des applications de transition successives $\varphi_{N, N-1} \circ \cdots \varphi_{2,1} \circ \varphi_{1,0}$ est bien définie, quitte à restreindre leur domaine de définition, sur un voisinage ouvert de $H_{0}\left(p_{0}\right)$. Cette application définit un germe de difféomorphisme $\left(V_{0}, H_{0}\left(p_{0}\right)\right) \rightarrow\left(V_{N}, H_{N}\left(p_{1}\right)\right)$ qui correspond à $\varphi_{\gamma}$ à travers les coordonnées locales respectivement induites sur $T$ par $H_{0}$ en $p_{0}$ et par $H_{N}$ en $p_{1}$.

Pour tout point $p_{0} \in T$, on hérite d'une représentation

$$
\pi_{1}\left(\mathcal{F}_{p_{0}}, p_{0}\right) \rightarrow \operatorname{Diff}\left(T, p_{0}\right) ; \gamma \mapsto \varphi_{\gamma}^{\circ(-1)},
$$

du groupe fondamental de la feuille $\mathcal{F}_{p_{0}}$ passant par $p_{0}$ dans le groupe des germes de difféomorphismes de $T$ fixant $p_{0}$. Attention, la flêche naïve $\gamma \mapsto \varphi_{\gamma}$ définit une anti-représentation, i.e. $\varphi_{\gamma_{1} \gamma_{2}}=\varphi_{\gamma_{2}} \circ \varphi_{\gamma_{1}}$. $\mathrm{A}$ travers n'importe quelle coordonnée locale $(\mathbb{C}, 0) \rightarrow\left(T, p_{0}\right)$, on déduit la représentation d'holonomie de la feuille $\mathcal{F}_{p_{0}}$ passant par $p_{0}$

$$
\pi_{1}\left(\mathcal{F}_{p_{0}}, p_{0}\right) \rightarrow \operatorname{Diff}(\mathbb{C}, 0)
$$

qui est bien définie au choix près de la coordonnée, c'est-à-dire à conjugaison près dans $\operatorname{Diff}(\mathbb{C}, 0)$.

\section{$5.4 \quad$ Feuilletages singuliers}

Soit $\mathcal{F}$ un feuilletage régulier défini sur un voisinage épointé $U^{*}=U \backslash\{0\}$ de l'origine $0 \in \mathbb{C}^{2}: \mathcal{F}$ est par exemple défini à partir d'un recouvrement de $U^{*}$ par des cartes $\left(U_{i}\right)_{i \in I}$ équipées de 1 -formes $\omega_{i}=f_{i}(x, y) d x-g_{i}(x, y) d y$. Quitte à changer de coordonnées sur $U$, on peut supposer que le feuilletage $\mathcal{F}$ n'est pas le feuilletage vertical et donc qu'aucune des fonctions holomorphes $g_{i}$ n'est identiquement nulle. Les conditions de recollement entrainent alors que toutes les fonctions méromorphes $\frac{f_{i}(x, y)}{g_{i}(x, y)}$ coïncident et se recollent en une fonction méromorphe globale sur $U^{*}$. Par le Théorème de Levi (Hartogs version méromorphe), cette fonction s'étend méromorphiquement sur $U$, i.e. est donnée au voisinage de 0 par le quotient $\frac{f(x, y)}{g(x, y)}$ de deux fonctions holomorphes $f$ et $g$ en 0 . Par conséquent, le feuilletage régulier $\mathcal{F}$ sur $U^{*}$ est encore défini, près de 0 , par une 1-forme holomorphe $\omega=f(x, y) d x-g(x, y) d y$ éventuellement singulière en 0 . Notons que le feuilletage $\mathcal{F}$ est aussi défini sur le voisinage épointé de 0 par le champ de vecteurs holomorphe à singularité isolée $X=g(x, y) \partial_{x}+f(x, y) \partial_{y}$. Par contre, le feuilletage n'est en général pas défini par une submersion $H: U \rightarrow \mathbb{C}$ holomorphe, ni même méromorphe.

Un feuilletage holomorphe singulier $\mathcal{F}$ sur une surface complexe $M$ est, par définition, un feuilletage régulier $\mathcal{F}$ défini en dehors d'un ensemble 
discret de points de $M$, i.e. un feuilletage à singularités isolées. D'après la discussion précédente, de manière équivalente, un tel objet est défini par un atlas de cartes $\left(U_{i}\right)_{i \in I}$ équipées de 1-formes holomorphes $\left(\omega_{i}\right)_{i \in I}$ (resp. de champs de vecteurs holomorphes $\left.\left(X_{i}\right)_{i \in I}\right)$ à zéros isolés satisfaisant, sur chaque intersection $U_{i} \cap U_{j}$, les conditions de recollement $\omega_{j}=v_{j, i} \omega_{i}$ (resp. $\left.X_{j}=u_{j, i} X_{i}\right)$ pour des fonctions holomorphes $u_{j, i}, v_{j, i}:\left(U_{i} \cap U_{j}\right) \rightarrow \mathbb{C}^{*}$ (automatiquement non nulles). Les singularités du feuilletage $\mathcal{F}$ sont les points en lesquels $\mathcal{F}$ ne peut pas être prolongé en un feuilletage régulier; il est facile de voir que ce sont les zéros (isolés) des formes $\omega_{i}$ ou des champs $X_{i}$ définissant $\mathcal{F}$. On notera $\operatorname{Sing}(\mathcal{F})$ l'ensemble des points singuliers de $\mathcal{F}$. Les feuilles d'un feuilletage singulier sont par définition les feuilles du feuilletage régulier induit sur $M \backslash \operatorname{Sing}(\mathcal{F})$. De même, le pseudo-groupe basique, tangent ou transverse de $\mathcal{F}$ sera par définition celui du feuilletage régulier sous-jacent sur $M \backslash \operatorname{Sing}(\mathcal{F})$.

Remarque 5.2. Une forme différentielle $\omega=f(x, y) d x-g(x, y) d y$ (non identiquement nulle) ayant un zéro non isolé à l'origine définit tout de même un feuilletage régulier $\mathcal{F}$ en dehors de son lieu d'annulation. En divisant $\omega$ par une équation convenable locale de la composante de codimension 1 de ses zéros (i.e. le diviseur commun de $f$ et $g$ ), on obtient une 1-forme $\tilde{\omega}$ holomorphe à singularités isolées dont le feuilletage $\widetilde{\mathcal{F}}$ prolonge celui de $\omega$ en dehors d'un ensemble discret. Le prolongement ainsi obtenu est maximal et souvent appelé feuilletage singulier saturé associé à $\omega$; nous le noterons $\mathcal{F}_{\omega}$. De la même manière, une 1-forme méromorphe (non identiquement nulle) définit un unique feuilletage singulier saturé.

Les sous-fibrés en droites $T_{\mathcal{F}}$ et $N_{\mathcal{F}}^{*}$ définis sur $M \backslash \operatorname{Sing}(\mathcal{F})$ ne s'étendent pas en sous-fibré en droites aux points singuliers. Par contre, en passant aux faisceaux des sections locales, ils définissent des sous- $\mathcal{O}$-module localement libre de rang 1 qui, quant-à-eux, s'étendent aux points singuliers. Réciproquement, tout sous- $\mathcal{O}$-module localement libre de rang 1 du fibré tangent $T M$ ou de son dual $T M^{*}$ définit un feuilletage holomorphe singulier sur $M$. En effet, un tel sous-faisceau est toujours localement engendré par une section à zéros isolés.

Exemple 5.3. Une fonction holomorphe $H:\left(\mathbb{C}^{2}, 0\right) \rightarrow(\mathbb{C}, 0)$ non constante définit un feuilletage singulier $\mathcal{F}$ sur un voisinage $U$ de 0 dont toutes les feuilles sont des sous-variétés analytiques sur $U^{*}$. Si $H$ a une singularité isolée en 0 (dH s'annule) alors la courbe analytique singulière $C=\{H=0\}$ est la réunion disjointe du point singulier $\{0\}$ et d'un nombre fini de feuilles de $\mathcal{F}$ dans $U^{*}$.

Exemple 5.4. Une fonction méromorphe $H:\left(\mathbb{C}^{2}, 0\right) \rightarrow \overline{\mathbb{C}}$ non constante définit un feuilletage singulier $\mathcal{F}$ sur un voisinage $U$ de 0 dont toutes les feuilles sont des sous-variétés analytiques sur $U^{*}$. Si 0 est un point d'indétermination de $H$, alors chaque feuille (dans $U^{*}$ ) se prolonge en une 
courbe analytique singulière en 0 : ce sont les composantes irréductibles des niveaux $\{H=$ constante $\}$.

Exemple 5.5. La 1-forme $\omega=x^{2} d y+y d x$ définit un feuilletage $\mathcal{F}$ au voisinage de 0 dont les feuilles sont:

- la feuille verticale $\{x=0\}$,

- les graphes de $y=c e^{1 / x}, c \in \mathbb{C}$.

Outre la feuille horizontale $\{y=0\}$ donnée par $c=0$, toutes les autres feuilles sont transcendantes dans tout voisinage de 0 et accumulent la feuille verticale : c'est le Théorème de Picard.

On appellera courbe invariante (ou séparatrice) passant par un point $p \in \operatorname{Sing}(\mathcal{F})$ tout germe de courbe analytique $C$ (singulière) en $p$ qui, dans un voisinage épointé de $p$ coïncide avec une réunion (finie) de feuilles de $\mathcal{F}$. Si une courbe $C$ est définie près de $p$ par l'équation $F=0$ avec $F:(M, p) \rightarrow(\mathbb{C}, 0)$ holomorphe réduite, et si $\mathcal{F}$ est défini par un germe de 1-forme holomorphe $\omega$ à singularité isolée en $p$, alors la courbe $C$ est invariante si, et seulement si, $F$ divise $d F \wedge \omega$. Le Théorème de CamachoSad que nous discuterons plus loin affirme que par tout point singulier de $\mathcal{F}$ passe une courbe invariante. On dit que la singularité est dicritique lorsqu'elle admet une infinité de courbes invariantes irréductibles deux-àdeux distinctes; c'est le cas par exemple pour le feuilletage défini par les courbes de niveau d'une fonction méromorphe $H$ au voisinage d'un point d'indétermination. Pour une singularité non dicritique, on parlera de la courbe invariante pour désigner la réunion (finie) de toutes ses courbes invariantes irréductibles.

Une caractérisation remarquable des courbes invariantes est la suivante. Soit $U$ un voisinage d'un point singulier $p$ du feuilletage $\mathcal{F}$ et soit $L$ une feuille du feuilletage régulier $\left.\mathcal{F}\right|_{U^{*}}$ en restriction au voisinage épointé $U^{*}=$ $U \backslash\{p\}$. Si $L$ est analytique (i.e. fermée) dans $U^{*}$ et adhère à $p$ dans $U$, alors son adhérence $\bar{L}=L \cup\{p\}$ dans $U$ est analytique et définit donc une courbe invariante. On a aussi le :

Lemme 5.6 (Moussu). Si deux germes de feuilletages singuliers $\mathcal{F}$ et $\mathcal{F}^{\prime}$ en $\left(\mathbb{C}^{2}, 0\right)$ possèdent une feuille commune $L$ sur un voisinage épointé $U^{*}$ de 0 , alors

- ou bien $L$ se prolonge en une courbe analytique en 0 ,

- ou bien $\mathcal{F}=\mathcal{F}^{\prime}$.

Démonstration. Soient $\omega$ et $\omega^{\prime}$ deux 1-formes holomorphes à singularité isolée sur $U$ définissant $\mathcal{F}$ et $\mathcal{F}^{\prime}$. Par hypothèse, ces deux formes s'annulent identiquement en restriction à $L$ et donc $\omega \wedge \omega^{\prime}$ s'annule le long de $L$. Dans des coordonnées locales $(x, y)$ en 0 , la 2-forme précédente s'écrit $\omega \wedge \omega^{\prime}=F(x, y) d x \wedge d y$ où $F$ est une fonction holomorphe sur $U$. Si $L$ n'est pas analytique, alors $F$ est identiquement nulle sur $U$ puisqu'elle s'annule le long de $L: \omega \wedge \omega^{\prime} \equiv 0$ et $\mathcal{F}=\mathcal{F}^{\prime}$. 

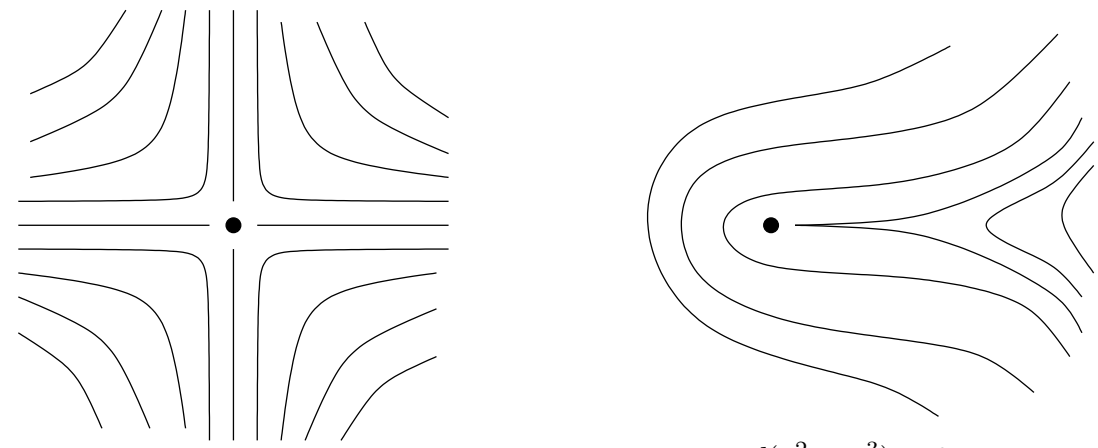

$$
d(x y)=x d y+y d x=0
$$

$$
d\left(y^{2}-x^{3}\right)=0
$$

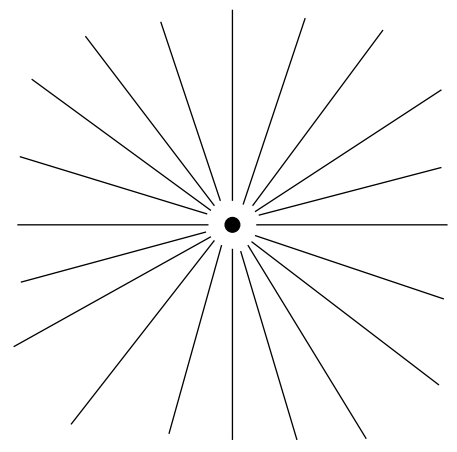

$$
x^{2} d(y / x)=x d y-y d x=0
$$
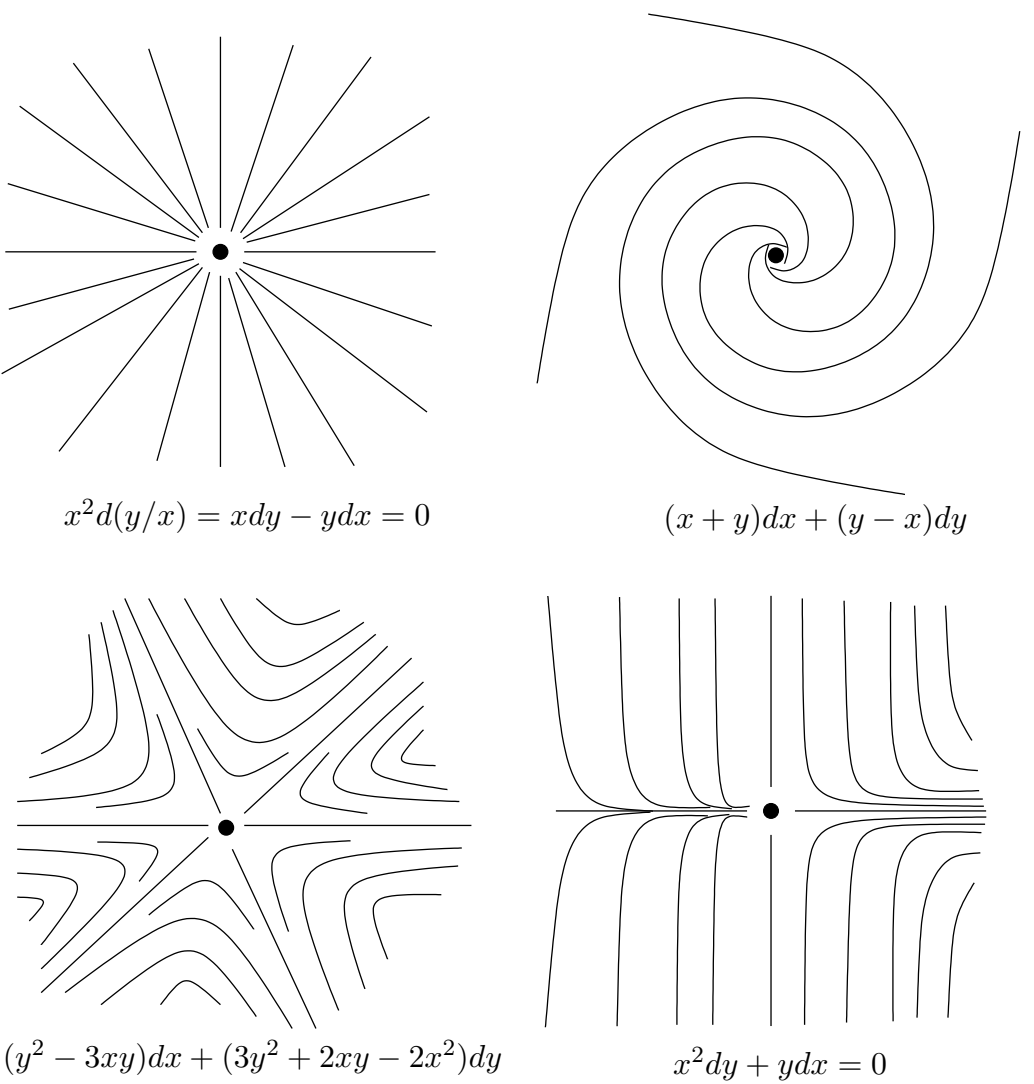

$\left(y^{2}-3 x y\right) d x+\left(3 y^{2}+2 x y-2 x^{2}\right) d y$

Figure 5.3 - Exemples de feuilletages singuliers 


\section{Chapitre 6}

\section{Singularités réduites}

Dans ce chapitre, nous allons décrire les singularités "réduites" de feuilletages holomorphes en dimension 2. Elles apparaîtront plus loin comme singularités terminales après réduction d'une singularité générale par éclatements ponctuels. Ce sont les singularités définies (à changement linéaire près de coordonnées) par :

$$
\omega=x d y-\alpha y d x+\cdots
$$

où $\alpha \in \mathbb{C} \backslash \mathbb{Q}_{>0}$.

\subsection{Bestaire préliminaire des singularités non dégénérées}

Soit $\mathcal{F}$ le germe de feuilletage singulier de $\left(\mathbb{C}^{2}, 0\right)$ défini par un champ de vecteurs holomorphe à singularité isolée

$$
X=f(x, y) \partial_{x}+g(x, y) \partial_{y}
$$

et considérons sa partie linéaire sous forme matricielle :

$$
\operatorname{Lin}(X)=\left(\begin{array}{ll}
a & b \\
c & d
\end{array}\right) \quad \text { où } \quad X=(a x+b y) \partial_{x}+(c x+d y) \partial_{y}+\cdots
$$

Si $\Phi \in \operatorname{Diff}\left(\mathbb{C}^{2}, 0\right)$ a pour partie linéaire

$$
\operatorname{Jac}(\Phi)=\left.\left(\begin{array}{ll}
\frac{\partial \Phi_{1}}{\partial x} & \frac{\partial \Phi_{1}}{\partial y} \\
\frac{\partial \Phi_{2}}{\partial x} & \frac{\partial \Phi_{2}}{\partial y}
\end{array}\right)\right|_{(x, y)=(0,0)}
$$

alors on a :

$$
\operatorname{Lin}\left(\Phi^{*} X\right)=\operatorname{Jac}(\Phi)^{-1} \cdot \operatorname{Lin}(X) \cdot \operatorname{Jac}(\Phi)
$$


Ainsi, les valeurs propres $\left\{\alpha_{1}, \alpha_{2}\right\}$ de $\operatorname{Lin}(X)$ sont des invariants de conjugaison du champ de vecteurs $X$. Un autre champ de vecteurs $X^{\prime}$ (à singularité isolée) va définir le même feuilletage si, et seulement si, $X^{\prime}=F(x, y) X$ pour une fonction non nulle $F$ et on a $\operatorname{Lin}(F X)=F(0,0) \operatorname{Lin}(X)$. Ainsi, à changement de coordonnée près, le feuilletage $\mathcal{F}$ est défini par un champ de vecteurs dont la partie linéaire est de la forme:

$$
\operatorname{Lin}(X)=\left(\begin{array}{ll}
1 & 0 \\
0 & \alpha
\end{array}\right), \quad\left(\begin{array}{ll}
1 & 1 \\
0 & 1
\end{array}\right), \quad\left(\begin{array}{ll}
0 & 1 \\
0 & 0
\end{array}\right) \quad \text { ou } \quad\left(\begin{array}{ll}
0 & 0 \\
0 & 0
\end{array}\right) .
$$

On dira que $\mathcal{F}$ est une singularité dégénérée dans le dernier cas, c'est-àdire lorsque la partie linéaire de $X$ est triviale; c'est le cas de l'exemple en bas à gauche dans la figure 5.3. Ce sont les singularités "de type général" en ce sens où on ne peut espérer de classification raisonnable dans leur ensemble. On verra dans les sections suivantes comment dégager de nombreux invariants de conjugaison, puis comment décrire leur pseudogroupe transverse. On notera deux sous-familles relativement simples qui ont été beaucoup étudiées. La première (voir [50, 227, 228, 155, 130, 158]) est définie par les champs de vecteurs

$$
X=X_{n}+\cdots
$$

s'annulant à l'ordre $n>1$ dont la partie homogène d'ordre $n$ est suffisamment générique : nous verrons dans la section 7.1.1 qu'elles se réduisent après 1 éclatement et que le diviseur exceptionnel est invariant par le feuilletage éclaté; cette propriété les caractérise. La seconde famille est celle des singularités de feuilletages dicritiques $\mathcal{F}$ qui deviennent régulier après un éclatement (voir [57, 99, 156, 79, 157]). Nous reviendrons sur ces familles à titre d'exemple dans les sections qui suivent.

Dans le second cas, lorsque la matrice $\operatorname{Lin}(X)$ est nilpotente (non triviale), c'est-à-dire lorsque $\mathcal{F}$ est défini à changement linéaire de coordonnées près par

$$
X=y \partial_{x}+\cdots \quad \text { ou encore } \omega=y d y+\cdots,
$$

on dit que $\mathcal{F}$ est une singularité nilpotente ; c'est le cas de l'exemple en haut à droite dans la figure 5.3. Ces singularités ont fait l'objet de nombreux travaux (voir $[147,58,74,123,116,144,14,212,211,213,162$, $39,145,81,141]$ ) qui permettent aujourd'hui de bien les comprendre. Leur classification analytique se ramène cependant, dans le cas le plus simple, au problème ouvert discuté dans la section 2.6.

Enfin, quand une des valeurs propres est non nulle, $\mathcal{F}$ est défini dans de bonnes coordonnées par

$$
X=x \partial_{x}+\alpha y \partial_{y}+\cdots \quad \text { ou encore } \quad \omega=x d y-\alpha y d x+\cdots,
$$


(excepté le cas unipotent $\left.X=(x+y) \partial_{x}+y \partial_{y}+\cdots\right)$ avec $\alpha \in \mathbb{C}$. Lorsque $\alpha \neq 0$, on peut permuter les 2 coordonnées : seule la paire $\left\{\alpha, \frac{1}{\alpha}\right\}$ est bien définie par le feuilletage, ou encore, ce qui revient au même, son indice de Baum-Bott $\alpha+\frac{1}{\alpha}+2 \in \mathbb{C}$ (voir [24], page 34). On dira que la singularité est de type :

- hyperbolique si $\alpha \notin \mathbb{R}$,

- nœud si $\alpha>0$ (incluant le cas unipotent),

- $\operatorname{col}$ si $\alpha<0$,

- nœud-col si $\alpha=0$.

Lorsque $\alpha \notin \mathbb{R}_{\leq 0}$, on dit que $\mathcal{F}$ est dans le domaine de Poincaré en référence au théorème de linéarisation démontré par Henri Poincaré pour les germes de champs de vecteurs analytiques (toute dimension). Dans ce cas, on peut linéariser le feuilletage (et même le champ de vecteurs) excepté dans quelques cas résonants pour lesquels Henri Dulac a cependant donné des formes normales très simples :

Théorème 6.1 (Poincaré-Dulac). Soit $\mathcal{F}$ une singularité de feuilletage non dégénérée d'invariant $\alpha \in \mathbb{C} \backslash \mathbb{R}_{\leq 0}$.

1- Si $\alpha \notin \mathbb{N}$ et $\frac{1}{\alpha} \notin \mathbb{N}$ alors le feuilletage est linéarisable : dans de bonnes coordonnées holomorphes, $\mathcal{F}$ est défini par

$$
\omega=x d y-\alpha y d x .
$$

2- Sinon, disons $\alpha=n \in \mathbb{N}^{*}$, alors il existe des coordonnées holomorphes dans lesquelles le feuilletage $\mathcal{F}$ est défini par

$$
\omega=x d y-n y d x \quad \text { ou } \quad x d y-\left(n y+x^{n}\right) d x .
$$

Dans le cas $n=1$ de l'énoncé, on trouve les singularités radiale et unipotente respectivement. On appelle singularité de Poincaré-Dulac celles qui apparaissent à droite dans le second cas : ce sont celles qui ne sont pas diagonalisables.

Lorsque $\alpha \leq 0$, on dit que $\mathcal{F}$ est dans le domaine de Siegel ; dans ce cas, des problèmes de divergence viennent s'ajouter aux problèmes de résonance comme nous le verrons plus loin. Le définition qui suit est motivée par le théorème de Seidenberg (voir section 7.1.3)

Définition 6.2 (Singularité réduite). On dira qu'une singularité de feuilletage $\mathcal{F}$ est une singularité réduite lorsqu'elle est définie par

$$
\omega=x d y-\alpha y d x+\cdots
$$

avec $\alpha \in \mathbb{C} \backslash \mathbb{Q}_{>0}$.

Comme nous le verrons, ce sont précisément les singularités non dégénérées possédant exactement deux courbes invariantes (ou séparatrices) formelles transverses; les nœuds que l'on a exclu en ont une infinité (cas linéaire) ou une seule (cas Poincaré-Dulac). 


\subsection{Singularités réduites linéaires}

Les singularités linéaires $\omega=x d y-\alpha y d x, \alpha \in \mathbb{C} \backslash \mathbb{Q} \geq 0$, possèdent deux courbes invariantes, à savoir les axes de coordonnées. Les autres droites horizontales et verticales sont transverses au feuilletage. L'holonomie de l'axe des $x$ se calcule sur une transversale $\left\{x=x_{0}\right\}$ paramétrée par la variable $y$ et s'écrit $f_{x_{0}}(y)=e^{2 i \pi \alpha} y$. En effet, une intégrale première multiforme est donnée par $H(x, y)=x^{-\alpha} y$, l'holonomie est définie par :

$$
H\left(e^{2 i \pi} x_{0}, f_{x_{0}}(y)\right)=H\left(x_{0}, y\right)
$$

et la monodromie de $H$ est donnée par $H\left(e^{2 i \pi} x, e^{2 i \pi \alpha} y\right)=H(x, y)$.

L'holonomie de l'axe des $y$ se calcule, par exemple, en inversant les rôles de $x$ et $y$, ce qui nous donne $\frac{\omega}{\alpha}=y d x+\frac{1}{\alpha} x d y$ et $g_{y_{0}}(x)=e^{2 i \pi / \alpha} x$.
6.2.1 Type $(\text { Fin })_{\text {col }}: \omega=p y d x+q x d y$ avec $p, q \in \mathbb{N}^{*}(\alpha=$ $\left.-\frac{p}{q} \in \mathbb{Q}<0\right)$

Ce sont les seules singularités réduites admettant une intégrale première holomorphe : $H(x, y)=x^{p} y^{q}$. On parlera alors de cols holomorphes.

\subsubsection{Type $(\operatorname{Lin})_{\text {col }}: \omega=x d y-\alpha y d x$ avec $\alpha \in \mathbb{R}_{<0} \backslash \mathbb{Q}_{<0}$}

Ce sont les cols linéaires; l'holonomie $y \mapsto e^{2 i \pi \alpha} y$ est une rotation irrationnelle; l'adhérence de toute feuille autre que les axes est une sousvariété (lisse) analytique réelle de dimension 3 (elle n'adhère ni aux axes, ni à l'origine).

6.2.3 Type $(\operatorname{Lin})_{\text {nœud }}: \omega=x d y-\alpha y d x$ avec $\alpha \in \mathbb{R}_{>0} \backslash \mathbb{Q}_{>0}$

Ce sont les nœuds linéaires. L'holonomie $y \mapsto e^{2 i \pi \alpha} y$ est une rotation irrationnelle. L'adhérence de toute feuille autre que les axes est une sousvariété analytique réelle de dimension 3 singulière à l'origine; elle sépare tout voisinage de l'origine en 2 composantes connexes, chacune contenant un axe de coordonnée.

\subsubsection{Type $(\operatorname{Lin})_{\text {hyp }}: \omega=x d y-\alpha y d x$ avec $\alpha \in \mathbb{C} \backslash \mathbb{R}$}

Ce sont les singularités linéaires hyperboliques; l'holonomie est contractante ou dilatante; l'adhérence de chaque feuille $L$ autre que les axes est :

$$
\bar{L}=L \cup\{\text { axes }\} .
$$




\subsection{Singularités réduites de type col : $\omega=$ $x d y-\alpha y d x+\cdots$ avec $\alpha \in \mathbb{R}_{<0}$}

Les travaux de Briot et Bouquet montrent qu'il existe encore deux courbes invariantes lisses, tangentes aux axes de coordonnées à l'origine. Par changement de coordonnées holomorphes, on peut supposer que ce sont précisément les axes, de sorte que :

$$
\omega=x(1+F(x, y)) d y-\alpha y(1+G(x, y)) d x
$$

où $F, G \in \mathbb{C}\{x, y\}$ s'annulent toutes deux à l'origine (voir [140], p.518-522).

En particulier, sur un petit voisinage de l'origine, les autres droites horizontales et verticales sont transverses au feuilletage. Un petit calcul d'intégration (voir [140], p.481-482) montre que l'holonomie de l'axe des $x$, calculée, par exemple, sur une de ces transversales dans la coordonnée $y$, est de la forme :

$$
f(y)=e^{2 i \pi \alpha} y+\cdots .
$$

Comme précédemment, en inversant les rôles de $x$ et $y$, on déduit que l'holonomie de l'axe des $y$ est du type $g(x)=e^{2 i \pi / \alpha} x+\cdots$.

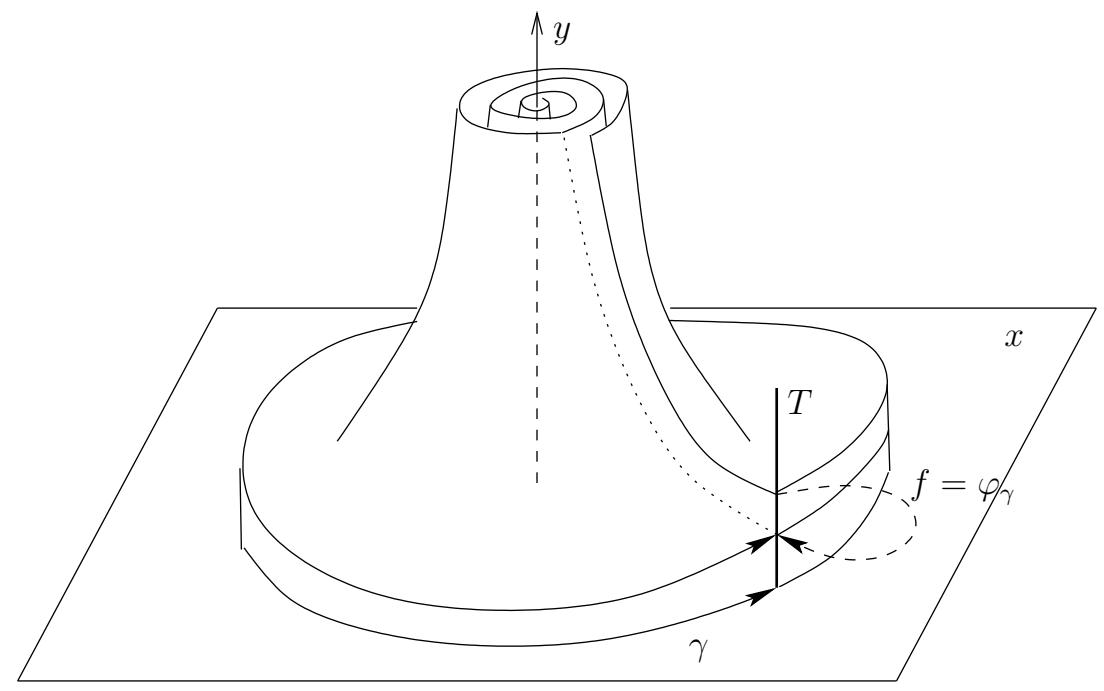

Figure 6.1 - Holonomie d'un col

Théorème 6.3 (Mattei-Moussu). Fixons $\alpha \in \mathbb{R}_{<0}$. Alors le feuilletage défini par $\omega=x d y-\alpha y d x+\cdots$ est caractérisé par l'holonomie de sa séparatrice horizontale : si $\omega^{\prime}$ est aussi de la forme précédente, alors sont équivalents 
- $\mathcal{F}_{\omega}$ et $\mathcal{F}_{\omega}^{\prime}$ sont analytiquement équivalents (par un germe de difféomorphisme ne permutant pas les axes),

- leurs holonomies $f, f^{\prime}=e^{2 i \pi \alpha} y+\cdots$ sont analytiquement conjuguées.

Démonstration. Le sens direct est évident. Pour la réciproque, nous allons construire le changement de coordonnées sous la forme :

$$
\Phi(x, y)=(x, \phi(x, y))
$$

c'est-à-dire respectant la fibration $\{x=C \underline{t e}\}$ fibre par fibre. Avant cela, une homothétie permet de supposer $\omega$ et $\omega^{\prime}$ définies au-delà du polydisque $\mathbb{D} \times \mathbb{D}$ et du type $\frac{d y}{d x}=\alpha \frac{y}{x}(1+\varepsilon(x, y))$ avec $|\varepsilon(x, y)|<\varepsilon<<1$.

Par un premier changement de coordonnée du type $(x, \phi(y))$, on se ramène à :

$$
\frac{d y}{d x}=\alpha \frac{y}{x}(1+x \varepsilon(x, y))
$$

Pour cela, on décompose $\varepsilon=y \varepsilon_{1}(y)+x \varepsilon_{2}(x, y)$; il suffit de choisir $\phi(y)$ satisfaisant $\frac{d \phi}{\phi}=\frac{d y}{y\left(1+y \varepsilon_{1}(y)\right)}$. Cette dernière équation s'intègre en posant $\phi=u y$.

Par hypothèse, il existe un difféomorphisme $\varphi \in \operatorname{Diff}(\mathbb{C}, 0)$ conjugant les holonomies $f(y)$ et $f^{\prime}(y)$ calculées sur la transversale $\{x=1\}$. À homothétie près, on peut supposer $\varphi$ bien défini sur $\mathbb{D}$. Le changement de coordonnée $\Phi$ est alors donné par :

$$
\Phi(x, y)=\left(x, \varphi_{\gamma}^{\prime} \circ \varphi \circ \varphi_{\gamma}^{\circ(-1)}(y)\right)
$$

où $\varphi_{\gamma}$ et $\varphi_{\gamma}^{\prime}$ sont les applications d'holonomie des feuilletages respectifs $\mathcal{F}_{\omega}$ et $\mathcal{F}_{\omega^{\prime}}$ associées à un chemin $\gamma$ joignant le point 1 au point $x$ dans la base $\mathbb{D}^{*} \times\{0\}$.

Cette application est holomorphe, ne dépend pas du choix de $\gamma$ puisque $\varphi_{*} f=f^{\prime}$ et envoie feuilles de $\mathcal{F}_{\omega}$ sur celles de $\mathcal{F}_{\omega^{\prime}}$. La chose à démontrer est que cette application est bien définie et bornée sur un domaine $\mathbb{D}^{*} \times \mathbb{D}_{\varepsilon}$ où $\mathbb{D}_{\varepsilon}$ désigne un petit disque de centre 0 et de rayon $\varepsilon>0$. Pour cela, nous allons montrer que les applications d'holonomie $\varphi_{\gamma}(y)$ sont uniformément proches des applications correspondantes du modèle linéaire dès que l'on borne la longueur de $\gamma$. Ceci entrainera automatiquement cela en choisissant une longueur permettant à $\gamma$ d'atteindre tous les points de la base, et $\Phi$ s'étendra automatiquement par Riemann le long de l'axe vertical.

Pour le modèle linéaire, les applications d'holonomie sont de la forme $\varphi_{\gamma}^{\operatorname{lin}}(y)=\left(\frac{x_{0}}{x}\right)^{\alpha} y$ où $x_{0}$ et $x$ sont les extrémités de $\gamma$, la détermination dépendant de la classe d'homotopie de $\gamma$. Considérons donc la variable $\varphi=\left(\frac{x_{0}}{x}\right)^{\alpha} y$ et estimons sa variation le long des feuilles du feuilletage :

$$
\frac{d \varphi}{\varphi}=-\alpha \frac{d x}{x}+\frac{d y}{y}=\alpha \varepsilon(x, y) d x
$$




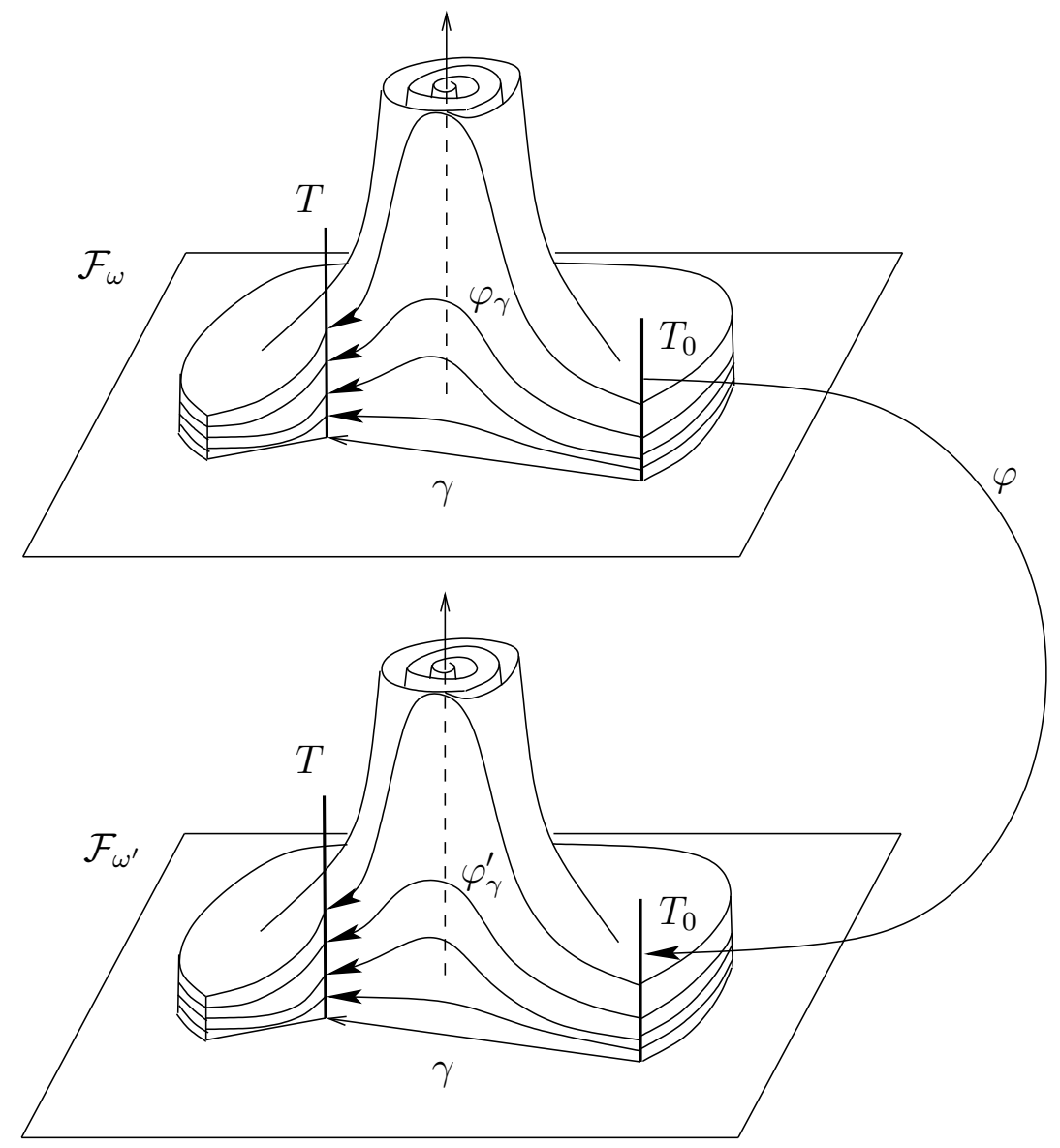

FiguRE 6.2 - Extension aux feuilletages d'une conjugaison entre les holonomies 
de sorte que :

$$
\left|\frac{d \varphi}{\varphi}\right| \leq \varepsilon|\alpha d x|
$$

d'où, en intégrant le long d'un chemin $\gamma$ de longueur $\leq \delta$ :

$$
\left|\log \left(x_{1}^{\alpha} y_{1}\right)-\log \left(x_{0}^{\alpha} y_{0}\right)\right| \leq \varepsilon \delta|\alpha|
$$

ce qui nous donne finalement :

$$
0<e^{-\varepsilon \delta|\alpha|} \leq\left|\frac{\varphi_{\gamma}(y)}{\varphi_{\gamma}^{\operatorname{lin}}(y)}\right| \leq e^{\varepsilon \delta|\alpha|}<\infty
$$

uniformément pour tout chemin $\gamma$ de longueur $\leq \delta$. En particulier, cette estimation vaut aussi pour $\varphi_{\gamma}^{\circ(-1)}=\varphi_{-\gamma}(y)$.

Une autre preuve, techniquement plus élémentaire, mais plus longue, se trouve dans [66].

Remarque 6.4. Si l'on note $y \mapsto f_{x_{0}}(y)$ l'holonomie calculée sur la transversale $\left\{x=x_{0}\right\}$ dans la variable $y$, alors la flêche $(x, y) \mapsto\left(x, f_{x}(y)\right)$ définit un difféomorphisme (fibré laissant le feuilletage invariant) qui se prolonge sur $\{x=0\}$ par $f_{0}(y)=e^{2 i \pi \alpha} y$.

Remarque 6.5. Toute feuille qui adhère à un axe adhère aussi à l'autre.

Remarque 6.6. L'holonomie de l'une ou l'autre des séparatrices est linéarisable si, et seulement si, le col est linéarisable. En particulier, l'holonomie d'une séparatrice est linéarisable (resp. périodique) si, et seulement si, il en va de même pour l'autre.

Remarque 6.7. Tout col est linéarisable par une transformation fibrée $\Phi(x, y)=(x, \phi(x, y))$ au-dessus d'un disque coupé $\left\{x \notin \mathbb{R}_{\geq 0}\right\}$. La monodromie de $\Phi$ est donnée par :

$$
\phi\left(e^{2 i \pi} x, y\right)=\phi\left(x, e^{-2 i \pi \alpha} f_{x}(y)\right) \quad \text { pour } \quad x \in \mathbb{R}_{\geq 0} .
$$

Ainsi, le feuilletage est construit de la façon suivante. On a coupé le col linéaire $\omega=x d y-\alpha y d x$ au-dessus de $\left\{x \in \mathbb{R}_{\geq 0}\right\}$, puis on l'a recollé au-dessus de $\left\{x \in \mathbb{R}_{>0}\right\}$ en décalant les feuilles à l'aide de la partie non linéaire, par exemple, de $f_{1}(y)$ donnée par l'automorphisme tangent à l'identité $h(y):=e^{-2 i \pi \alpha} f_{1}(y)$.

Le résultat suivant est démontré dans [138] dans le cas $\alpha \in \mathbb{Q}<0$, puis dans [171] dans le cas d'un col général.

Théorème 6.8 (Perez-Marco-Yoccoz). Étant donnés $\alpha \in \mathbb{R}_{<0}$ et $f(z)=$ $e^{2 i \pi \alpha} z+\cdots \in \operatorname{Diff}(\mathbb{C}, 0)$, il existe $\omega=x d y-\alpha y d x+\cdots$ dont l'holonomie de la séparatrice horizontale est précisément $f$. 
La démonstration suivante reprend celle de Bernard Malgrange dans [126].

Idée de démonstration. La démarche à suivre est suggérée par la remarque 6.7. On considère l'holonomie à réaliser sous la forme $f(z)=e^{2 i \pi \alpha} h(z)$ avec $h(z)$ tangent à l'identité. On construit, à la manière de la remarque 6.4, une transformation fibrée $\Phi(x, y)=(x, \phi(x, y))$ au-dessus du demi-disque $\Delta:=\left\{|\arg (x)|<\frac{\pi}{2},|x|<1\right\}$ laissant invariant le feuilletage linéaire et coïncidant avec $h(y)$ le long de la transversale $\{x=1\}$ :

$$
\phi(x, y)=\left(\varphi_{\gamma}^{\operatorname{lin}}\right)_{*} h(y)=x^{\alpha} h\left(x^{-\alpha} y\right)
$$

où $\varphi_{\gamma}^{\text {lin }}$ désigne l'application d'holonomie associée au chemin $\gamma=[1, x]$. En décalant les feuilles du feuilletage linéaire par $\Phi$, on construit une variété de dimension 2 complexe $M$ munie d'un feuilletage dont l'holonomie est $f$. Il faut reconnaitre $M$ comme étant un voisinage de l'origine de $\mathbb{C}^{2}$ privé de l'axe vertical. Pour cela, on utilise une construction intermédiaire $C^{\infty}$.

On construit, à l'aide d'une partition de l'unité portant sur l'argument de $x$, une transformation $C^{\infty}$ fibrée $\widetilde{\Phi}$ au-dessus du demi-disque $\Delta$ qui, au-dessus du sous-secteur $\left\{-\frac{\pi}{2}<\arg (x)<-\frac{\pi}{4}\right\}$, est l'identité et, audessus du sous-secteur $\left\{\frac{\pi}{4}<\arg (x)<\frac{\pi}{2}\right\}$, est exactement $\Phi$. En décalant les feuilles du feuilletage linéaire avec $\widetilde{\Phi}$, on récupère un feuilletage $C^{\infty}$ sur $\mathbb{D}^{*} \times \mathbb{D}_{\varepsilon}$ ainsi qu'une structure presque complexe intégrable qui en fait un feuilletage holomorphe. La structure presque complexe se prolonge de manière $C^{\infty}$ le long de l'axe vertical car la transformation $\widetilde{\Phi}$ y est, par construction, infiniment tangente à l'identité. Elle reste bien sûr intégrable lorsqu'on la prolonge. Le théorème de Newlander-Nirenberg nous donne un système de coordonnées $C^{\infty}$ qui redresse la structure presque complexe, et par suite, le feuilletage $C^{\infty}$ sur un feuilletage holomorphe qui se prolonge par Riemann sur la droite verticale.

Ainsi, il existe de nombreux cols non linéarisable que nous allons maintenant décrire.

\subsubsection{Type $(\operatorname{Lin})_{\mathrm{col}}^{\mathrm{ex}}: \omega=x d y-\alpha y d x+\cdots$ non linéari- sable, $\alpha \in \mathbb{R}_{<0} \backslash \mathbb{Q}_{<0}$}

Ce sont les cols à holonomie de type (Lin) ${ }^{\text {ex }}$. Dans ce cas, $\alpha$ n'est pas un nombre de Brjuno, $\alpha \notin \mathcal{B}$, ce qui donne à ces singularités un caractère exceptionnel.

\subsubsection{Type $(\text { Eucl })_{\mathrm{col}}$ et $(\text { Eucl })_{\mathrm{col}}^{\mathrm{ex}}: \omega=p y d x+q x d y+\cdots$ non linéarisable}

Ce sont les cols à holonomie de type (Eucl) ou (Eucl) ${ }^{\text {ex }}$ appelés aussi cols résonants; ils sont étudiés dans [139]. Dulac a montré qu'une telle 
singularité est formellement conjuguée à un des modèles $\omega_{p / q, k, \lambda}=p\left(1+(\lambda-1) u^{k}\right) y d x+q\left(1+\lambda u^{k}\right) x d y, \quad u:=x^{p} y^{q}, k \in \mathbb{N}^{*}, \lambda \in \mathbb{C}$ unique (à permutation près des axes de coordonnées). Pour ce dernier, l'holonomie des deux axes de coordonnées (qui sont tous deux courbes invariantes du modèle) est respectivement donnée par :

$$
f=e^{-2 i \pi p / q} \exp \left(X_{k q, \lambda q / p}\right) \quad \text { et } \quad g=e^{-2 i \pi q / p} \exp \left(X_{k p,(1-\lambda) p / q}\right)
$$

où $X_{p, \lambda}=\frac{z^{p+1}}{1+\frac{\lambda}{2 i \pi} z^{p}} \partial_{z}$ (comme dans la Proposition 2.3) et $z$ est une variable homothétique à $y$ et $x$ respectivement.

En effet, notons d'abord que le feuilletage est aussi défini par la forme fermée :

$$
\tilde{\omega}_{p / q, k, \lambda}:=\frac{\omega_{p / q, k, \lambda}}{x y u^{k}}=\frac{d u}{u^{k+1}}+\lambda \frac{d u}{u}-p \frac{d x}{x}
$$

(où nous reconnaitrons plus loin la forme normale formelle du nœud-col) ce qui nous donne l'intégrale première multiforme

$$
H_{p / q, k, \lambda}=x^{p} u^{-\lambda} e^{1 / k u^{k}} .
$$

Nous commençons par calculer l'itérée $q$-ème $f^{\circ q}$ de l'holonomie. Pour une condition initiale $y_{0}$ proche de 0 , le relevé $\gamma$ du lacet $[0,1] \rightarrow\{y=$ $0\} ; \theta \mapsto\left(e^{2 i \pi q \theta}, 0\right)$ dans le feuilletage reste proche du relevé $\gamma_{0}$ dans le feuilletage défini par $d u=0$; notons que $\gamma_{0}(1)=\gamma_{0}(0)=\left(1, y_{0}\right)$ et $\gamma(1)=$ $\left(1, f^{\circ q}\left(y_{0}\right)\right)$. En particulier, si $\delta$ est un petit chemin joignant $y_{0}$ à $f^{\circ q}\left(y_{0}\right)$ dans la transversale $x=1$, alors le composé $\gamma_{0} \cdot \delta$ est homotope à $\gamma$ dans le complémentaire des pôles $u=0$ de la forme fermée $\tilde{\omega}_{p / q, k, \lambda}$, de sorte que

$$
\int_{\gamma_{0}} \tilde{\omega}_{p / q, k, \lambda}+\int_{\delta} \tilde{\omega}_{p / q, k, \lambda}=\int_{\gamma} \tilde{\omega}_{p / q, k, \lambda}=0 .
$$

Ainsi,

$$
\int_{\delta} \tilde{\omega}_{p / q, k, \lambda}=-\int_{\gamma_{0}} \tilde{\omega}_{p / q, k, \lambda}=\int_{\gamma_{0}} p \frac{d x}{x}=2 i \pi p q .
$$

En restriction à la transversale $x=1$, la forme fermée s'écrit

$$
\left.\tilde{\omega}_{p / q, k, \lambda}\right|_{x=1}=q\left(\frac{d y}{y^{k q+1}}+\lambda \frac{d y}{y}\right)
$$

dont le champ de vecteur dual est $X=\frac{1}{q} \frac{y^{k q+1}}{1+\lambda y^{k q}} \partial_{y}$. On passe donc de $y_{0}=\delta(0)$ à $f^{\circ q}\left(y_{0}\right)=\delta(1)$ en intégrant le champ $X$ pendant le temps $2 i \pi p q$ de sorte que :

$$
f^{\circ q}=\exp \left(2 i \pi p \frac{y^{k q+1}}{1+\lambda y^{k q}} \partial_{y}\right)
$$


Compte-tenu de la partie linéaire de $f$, il vient

$$
f=e^{-2 i \pi p / q} \exp \left(2 i \pi \frac{p}{q} \frac{y^{k q+1}}{1+\lambda y^{k q}} \partial_{y}\right)
$$

ce qui nous donne la forme annoncée dans la variable $z=a y$ où $a^{k q}=2 i \pi \frac{p}{q}$. Échanger les variables $x$ et $y$ nous fait passer de $\omega_{p / q, k, \lambda}$ à $\omega_{q / p, k, 1-\lambda}$, à changement de coordonnée linéaire près, ce qui nous donne l'holonomie de la séparatrice verticale.

Toute singularité $\omega$ de type (Eucl) $)_{\text {col }}^{\text {ex }}$ est formellement équivalente à un des modèles $\omega_{p / q, k, \lambda}$; les holonomies respectives de $\omega$ et $\omega_{p / q, k, \lambda}$ sont alors formellement conjuguées. Plus précisément, l'analogue formel du Théorème 6.3 est vrai (où l'on remplace "analytiquement" par "formellement").

Exercice 6.9. Quels sont les cols qui ne sont pas caractérisés par leur paire d'holonomies $\{f, g\}$ ? On remarquera que le nombre $\alpha$ caractérisant la partie linéaire du col doit être un nombre quadratique très spécial. Qu'en est-il des nœuds ou des singularités hyperboliques?

\subsection{Singularités réduites de type nœud-col : $\omega=y d x+\cdots(\alpha=0)$}

Les portraits de phase réels des exemples classiques de la figure 6.3 illustrent d'ores et déjà la dissymétrie du nœud-col qui a suggéré son nom. Ne nous y trompons pas, la croissance des feuilles à droite tout comme leur décroissance à gauche ne sont pas modérées mais exponentielles.
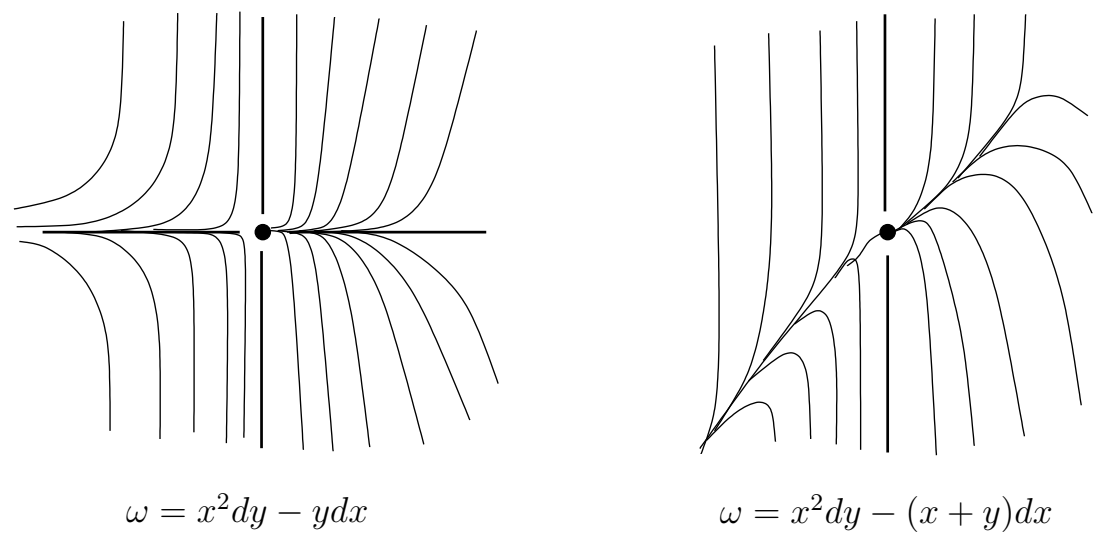

Figure 6.3 - Portrait de phase réel d'un nœud-col 
Les travaux de Briot et Bouquet montrent qu'il y a toujours une courbe invariante lisse tangente à l'axe des $y$ et qu'il existe une autre courbe invariante formelle transverse qui est en général divergente, comme c'est le cas pour l'exemple de droite : elle est paramétrée par la série formelle $y=-\sum_{n>0}(n !) x^{n+1}$, solution de l'équation d'Euler $\omega=0$. La première est la variété forte et la seconde, lorsqu'elle converge, la variété centrale.

Dulac a montré qu'il existait un système de coordonnées dans lequel le nœud-col s'écrit :

$$
\omega=x^{p+1} d y-f(x, y) d x \quad \text { avec } \quad f(0, y)=y \quad \text { et } \quad p \in \mathbb{N}^{*},
$$

de sorte que toutes les droites verticales autres que la variété forte $\{x=0\}$ sont transverses au feuilletage. Ensuite, il montre que $\omega$ se réduit à un unique modèle :

$$
\omega_{p, \lambda}=x^{p+1} d y-y\left(1+\lambda x^{p}\right) d x, \quad \lambda \in \mathbb{C}
$$

par une transformation formelle fibrée $\hat{\phi}(x, y)=(x, \hat{\varphi}(x, y)), \hat{\varphi} \in \mathbb{C}[[x, y]]$.

Par un changement de coordonnée linéaire $z=a x$ où $a^{p}=2 i \pi$, le modèle formel devient :

$$
\widetilde{\omega}_{p, \lambda}=z^{p+1} d y-y\left(2 i \pi+\lambda z^{p}\right) d z ;
$$

ainsi, le champ de vecteurs holomorphe :

$$
\frac{z^{p+1}}{1+\frac{\lambda}{2 i \pi} z^{p}} \partial_{z}+2 i \pi y \partial_{y}
$$

est tangent au feuilletage et son flot au temps 1 nous donne précisément l'holonomie de la variété forte (calculée dans la variable $z$ sur n'importe quelle transversale $\left.\left\{y=y_{0}\right\}\right)$ à savoir $\exp \left(X_{p, \lambda}\right)$. Un petit calcul d'intégration montre que l'holonomie de la variété forte d'un nœud-col général s'écrit (dans la variable $x$ et sur n'importe quelle transversale $y=y_{0}$ ) :

$$
f(x)=x+x^{p+1}+\left(\frac{p+1}{2}-\frac{\lambda}{2 i \pi}\right) x^{2 p+1}+\cdots
$$

de sorte qu'elle est formellement conjuguée à $\exp \left(X_{p, \lambda}\right)$.

On trouve dans [90] la démonstration du

Théorème 6.10 (Hukuhara-Kimura-Matuda). Dans la variable $x$, il existe une collection d'ouverts sectoriels $\left(V_{k}^{-}, V_{k}^{+}\right)_{k \in \mathbb{Z} / p \mathbb{Z}}$ recouvrant un voisinage épointé de $x=0$ du même type que celui de Leau (voir section 3.3 et figure 6.4) et, au-dessus de chacun d'eux, une transformation fibrée :

$$
\phi: V \times \mathbb{D} \hookrightarrow V \times \mathbb{D} ;(x, y)=(x, \varphi(x, y)),
$$

se prolongeant continûment en l'identité le long de la variété forte $\{x=0\}$, et redressant (au-dessus de $V$ ) le noud-col sur le modèle formel correspondant :

$$
\omega \wedge \phi^{*} \omega_{p, \lambda}=0
$$




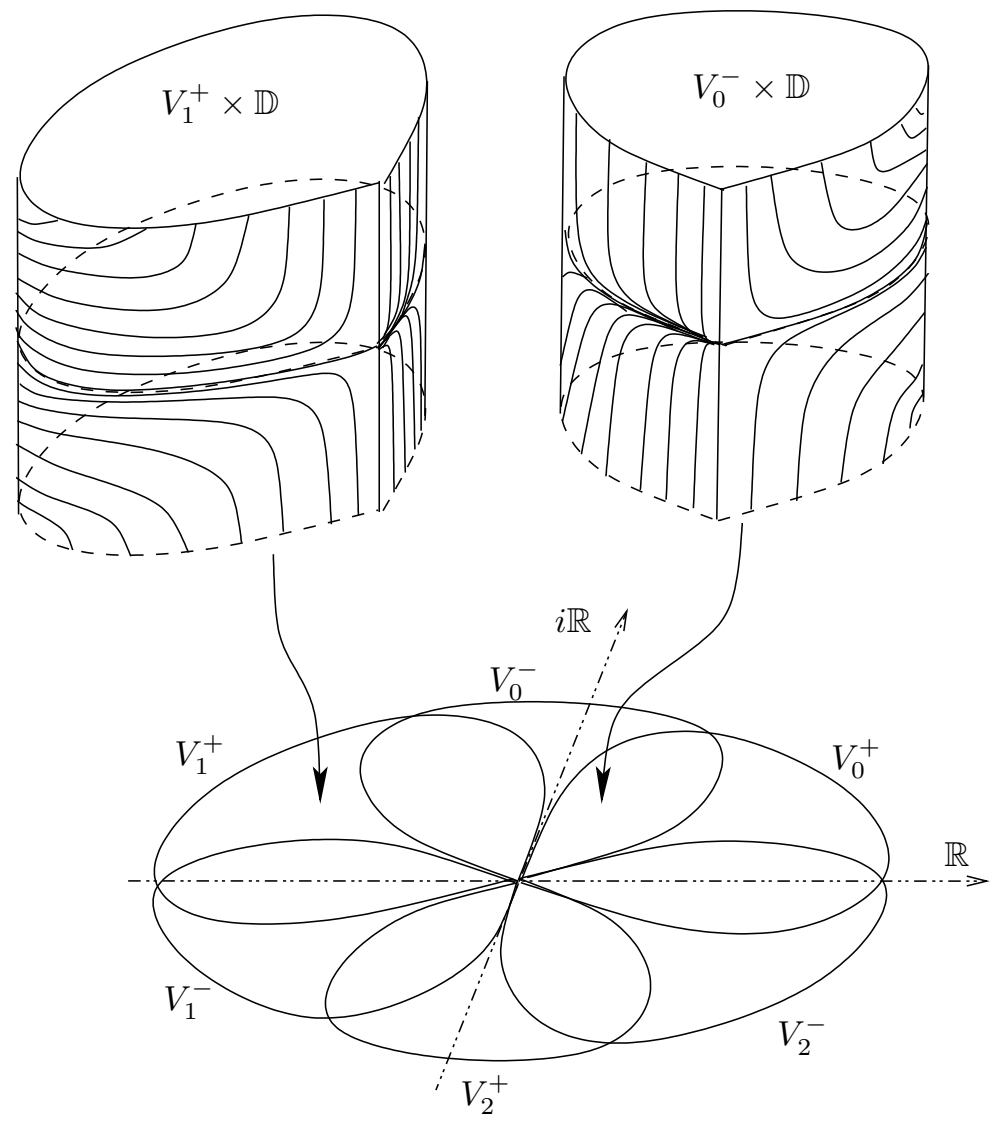

FigURE 6.4 - Normalisation sectorielle du nœud-col 
Le modèle formel $\omega_{p, \lambda}$ admet l'intégrale première multiforme :

$$
H_{p, \lambda}(x, y)=y x^{-\lambda} e^{1 / p x^{p}} .
$$

En raisonnant ou bien directement avec les déterminations sectorielles de cette intégrale première, ou bien, via la transformation sectorielle :

$$
\phi_{p, \lambda}: V \times \mathbb{D} \hookrightarrow V \times \mathbb{C} ;(x, y) \mapsto\left(x /\left(1-\lambda p x^{p} \log (x)\right)^{1 / p}, y\right),
$$

qui envoie $\omega_{p, \lambda}$ sur $\omega_{p, 0}=0$, et l'intégrale première $H_{p, \lambda}$ sur $H_{p, 0}(x, y)=$ $y e^{1 / p x^{p}}$, on vérifie facilement les assertions suivantes.

1- Les feuilles du feuilletage sont simplement connexes au-dessus de chaque pétale $V$ et paramétrées par $\mathbb{C}$.

2- Au-dessus de l'intersection de deux pétales consécutifs $V_{k}^{+} \cap V_{k}^{-}$, les feuilles ont un comportement de type col : elles explosent lorsque l'on s'approche de la singularité. L'intégrale première ne prend ses valeurs que dans un voisinage de $0 \in \mathbb{C}$. L'application de transition $\phi_{k}^{-} \circ\left(\phi_{k}^{+}\right)^{\circ(-1)}$ induit une transformation sectorielle fibrée $\phi_{k}^{0}$ isotrope pour $\omega_{p, \lambda}$, i.e. satisfaisant $\omega_{p, \lambda} \wedge\left(\phi_{k}^{0}\right)^{*} \omega_{p, \lambda}=0$. Elle est décrite, via l'intégrale première $H_{p, \lambda}$, par un germe d'automorphisme $\varphi_{k}^{0} \in \operatorname{Diff}(\mathbb{C}, 0)$.

3- Au-dessus de l'intersection de deux pétales consécutifs $V_{k}^{-} \cap V_{k+1}^{+}$, les feuilles ont un comportement de type noud : elles convergent vers la singularité lorsqu'on s'en approche. L'intégrale première y prend toutes les valeurs de $\mathbb{C}$. L'application de transition $\phi_{k+1}^{+} \circ\left(\phi_{k}^{-}\right)^{\circ}(-1)$ induit une transformation sectorielle fibrée $\phi_{k}^{\infty}$ isotrope pour $\omega_{p, \lambda}$. Elle est décrite, via l'intégrale première $H_{p, \lambda}$, par une transformation affine $\varphi_{k}^{\infty}$.

\subsubsection{L'espace des feuilles}

Il s'obtient en "recollant" les $2 p$ copies de $\mathbb{C}$ correspondant aux $2 p$ paquets de feuilles au-dessus des pétales par les identifications $\varphi_{k}^{0}$ et $\varphi_{k}^{\infty}$ qui rendent compte de la façon dont se recollent les paquets de feuilles sectoriels lorsque l'on tourne autour de la variété forte (voir figure 6.5).

Cet objet est l'espace des feuilles (autres que la variété forte). Lorsque les $\varphi_{k}^{\infty}$ sont tous linéaires, et lorsque le composé

$$
\varphi:=\varphi_{p-1}^{\infty} \circ \varphi_{p-1}^{0} \circ \cdots \circ \varphi_{0}^{\infty} \circ \varphi_{0}^{0}
$$

n'est pas réduit à l'identité, cette construction ne satisfait pas l'axiome de cocycle dans la définition de variété ce qui fait de l'espace des feuilles un objet bien singulier.

\subsubsection{La variété centrale et son holonomie}

La variété centrale existe précisément lorsque les $\varphi_{k}^{\infty}$ sont tous linéaires. En effet, au-dessus de chaque pétale, seule la feuille paramétrée par $0 \in \mathbb{C}$ 


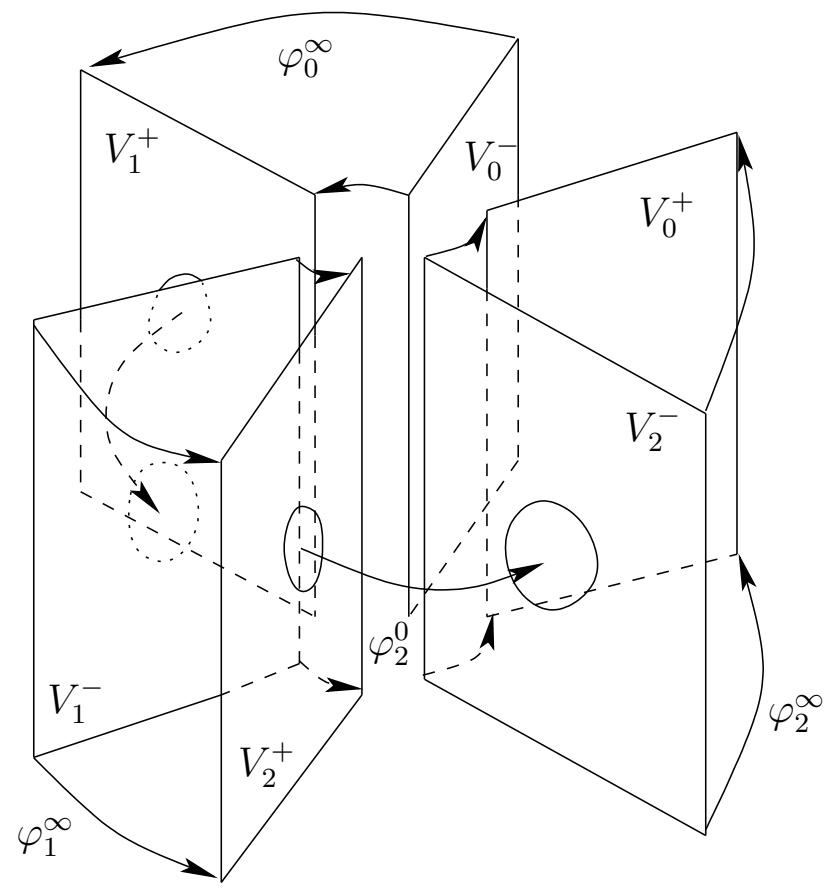

FiguRE 6.5 - Espace des feuilles d'un nœud-col 
a la propriété de converger vers la singularité lorsqu'on s'en approche. Un seul recollement $\varphi_{k}^{\infty}$ non linéaire aurait pour simple conséquence de perdre tout espoir d'avoir une variété centrale puisque la seule feuille candidate à "passer par la singularité" au-dessus de $V_{k}^{-}$se poursuivrait au-dessus de $V_{k+1}^{+}$en "explosant". Par contre, si tous les $\varphi_{k}^{\infty}$ sont linéaires, la feuille paramétrée par $0 \in \mathbb{C}$ tend vers la singularité lorsqu'on s'en approche et donc s'y prolonge analytiquement par Riemann. Ainsi :

la variété centrale formelle du noud-col est convergente si, et seulement si, la partie translation de chaque $\varphi_{k}^{\infty}$ est nulle, i.e. $\varphi_{k}^{\infty}(0)=0$ pour tout $k$.

Lorsque la variété centrale existe, l'holonomie, calculée sur une transversale au-dessus de $V_{0}^{+}$paramétrée par l'intégrale première, est précisément :

$$
\varphi:=\varphi_{p-1}^{\infty} \circ \varphi_{p-1}^{0} \circ \cdots \circ \varphi_{0}^{\infty} \circ \varphi_{0}^{0} .
$$

\subsubsection{L'holonomie de la variété forte}

Rappelons que cette holonomie $f(x)$ est formellement conjuguée à $\exp \left(X_{p, \lambda}\right)$. On voit facilement, en considérant les modèles sectoriels, que toutes les feuilles (exceptée la variété centrale, lorsqu'elle existe) viennent couper la transversale sur laquelle est calculée l'holonomie $f$. On a donc une application holomorphe canonique surjective envoyant le quotient d'un voisinage épointé de $x=0$ par la dynamique de $f$, i.e. le chapelet géométrique associé à $f$, sur l'espace des feuilles. Ceci suggère que les $\varphi_{k}^{0}$ et $\varphi_{k}^{\infty}$ que nous venons de définir ne sont autre que les recollements du chapelet, et l'application est le prolongement analytique des recollements affines $\varphi_{k}^{\infty}$ au plan tout entier. Ceci se vérifie facilement en travaillant sectoriellement avec les modèles formels. On trouve dans [138] le :

Théorème 6.11 (Martinet-Ramis). Étant donné $p \in \mathbb{N}^{*}$, deux nœud-cols du type $x^{p+1} d y-f(x, y) d x=0, f(0, y)=y$, sont analytiquement conjugués si, et seulement si, les holonomies respectives de leur variété forte le sont, c'est-à-dire si et seulement si les applications de recollement des espaces des feuilles $\left(\varphi_{k}^{0}, \varphi_{k}^{\infty}\right)_{k}$ respectifs se correspondent par un changement $d u$ système de coordonnées linéaires.

Toute collection de recollements :

$$
\left(\varphi_{k}^{0}, \varphi_{k}^{\infty}\right)_{k} \in(\operatorname{Diff}(\mathbb{C}, 0) \times \operatorname{Aut}(\mathbb{C}))^{p}
$$

est réalisée par un nœud-col du type $x^{p+1} d y-f(x, y) d x=0$.

Remarque 6.12. Un automorphisme tangent à l'identité à l'ordre $p \in \mathbb{N}^{*}$, $f(z)=z+z^{p+1}+\cdots \in \operatorname{Diff}(\mathbb{C}, 0)$, est l'holonomie de la variété forte d'un nœud-col $x^{p+1} d y-f(x, y) d x=0$ si, et seulement si, tous ses $\varphi_{k}^{\infty}$ sont affines; son inverse est aussi l'holonomie d'un nœud-col si, de plus, tous ses $\varphi_{k}^{0}$ sont homographiques. 
Remarque 6.13. Tout automorphisme $\varphi \in \operatorname{Diff}(\mathbb{C}, 0)$ peut être réalisé comme l'holonomie de la variété centrale d'un nœud-col. Cette holonomie est loin de caractériser la classe analytique de la singularité pour deux raisons. D'abord, la donnée de $\varphi$ ne permet pas de retrouver les recollements intermédiaires $\varphi_{k}^{0}$ qui le composent. Mais même lorsque $p=1$, l'holonomie ne rend compte que de la classe de $\varphi$ modulo changements de coordonnée conforme alors que la classe analytique de la singularité est donnée par la classe de $\varphi$ modulo changements de coordonnée linéaire.

Remarque 6.14. Deux nœuds-cols peuvent être formellement équivalents sans pour autant que les holonomies de leur variété centrale (si elles existent) le soient. En effet, on peut choisir $\varphi$ tangent à l'identité, auquel cas le modèle formel n'a pas d'holonomie. 


\section{Chapitre 7}

\section{Réduction des singularités}

En dimension 2, toute singularité de feuilletage $\mathcal{F}$ se réduit après un nombre fini d'éclatements : le feuilletage relevé $\widetilde{\mathcal{F}}$ n'a plus, le long du diviseur exceptionnel $D$, que des singularités réduites, à savoir celles que nous venons de décrire. Chaque composante irréductible $D_{i}$ de $D$ est ou bien transverse au feuilletage, ou bien invariante. Nous verrons plus loin que le pseudo-groupe transverse à $\mathcal{F}$ ou à $\widetilde{\mathcal{F}}$ est en grande partie engendré par l'holonomie des composantes invariantes. Nous donnons ici tous les ingrédients qui permettront de décrire ce pseudo-groupe dans la suite. Enfin, nous expliquons comment construire une singularité à réduction et à holonomies prescrites, ce qui permettra de réaliser de nombreux pseudogroupes.

\section{$7.1 \quad$ Éclatements}

On appelle éclatement d'un point $p$ d'une surface complexe $M$ la nouvelle surface complexe $\widetilde{M}$ obtenue en remplaçant, dans $M$, le point $p$ par l'ensemble $\mathbb{P}^{1} \simeq \overline{\mathbb{C}}$ des directions passant par ce point, munie de sa projection canonique sur $M$. Détaillons cette construction dans le cas où $M=\mathbb{C}^{2}$ et $p$ est l'origine. La variété éclatée $\widetilde{M}$ est définie par 2 cartes :

$$
(x, t): V_{x} \rightarrow \mathbb{C}^{2} \quad \text { et } \quad(T, y): V_{y} \rightarrow \mathbb{C}^{2}
$$

et par le changement de carte :

$$
\Phi: V_{x} \backslash\{t=0\} \rightarrow V_{y} \backslash\{T=0\} ;(x, t) \mapsto\left(\frac{1}{t}, t x\right) .
$$

La projection canonique $\Pi: \widetilde{M} \rightarrow M=\mathbb{C}^{2}$ est définie sur ces cartes par :

$$
V_{x} \rightarrow \mathbb{C}^{2} ;(x, t) \mapsto(x, t x) \quad \text { et } \quad V_{y} \rightarrow \mathbb{C}^{2} ;(T, y) \mapsto(T y, y) .
$$


La variété $\widetilde{M}$ ainsi construite est la réunion disjointe du diviseur exceptionnel :

$D:=D_{x} \cup D_{y}=\Pi^{-1}(0)$ avec $D_{x}=\{x=0\} \subset V_{x}$ et $D_{y}=\{y=0\} \subset V_{y}$

(isomorphe à $\overline{\mathbb{C}}$ ) et du complémentaire $\widetilde{M} \backslash D$ qui est isomorphe à $\mathbb{C}^{2} \backslash$ $\{(0,0)\}$ par $\Pi$. La surface $M$ est réglée par les droites $\{t=$ constante $\}$ qui se projettent par $\Pi$ sur les droites $\left\{\frac{y}{x}=\right.$ constante $\}$ passant par l'origine.

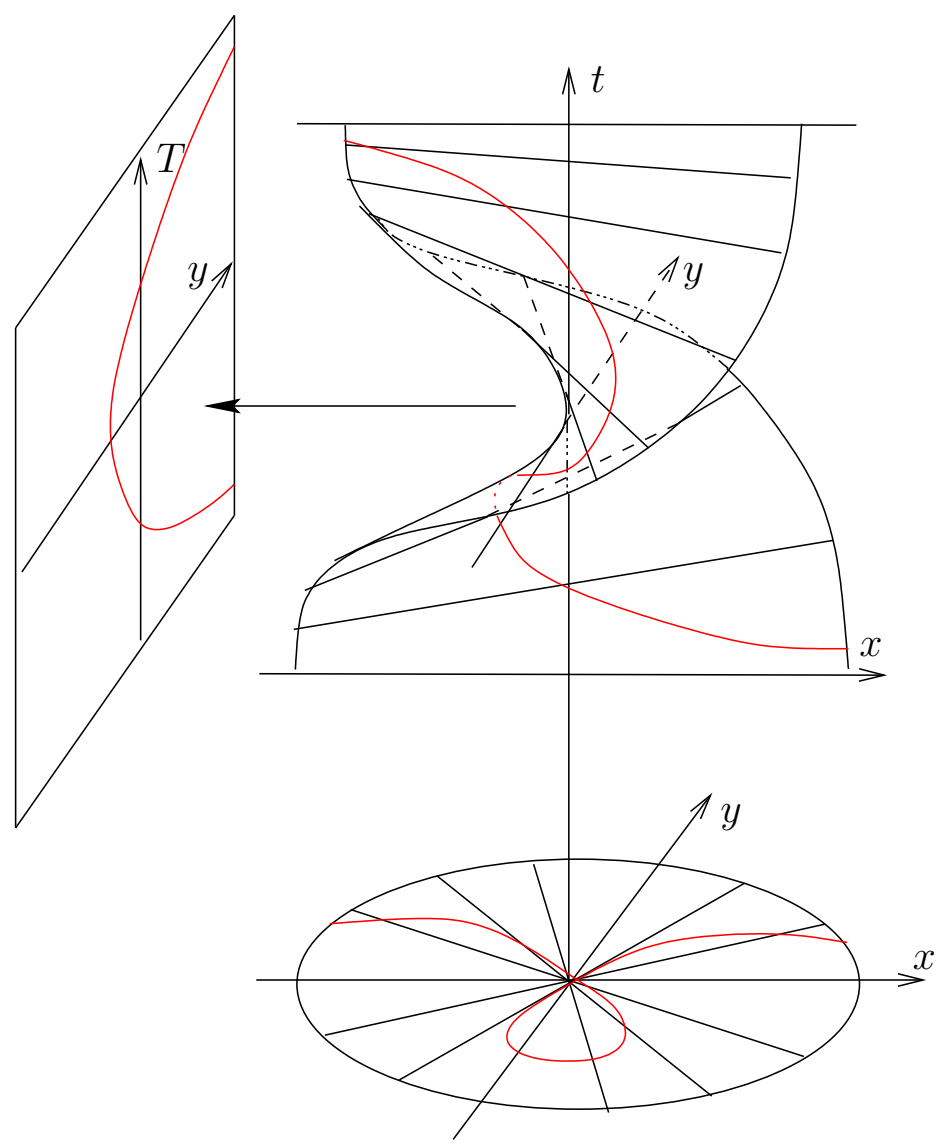

Figure 7.1 - Éclatement de l'origine de $\mathbb{C}^{2}$

L'éclaté $\widetilde{M}$ d'une surface complexe $M$ en un point $p$ se déduit en effectuant la même construction à partir d'un système de coordonnées locales $(x, y)$ de $M$ en $p$. Sur le dessin précédent, la courbe $C$ d'équation $F(x, y)=x^{2}-y^{2}(y+1)$, qui est singulière à l'origine, se relève en une courbe 
lisse $\widetilde{C}$ qui, dans la carte $V_{y}$, a pour équation $\widetilde{F}(x, y)=T^{2}-y-1$. En fait, la fonction $F$ se relève dans la carte $V_{y}$ par $F \circ \Pi(T, y)=y^{2}\left(T^{2}-y-1\right)$; cette fonction s'annule le long du diviseur et, dans cette carte, nous donne l'équation de l'éclaté stricte $C$ après division par $y^{2}$.

\subsubsection{Singularités homogènes}

Ce procédé permet aussi de simplifier les singularités de feuilletages holomorphes. Prenons le cas d'une singularité $\mathcal{F}$ définie par une équation différentielle homogène du premier ordre :

$$
\frac{d y}{d x}=-\frac{P_{n}(x, y)}{Q_{n}(x, y)},
$$

$P_{n}$ et $Q_{n}$ étant des polynômes homogènes de même degré, disons $n \geq 2$, sans facteur commun. Le feuilletage $\mathcal{F}$ défini par cette équation se relève, en dehors du diviseur $D$, en un feuilletage $\widetilde{\mathcal{F}}$ que l'on prolonge au diviseur comme suit. Le feuilletage $\mathcal{F}$ est aussi défini par la forme différentielle $\omega=P_{n}(x, y) d x+Q_{n}(x, y) d y$. Dans la carte $V_{x}$, cette forme différentielle se relève en la forme

$$
\Pi^{*} \omega=x^{p}\left[\left(P_{n}(1, t)+t Q_{n}(1, t)\right) d x+Q_{n}(1, t) x d t\right] .
$$

Là, plusieurs situations sont possibles.

Si le polynôme $x P_{n}(x, y)+y Q_{n}(x, y)=x^{p+1}\left[P_{n}(1, t)+t Q_{n}(1, t)\right]$ est identiquement nul, alors $\widetilde{\mathcal{F}}$ est encore défini par $d t$ dans cette carte, ce qui permet de le prolonger au diviseur. En redescendant par $\Pi$ dans les coordonnées $(x, y)$, on reconnait le feuilletage défini par $x d y-y d y$, c'està-dire par les niveaux de la fonction méromorphe $\frac{y}{x}$.

Sinon, décomposons le polynôme $x P_{n}(x, y)+y Q_{n}(x, y)=\prod_{k=1}^{n+1}\left(y-t_{k} x\right)$ où $t_{k} \in \mathbb{C}$ pour $k=1, \ldots, n+1$ et supposons que ces racines sont deux à deux distinctes. On a supposé ici le polynôme unitaire et non divisible par $y$, ce qui est possible après un changement linéaire de coordonnées. Alors le feuilletage $\widetilde{\mathcal{F}}$ est encore défini dans la carte $V_{x}$ par la 1-forme :

$$
\left(\prod_{k=1}^{p+1}\left(t-t_{k}\right)\right) d x+Q_{n}(1, t) x d t
$$

qui ne s'annule qu'aux points $(x, t)=\left(0, t_{k}\right)$ pour $k=1, \ldots, n+1$ (car $P_{n}$ et $Q_{n}$ n'ont pas de facteur commun). L'équation différentielle se résout en décomposant en éléments simples :

$$
\frac{Q_{n}(1, t)}{P_{n}(1, t)+t Q_{n}(1, t)}=\frac{d x}{x}-\sum_{k=1}^{n+1} \alpha_{k} \frac{d t}{t-t_{k}}
$$


avec $\alpha_{k} \in \mathbb{C}^{*}$ pour $k=1, \ldots, n+1$ et $\sum_{k=1}^{n+1} \alpha_{k}=1$. La singularité de $\widetilde{\mathcal{F}}$ au point $\left(0, t_{k}\right)$ est définie par la 1-forme holomorphe

$$
\left(t-t_{k}\right) d x-\left(\alpha_{k}+\left(t-t_{k}\right) \sum_{l \neq k} \frac{\alpha_{l}}{t-t_{l}}\right) x d\left(t-t_{k}\right) .
$$

En particulier, si aucun des $\alpha_{k}$ n'est dans $\mathbb{Q}_{\geq 0}$, alors toutes les singularités de $\widetilde{\mathcal{F}}$ sont réduites : on dit dans ce cas que le feuilletage singulier $\widetilde{\mathcal{F}}$ est réduit.
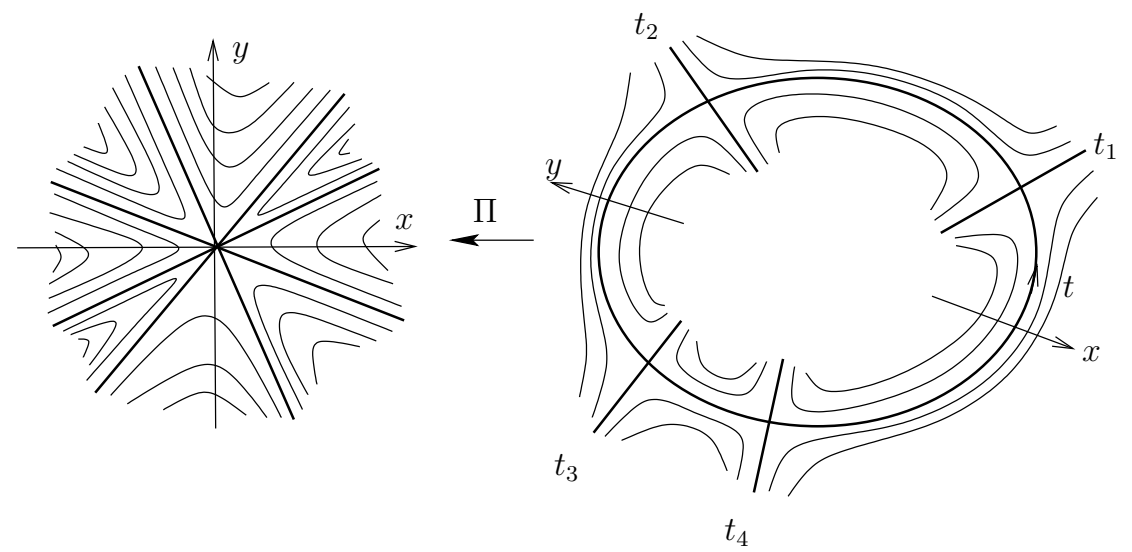

Figure 7.2 - Éclatement d'une singularité homogène

Exercice 7.1. Considérons une perturbation de la forme précédente :

$$
\omega=P_{n}(x, y) d x+Q_{n}(x, y) d y+\{\text { termes d'ordre }>n\} .
$$

Éclater la singularité 0 et vérifier que le feuilletage éclaté est réduit dès lors que $P_{n}(x, y) x+Q_{n}(x, y) y \not \equiv 0$ et $\alpha_{k} \notin \mathbb{Q} \geq 0$. En déduire, dans ce cas, l'existence de $n+1$ courbes invariantes lisses et deux-à-deux transverses pour $\mathcal{F}_{\omega}$.

\subsubsection{Singularités cuspidales}

Considérons le feuilletage $\mathcal{F}$ défini par

$$
\omega=d\left(y^{2}-x^{3}\right)+\Delta(x, y)(2 x d y-3 y d x), \quad \Delta(0,0)=0
$$


pour une fonction $\Delta \in \mathbb{C}\{x, y\}$ s'annulant à l'origine. Le cusp $C$ d'équation $F(x, y)=y^{2}-x^{3}$ est visiblement une courbe invariante pour $\mathcal{F}$. Après éclatement de l'origine, le feuilletage est défini, dans la carte $(x, t)$, par :

$$
\left(2 t^{2}-3 x-t \Delta(x, x t)\right) d x+(2 x t+2 x \Delta(x, x t)) d t
$$

et dans la carte $(T, y)$ par :

$$
\left(-3 T^{2} y^{2}-3 y \Delta(T y, y)\right) d T+\left(2-3 T^{3} y-T \Delta(T y, y)\right) d y
$$

Alors que, dans la seconde carte, le feuilletage est régulier le long du diviseur, une singularité apparait dans la première carte au point $(x, t)=(0,0)$. Cette singularité n'est pas réduite car elle est du type $x d x+\cdots=0$, ou encore parce qu'elle admet à la fois le diviseur $D$ et l'éclaté strict du cusp $\widetilde{C}$ d'équation $\widetilde{F}(x, t)=t^{2}-x$ comme courbes invariantes (la courbe invariante d'une singularité réduite est toujours un croisement normal, ou lisse dans le cas du nœud-col).

Nous allons procéder à un deuxième éclatement, à savoir l'éclatement de la singularité $(x, t)=(0,0)$. Pour cela, nous introduisons deux nouvelles cartes :

$$
\mathbb{C}^{2} \rightarrow V_{x} ;(u, t) \mapsto(u t, t) \quad \text { et } \quad \mathbb{C}^{2} \rightarrow V_{x} ;(x, U) \mapsto(x, U x)
$$

qui viennent remplacer la carte $(x, t)$ avec les nouveaux changements de carte :

$$
(x, U)=\left(u t, \frac{1}{u}\right) \quad \text { et } \quad(T, y)=\left(\frac{1}{t}, u t^{2}\right)
$$

En composant ces deux éclatements, on a finalement remplacé l'origine de $\mathbb{C}^{2}$ par deux courbes projectives, la première d'équation $u=0$ ou $y=0$ paramétrée par la variable projective $t=\frac{1}{T}$ et la seconde d'équation $t=0$ ou $x=0$ paramétrée par la variable projective $u=\frac{1}{U}$. Ces deux courbes s'intersectent transversalement au point $(u, t)=(0,0)$.

Le feuilletage relevé sur cette nouvelle variété est défini, dans la carte $(u, t)$, par :

$$
\left(2 t^{2}-3 u t-t \Delta\left(u t, u t^{2}\right)\right) d u+\left(4 u t-3 u^{2}+u \Delta\left(u t, u t^{2}\right)\right) d t
$$

et dans la carte $(x, U)$ par :

$$
\left(4 x U^{2}-3+U \Delta\left(x, x^{2} U\right)\right) d x+\left(2 x^{4} U+2 x \Delta\left(x, x^{2} U\right)\right) d U
$$

Le feuilletage obtenu admet les deux diviseurs comme feuilles et n'est singulier qu'à leur intersection où il admet comme troisième courbe invariante l'éclaté strict du cusp qui a pour équation $t-u=0$.

Un troisième éclatement est nécessaire (et en fait suffisant) en ce point pour réduire le feuilletage. La nouvelle variété $M$ et sa projection $\Pi: M \rightarrow$ 


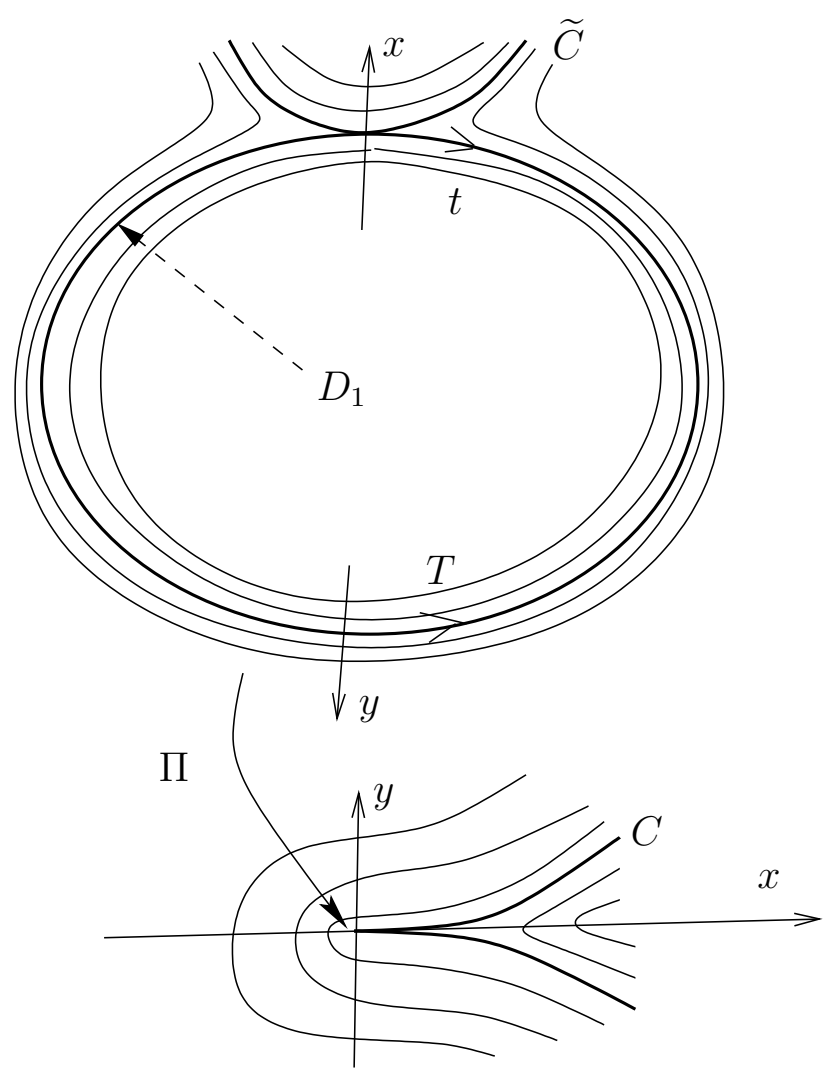

Figure 7.3 - Premier éclatement du cusp 


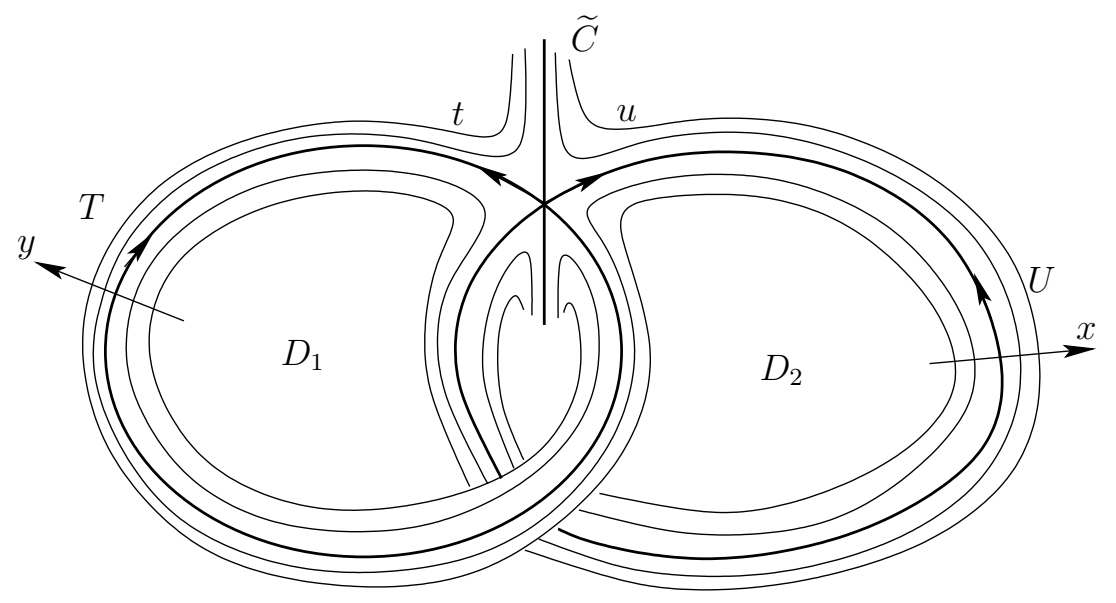

Figure 7.4 - Deuxième éclatement du cusp

$\mathbb{C}^{2}$ sont alors définies par quatre cartes :

$$
\Pi:\left\{\begin{array}{rlc}
(T, y) & \mapsto & (T y, y) \\
(V, t) & \mapsto & \left(V t^{2}, V t^{3}\right) \\
(u, v) & \mapsto & \left(u^{2} v, u^{3} v^{2}\right) \\
(x, U) & \mapsto & \left(x, x^{2} U\right)
\end{array}\right.
$$

et les changements de cartes :

$$
(V, t)=\left(T^{3} y, \frac{1}{T}\right), \quad(u, v)=\left(V t, \frac{1}{V}\right) \quad \text { et } \quad(x, U)=\left(u^{2} v, \frac{1}{u}\right) .
$$

La variété $M$ est construite en remplaçant l'origine de $\mathbb{C}^{2}$ par les 3 courbes rationnelles :

$$
\left\{\begin{array}{lll}
D_{1}:=\{y=V=0\} & \text { paramétrée par la variable } & t=\frac{1}{T} \\
D_{2}:=\{v=x=0\} & \text { paramétrée par la variable } & u=\frac{1}{U} \\
D_{1}:=\{t=u=0\} & \text { paramétrée par la variable } & v=\frac{1}{V}
\end{array}\right.
$$

s'intersectant transversalement aux points $p_{1}=D_{1} \cap D_{3}$ et $p_{2}=D_{2} \cap D_{3}$ définis respectivement par $(V, t)=(0,0)$ et $(u, v)=(0,0)$ et dont la réunion $D=D_{1} \cup D_{2} \cup D_{3}$ est appelé diviseur (exceptionnel) ou arbre (de réduction). L'éclaté strict $\widetilde{C}$ du cusp a pour équation $u=1$ : c'est une courbe lisse intersectant transversalement le diviseur au point $p_{3}=\widetilde{C} \cap D_{3}$ d'équation $(u, v)=(1,0)$.

Le feuilletage $\widetilde{\mathcal{F}}$ est donné dans la carte $(V, t)$ par :

$$
\left(2 t-3 V t-\Delta\left(V t^{2}, V t^{3}\right)\right) d V+\left(6 V-6 V^{2}\right) d t
$$


et dans la carte $(u, v)$ par :

$$
\left(6 v^{2}-6 v\right) d u+\left(4 u v-3 u+\Delta\left(u^{2} v, u^{3} v^{2}\right)\right) d v
$$

Ce feuilletage est singulier précisément aux points $p_{1}, p_{2}$ et $p_{3}$ et ces singularités sont réduites, données par :

$$
\left\{\begin{array}{ccc}
t d V+3 V d t & \text { au point } & p_{1} \\
2 v d u+u d v & \text { au point } & p_{2} \\
6(v-1) d u+u d(v-1) & \text { au point } & p_{3}
\end{array}\right.
$$

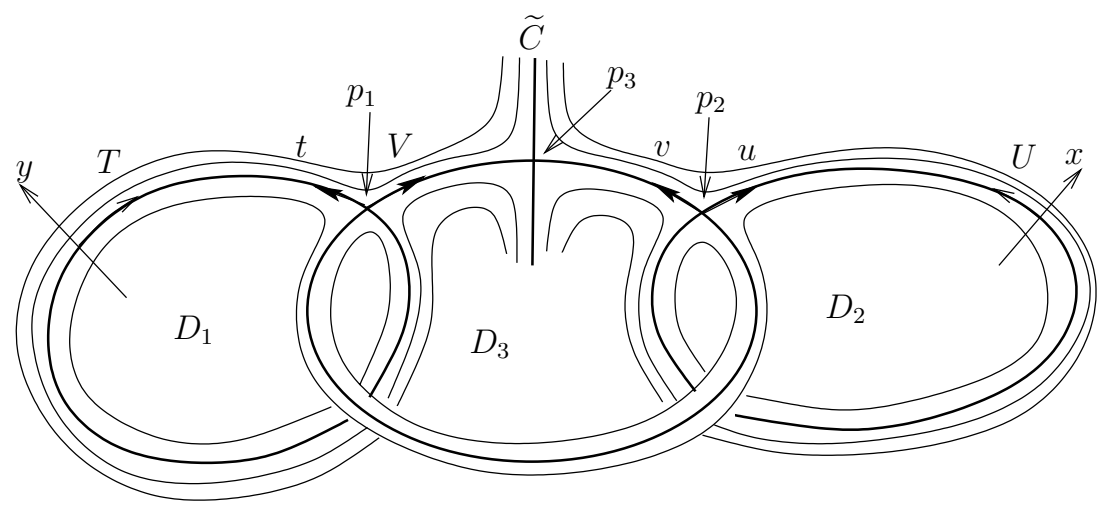

Figure 7.5 - Troisième éclatement du cusp

\subsubsection{Le Théorème de Seidenberg}

Si la réduction des singularités de feuilletages par éclatements était bien connue et largement utilisée au début du 20ème siècle, par exemple chez Dulac, l'algorithme général n'a été écrit qu'en 1968 par Seidenberg dans [201].

Soit $\mathcal{F}$ le feuilletage singulier défini au voisinage de $0 \in \mathbb{C}^{2}$ par un germe de 1 -forme holomorphe $\omega=f(x, y) d x+g(x, y) d y$. Après un éclatement $\Pi: M \rightarrow \mathbb{C}^{2}$ de l'origine, le feuilletage $\widetilde{\mathcal{F}}$ défini par $\Pi^{*} \omega$ se prolonge le long du diviseur exceptionnel $D=\Pi^{-1}(0)$ en divisant $\Pi^{*} \omega$ dans chaque carte par une équation convenable du divideur exceptionnel. Considérons maintenant une suite d'applications

$$
\cdots \rightarrow M_{k} \rightarrow M_{k-1} \rightarrow \cdots \rightarrow M_{1} \rightarrow M_{0}=\mathbb{C}^{2}
$$

où $\pi_{k+1}: M_{k+1} \rightarrow M_{k}$ est l'éclatement d'un point $p_{k}$ du diviseur exceptionnel $\Pi_{k}^{-1}(0), \Pi_{k}=\pi_{k} \circ \cdots \pi_{0}$, qui est d'un des types suivants 
— un point singulier non réduit pour le feuilletage relevé $\mathcal{F}_{k}=\Pi_{k}^{*} \mathcal{F}$,

- un point de tangence isolée de $\mathcal{F}_{k}$ avec une composante irréductible de $\Pi_{k}^{-1}(0)$.

Bien sûr, cette suite n'est pas unique : elle dépend à chaque étape du point $p_{k}$ choisi parmi ceux qui satisfont l'une ou l'autre des deux propriétés précédentes. Cet algorithme s'arrête dès que l'on ne peut plus trouver de point $p_{k}$ satisfaisant l'une ou l'autre des deux propriétés; on dit alors que l'application totale $\Pi_{k}: M_{k} \rightarrow \mathbb{C}^{2}$ est une réduction de la singularité $\mathcal{F}$. Le lecteur pourra trouver à la fin de [140] une démonstration de

Théorème 7.2 (Seidenberg). Toute suite construite comme au-dessus s'arrête en un temps fini. L'application $\Pi: M \rightarrow \mathbb{C}^{2}$ ainsi obtenue satisfait :

- $\Pi$ induit un isomorphisme $M \backslash \Pi^{-1}(0) \rightarrow \mathbb{C}^{2} \backslash\{0\}$,

- le diviseur exceptionnel au-dessus de 0 est une courbe nodale connexe non cyclique dont les composantes irréductibles sont lisses et rationnelles

$$
D=\Pi^{-1}(0)=D_{1} \cup D_{2} \cup \cdots \cup D_{k},
$$

- le feuilletage relevé $\widetilde{\mathcal{F}}=\Pi^{*} \mathcal{F}$, défini par $\widetilde{\omega}=\Pi^{*} \omega$, n'a que des singularités réduites le long de $D$,

- toute composante $D_{i}$ est ou bien une courbe invariante pour $\widetilde{\mathcal{F}}$, ou bien en tout point transverse $\grave{a} \widetilde{\mathcal{F}}$.

$L$ 'application $\Pi: M \rightarrow \mathbb{C}^{2}$ est indépendant de la suite d'éclatements choisie et elle est minimale dans le sens suivant : toute autre application $\widetilde{\Pi}: \widetilde{M} \rightarrow$ $\mathbb{C}^{2}$ satisfaisant aux propriétés précédentes se factorise $\widetilde{\Pi}=\Phi \circ \Pi$ via une application holomorphe $\Phi: \widetilde{M} \rightarrow M$.

On dit que la singularité $\mathcal{F}$ est dicritique si un au moins des projectifs est transverse au feuilletage après réduction.

Toute courbe invariante de $\mathcal{F}$ se relève via $\Pi: M \rightarrow \mathbb{C}^{2}$ en une courbe invariante $\widetilde{C}$ pour $\mathcal{F}$, à savoir l'éclaté stricte de $C$. Réciproquement, toute courbe invariante irréductible $\widetilde{C}$ qui n'est pas contenue dans le diviseur exceptionnel $D$ descend sur une courbe $C$ invariante pour $\mathcal{F}$.

Lorsque $\mathcal{F}$ est dicritique, chaque composante irréductible du diviseur qui est transverse à $\mathcal{F}$ donne naissance à un pinceau de courbes invariantes pour $\mathcal{F}$. C'est typiquement ce qui arrive lorsque $\mathcal{F}$ admet une intégrale première méromorphe.

Lorsque $\mathcal{F}$ n'est pas dicritique, la courbe invariante (resp. formelle) de $\widetilde{\mathcal{F}}$ est la réunion finie des composantes irréductibles du diviseur exceptionnel $D$ et des courbes invariantes (resp. formelles) transverses à $D$ attachées à ses points singuliers : les singularités réduites ont une courbe invariante nodale; chacune d'elle donne naissance à au plus une branche transverse à $D$.

Que $\mathcal{F}$ soit dicritique ou non, on voit que la réduction de $\mathcal{F}$ nécessite au moins la désingularisation de sa courbe invariante formelle. En général, 
c'est insuffisant comme le montre l'exemple 7.5. Cependant, on trouve dans [37] le

Théorème 7.3 (Camacho-Lins-Neto-Sad). Soit $\mathcal{F}$ une singularité de feuilletage, $\Pi: M \rightarrow \mathbb{C}^{2}$ l'application de réduction et $\widetilde{\mathcal{F}}$ le feuilletage réduit sur M. Si $\tilde{\mathcal{F}}^{\prime}$ 'a pas de singularité de type nœud-col le long du diviseur exceptionnel $D$, alors $\Pi$ est en fait l'application minimale désingularisant la courbe invariante de $\mathcal{F}$.

Dans [37], une telle singularité est appelée courbe généralisée. Puisque le procédé de réduction d'une courbe est un invariant topologique, il en va de même du procédé de réduction d'une courbe généralisée. On ne sait toujours pas à ce jour si deux singularités de feuilletages topologiquement conjuguées ont même réduction (en présence de nœud-cols). En dimension supérieure, les stratégies de réduction des singularités de feuilletages sont plus subtiles et nous renvoyons à [41].

Exemple 7.4. Les nœuds rationnels linéaires :

$$
\omega=p x d y-q y d x \quad \text { avec } \quad p, q \in \mathbb{N}^{*}
$$

ne sont pas des singularités réduites. Dans le cas radial, c'est-à-dire lorsque $(p, q)=(1,1)$, le feuilletage est régulier, transverse au diviseur exceptionnel après un éclatement. Lorsque $p=1$ et $q>1$, le feuilletage est réduit après $q$ éclatements : les $q-1$ premières composantes $D_{1}, \ldots, D_{q-1}$ (qui se suivent comme une chaîne) sont tangentes au feuilletage et la dernière $D_{q}$ est dicritique (i.e. transverse); les singularités sont toutes des cols avec intégrale première holomorphe situées aux coins $D_{i} \cup D_{i+1}, i=1, \ldots, q-2$, et une sur la partie lisse de $D_{1}$. Enfin, lorsque $p, q>1$ (on les suppose premiers entre eux), le diviseur exceptionnel est encore une chaîne après réduction avec une composante dicritique et un col à chaque extrémité. Le nombre d'éclatements, majoré par $p+q$, est le nombre d'étapes nécessaires dans l'algorithme d'Euclide appliqué à la paire $(p, q)$.

Exemple 7.5. Les singularités de Poincaré-Dulac

$$
\omega_{n}:=x d y-\left(n y+x^{n}\right) d x, \quad n \in \mathbb{N}^{*}
$$

ne sont pas réduites. Leur réduction est la même que pour les cols rationnels de type $(1, n)$ (voir exemple 7.4) excepté que le feuilletage réduit est tangent à tout le diviseur. La singularité située au croisement $D_{n-1} \cap D_{n}$ est le noud-col $\omega_{p, \lambda}$ (voir section 6.4) avec $(p, \lambda)=(1,-1)$, la variété centrale étant contenue dans $D_{n}$. Toutes les autres singularités, situées aux autres croisements ainsi que sur la partie lisse de $D_{1}$, sont des cols résonants (tous normalisables, i.e. d'invariants de Martinet-Ramis triviaux). 
En particulier, la courbe invariante (y compris formelle) du feuilletage défini par $\omega_{n}=0$ est lisse, donnée par $C=\{x=0\}$. Notons que la forme

$$
\frac{\omega_{n}}{x^{n+1}}=d\left(\frac{y}{x^{n}}\right)-\frac{d x}{x}
$$

est fermée.

Exemple 7.6. Le feuilletage défini par

$$
\omega=\left(2 y^{2}+x^{3}\right) d x-2 x y d y
$$

a pour intégrale première la fonction méromorphe $H(x, y)=\frac{y^{2}-x^{3}}{x^{2}}$. Après un éclatement, le feuilletage devient régulier, mais il n'est pas transverse au diviseur : il a une tangence simple au point d'attache du cusp. La réduction complète s'obtient après deux éclatements supplémentaires, comme dans la section 7.1.2. En reprenant les notations de cette section, le diviseur $D_{1}$ est dicritique, les deux autres $D_{2}$ et $D_{3}$ sont invariants et il y a deux cols, un sur $D_{3}$ et l'autre au coin $D_{2} \cap D_{3}$.

\subsection{La formule de l'indice}

Afin de motiver la définition de l'indice de Camacho-Sad, commençons par un exemple. Le feuilletage linéaire défini par $x d y-\alpha y d x$ a pour holonomie, le long de l'axe horizontal, $f(y)=a y$ où $a=e^{2 i \pi \alpha}$; changer $\alpha$ en $\alpha+1$ ne modifiera pas l'holonomie. Géométriquement, le logarithme $\alpha$ de $a$ rend compte de la manière dont les feuilles "tournent autour" de l'axe horizontal le long d'un lacet entourant l'origine :

$$
\gamma:[0,1] \rightarrow\{y=0\} ; \theta \mapsto e^{2 i \pi \theta}
$$

en effet, ce lacet se relève, via la projection $(x, y) \mapsto x$, en

$$
\tilde{\gamma}:[0,1] \rightarrow \mathbb{C}^{2} ; \theta \mapsto\left(e^{2 i \pi \theta}, e^{2 i \pi \alpha \theta} y_{0}\right) .
$$

Dans la variable $y$, transverse à la courbe invariante, on obtient un chemin reliant le point $y_{0}$ au point $y_{1}=a y_{0}$ dont la classe d'homotopie à extrémités fixes dans le voisinage épointé de $y=0$ dépend effectivement de $\alpha$ : changer $\alpha$ en $\alpha+1$ augmentera le nombre de tours que fait ce chemin autour de $y=0$ d'une unité.

Plus généralement, soit $\mathcal{F}$ une singularité de feuilletage à l'origine de $\mathbb{C}^{2}$ (non nécessairement réduite) possédant l'axe $C=\{y=0\}$ comme courbe invariante, et notons $f(y)=a y+\cdots$ l'holonomie correspondante. En relevant le lacet $\gamma$ dans les feuilles, comme au-dessus, on obtient encore dans la variable $y$ un chemin reliant $y_{0}$ à $y_{1}=f\left(y_{0}\right) \sim a y_{0}$ pour tout point 
de départ $y_{0}$ suffisamment proche de 0 . Asymptotiquement, ce chemin est proche de

$$
[0,1] \rightarrow \mathbb{C}^{*} ; \theta \mapsto e^{2 i \pi \alpha \theta} y_{0}
$$

pour un choix convenable $\alpha$ du logarithme de $a$. Ce "nombre d'enlacement" $\alpha$ est l'indice de Camacho-Sad de $\mathcal{F}$ le long de $C$ au point singulier. Pour le calculer, notons que $\mathcal{F}$ est localement défini par une 1-forme holomorphe à singularité isolée $f(x, y) y d x+g(x, y) d y$, c'est-à-dire par une équation de la forme :

$$
\frac{d y}{y}=-\frac{f(x, y)}{g(x, y)} d x .
$$

La 1-forme méromorphe $\eta:=-\frac{f(x, y)}{g(x, y)} d x$ est holomorphe en dehors de $x=0$ lorsqu'on la restreint à $C$. L'invariant de Camacho-Sad est alors donné par :

$$
\alpha:=\frac{1}{2 i \pi} \int_{\gamma} \eta
$$

c'est le résidu de $\left.\eta\right|_{C}$ en $x=0$. On vérifie aisément que pour une singularité réduite de la forme $x d y-\alpha y d x+\cdots$, on retrouve l'invariant $\alpha$ défini par la partie linéaire. En particulier, lorsque $\mathcal{F}$ est un nœud-col dont $C$ est la variété forte, on obtient $\alpha=0$; par contre, si $C$ est la variété centrale, alors $\alpha=\lambda$ est l'invariant formel défini dans la section 6.4 : c'est par exemple le cas pour la forme normale formelle $\omega_{p, \lambda}=x^{p+1} d y-y\left(1+\lambda x^{p}\right) d x$. On peut encore définir l'invariant de Camacho-Sad le long d'une courbe $C$ invariante singulière en remplaçant la variable $y$ par une équation locale réduite de la courbe (voir [24], p.35).

Lorsque le feuilletage singulier $\mathcal{F}$ est défini au voisinage d'une courbe $C$ complète, et que cette courbe est invariante, la formule de l'indice nous dit que l'auto-intersection $C \cdot C$ de la courbe est égale à la somme des invariants de Camacho-Sad de $\mathcal{F}$ sur tous ses points singuliers le long de $C$ (voir [24], p.37). Expliquons ceci dans le cadre qui nous intéresse.

Considérons une singularité de feuilletage $\mathcal{F}$ non réduite et, après réduction, une composante irréductible $D$ du diviseur qui est invariante par $\widetilde{\mathcal{F}}$. En reprenant les notations de la section 7.3, l'égalité :

$$
f_{1} \circ \cdots \circ f_{n+1}=\text { identité }
$$

nous donne la contrainte suivante. Si $\alpha_{k}$ est l'invariant de Camacho-Sad de $\widetilde{\mathcal{F}}$ au point singulier $p_{k}$, alors $f_{k}(z)=e^{2 i \pi \alpha_{k}} z+\cdots$ et, en considérant la partie linéaire de l'égalité ci-dessus, il vient

$$
\alpha_{1}+\cdots+\alpha_{n+1} \in \mathbb{Z}
$$

Cet entier est alors donné par : 
Lemme 7.7 (Formule de l'indice). La somme des indices de CamachoSad de $\widetilde{\mathcal{F}}$ le long d'une composante irréductible $D$ invariante du diviseur exceptionnel est donnée par l'auto-intersection de D :

$$
\alpha_{1}+\cdots+\alpha_{p+1}=D \cdot D .
$$

En particulier, c'est un entier strictement négatif.

Rappelons brièvement que l'auto-intersection $D \cdot D$ est le nombre d'intersections de $D$ (comptés avec signes) avec une perturbation lisse $C^{\prime} \sim C$ générique. C'est aussi la première classe de Chern du fibré normal à $D$. Cet entier caractérise le type topologique du voisinage tubulaire de $D$. Après 1 éclatement, le diviseur exceptionnel $D$ est d'auto-intersection -1 ; cet invariant décroît d'une unité à chaque fois que l'on éclate un point de $D$. Il est donc facile de calculer l'auto-intersection $D_{i} \cdot D_{i}$ de chaque composante irréductible du diviseur exceptionnel après réduction d'une singularité.

Idée de preuve. Soit $u$ une coordonnée projective sur $D$ telle que toutes les singularités $p_{k}$ soient contenues dans le disque $\delta$ de centre $u=0$ et de rayon 1 , et soit $v$ une coordonnée transverse à $D$ au voisinage de $\Delta(D$ est défini par $\{v=0\}$ ). Par définition de l'indice, le lacet $\gamma$ qui borde le disque $\Delta$ se relève, dans les coordonnées $(u, v)$ en un lacet homotope à

$$
\tilde{\gamma}:[0,1] \rightarrow \mathbb{C}^{2} ; t \mapsto\left(e^{2 i \pi t}, e^{2 i \pi \nu t} y_{0}\right)
$$

où $\nu=\alpha_{1}+\cdots+\alpha_{p+1} \in \mathbb{Z}$. Le feuilletage est régulier et donc trivial au voisinage du complémentaire $D \backslash \Delta$, un empilement de disques. Chaque relevé $\tilde{\gamma}$ borde un de ces disques. Choisissons-en un suffisamment proche du diviseur. On peut le compléter en une sphère $C^{\infty}$ avoisinant $D$ en recollant le disque défini par

$$
u \in \Delta \quad \text { et }\left\{\begin{array}{l}
v=y_{0} u^{\nu} \text { lorsque } \nu \geq 0 \\
v=y_{0} \bar{u}^{-\nu} \text { lorsque } \nu<0
\end{array}\right.
$$

(où $\bar{u}$ est le conjugué complexe) puis en lissant au voisinage de $\tilde{\gamma}$. La perturbation lisse $D^{\prime} \sim D$ ainsi construite intersecte $D$ exactement au point $u=v=0$ avec multiplicité $\nu$ (dans le second cas $\nu<0$, le disque défini au-dessus est anti-holomorphe et intersecte donc $D$ négativement). Finalement, ceci montre que $D \cdot D=D \cdot D^{\prime}=\nu$.

Rappelons que la formule de l'indice est un des ingrédients de la preuve du

Théorème 7.8 (Camacho-Sad). Toute singularité de feuilletage admet une courbe invariante. 
La démonstration consiste, si le feuilletage n'est pas dicritique (auquel cas c'est terminé), à prouver l'existence, après réduction, d'une singularité réduite $p$ sur une partie lisse du diviseur $D$ qui ne soit pas un nœudcol avec variété forte contenue dans $D$. En effet, la singularité possède alors automatiquement une séparatrice transverse qui redescend sur une séparatrice de la singularité $\mathcal{F}$ de départ. Il suffit pour cela de trouver une singularité $p$ dont l'indice $\alpha$ relatif à la composante irréductible $D_{i}$ $\mathrm{du}$ diviseur qui la contient soit non nul. La preuve est combinatoire. Une preuve constructive à l'aide d'un algorithme de type Newton-Puiseux a été donnée par José Cano dans [43].

Exercice 7.9. Montrer que pour $\alpha \in \mathbb{C}$ générique, la réduction du feuilletage d'intégrale première $H(x, y)=\left(y^{2}-x^{3}\right)\left(y^{3}-x^{2}\right)^{\alpha}$ s'obtient après 5 éclatements. Le diviseur exceptionnel est une chaîne $D_{2}^{\prime}, D_{3}^{\prime}, D_{1}, D_{3}, D_{2}$ symétrique par rapport à $D_{1}$, la partie droite (resp. gauche) correspondant à la désingularisation du cusp $y^{2}=x^{3}$ (resp. $\left.y^{3}=x^{2}\right)$. Le feuilletage éclaté possède 6 points singuliers : 4 sont aux intersections des composantes du diviseur exceptionnel, et 2 sont sur la partie lisse, précisément sur $D_{3}$ et $D_{3}^{\prime}$. Montrer que le feuilletage devient dicritique pour $\alpha=-\frac{2}{3},-1$ et $-\frac{3}{2}$, respectivement sur $D_{3}^{\prime}, D_{1}$ et $D_{3}$. Montrer que le feuilletage n'est pas réduit pour toute autre valeur $\alpha \in \mathbb{Q}_{<0}$.

On appellera arbre de réduction la donnée de la matrice $\left(D_{i} \cdot D_{j}\right)_{i, j=1, \ldots, k}$ d'intersection des composantes irréductibles du diviseur exceptionnel $D$ :

- $D_{i} \cdot D_{j}=0$ si $i \neq j$ et si $D_{i}$ et $D_{j}$ sont disjointes,

- $D_{i} \cdot D_{j}=1$ si $i \neq j$ et $D_{i}$ et $D_{j}$ s'intersectent,

- $D_{i} \cdot D_{i}=\nu$ est l'auto-intersection.

Cette donnée caractérise topologiquement le germe de voisinage $(M, D)$.

Plus généralement, on dit qu'un diviseur (réduit) $D$ sur une surface complexe $M$ peut être contracté sur un point, s'il existe $\pi: M \rightarrow M^{\prime}$ un morphisme entre variétés analytiques, et $p \in M^{\prime}$ un point, tels que $\pi^{-1}(p)=D$ et la restriction de $\pi$ à $M \backslash D \rightarrow M^{\prime} \backslash\{p\}$ est un isomorphisme. Alors on a d'après [88] (voir aussi [105, Theorem 4.9]) :

Théorème 7.10 (Grauert). Soit $M$ une surface complexe et $D \subset M$ un diviseur (réduit) nodal et connexe dont les composantes irréductibles $D_{1}, \ldots, D_{k}$ sont des courbes lisses. Alors $D$ peut être contracté sur un point si, et seulement si, la matrice d'intersection $\left(D_{i} \cdot D_{j}\right)_{i, j}$ des composantes irréductibles $D_{i}$ est définie négative.

Attention, dans l'énoncé précédent, $M^{\prime}$ est en général singulière en $p$. La caractérisation du cas lisse semble délicate (voir [105, Corollary 5.8]).

Exemple 7.11. Pour la réduction des courbes généralisées non dicritiques attachées au cusp $y^{2}=x^{3}$, l'arbre de réduction est décrit par la matrice 
d'intersection

$$
\left(\begin{array}{ccc}
-3 & 0 & 1 \\
0 & -2 & 1 \\
1 & 1 & -1
\end{array}\right)
$$

Exercice 7.12. Déterminer tous les arbres de réduction à moins de 4 composantes.

Exercice 7.13. Soient $\widetilde{\mathcal{F}}$ un germe de feuilletage singulier au voisinage d'un diviseur irréductible $D \simeq \overline{\mathbb{C}}$ invariant d'auto-intersection -1 , possédant exactement 2 singularités réduites le long de D. Montrer que c'est l'éclatement d'une singularité réduite.

Exercice 7.14. Considérons la surface singulière $S=\left\{z^{2}=\left(x+y^{2}\right)\left(x^{2}+\right.\right.$ $\left.\left.y^{7}\right)\right\}$. Après éclatments, on obtient une surface lisse $M \rightarrow S$ avec un diviseur exceptionel formé d'un cycle de trois courbes rationnelles, avec matrice d'interection :

$$
\left(D_{i} \cdot D_{j}\right)_{i, j}=\left(\begin{array}{ccc}
-2 & 1 & 1 \\
1 & -2 & 1 \\
1 & 1 & -3
\end{array}\right) .
$$

Supposons qu'il existe un feuilletage $\mathcal{F}$ sur $(M, D)$ tel que $D$ est totalement invariant, et $\mathcal{F}$ a exactement 3 points singuliers réduits et linéarisables aux coins de D. Etudier les contraintes sur les invariants de Camacho-Sad des 3 points singuliers et voir qu'il existe des configurations sans nœuds, et donc pour lesquels les séparatrices sont toutes contenues dans D. Camacho construit un tel feuilletage dans [28] pour montrer la non existence de séparatrices pour certains feuilletages sur les surfaces singulières : quand on contracte $D$, on obtient un feuilletage sur $D$ sans séparatrice au point singulier.

\subsection{Pseudo-groupes d'holonomie projective}

Étudions le feuilletage réduit $\widetilde{\mathcal{F}}$ au voisinage d'une composante irréductible $D$ de l'arbre de réduction qui est invariante pour $\widetilde{\mathcal{F}}$. Si l'on note $p_{1}, \ldots, p_{n+1} \in D$ les singularités de $\widetilde{\mathcal{F}}$ le long de $D$, alors le complément $D^{*}=D \backslash\left\{p_{1}, \ldots, p_{n+1}\right\}$ est une feuille de $\widetilde{\mathcal{F}}$. On peut calculer l'holonomie de $D^{*}$ sur un germe de transversale $T$ en $p_{0} \in D^{*}$ (voir section 5.3). Pour une raison d'indice (voir section 7.2), il y a toujours au moins une singularité sur chaque projectif tangent au feuilletage.

Pour cela, choisissons une collection de lacets :

$$
\gamma_{1}, \ldots, \gamma_{n+1}:[0,1] \rightarrow D \backslash\left\{p_{1}, \ldots, p_{n+1}\right\}
$$

basés en $p_{0}$ comme dans la figure ci-dessous : le lacet $\gamma_{k}$ est d'indice 1 autour de $p_{k}$ et d'indice -1 autour des autres singularités. 


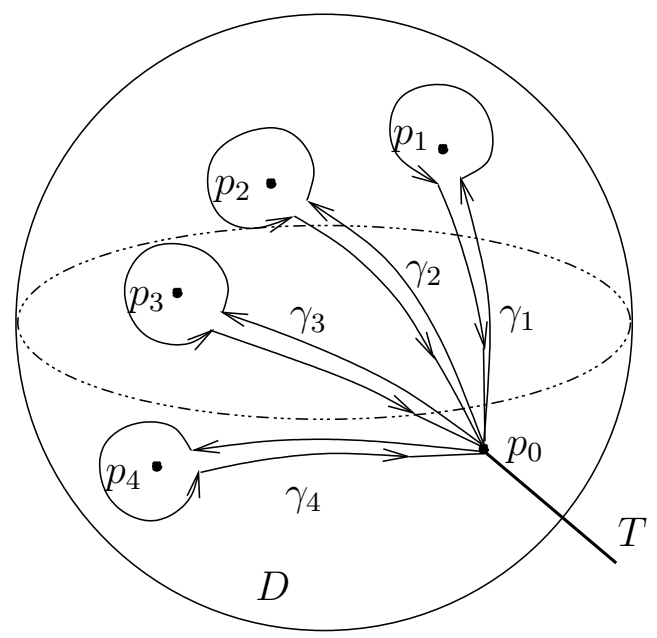

FigURE 7.6 - Holonomie projective

Choisissons une paramétrisation de $T$ par un voisinage ouvert $U \subset \mathbb{C}$ de l'origine $U \hookrightarrow T ; 0 \mapsto p_{0}$. Quitte à diminuer $T$ et $U$, chaque application d'holonomie $\varphi_{\gamma_{k}}$ définit, via la paramétrisation choisie, une transformation holomorphe inversible $f_{k}: U \rightarrow \mathbb{C}$ fixant 0 . Le pseudo-groupe $G$ engendré sur $U$ par ces transformations $f_{1}, \ldots, f_{n+1}$ est appelé pseudo-groupe d'holonomie projective associé à la composante $D$ du diviseur exceptionnel. Il n'est bien défini qu'à diminution près de $U$ et à conjugaison près par un difféomorphisme $\varphi: U \rightarrow U^{\prime} \subset \mathbb{C}$ fixant 0 . En particulier, le choix du point base $p_{0}$, de la transversale $T$ et des lacets $\gamma_{k}$ n'est pas important. Ce pseudo-groupe rend compte d'une partie de la dynamique transverse du feuilletage au voisinage de $D$ : chaque orbite est une partie de la trace d'une feuille sur la transversale $T$. Plus précisément, en reprenant l'étude des singularités réduites menée dans le chapitre 6 , on montre facilement les affirmations suivantes.

Lorsque les singularités $p_{1}, \ldots, p_{n+1}$ sont toutes réduites et aucune de type nœud ou nœud-col, alors on peut construire un voisinage tubulaire de $D$ sur lequel toute feuille autre que les courbes invariantes "verticales" des singularités vient couper $T$ et sa trace sur $T$ correspond exactement à une orbite. L'espace des feuilles autres que les séparatrices verticales s'identifie alors à l'espace des orbites du pseudo-groupe $G$.

Par contre, la présence d'un nœud-col dont $D$ serait la variété centrale ou encore d'un nœud parmis les singularités de $D$ amènerait des feuilles qui ne seraient pas vues par $T$ mais resteraient coïncées au voisinage de cette singularité. De même, la présence d'un nœud-col dont $D$ serait la 
variété forte ferait que certaines feuilles a priori distinctes du point de vue de $G$ viendraient se recoller au voisinage de celui-ci; si ce nœud-col admet, de plus, une variété centrale avec holonomie, ces recollements peuvent être "sauvages".

Remarquons que les générateurs $f_{k}$ du pseudo-groupe $G$ satisfont :

$$
f_{1} \circ \cdots \circ f_{n+1}=\text { identité }
$$

car le lacet $\gamma_{1} \cdots \gamma_{n+1}$ est homoptope à zéro dans $D \backslash\left\{p_{1}, \ldots, p_{n+1}\right\}$ et donc sans holonomie. Le pseudo-groupe $G$ est en fait engendré par $f_{1}, \ldots, f_{n}$.

Exercice 7.15. Considérons le cas d'une équation différentielle homogène :

$$
\frac{d y}{d x}=-\frac{P(x, y)}{Q(x, y)}
$$

dans le cas où elle se réduit après un éclatement (voir section 7.1.1). En remarquant que $\mathcal{F}$ est invariante par les homothéties $(x, y) \mapsto(a x, a y)$, $a \in \mathbb{C}^{*}$, déduire que le pseudo-groupe d'holonomie projective est du type (Lin). Expliciter ce pseudo-groupe en fonction des coefficients de $P$ et $Q$. En déduire qu'il existe un ouvert dense de couples $(P, Q)$ dans l'espace des couples de polynômes complexes à deux variables de degré $\leq n$ pour lesquels l'équation précédente a toutes ses feuilles denses exceptées les $n+1$ courbes invariantes.

Exemple 7.16. Revenons aux singularités cuspidales de la section 7.1.2. Rappelons qu'après réduction, le diviseur exceptionnel $D$ a 3 composantes irréductibles $D_{1}, D_{2}$ et $D_{3}$. Les deux premières n'ont qu'une singularité et, par conséquent, n'ont pas d'holonomie. Ces singularités sont des cols, ce qui implique, d'après le Théorème de Mattei-Moussu 6.3, que ces cols sont linéarisables et que les générateurs $f_{1}$ et $f_{2}$ du pseudo-groupe d'holonomie projective de $D_{3}$ sont périodiques. En considérant leur partie linéaire, donnée par la partie linéaire de $\widetilde{\mathcal{F}}$ aux points $p_{1}$ et $p_{2}$ (voir section 7.1.2), on déduit que :

$$
f_{1}^{\circ 3}=f_{2}^{\circ 2}=\text { identité. }
$$

Notons $G$ le sous-groupe de $\operatorname{Diff}(\mathbb{C}, 0)$ engendré par $f_{1}$ et $f_{2}$. D'après l'étude des deux premières leçons, on peut montrer que :

1. si $G$ est abélien, alors la dynamique de $G$ est de type (Fin),

2. si $G$ est résoluble non abélien, alors la dynamique est de type (Aff) et les orbites n'adhèrent qu'à 0 (c'est un cas particulier du 2ème item de la Proposition 4.21 avec a $a^{p}$ d'ordre 6),

3. enfin si $G$ n'est pas résoluble, alors la dynamique $G$ est sans secteur de Nakai, i.e. les orbites sont partout denses (application du Théorème 2.25). 
De plus, on peut montrer que $f_{3}$ est périodique si, et seulement si, on est dans les deux premières situations. Maintenant, en remarquant que les feuilles sont fermées au voisinage des projectifs $D_{1}$ et $D_{2}$, on déduit que tous les phénomènes dynamiques du feuilletage se lisent au voisinage du projectif $D_{3}$ puis que :

1. si $G$ est abélien, alors le feuilletage $\mathcal{F}$ admet une intégrale première holomorphe et toutes ses feuilles sont fermées,

2. si $G$ est résoluble non abélien, alors les feuilles de $\mathcal{F}$ adhèrent toutes au cusp et seulement au cusp,

3. enfin si $G$ n'est pas résoluble, alors toute feuille autre que le cusp est dense.

De plus, l'holonomie du cusp est triviale si, et seulement si, on est dans la situation 1 ou 2. Ces singularités ont été étudiées en 1983 par Robert Moussu pour répondre négativement à une conjecture de René Thom : il construit explicitement une singularité de type 2 ce qui montre qu'une singularité peut avoir des feuilles non analytiques (ici qui adhèrent au cusp) sans pour autant que la courbe invariante (ici le cusp) porte de l'holonomie. Une étude plus poussée du revêtement permet de montrer que les feuilles autres que le cusp sont des tores troués dans le cas 1 et des plans infiniment troués dans le cas 2, ces derniers s'obtenant en déroulant les tores troués. Pour plus de détails, nous renvoyons à [14\%, 74, 115, 116].

\subsection{Construction de singularités non réduites}

Considérons, pour commençer, une singularité de feuilletage de la forme

$$
\omega=P_{n}(x, y) d x+Q_{n}(x, y) d y+\Delta_{n}(x, y)(x d y-y d x)
$$

où $P_{n}$ et $Q_{n}$ sont des polynômes homogènes de degré $n \geq 2$ et $\Delta_{n} \in \mathbb{C}\{x, y\}$ s'annulant à l'ordre $n$ en 0 . En ce sens, $\omega$ est une perturbation radiale de la singularité homogène considérée dans la section 7.1.1. Nous supposerons $x P_{n}+y Q_{n}=\prod_{k=1}^{n+1}\left(y-t_{k} x\right)$ où les $t_{k} \in \mathbb{C}$ sont deux-à-deux distincts pour $k=1, \ldots, n+1$, de sorte que les droites $\left\{y=t_{k} x\right\}$ sont des courbes invariantes pour $\omega_{n}=P_{n}(x, y) d x+Q_{n}(x, y) d y$, et en fait pour $\omega$ puisque la perturbation est radiale. Alors, après un éclatement de l'origine, le diviseur exceptionnel $D$ est invariant et les singularités de $\widetilde{\mathcal{F}}$ sont les $n+1$ points $p_{k}=\left(0, t_{k}\right)$ dans la carte $(x, t)$; au voisinage de $p_{k}$, le feuilletage est donné par $\left(t-t_{k}\right) d x-\alpha_{k} x d\left(t-t_{k}\right)+\cdots$ où

$$
\frac{Q_{n}(1, t)}{P_{n}(1, t)+t Q_{n}(1, t)}=\frac{d x}{x}-\sum_{k=1}^{n+1} \alpha_{k} \frac{d t}{t-t_{k}}
$$


On note que la relation de Camacho-Sad $\sum_{k=1}^{n+1} \alpha_{k}=-1$ est satisfaite. Un point base $p_{0}$ et un système de générateurs $\gamma_{k}$ du groupe fondamental de $D^{*}=D \backslash\left\{p_{1}, \ldots, p_{n+1}\right\}$ étant fixés comme dans la section 7.3, la représentation d'holonomie permet de définir une "correspondance de Riemann-Hilbert non linéaire"

$$
\Delta_{n} \mapsto\left(\varphi_{1}, \ldots, \varphi_{n+1}\right)
$$

où $\varphi_{k}(z)=e^{2 i \pi \alpha_{k}} z+\cdots$ et $\varphi_{1} \circ \cdots \circ \varphi_{n+1}=$ identité. Le problème de Riemann-Hilbert, dans ce contexte, est de déterminer quelles représentations sont ainsi réalisées lorsque $\Delta$ décrit toutes les séries s'annulant à l'ordre $k$. Une obstruction est que, lorsque $\alpha_{k} \geq 0$, la singularité $p_{k}$ est un nœud ou un nœud-col dont l'holonomie $\varphi_{k}$ est très spéciale, par exemple linéarisable si $\alpha_{k} \notin \mathbb{Q}$. Par contre, Alcides Lins Neto montre dans [110] le

Théorème 7.17 (Lins Neto). Si $\alpha_{k} \in \mathbb{C} \backslash \mathbb{R}^{+}$pour $k=1, \ldots, n+1$, alors toute représentation

$$
\left(\varphi_{1}, \ldots, \varphi_{n+1}\right) \in(\operatorname{Diff}(\mathbb{C}, 0))^{n+1}
$$

satisfaisant $\varphi_{k}^{\prime}(0)=e^{2 i \pi \alpha_{k}}$ et $\varphi_{1} \circ \cdots \circ \varphi_{n+1}=$ identité est l'holonomie projective d'une singularité de la forme (7.1).

Comme le remarque Yulij Ilyashenko dans [93], on peut construire des éléments $\varphi_{1}, \varphi_{2}, \varphi_{3} \in \operatorname{Diff}(\mathbb{C}, 0)$ tous trois non linéarisables satisfaisant $\varphi_{1} \circ \varphi_{2} \circ \varphi_{3}=$ identité tels que pour tout choix de $\alpha_{k}<0$ tels que $\varphi_{k}^{\prime}(0)=$ $e^{2 i \pi \alpha_{k}}$ on ait $\alpha_{1}+\alpha_{2}+\alpha_{3} \leq-2$.

Démonstration. L'hypothèse $\alpha_{k} \in \mathbb{C} \backslash \mathbb{R}^{+}$permet de réaliser $\varphi_{k}$ comme holonomie d'une singularité $\mathcal{F}_{k}$ de la forme

$$
\left(t-t_{k}\right) d x-\alpha_{k} x d\left(t-t_{k}\right)+\cdots \quad \text { avec }\{x=0\} \text { invariante }
$$

au voisinage de $p_{k}$. En effet, lorsque $\alpha_{k} \notin \mathbb{R}, \varphi_{k}$ est linéarisable par Konigs (Théorème 3.3) et est l'holonomie de la singularité linéaire $\left(t-t_{k}\right) d x-$ $\alpha_{k} x d\left(t-t_{k}\right)$; dans le cas $\alpha_{k}<0$, on applique le Théorème 6.8. Nous allons recoller ces modèles locaux pour construire un feuilletage global au voisinage de $D$. Pour cela, choisissons un recouvrement ouvert $D=$ $V_{0} \cup V_{1} \cup \cdots \cup V_{n+1}$ comme dans la figure ci-dessous ( $V_{0}$ est un épaississement de $D-V, V=V_{1} \cup \cdots \cup V_{n+1}$, n'intersectant aucun des $\left.\gamma_{k}\right)$.

On peut supposer $\mathcal{F}_{k}$ défini sur un voisinage de $V_{k}, k=1, \ldots, n+1$, quitte à faire une homothétie dans la variable $t-t_{k}$. On peut supposer de plus que l'holonomie de $\mathcal{F}_{k}$ calculée sur la transversale $T=\left\{t=t_{0}\right\}$ est précisément $\varphi_{k}$ dans la variable $x$. Sur $V_{0}$, choisissons par exemple le feuilletage $\mathcal{F}_{0}$ défini par $d\left(y-t_{0} x\right)=\left(t-t_{0}\right) d x+x d t$ qui est régulier au 


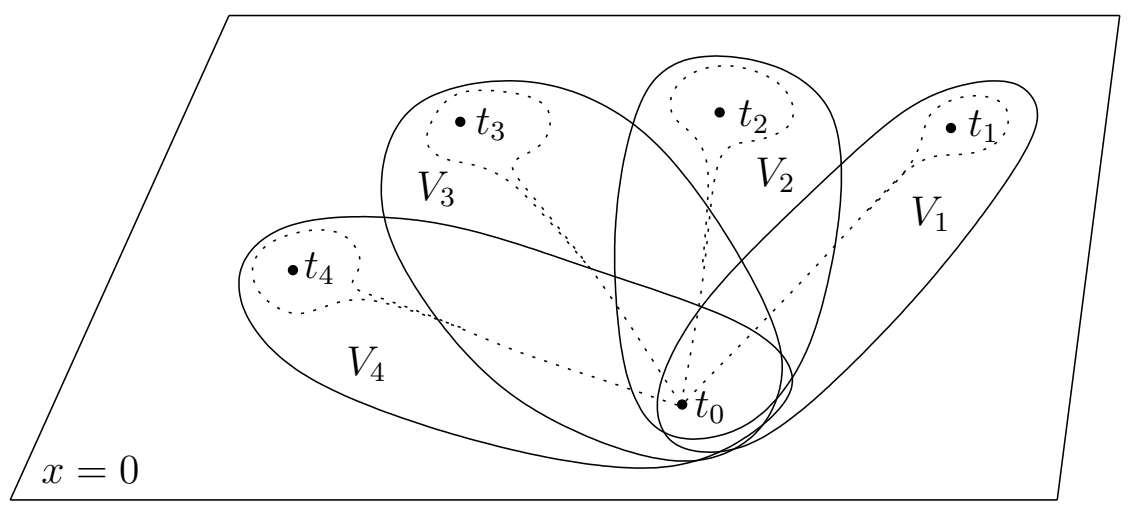

FIGURE 7.7 - Le recouvrement $V=V_{1} \cup \cdots \cup V_{n+1}$

voisinage de $D$ en dehors de $p_{0}$. Il existe pour tout $k=1, \ldots, n$ un (unique) germe de difféomorphisme

$$
\begin{gathered}
\Phi_{k}:\left(\mathbb{C}^{2}, V_{k} \cap V_{k+1}\right) \rightarrow\left(\mathbb{C}^{2}, V_{k} \cap V_{k+1}\right) ;(x, t) \mapsto\left(\phi_{k}(x, t), t\right), \\
\text { où } \quad\left\{\begin{array}{c}
\phi_{k}(0, t)=0 \\
\phi_{k}\left(x, t_{0}\right)=x
\end{array}\right.
\end{gathered}
$$

conjugant le feuilletage $\mathcal{F}_{k}$ au feuilletage $\mathcal{F}_{k+1}$; en effet, les deux feuilletages sont réguliers de feuille commune $\{x=0\}$ au voisinage de $V_{k} \cap V_{k+1}$ et cet ouvert est simplement connexe. Ceci permet d'ores et déjà de recoller les feuilletages $\mathcal{F}_{k}$ en un feuilletage $\mathcal{F}$ défini au voisinage de $V=V_{1} \cup \cdots \cup V_{n+1}$ dans un germe surface complexe $M$; la projection $(x, t) \mapsto t$ qui est invariante par les recollements $\Phi_{k}$ définit une projection $M \rightarrow V$ faisant de $M$ un fibré en disques. Par construction, le feuilletage $\mathcal{F}$ réalise la représentation d'holonomie que nous nous sommes donnée; il faut maintenant compléter le feuilletage sur un voisinage de $D$.

Il existe un germe de difféomorphisme

$$
\Phi_{0}:\left(\mathbb{C}^{2}, V_{0} \cap V\right) \rightarrow\left(M, V_{0} \cap V\right)
$$

qui commute aux projections et conjugue le feuilletage $\mathcal{F}_{0}$ au feuilletage $\mathcal{F}$ que nous venons de construire; la raison est la même qu'au-dessus à ceci près que $V_{0} \cap V$ n'est pas simplement connexe : on utilise ici que ni $\mathcal{F}_{0}$, ni $\mathcal{F}$ n'ont d'holonomie le long de $V_{0} \cap V$ (et notamment que $\varphi_{1} \circ \cdots \circ \varphi_{n+1}=$ identité). Finalement, en recollant $\mathcal{F}_{0}$ à $\mathcal{F}$ à l'aide de $\Phi_{0}$, on construit un fibré en disques $M \rightarrow D$ dont $D$ est une section globale; le feuilletage $\mathcal{F}$ est défini sur $M$, et $D$ en est une courbe invariante. Les singularités de $\mathcal{F}$ 
sont les points $p_{k}$ et la formule de l'indice (Lemme 7.7) nous dit que la classe de Chern de $D$ est -1 . D'après le Théorème de Grauert (voir [88]), $(M, D)$ est l'éclaté de $\left(\mathbb{C}^{2}, 0\right)$.

Dans la construction précédente, on peut choisir des modèles locaux dont la seconde courbe invariante est exactement $\left\{t=t_{k}\right\}$, de sorte qu'elle est contenue, après recollement, dans la fibration $M \rightarrow D$. Cette dernière définit un feuilletage singulier $\mathcal{G}$ sur $\left(\mathbb{C}^{2}, 0\right)$ qui doit être de la forme $x d y-$ $y d x+\cdots ;$ d'après le Théorème de linéarisation de Poincaré, $\mathcal{G}$ est linéaire dans de bonnes coordonnées holomorphes et les courbes invariantes de $\mathcal{F}$ sont redressées sur des droites. Par construction, on peut supposer, après transformation linéaire, que la courbe invariante est précisément

$$
\prod_{k=1}^{n+1}\left(y-t_{k} x\right)=x P_{n}+y Q_{n} .
$$

Alors la 1-forme $\omega$ définissant $\mathcal{F}$ sur $\left(\mathbb{C}^{2}, 0\right)$ se décompose en

$$
\omega=f\left(P_{n} d x+Q_{n} d y\right)+g(x d y-y d x) \quad \text { où } \quad\left\{\begin{array}{c}
f d x \wedge d y=\frac{\omega \wedge(x d y-y d x)}{x P_{n}+y Q_{n}} \\
g d x \wedge d y=\frac{\left(P_{n} d x+Q_{n} d y\right) \wedge \omega}{x P_{n}+y Q_{n}}
\end{array}\right.
$$

On vérifie aisément, du fait que les $n+1$ singularités sont non dégénérées après un éclatement, que $f(0) \neq 0$ et $g(0)=0$. En divisant $\omega$ par $f$, on obtient la forme désirée avec $\Delta=g / f$.

La méthode de recollement utilisée dans la preuve précédente se généralise sans difficulté à un arbre de réduction général. Par exemple, on peut montrer dans le cadre des singularités cuspidales (voir section 7.1.2 et l'exemple 7.16) que

Théorème 7.18 ([58]). Étant donnés $\left(f_{1}, f_{2}\right) \in(\operatorname{Diff}(\mathbb{C}, 0))^{2}$ satisfaisant

$$
f_{1}^{\circ 3}=f_{2}^{\circ 2}=\text { identité } \quad \text { avec } \quad\left\{\begin{array}{c}
f_{1}(z)=e^{\frac{2 i \pi}{3}} z+\cdots \\
f_{2}(z)=-z+\cdots
\end{array}\right.
$$

il existe une singularité cuspidale

$$
\omega=d\left(y^{2}-x^{3}\right)+\Delta(x, y)(2 x d y-3 y d x), \quad \Delta(0,0)=0
$$

dont l'holonomie projective est donnée par $\left(f_{1}, f_{2}\right)$ (voir exemple 7.16).

Idée de démonstration. On reconstruit d'abord le feuilletage au voisinage de $D_{3}$, réalisant la représentation d'holonomie donnée par $\left(f_{1}, f_{2}\right)$; on utilise pour cela les modèles locaux décrits à la fin de la section 7.1.2. Par la formule de l'indice, $D_{3}$ est de classe de Chern -1 dans le germe de surface construit, de sorte que l'on peut effondrer $D_{3}$ : on a maintenant un germe de feuilletage $\mathcal{F}_{2}$ sur $\left(\mathbb{C}^{2}, 0\right)$. Maintenant, on reconstruit le feuilletage au 
voisinage de $D_{2}$ comme dans la configuration de la figure 7.4 en utilisant $\mathcal{F}_{2}$ comme modèle local ; notons que la construction de la preuve du Théorème 7.17 n'utilise pas le fait que les singularités soient non dégénérées. Le diviseur $D_{2}$ est maintenant de classe de Chern -1 dans la surface construite et, en l'effondrant, on obtient un germe de feuilletage $\mathcal{F}_{1}$ sur $\left(\mathbb{C}^{2}, 0\right)$. Enfin, en utilisant celui-ci comme modèle local, on reconstruit le feuilletage $\mathcal{F}$ au voisinage de $D_{1}$ comme dans la configuration de la figure 7.3. Notons que l'on peut aussi construire directement le feuilletage $\mathcal{F}$ le long d'un diviseur $D=D_{1} \cup D_{2} \cup D_{3}$ en suivant la configuration de la figure 7.5 puis effondrer successivement les composantes $D_{3}, D_{2}$ puis $D_{1}$. Pour retrouver la forme $\omega$ de l'énoncé, on peut ou bien modifier la construction précédente de manière à construire simultanément la fibration $\mathcal{G}$ définie par $2 x d y-3 y d x$, ou encore utiliser le fait que la courbe invariante $C$, dont la paire de Puiseux $(2,3)$ est déterminée par sa réduction, se redresse analytiquement sur le cusp standart.

La construction du Théorème 7.17 s'adapte aussi aux feuilletages dicritiques. En effet, on a le

Lemme 7.19. Pour $k=1, \ldots, n+1$, on se donne un germe de feuilletage $\mathcal{F}_{k}$ défini au voisinage de $p_{k}=\left(0, t_{k}\right)$ dans $(x, t) \in \mathbb{C}^{2}$, les $p_{k}$ étant deux à deux distincts, et on suppose que $D=\{x=0\}$ n'est invariant par aucun des $\mathcal{F}_{k}$. Alors il existe un germe de feuilletage $\mathcal{F}$ sur $\left(\mathbb{C}^{2}, 0\right)$ tel qu'après 1 éclatement $\Pi:(M, D) \rightarrow\left(\mathbb{C}^{2}, 0\right)$, on ait (dans la carte $(x, t) \in \mathbb{C}^{2}$ avec les mêmes notations) :

- le diviseur $D$ est transverse au feuilletage éclaté $\widetilde{\mathcal{F}}$ excepté aux points $p_{k}$,

- il existe pour $k=1, \ldots, n+1$ un germe de difféomorphisme

$$
\Phi_{k}:\left(\mathbb{C}^{2}, p_{k}\right) \rightarrow\left(\mathbb{C}^{2}, p_{k}\right) ;(x, t) \mapsto(x, \phi(x, t)), \quad \phi(0, t)=t
$$

conjugant $\mathcal{F}_{k}$ à $\widetilde{\mathcal{F}}$.

La condition $\phi(0, t)=t$ a pour but d'avoir un contrôle sur le pseudogroupe du feuilletage construit, c'est-à-dire de prescrire la restriction des pseudo-groupes $G_{k}$ de $\mathcal{F}_{k}$ à la transversale globale $D$ (elle devient transverse au feuilletage après réduction des singularités $\mathcal{F}_{k}$ ).

Démonstration. La démonstration est la même que dans la preuve du Théorème 7.17 excepté que les applications de recollement entre les $V_{k}$ sont ici de la forme $(x, \phi(x, t))$ (transversalité au feuilletage). Pour que le diviseur $D$ soit d'auto-intersection -1 , on peut effectuer une chirurgie $a$ posteriori : au voisinage $V$ d'un cercle contenu dans $D$ et ne contenant pas de point singulier, disons le cercle unité $\{|t|=1\}$, le feuilletage est un fibré en disques qui se redresse sur $d t=0$; il suffit alors de procéder par chirurgie à l'aide d'une application de la forme $(x, t) \mapsto\left(x t^{\nu}, t\right)$ pour modifier l'auto-intersection $D \cdot D$ à volonté. 
Nous sommes maintenant en mesure de construire des singularités de feuilletage dont la réduction et l'holonomie sont arbitrairement riches. Dressons une liste des ingrédients qui nous permettront plus loin de donner une description quasi-complète du pseudo-groupe transverse pour une singularité de feuilletage générale. À chaque singularité $\mathcal{F}$, on sait maintenant associer :

1. Un arbre de réduction, c'est-à-dire le diviseur $D=D_{1} \cup \cdots \cup D_{k}$ muni de sa matrice d'intersection $\left(D_{i} \cdot D_{j}\right)_{i, j}$.

2. La partition $\{1, \ldots, n\}=I^{\text {inv }} \cup I^{\text {tran }}$ en composantes invariantes, indexées par $I^{\text {inv }}$ ou transverses, indexées par $I^{\text {tran }}\left(\operatorname{avec} D_{i} \cdot D_{j}=0\right.$ pour tous $i, j \in I^{\text {tran }}$ distincts).

3. Sur chaque composante $D_{i}$, la donnée d'une coordonnée projective $t_{i} \in \overline{\mathbb{C}}$ ainsi que sa valeur $t_{i, j}$ au point d'intersection $p_{i, j}=D_{i} \cdot D_{j}$ (lorsqu'elle est non vide).

4. Pour chaque composante invariante $D_{i}, i \in I^{\text {inv }}$, une collection (éventuellement vide) de singularités $p_{k}^{i} \in D_{i} \backslash\left\{p_{i, j}\right\}, k=1, \ldots, n_{i}$, deux à deux distinctes sur la partie lisse du diviseur; on note $t_{k}^{i}=t_{i}\left(p_{k}^{i}\right)$.

5. En chaque point $p_{i, j}=D_{i} \cap D_{j}, i, j \in I^{\text {inv }}$, un germe de feuilletage réduit $\mathcal{F}_{i, j}$ dont $D_{i} \cup D_{j}$ est la courbe invariante, donné par une 1forme $\omega_{i, j}$ dans les coordonnées $\left(t_{i}, t_{j}\right)$ (avec l'identification évidente entre $\omega_{i, j}$ et $\left.\omega_{j, i}\right)$.

6. En chaque point $p_{k}^{i} \in D_{i}, i \in I^{\text {inv }}$, un germe de feuilletage réduit $\mathcal{F}_{k}^{i}$ dont $D_{i}$ est une courbe invariante, donné par une 1 -forme $\omega_{i, j}$ dans les coordonnées $\left(t_{i}, z\right)$ (où $z$ est n'importe quelle équation locale réduite de $D_{i}$ ). Les indices de Camacho-Sad $\alpha_{i, j}$ et $\alpha_{k}^{i}$ le long de $D_{i}$ doivent satisfaire à la relation $\sum_{j} \alpha_{i, j}+\sum_{k} \alpha_{k}^{i}=D_{i} \cdot D_{i}$.

7. Pour chaque composante invariante $D_{i}, i \in I^{\text {inv }}$, une transversale $T_{i}$ en un point $p_{0}^{i} \in D_{i}^{*}=D_{i} \backslash\left\{p_{i, j}, p_{k}^{i}\right\}$ munie d'une coordonnée de référence $z_{i} \in(\mathbb{C}, 0)$.

8. Pour chaque point singulier $p_{k}^{i}\left(\operatorname{resp} . p_{i, j}\right)$ sur $D_{i}, i, j \in I^{\text {inv }}$, un lacet $\gamma_{k}^{i}\left(\right.$ resp. $\left.\gamma_{i, j}\right)$ basé en $p_{0}^{i}$ dans $D_{i}^{*}$, d'indice 1 autour de ce point et 0 autour des autres, donné dans la variable $t_{i}$; l'applications d'holonomie correspondante $\varphi_{k}^{i}:\left(T_{i}, p_{0}^{i}\right) \rightarrow\left(T_{i}, p_{0}^{i}\right)$ (resp. $\left.\varphi_{i, j}\right)$ donnée dans la variable $z_{i}$. Bien sûr, $\varphi_{k}^{i}$ (resp. $\varphi_{i, j}$ ) est (conjuguée à) l'holonomie de $\mathcal{F}_{k}^{i}$ (resp. $\mathcal{F}_{i, j}$ ) le long de $D_{i}$.

9. Pour chaque point $p_{i, j}$ intersectant une composante invariante $D_{i}$, $i \in I^{\text {inv }}$, avec une composante transverse $D_{j}, j \in I^{\text {tran }}$, un chemin $\delta_{i, j}$ dans $D_{i}^{*}$ joignant $p_{0}^{i}$ à $p_{i, j}$ et de l'application d'holonomie associée $\varphi_{i, j}:\left(T_{i}, p_{0}^{i}\right) \rightarrow\left(D_{j}, p_{i, j}\right)$, donnée dans les coordonnées $z_{i}$ et $t_{j}$.

Théorème 7.20 (Lins Neto). Réciproquement, étant donnés

$D_{i} \cdot D_{j}, I^{\mathrm{inv}}, I^{\operatorname{tran}}, t_{i}, t_{i, j}, t_{k}^{i}, \omega_{i, j} \omega_{k}^{i}, z_{i}, \gamma_{i, j}, \delta_{i, j}, \gamma_{k}^{i}, \varphi_{i, j}$ et $\varphi_{k}^{i}$ 
satisfaisant $1, \ldots, 9$ ci-dessus, il existe un germe de feuilletage singulier $\mathcal{F}$ dans $\left(\mathbb{C}^{2}, 0\right)$ et une suite d'éclatements $\Pi:(M, D) \rightarrow\left(\mathbb{C}^{2}, 0\right)$ tels que le feuilletage éclaté $\widetilde{\mathcal{F}}=\Pi^{*} \mathcal{F}$ réalise toutes ces données.

Attention, il se peut que $\Pi$ ne soit pas la réduction minimale de $\mathcal{F}$. 


\section{Chapitre 8}

\section{Structure transverse d'une singularité générale}

Nous allons décrire les pseudo-groupes d'holonomie que l'on obtient en recollant les dynamiques des différentes composantes du diviseur exceptionnel après réduction.

\subsection{Correspondances de Dulac}

Pour décrire le pseudo-groupe transverse d'une singularité de feuilletage $\mathcal{F}$, il nous faut notamment comprendre comment les différents pseudogroupes d'holonomie projective définis dans la section 7.3 sont connectés. En reprenant les notations de la section 7.4, on s'interesse au passage de la coordonnée $z_{i}$ à la coordonnée $z_{j}$ lorsque $D_{i}$ et $D_{j}$ s'intersectent avec $i, j \in S$. Ceci revient, étant donnée une singularité réduite possédant deux courbes invariantes, à étudier l'application de passage d'une transversale à une de ces courbes vers une transversale à l'autre courbe. Dans le cadre réel, ces applications sont bien définies en tant qu'homéomorphismes $\left(\mathbb{R}^{+}, 0\right) \rightarrow$ $\left(\mathbb{R}^{+}, 0\right)$ analytiques en dehors de 0 ; elles ont été étudiées par Henri Dulac dans son travail sur les cycles limites, relatif au $16^{\text {ème }}$ problème de Hilbert. Dans le cadre complexe, ces applications sont en général multiformes.

Pour la suite, la singularité réduite est définie dans un système de coordonnées $(x, y)$ au-delà du polydisque $\mathbb{D} \times \mathbb{D}$, les axes de coordonnées sont les séparatrices, les transversales sont :

$$
T_{x}:=\{y=1\} \quad \text { et } \quad T_{y}:=\{x=1\}
$$

paramétrées respectivement par $x$ et $y$ et l'application de Dulac complexe :

$$
\mathcal{D}: T_{x} \rightarrow T_{y}
$$


va maintenant être décrite pour chaque type de singularité réduite.

\subsubsection{Type $(\text { Fin })_{\text {col }}: \omega=p y d x+q x d y$ avec $p, q \in \mathbb{N}^{*}$ pre- miers entre eux}

L'intégrale première du feuilletage $H(x, y)=x^{p} y^{q}$ nous donne, en posant :

$$
H(x, 1)=H(1, \mathcal{D}(x))
$$

que l'application de Dulac $\mathcal{D}$ est une correspondance finie de type $(p: q)$ :

$$
\mathcal{D}: T_{x} \rightarrow T_{y} ; x \mapsto y=x^{p / q} .
$$

Lorsque $q=1$, cette application est holomorphe et c'est la seule situation, comme nous allons le voir, pour laquelle l'application de Dulac n'a pas de monodromie.

\subsubsection{Type $(\operatorname{Lin})_{\text {col }}$ et $(\operatorname{Lin})_{\text {hyp }}: \omega=x d y-\alpha y d x$ avec $\alpha \in \mathbb{C} \backslash\left(\mathbb{R}_{\geq 0} \cup \mathbb{Q}_{<0}\right)$}

L'intégrale première $H(x, y)=x^{-\alpha} y$ nous donne :

$$
\mathcal{D}: T_{x} \rightarrow T_{y} ; x \mapsto y=x^{-\alpha} .
$$

Pour la comprendre, le plus simple est de passer au revêtement universel

$$
\mathbb{H}_{\widetilde{z}} \rightarrow \mathbb{D}_{z} \simeq T_{z}^{*} ; \widetilde{z} \mapsto z=\exp (2 i \pi \widetilde{z})
$$

sur chaque transversale épointée $T_{z}^{*}=T_{z} \backslash\{z=0\}$, pour $z=x$ et $z=y$. L'application de Dulac $\mathcal{D}$ se relève par exemple en

$$
\widetilde{\mathcal{D}}: \mathbb{H}_{\widetilde{x}} \rightarrow \mathbb{H}_{\widetilde{y}} ; \widetilde{x} \mapsto \widetilde{y}=-\alpha \widetilde{x} .
$$

Les autres relèvements possibles se déduisent en composant par les automorphismes de revêtements : $\widetilde{\mathcal{D}}^{\prime}=\left(\Delta_{y}\right)^{\circ n} \circ \widetilde{\mathcal{D}} \circ\left(\Delta_{x}\right)^{\circ m}, m, n \in \mathbb{Z}$, où

$$
\Delta_{z}: \widetilde{z} \mapsto \widetilde{z}+1, \quad z=x, y .
$$

L'application $\widetilde{\mathcal{D}}$ est bien définie et on voit immédiatement que pour $\alpha \notin \mathbb{R}$, le domaine de définition de l'application de Dulac $\mathcal{D}$, sur $T_{x}$, va décroître au fur et à mesure que l'on va faire jouer la monodromie : ce n'est pas une application multiforme définie sur $\mathbb{H}_{\widetilde{x}}$ tout entier. Si l'on note $f$ et $g$ les holonomies respectives de l'axe des $x$ et des $y$ calculées sur les transversales $T_{x}$ et $T_{y}$, alors l'application de Dulac conjugue :

l'holonomie $\quad \widetilde{g}: \widetilde{x} \mapsto \widetilde{x}+\frac{1}{\alpha} \quad$ à la translation $\quad \Delta_{y}^{\circ(-1)}: \widetilde{y} \mapsto \widetilde{y}-1$ et la translation $\quad \Delta_{x}: \widetilde{x} \mapsto \widetilde{x}+1 \quad$ à l'holonomie $\quad \widetilde{f}^{\circ(-1)}: \widetilde{y} \mapsto \widetilde{y}-\alpha$.

Le fait que toutes les feuilles qui coupent $T_{x}$ coupent aussi $T_{y}$ se traduit ici par le fait qu'en tout point de $T_{x}$, il existe au moins une détermination de $\mathcal{D}$. On dira que la singularité ne disconnecte pas le feuilletage. 


\subsubsection{Type $(\operatorname{Lin})_{\text {nœud }}: \omega=x d y-\alpha y d x$ avec $\alpha \in \mathbb{R}_{\geq 0} \backslash \mathbb{Q}_{\geq 0}$}

Ici, contrairement aux situations précédentes, la singularité disconnecte totalement le feuilletage : aucune feuille passant par $T_{x}$ ne repasse par $T_{y}$ et vice-versa dès lors qu'on se restreint à un voisinage de l'origine : il n'y a pas d'application de Dulac pour de telles singularités.

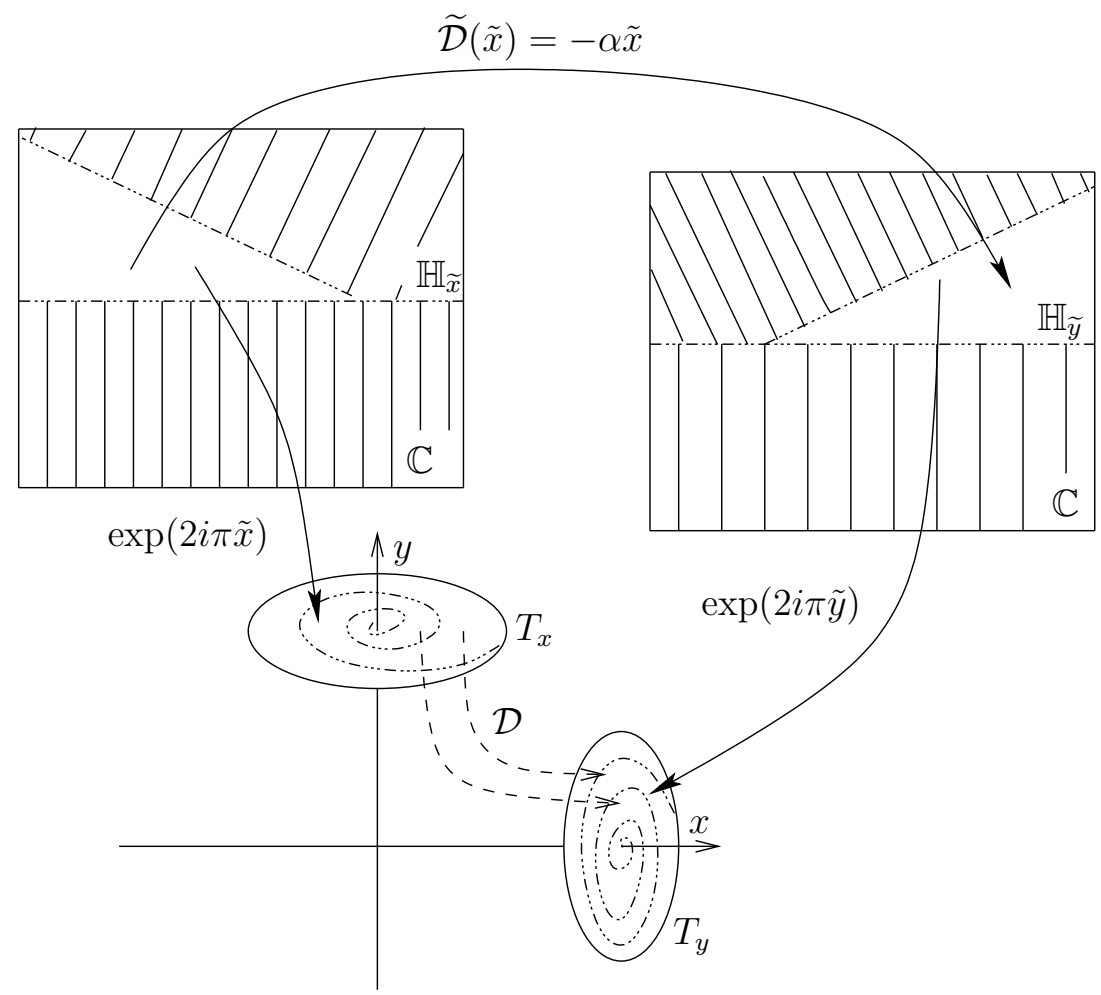

Figure 8.1 - Application de Dulac d'une singularité hyperbolique

\subsubsection{Singularités de type col : $\omega=x d y-\alpha y d x+\cdots$ avec $\alpha \in \mathbb{R}_{<0}$}

On commence par construire une détermination $\underline{\mathcal{D}}$ de l'application de Dulac comme suit. Étant donné un point $(x, 1)$ de $T_{x}$, son image par $\underline{\mathcal{D}}$ est le point $(1, y)=\varphi_{\gamma}(x, 1)$ où l'application d'holonomie $\varphi_{\gamma}$ est construite en relevant, suivant la fibration transverse $x=$ constante, le chemin composé du segment radial $\left[x, \frac{x}{|x|}\right]$ puis de l'arc de cercle ramenant, dans le sens direct, le point $\frac{x}{|x|}$ sur le point 1 dans la base (voir figure 8.2). L'applica- 
tion $\mathcal{D}$ s'obtient en prolongeant indéfiniment la détermination précédente à l'aide de l'identité suivante :

$$
\mathcal{D}\left(e^{2 i \pi} x\right)=f^{\circ(-1)} \circ \mathcal{D}(x)
$$

où $f$ désigne l'holonomie de l'axe des $x$.

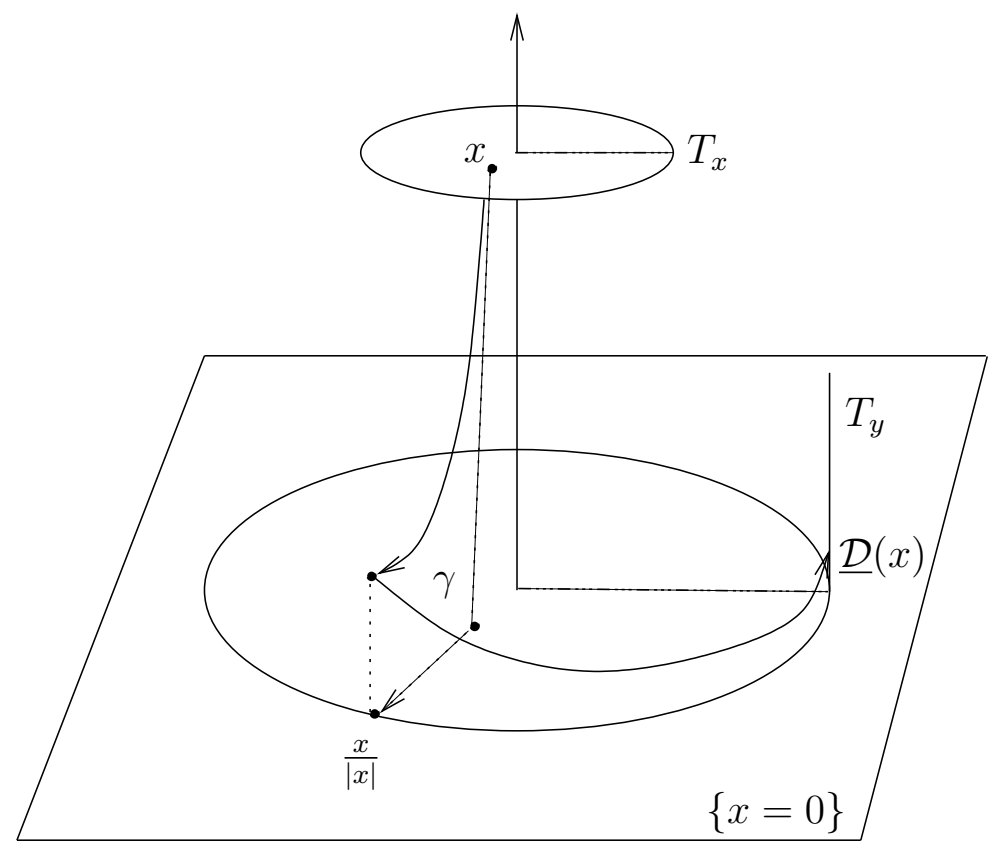

Figure 8.2 - Application de Dulac d'un col (non linéaire)

En inversant les rôles de $x$ et $y$ dans la construction précédente, on établit l'identité :

$$
\mathcal{D}^{\circ(-1)}\left(e^{2 i \pi} y\right)=g^{\circ(-1)} \circ \mathcal{D}^{\circ(-1)}(y)
$$

où $\mathcal{D}^{\circ(-1)}$ est l'application de passage de $T_{y}$ à $T_{x}$ et où $g$ désigne l'holonomie de l'axe des $y$. Les applications $\mathcal{D}$ et $\mathcal{D}^{\circ(-1)}$ sont inverses l'une de l'autre en ce sens que :

$$
\mathcal{D} \circ \mathcal{D}^{\circ(-1)}=f^{\circ n} \text { pour un } n \in \mathbb{Z},
$$

l'entier $n$ dépendant des déterminations choisies pour définir la composition, et :

$$
\mathcal{D}^{\circ(-1)} \circ \mathcal{D}=g^{\circ n} \quad \text { pour un } n \in \mathbb{Z} .
$$


En passant aux revêtements universels des deux transversales, les identités précédentes nous disent que l'application de Dulac conjugue :

l'holonomie $\widetilde{g}: \widetilde{x} \mapsto \widetilde{x}+\frac{1}{\alpha}+\cdots \quad$ à la translation $\Delta_{y}^{\circ(-1)}: \widetilde{y} \mapsto \widetilde{y}-1$

et la translation $\Delta_{x}: \widetilde{x} \mapsto \widetilde{x}+1$ à l'holonomie $\tilde{f}^{\circ(-1)}: \widetilde{y} \mapsto \widetilde{y}-\alpha+\cdots$

En reprenant les estimations établies lors de la preuve du théorème de Mattei-Moussu 6.3, on montre que l'application de Dulac $\mathcal{D}$ est tangente à l'application $x \mapsto x^{-\alpha}$ à l'origine.

Remarque 8.1. Contrairement à ce que pourrait suggérer une lecture trop rapide du Théorème de Mattei-Moussu 6.3, un col n'est pas caractérisé par l'holonomie $f(z)=a z+\cdots$ d'une de ses séparatrices. En effet, l'holonomie détermine le col au choix près d'un logarithme $\alpha \in \mathbb{R}_{<0}$ de $a \in \mathbb{S}^{1}$. Par contre, l'application de Dulac, définie à composition à droite et à gauche près par des difféomorphismes $\varphi, \psi \in \operatorname{Diff}(\mathbb{C}, 0)$, caractérise le col. Tout d'abord, sa monodromie détermine l'holonomie et donc le col modulo un choix du logarithme. Maintenant, le choix du logarithme est donné ou bien par le comportement radial de $\mathcal{D}$ qui asymptotiquement est tangent à $x \mapsto$ $x^{-\alpha}$, ou bien par l'indice de l'image d'un lacet par $\mathcal{D}$. Attention, l'image d'un lacet d'indice 1 est un chemin $\gamma$ qui joint $\gamma(0)$ à $\gamma(1)=f^{\circ(-1)}(\gamma(0))$; cependant, il y a plusieurs manières non homotopiquement équivalentes de joindre ces deux points (voir discussion de la section 7.2). La partie réelle $-\operatorname{Ré}(\alpha)>0$ est donnée par la limite (bien définie) de la variation de l'argument de $\gamma$ lorsque le lacet de départ devient proche de l'origine.

Remarque 8.2. Chaque application de Dulac met en correspondance deux éléments $f, g \in \operatorname{Diff}(\mathbb{C}, 0)$ qui sont les deux holonomies d'un même col. Cette correspondance peut se construire de manière intrinsèque, sans utiliser les feuilletages, comme suit. Considérons un élément $g(z)=a z+\cdots \in$ $\operatorname{Diff}(\mathbb{C}, 0)$ avec $a \in \mathbb{S}^{1} ;$ quitte à le conjuguer par une homothétie, on peut supposer $g$ défini sur le disque $\mathbb{D}$. On relève sa dynamique sur le revêtement universel

$$
\mathbb{H} \rightarrow \mathbb{D}^{*} ; \widetilde{z} \mapsto \exp (2 i \pi \widetilde{z})
$$

en une dynamique $\widetilde{g}$; par construction, $\widetilde{g}$ commute à la transformation de revêtement $\Delta: \widetilde{z} \mapsto \widetilde{z}+1$. Bien sûr, le relèvement $\widetilde{g}$ n'est bien défini qu'à composition près par une itérée de $\Delta$, ce qui sous-entend que l'on a choisi un logarithme $\alpha$ de a. Maintenant, en recollant la demi-droite $i \mathbb{R}^{+}$ et son image par $\widetilde{g}$, on construit une surface de Riemann sur laquelle la dynamique quotient $\bar{\Delta}$ induite par la translation $\Delta$ est bien définie puisque $\widetilde{g}$ et $\Delta$ commutent. En reconnaissant cette surface comme étant le disque épointé $\mathbb{D}^{*}$ via le théorème d'uniformisation, on récupère la dynamique 
$f^{\circ}(-1)=\bar{\Delta}$. L'application de Dulac se déduit en relevant la projection par l'exponentielle.

Remarque 8.3. On montre assez facilement qu'une transformation multiforme $\mathbb{D}^{*} \rightarrow \mathbb{D}^{*}$, disons définie sur un nombre (fini) suffisamment grand de feuillets consécutifs de la surface de Riemann du logarithme, satisfaisant :

$$
\mathcal{D}\left(e^{2 i \pi} x\right)=f^{\circ(-1)} \circ \mathcal{D}(x)
$$

où $f(x)=a x+\cdots, a \in \mathbb{S}^{1}$, est l'application de Dulac d'un col.

Remarque 8.4. Étant donnée une dynamique sur $T_{x}$ induite par un élément $h \in \operatorname{Diff}(\mathbb{C}, 0)$, on peut considérer la dynamique $\mathcal{D}_{*} h=\mathcal{D} \circ h \circ \mathcal{D}^{\circ}(-1)$ induite sur $T_{y}$ en poussant par $\mathcal{D}$, ayant préalablement choisi des déterminations de $\mathcal{D}$ et de $\mathcal{D}^{\circ(-1)}$. Alors la dynamique $\mathcal{D}_{*} h$ est bien définie, i.e. sans monodromie, si, et seulement si, $h$ commute $\grave{a} g ;$ dans ce cas, chaque détermination de $\mathcal{D}_{*} h$ est un élément de $\operatorname{Diff}(\mathbb{C}, 0)$ qui commute à $f$ (en fait, on passe d'une détermination à une autre en composant par une itérée de $f$ ).

Remarque 8.5. Si $\mathcal{D}_{1}$ et $\mathcal{D}_{2}$ sont deux applications de Dulac, alors le composé $\mathcal{D}_{2} \circ \mathcal{D}_{1}$, des déterminations étant choisies, est encore l'application de Dulac d'un col si, et seulement si, l'holonomie au but $f_{1}$ de $\mathcal{D}_{1}$ (i.e. sa monodromie) commute à l'holonomie à la source $g_{2}$ de $\mathcal{D}_{2}$ (i.e. la monodromie de $\left.\mathcal{D}_{2}^{\circ(-1)}\right)$. En effet, la monodromie de $\mathcal{D}_{2} \circ \mathcal{D}_{1}$ est donnée par

$\mathcal{D}_{2} \circ \mathcal{D}_{1}\left(e^{2 i \pi} x\right)=\mathcal{D}_{2} \circ f_{1}^{\circ(-1)} \circ \mathcal{D}_{1}(x)=\left(\mathcal{D}_{2} \circ f_{1}^{\circ(-1)} \circ \mathcal{D}_{2}^{\circ(-1)}\right) \circ \mathcal{D}_{2} \circ \mathcal{D}_{1}(x)$ et on applique la remarque précédente.

\subsubsection{Type $(\operatorname{Lin})_{\text {col }}^{\mathrm{ex}}: \omega=x d y-\alpha y d x+\cdots$ non linéari- sable avec $\alpha \in \mathbb{R}_{<0} \backslash \mathbb{Q}_{<0}$}

La construction de la remarque 8.2, qui est très naturelle lorsque l'on considère l'application de Dulac d'un col, a été faite quelques années plus tôt par Étienne Ghys lorsqu'il a montré que l'ensemble :

$$
\mathcal{B}:=\left\{\alpha \in \mathbb{R} ; \exists f(z)=e^{2 i \pi \alpha} z+\cdots \in \operatorname{Diff}(\mathbb{C}, 0) \text { non linéarisable }\right\}
$$

était invariant par $\operatorname{PSL}(2, \mathbb{Z})$. En effet, $f$ est linéarisable si, et seulement si, $g$ est linéarisable (ici, nous le voyons comme corollaire du théorème de Mattei-Moussu), ce qui montre que $\mathcal{B}$ est invariant par $\alpha \mapsto \frac{1}{\alpha}$. Par ailleurs, $f$ est linéarisable si, et seulement si, $f^{\circ(-1)}$ est linéarisable et $\mathcal{B}$ est invariant par $\alpha \mapsto-\alpha$. Enfin, puisque $\alpha$ est défini par $f$ à addition près d'un entier, on obtient l'invariance de $\mathcal{B}$ par $\alpha \mapsto \alpha+1$ et donc par $\operatorname{PSL}(2, \mathbb{Z})$ en remarquant que ce dernier est engendré par $\alpha \mapsto \alpha+1$ et $\alpha \mapsto-\frac{1}{\alpha}$. Ceci justifie l'approche de la définition de $\mathcal{B}$ par le développement en fractions continues. 


\subsubsection{Type $(\text { Eucl })_{\text {col }}$ et $(\text { Eucl })_{\text {col }}^{\text {ex }}: \omega=p y d x+q x d y+\cdots$ non linéarisable}

Lorsque $\omega=\omega_{p / q, k, 0}$, c'est-à-dire dans la situation $(\text { Eucl })_{\text {col }}$ avec $\lambda=0$, l'intégrale première explicite nous donne :

$$
\mathcal{D}_{p / q, k, 0}(x)=x^{p / q} /\left(1+x^{k p} \log \left(x^{k p}\right)\right)^{1 / k q}
$$

Pour $p=q=1$, on retrouve exactement toutes les applications (voir section 3.4) :

$$
\varphi_{k, \lambda}=\psi_{k, 0}^{\circ(-1)} \circ \psi_{k, \lambda}: z \mapsto z /\left(1-\frac{\lambda}{2 i \pi} z^{k} \log \left(z^{k}\right)\right)^{1 / k}
$$

qui conjuguent la dynamique de $\exp \left(X_{k, \lambda}\right)$ à la dynamique $\exp \left(X_{k, 0}\right)$. En effet, $\varphi_{k, 2 i \pi}=\mathcal{D}_{1, k, 0}$ et on récupère $\varphi_{k, \lambda}$ en conjugant l'application de Dulac $\mathcal{D}_{1, k, 0}$ par une homothétie convenable puis en la composant au but par une itérée complexe convenable de $\exp \left(X_{k, 0}\right)$. Profitons-en pour remarquer que lorsque $\lambda=0$, c'est-à-dire lorsque l'invariant formel de $f$ est nul, alors celui de $g$ est nécessairement non nul. Ceci vaut aussi pour les types $(\text { Eucl })_{\mathrm{col}}^{\mathrm{ex}}$.

La correspondance de Dulac peut se voir comme le relèvement de la projection $T_{x} \rightarrow\left(T_{x} / g\right)$ par la projection $T_{y} \rightarrow\left(T_{y} / f\right)$. En effet, ces deux projections sont les passages au quotient par le feuilletage : les chapelets de sphères géométriques de $f$ et de $g$ sont les mêmes (bien que le nombre de pétales soit différent). Ceci nous donne les expressions sectorielles :

$$
\mathcal{D}=\psi_{f}^{\circ(-1)} \circ \psi_{g}
$$

où $\psi_{f}$ et $\psi_{g}$ sont des coordonnées de Leau respectives pour $f$ et $g$. Ceci n'a de sens que si l'on prend soin de remarquer préalablement qu'un pétale attractif pour $f$ correspond par $\mathcal{D}$ à un pétale répulsif pour $g$, et de choisir les coordonnées $\psi_{f}$ et $\psi_{g}$ associées à ces pétales.

La construction de la remarque 8.2 est celle que nous venons de décrire. Aussi, à chaque fois que deux automorphismes résonants $f, g \in \operatorname{Diff}(\mathbb{C}, 0)$ (non périodiques) ont même chapelet orienté mais à polarité inverse, alors ce sont les deux holonomies d'un même col résonant.

\subsubsection{Type nœud-col : $\omega=y d x+\cdots(\alpha=0)$}

Ici, nous ne considérons que des nœuds-cols avec deux séparatrices convergentes et nous allons considérer l'application de Dulac $\mathcal{D}$ allant d'une transversale $T_{x}$ à la variété forte $\{x=0\}$ vers une transversale $T_{y}$ à la variété centrale $\{y=0\}$. En reprenant les notations de la section 6.4, au-dessus de chaque pétale de $f$, le nœud-col est équivalent au feuilletage défini par $\omega_{p, \lambda}$ dont l'application de Dulac :

$$
\mathcal{D}_{p, \lambda}: x \mapsto x^{-\lambda} e^{1 / p x^{p}}
$$


est donnée par l'intégrale première $H_{p, \lambda}(x, y)=y x^{-\lambda} e^{1 / p x^{p}}$. On en déduit aisément que l'application $\mathcal{D}$ n'est définie que sur les ouverts $V_{k}^{+} \cap V_{k}^{-}$, $k=0, \ldots, p-1$, qu'elle envoie surjectivement sur $T_{y}$ (c'est même un revêtement). En ce sens, l'application de Dulac semi-disconnecte le feuilletage : toutes les feuilles coupant $T_{y}$ viennent recouper $T_{x}$; par contre, une partie des feuilles passant par $T_{x}$ vont se perdre dans "la partie nœud" du nœud-col et ne reviennent jamais couper $T_{y}$. On a une dissymétrie.

Il est remarquable aussi que l'application de Dulac coïncide avec les intégrales premières sectorielles de l'holonomie sur $T_{x}$ (voir section 3.4). En particulier, l'application de Dulac conjugue l'holonomie $f$ sur $T_{x}$ à l'indentité sur $T_{y}$.

\subsection{Les générateurs du pseudo-groupe d'holo- nomie}

Le pseudo-groupe d'holonomie d'une singularité de feuilletage $\mathcal{F}$ est à peu de choses près celui du feuilletage $\widetilde{\mathcal{F}}$ obtenu après réduction de la singularité : celui de $\widetilde{\mathcal{F}}$ contient en plus les points (en nombre fini!) correspondant aux composantes invariantes du diviseur exceptionnel. Nous commençons par décrire le pseudo-groupe des singularités réduites.

\subsubsection{Type hyperbolique ou col : $\omega=x d y-\alpha y d x+\cdots$ avec $\alpha \in \mathbb{C} \backslash \mathbb{R}_{\geq 0}$}

Une transversale complète au feuilletage (régulier) est donnée par la réunion disjointe de transversales $T_{x}$ et $T_{y}$ respectives aux courbes invariantes $\{x=0\}$ et $\{y=0\}$. Le pseudo-groupe $(T, G)$ est alors engendré par les applications d'holonomie respectives $f: T_{y} \rightarrow T_{y}$ et $g: T_{x} \rightarrow T_{x}$ et l'application de Dulac $\mathcal{D}: T_{x} \rightarrow T_{y}$ dont il suffit de donner une détermination $\underline{\mathcal{D}}$ puisque la relation $\mathcal{D}\left(e^{2 i \pi} x\right)=f^{\circ(-1)} \circ \mathcal{D}(x)$ permet de retrouver toutes les autres.

Il est quelque fois utile (et plus simple) de considérer les pseudo-groupes d'holonomie dans le complémentaire d'une ou des deux courbes invariantes. Par exemple, dans le complémentaire de $\{x=0\}$, une transversale complète est donnée par $T_{y}$ et le pseudo-groupe y est engendré par $f$. Maintenant, notons $\left(T^{*}, G\right)$ le pseudo-groupe du feuilletage restreint au complémentaire de $\{x y=0\}$.

Dans le cas hyperbolique $\alpha \notin \mathbb{R},\left(T^{*}, G\right)$ est équivalent au tore de réseau $\mathbb{Z}+\alpha \mathbb{Z}$.

Dans le cas $\alpha=-\frac{p}{q} \in \mathbb{Q}<0$, le feuilletage linéaire $p y d x+q x d y$ admet l'intégrale première $H(x, y)=x^{p} y^{q}$ et $\left(T^{*}, G\right)$ est équivalent à un disque épointé. Attention, le quotient de $T_{y}$ par $f$ n'est pas équivalent au disque en tant que pseudo-groupe mais doit être considéré comme un quotient 
orbifold; aussi le quotient $T / G$ possède deux points infiniment proches (non séparés), à savoir les deux courbes invariantes, et ces points sont tous deux orbifoldes de degré $p$ et $q$.

Dans le cas d'un feuilletage résonant $p y d x+q x d y+\cdots$, le pseudo-groupe $\left(T^{*}, G\right)$ est équivalent au chapelet de sphères (géométrique) correspondant indifféremment à $f$ ou à $g$.

\subsubsection{Type noud : $\omega=x d y-\alpha y d x$ avec $\alpha \in \mathbb{R}_{\geq 0} \backslash \mathbb{Q}_{\geq 0}$}

Ici, deux transversales $T_{x}$ et $T_{y}$ ne suffisent pas à définir une transversale complète au feuilletage, à moins de les choisir suffisamment grandes et proches de la singularité, ce que nous voulons éviter pour la suite. En travaillant avec l'intégrale première $H(x, y)=x^{-\alpha} y$, on vérifie facilement que le pseudo-groupe, dans le complément de la courbe invariante horizontale $\{y=0\}$, est équivalent au pseudo-groupe engendré sur $U_{x}:=\mathbb{C}$ par la rotation irrationnelle $g(x)=e^{2 i \pi / \alpha} x$ : c'est clair si l'on considère le feuilletage dans $\mathbb{C} \times \mathbb{C}^{*}$ et la transversale complète $\{y=1\}$; au voisinage de 0 , on obtient la même transversale en recollant les différentes transversales $T_{x}^{n}=\{y=1 / n,|x|<r\}$ à l'aide de l'holonomie radiale (une homothétie réelle). En particulier, $T_{x}$ est un voisinage de 0 dans $U_{x}$. Dans le complément de la droite verticale $\{y=0\}$, le pseudo-groupe est engendré sur $U_{y}:=\mathbb{C}$ par la rotation irrationnelle $f(y)=e^{2 i \pi \alpha} y$. Le pseudo-groupe $(T, G)$ se déduit en rajoutant l'application de Dulac

$$
\mathcal{D}: \mathbb{C}^{*} \rightarrow \mathbb{C}^{*} ; x \mapsto x^{-\alpha} .
$$

Le pseudo-groupe $\left(T^{*}, G\right)$, dans le complément de $\{x y=0\}$ est équivalent à

$$
\left(T^{*}, G\right) \sim\left(\mathbb{C}^{*},<e^{2 i \pi \alpha} z>\right) .
$$

Exemple 8.6. Le pseudo-groupe $(T, G)$ d'un nœud linéaire résonant

$$
\omega=p x d y-q y d x, \quad \frac{p}{q} \in \mathbb{Q}_{>0}
$$

est $\overline{\mathbb{C}}$ avec deux points orbifoldes (fermés) de degrés p et $q$. On réalise ainsi toutes les orbifoldes non uniformisables.

\subsubsection{Singularités réduites de type noud-col : $\omega=$ $y d x+\cdots(\alpha=0)$}

Le pseudo-groupe du complément à la variété forte se réduit à l'espace des feuilles tel qu'il est décrit dans la section 6.4.1. Notons que la donnée d'une transversale $T_{y}$ à la variété centrale, lorsque celle-ci est analytique, n'est jamais complète. Le pseudo-groupe $(T, G)$ se déduit de l'espace des 
feuilles en rajoutant une transversale $T_{x}$ à la variété forte ainsi que l'holonomie $f$ correspondante et l'application de Dulac telle qu'elle est décrite dans la section 8.1.7. La transversale $T_{x}$ est complète : elle intersecte toutes les feuilles excepté la variété centrale, lorsque celle-ci est analytique.

\subsubsection{Pseudo-groupe d'une singularité générale}

Considérons la réduction $\widetilde{\mathcal{F}}$ d'une singularité de feuilletage générale $\mathcal{F}$ et décrivons, à partir des données listées dans le Théorème 7.20, le pseudogroupe d'holonomie de $\widetilde{\mathcal{F}}$. Les notations sont celles de la fin de la section 7.4 .

Une transversale complète $T$ au feuilletage $\widetilde{\mathcal{F}}$ est donnée par la réunion de :

- les composantes $D_{i}$ du diviseur exceptionnel $D$ transverses à $\widetilde{\mathcal{F}}(i \in$ $\left.I^{\operatorname{tran}}\right)$,

- les transversales $T_{i}$ aux composantes $D_{i}$ de $D$ invariantes par $\widetilde{\mathcal{F}}$ $\left(i \in I^{\text {inv }}\right)$,

- les transversales supplémentaires nécessaires à la complétude au voisinage de chaque singularité de type nœud ou nœud-col apparaissant le long de $D$.

Il faudrait, afin d'être plus rigoureux, rajouter une transversale à chaque courbe invariante transverse à $D$. Nous ne le ferons pas afin de ne pas alourdir les notations dans la mesure où on ne s'intéresse ensuite qu'à un "ouvert de Zariski" du pseudo-groupe, c'est-à-dire à l'espace des feuilles modulo un nombre fini d'entre elles. Le pseudo-groupe d'holonomie du feuilletage est alors engendré sur $T$ par

- les générateurs d'holonomie projective $\varphi_{k}^{i}: T_{i} \rightarrow T_{i}$ pour chaque composante $D_{i}$ invariante par $\widetilde{\mathcal{F}}$,

- les applications de Dulac $\mathcal{D}_{i, j}: T_{i} \rightarrow T_{j}$ à chaque intersection $D_{i, j}$ de composantes invariantes par $\widetilde{\mathcal{F}}$,

- les identifications $\varphi_{i, j}: T_{i} \rightarrow D_{j}$ pour chaque intersection entre une composante invariante $D_{i}$ et une composante transverse $D_{j}$ de la transversale $T_{i}$ à $D_{i}$ avec un ouvert de $D_{j}$,

- le pseudo-groupe de chaque singularité $p_{i, j}$ ou $p_{k}^{i}$ lorsque cette singularité est de type nœud ou nœud-col.

Le pseudo-groupe $(T, G)$ ainsi défini est un objet difficile à décrire plus en profondeur en toute généralité. Nous terminons cette section par une classe de singularités pour lesquelles le pseudo-groupe admet a posteriori une description bien plus simple.

Commençons par la :

Proposition 8.7. Si $\mathcal{F}$ est non dicritique et si les singularités sont toutes de type col ou hyperbolique après résolution, alors toute transversale $T_{i}$ au 
diviseur $D$ reste complète au sens précédent : elle coupe toutes les feuilles sauf un nombre fini (à savoir la courbe invariante).

Démonstration. Il résulte de l'étude des singularités de type col ou hyperbolique que l'application de Dulac est localement surjective. Ainsi, toute feuille au voisinage de $D_{j}$ intersecte la transversale $T_{j}$, puis intersecte, via l'application de Dulac $\mathcal{D}_{j, k}: T_{j} \rightarrow T_{k}$ à chaque intersection $D_{j} \cap D_{k}$, la transversale $T_{k}$ de chaque composante voisine $D_{k}$. On conclue en raisonnant de proche en proche le long du diviseur.

La proposition reste vraie si l'on remplace $T_{i}$ par n'importe quelle germe de courbe $T$ à l'origine de $\mathbb{C}^{2}$ qui n'est pas invariante par le feuilletage $\mathcal{F}$. En effet, on se ramène à la preuve précédente quitte à éclater plus (pour désingulariser $T$ et sa position relative à $\mathcal{F}$ ) : on ne rajoute que des singularités de type col.

La proposition reste encore vraie si l'on autorise les nœud-cols sur la partie lisse du diviseur tels que la variété forte soit contenue dans le diviseur.

Exercice 8.8. Montrer qu'en présence d'un nœud, d'un nœud-col mal positionné (dans un coin ou dont la variété forte n'est pas contenue dans le diviseur) ou d'une composante dicritique dans la résolution, alors il existe une transversale $T_{i}$ qui n'est pas complète au sens précédent (intersectant toute feuille sauf un nombre fini, à savoir la courbe invariante).

Théorème 8.9. Supposons que $\mathcal{F}$ soit non dicritique et considérons le pseudo-groupe $\left(T^{*}, G\right)$ du complémentaire à la courbe invariante $C$. Si toutes les singularités apparaissant après réduction sont

- de type (Fin) col (avec intégrale première holomorphe) aux coins $D_{i} \cap$ $D_{j}$,

- de type col ou hyperbolique $\left(\alpha \in \mathbb{C} \backslash \mathbb{R}_{\geq 0}\right)$ sur la partie lisse de $D$, alors toute transversale $T$ à $D$ (sur la partie lisse) est complète et le pseudogroupe $G$ est induit sur un revêtement ramifié fini $\widetilde{T} \rightarrow T$ par un sousgroupe de $\operatorname{Diff}(\mathbb{C}, 0)$.

Démonstration. Pour chaque diviseur $D_{i}$ (par hypothèse invariant par $\widetilde{\mathcal{F}}$ ), notons $\widetilde{\mathcal{F}}_{i}$ le feuilletage restreint au voisinage tubulaire de $D_{i}$ et $\widetilde{\mathcal{F}}_{i}^{*}$, ce dernier feuilletage restreint au complémentaire de $D_{i}$ et des courbes invariantes transverses. Puisque toute singularité le long de $D_{i}$ est du type col ou hyperbolique, toute transversale $T_{i}$ est complète pour $\widetilde{\mathcal{F}}_{i}^{*}$. Le pseudogroupe de $\widetilde{\mathcal{F}}_{i}^{*}$ sur $T_{i}$ coïncide avec le pseudo-groupe d'holonomie projective $G_{i}$ défini dans la section 7.3, c'est-à-dire induit par le pseudo-groupe d'holonomie $G_{i} \subset \operatorname{Diff}(\mathbb{C}, 0)$ de la feuille sous-jacente $D_{i}^{*}=D_{i} \backslash \operatorname{Sing}\left(\mathcal{F}_{i}\right)$. Ceci résulte notamment du fait que, pour ce type de singularités, le pseudogroupe induit sur une transversale à la courbe invariante est engendré par 
l'holonomie. En un point d'intersection $p_{i, j}=D_{i} \cap D_{j}$, le feuilletage $\widetilde{\mathcal{F}}$ admet une intégrale première holomorphe $H_{i, j}$ localement conjuguée à $x^{p} y^{q}$. En la prolongeant le long des chemins $\delta_{i, j}$ et $\delta_{j, i}$ jusqu'aux transversales $T_{i}$ et $T_{j}$ (on reprend ici les notations du théorème 7.20 ), on observe que la restriction de $H_{i, j}$ aux transversales est de la forme

$$
H_{i, j}\left(z_{i}\right)=\left(f_{i}\left(z_{i}\right)\right)^{q} \quad \text { et } \quad H_{i, j}\left(z_{j}\right)=\left(f_{j}\left(z_{j}\right)\right)^{p} \quad \text { avec } \quad f_{i}, f_{j} \in \operatorname{Diff}(\mathbb{C}, 0)
$$

Nous allons comparer les dynamiques de $G_{i}$ et $G_{j}$ dans la variable $z=$ $\left(H_{i, j}\right)^{\frac{1}{p q}}$; cette variable est multiforme sur chacune des transversales, mais est bien définie sur un revêtement ramifié fini de chacune d'entre-elles. Par exemple, en relevant la variable $z$ par

$$
\pi_{i}: \widetilde{T}_{i} \rightarrow T_{i} ; \tilde{z}_{i} \mapsto\left(\tilde{z}_{i}\right)^{p}
$$

il vient $z=\tilde{f}_{i}\left(\tilde{z}_{i}\right)$ avec $\tilde{f}_{i} \in \operatorname{Diff}(\mathbb{C}, 0)$. La dynamique de $G_{i}$ se relève via $\pi_{i}$ en une dynamique sur $\widetilde{T}_{i}$ encore induite par un sous-groupe $\widetilde{G}_{i} \subset \operatorname{Diff}(\mathbb{C}, 0)$; ce relevé de $G_{i}$ contient notamment la transformation de revêtement $z \mapsto e^{\frac{2 i \pi}{p}} z$ : c'est une extension centrale de $G_{i}$ par $\mathbb{Z} / p \mathbb{Z}$. Maintenant, en passant à la coordonnée commune $z$ construite avec l'intégrale première $H_{i, j}$, on déduit que le pseudo-groupe du feuilletage au voisinage de $D_{i} \cup D_{j}$ est induit sur $\widetilde{T}_{i} \simeq_{z} \widetilde{T}_{j}$ par le sous-groupe $G_{i, j} \subset \operatorname{Diff}(\mathbb{C}, 0)$ engendré par $\widetilde{G}_{i}$ et $\widetilde{G}_{j}$ dans la variable $z$. Notons que $G_{i, j}$ est se relève encore en un sous-groupe de $\operatorname{Diff}(\mathbb{C}, 0)$ sur n'importe quel revêtement fini de la variable $z$; par suite, on peut recommencer avec une troisième composante $D_{k}$ intersectant $D_{i} \cup D_{j}$. De proche en proche, on montre ainsi l'existence, pour tout $D_{i}$, d'un entier $N_{i} \in \mathbb{N}^{*}$ tel que le pseudo-groupe global $G$ est bien défini comme sous-groupe de $\operatorname{Diff}(\mathbb{C}, 0)$ sur le revêtement à $N_{i}$ feuillets de $T_{i}$.

\subsection{Holonomie et intégrales premières}

Commençons par rappeler le résultat fondateur.

\subsubsection{Le Théorème de Mattei-Moussu}

Nous obtenons, comme corollaire du Théorème 8.9 , le théorème principal de [140] (voir aussi [148] pour une preuve plus élémentaire)

Théorème 8.10 (Mattei-Moussu). Soit $\mathcal{F}$ une singularité de feuilletage dont toutes les feuilles sont fermées dans un voisinage épointé $U^{*}$ de 0 et seules un nombre fini d'entre elles adhèrent à 0 dans le voisinage $U=U^{*} \cup\{0\}$. Alors $\mathcal{F}$ admet une intégrale première $H:\left(\mathbb{C}^{2}, 0\right) \rightarrow(\mathbb{C}, 0)$ holomorphe. De plus, on peut choisir $H$ à fibres connexes en dehors de 
la courbe invariante $C=H^{-1}(0)$, et toute autre intégrale première holomorphe se factorise par $H$.

Démonstration. Après résolution de $\mathcal{F}$, aucune composante $D_{i}$ ne peut être transverse au feuilletage : $\mathcal{F}$ est non dicritique. En effet, sinon, $\mathcal{F}$ aurait une infinité de courbes invariantes et par suite une infinité de feuilles adhérant à 0 . Par ailleurs, toutes les singularités réduites apparaissant après résolution doivent être de type (Fin) col car sinon, l'étude faite dans le Chapitre 6 montre qu'une infinité de feuilles de $\widetilde{\mathcal{F}}$ accumulerait ou bien des branches de la courbe invariante $C$, ou bien le diviseur; dans ce dernier cas, les feuilles correspondantes de $\mathcal{F}$ accumulent l'origine. Nous sommes en particulier dans la situation du Théorème 8.9 et le pseudo-groupe d'holonomie est donné par un sous-groupe $G \subset \operatorname{Diff}(\mathbb{C}, 0)$. D'après l'étude faite dans le Chapitre 4, $G$ doit être fini car sinon, une infinité d'orbites accumuleraient 0 et par suite une infinité de feuilles de $\widetilde{\mathcal{F}}$ accumuleraient $D$. Dans une coordonnée convenable $z$, le pseudo-groupe $G$ est donc linéaire, engendré disons par $f(z)=e^{2 i \pi / d} z$, et une intégrale première minimale est donnée par $H(z)=z^{d}$. Par construction, $H$ s'étend en une intégrale première holomorphe de $\widetilde{\mathcal{F}}$ dans le complémentaire de la courbe invariante; elle se prolonge par exemple par Riemann puisqu'elle est bornée. Toute autre intégrale première holomorphe de $\mathcal{F}$ doit être (en restriction à la transversale complète) invariante par l'holonomie $f$ et donc se factoriser à travers $H$.

Corollaire 8.11. Si une singularité $\mathcal{F}$ est topologiquement conjuguée à une autre singularité $\mathcal{F}^{\prime}$ admettant une intégrale première holomorphe, alors $\mathcal{F}$ admet aussi une intégrale première holomorphe.

Remarque 8.12. Une autre manière d'énoncer le Théorème 8.10 est de dire que l'existence d'une intégrale première holomorphe est caractérisée par le fait que l'espace des feuilles est un disque dont l'origine est la courbe invariante C. C'est en ces termes que le théorème est démontré dans [148].

Exemple 8.13. Pour qu'une singularité $\mathcal{F}$ admette une intégrale première holomorphe, il faut qu'elle soit non dicritique et qu'après réduction toutes les singularités réduites soient des cols holomorphes (de type (Fin) ${ }_{\mathrm{col}}$ ) et que tous les groupes d'holonomie projective soient finis. Ceci est nécessaire mais pas suffisant comme le montre l'exemple suivant. En utilisant le théorème 7.20, on peut construire un feuilletage $\mathcal{F}$ qui, après 2 éclatements, est réduit et satisfait :

- $D=D_{1} \cup D_{2}$ avec $D_{1} \cdot D_{1}=-2$ et $D_{2} \cdot D_{2}=-1$,

- au coin $D_{1} \cap D_{2}$, la singularité est un col de type $d(x y)=0$,

- les autres singularités sont linéarisables avec indice de Camacho-Sad $-\frac{1}{2}$ sur $D_{1}$ (il y en a 2 ) et $\pm \frac{1}{3}$ sur $D_{2}$ (il y en a 2 aussi),

- l'holonomie projective de $D_{1}$ et de $D_{2}$ est finie, engéndrée respectivement par $f\left(z_{1}\right)=-z_{1}$ et $g\left(z_{2}\right)=j z_{2} /\left(1-z_{2}\right)$, 
- l'application de Dulac identifie $z_{1}=z_{2}$.

Sous ces conditions, le pseudo-groupe d'holonomie est engendré dans la variable $z_{1}=z_{2}$ par $f$ et $g$ et n'est donc pas fini.

Depuis que Jean-François Mattei et Robert Moussu ont démontré le Théorème 8.10 en 1980, de nombreux travaux ont cherché à étendre ce résultat à des intégrales premières plus générales (méromorphes, multiformes de type Darboux, Liouville ou Riccati) en cherchant des critères d'existence portant sur l'holonomie. Citons notamment les articles [57], [115], [218], [161], [199], [61], [15], [220]. À l'inverse, dans [12] et [120], on trouve des critères topologiques de non existence de certaines intégrales premières. Dans [127], Bernard Malgrange propose une définition générale de groupoïde de Galois $\operatorname{Gal}(\mathcal{F})$ d'un feuilletage $\mathcal{F}$ (de dimension et codimension arbitraire sur une variété complexe) qui permit dans [48] de rassembler toutes les approches précédentes dans un énoncé d'une simplicité remarquable : le type d'intégrale première admise par le feuilletage dépend de la dimension transverse du groupoïde de Galois : 0, 1, 2 ou 3 respectivement. Pour une singularité très générale, la dimension est infinie et il n'y a pas d'intégrale première raisonnable. Le but des sections suivantes est de voir ce qui subsiste de cet énoncé dans un cadre topologique.

\subsubsection{Groupoïde de Galois et structure transverse au feuilletage}

Le groupoïde de Galois $\operatorname{Gal}(\mathcal{F})$ d'un feuilletage $\mathcal{F}$ est la clôture de Lie de son pseudo-groupe tangent (voir section 5.2) dans le sens que nous avons vaguement décrit dans la section 4.5. Le pseudo-groupe des transformations basiques étant lui même un pseudo-groupe de Lie au sens de Malgrange, il contient le groupoïde de Galois (qui lui-même contient le pseudo-groupe tangent). En un point générique du feuilletage, le groupoïde de Galois est le produit de sa restriction à une transversale $T$ avec le pseudo-groupe tangent local. Il va dégénérer le long d'un sous-ensemble analytique $Z$ invariant par $\mathcal{F}$, la réunion de quelques branches de la courbe invariante $Z \subset C$ dans notre situation. Sa restriction à une transversale complète $T$ va coïncider avec la clôture de Lie $\bar{G}^{\text {Lie }}$ du pseudo-groupe d'holonomie $G$. Si le groupoïde a l'avantage d'être intrinsèquement défini par le feuilletage, i.e. ne nécessite pas de choix préalable de transversale pour le définir, il est néanmoins pratique de le restreindre à une transversale complète, ne serait-ce que pour le décrire ou le calculer. Lorsque $\operatorname{Gal}(\mathcal{F})$ n'est pas le pseudo-groupe maximal de toutes les transformations basiques, alors sa dimension transverse, i.e. la dimension de $\bar{G}^{\text {Lie }}$ est $\leq 3$ : en un point générique, $\bar{G}^{\text {Lie }}$ est un des pseudo-groupes correspondant au Théorème de Lie; en un point de $Z \cap T$, c'est un des modèles singuliers décrit dans la section 4.5. 
Rappelons qu'un feuilletage régulier $\mathcal{F}$ (de codimension 1) sur une variété $M$ est transversalement euclidien, affine ou projectif lorsque l'on peut trouver un atlas de submersions $H_{i}: U_{i} \rightarrow \mathbb{C}$ le définissant tel que les applications de transition $H_{i}=\varphi_{i, j} \circ H_{j}$ soient toutes de la forme

$$
\varphi_{i, j}(z)=z+b_{i, j}, \quad a_{i, j} z+b_{i, j} \quad \text { ou } \quad \frac{a_{i, j} z+b_{i, j}}{c_{i, j} z+d_{i, j}}
$$

respectivement. En d'autres termes, il existe un système de coordonnées sur la tranversale complète $T$ à travers lesquelles le pseudo-groupe d'holonomie $G$ du feuilletage est un pseudo-groupe de translations, de transformations affines ou projectives. Dans ce cas, on peut construire une intégrale première "globale multiforme" $H: \widetilde{M} \rightarrow \mathbb{C}$ (ou $\overline{\mathbb{C}}$ dans le cas projectif) bien définie sur le revêtement universel $\widetilde{M}$ de $M$ et dont la monodromie est euclidienne, affine ou projective : pour tout élément $\gamma \in \pi_{1}(M)$, la nouvelle détermination $H^{\gamma}$ obtenu par prolongement de $H$ le long de $\gamma$ est donnée respectivement par

$$
H^{\gamma}=H+b_{\gamma}, \quad a_{\gamma} H+b_{\gamma} \quad \text { ou } \quad \frac{a_{\gamma} H+b_{\gamma}}{c_{\gamma} H+d_{\gamma}}
$$

pour des constantes $a_{\gamma}, b_{\gamma}, c_{\gamma}, d_{\gamma}$ convenables. Cette intégrale première est unique modulo composition au but par une transformation du groupe corespondant : c'est la développante de la structure (voir [85]).

Si l'on revient au cas singulier qui nous intéresse ici, lorsque le groupoïde de Galois $\operatorname{Gal}(\mathcal{F})$ est de dimension transverse 1, 2 ou 3, le feuilletage $\mathcal{F}$ est respectivement transversalement euclidien, affine ou projectif en restriction au complémentaire de $Z$. La définition de pseudo-groupe de Lie impose une certaine modération à la dégénérescence du pseudo-groupe le long de $Z$ : il est défini par des équations différentielles méromorphes le long de $Z$ (voir section 4.5). Quelques années plus tôt, Bruno Scardua donnait une définition de feuilletage singulier transversalement euclidien, affine ou projectif dans [199] avec pôles le long d'un diviseur $Z$ qui a posteriori coïncide avec celle de Malgrange, ce qui rend très naturelles ces définitions. Il est important de remarquer ici qu'un feuilletage transversalement projectif $\mathcal{F}$ sur le voisinage épointé de $0 \in \mathbb{C}^{2}$ possède nécessairement une intégrale première méromorphe, ce qui le rend transversalement trivial! En effet, l'application développante $H$ introduite plus haut nous fournit une intégrale première multiforme dans le complément de $Z$; si $Z$ était réduit à 0 , alors $H$ serait uniforme par simple connexité du voisinage épointé. Ainsi, dans le cas singulier local, il est naturel de demander à ce que la structure transverse dégénère au moins le long d'un ensemble de codimension 1 si l'on veut considérer des singularités plus intéressantes du point de vue dynamique. 


\subsubsection{Holonomie finie et intégrales premières méro- morphes}

Guy Casale montre dans [48] le

Théorème 8.14 (Casale). Le feuilletage $\mathcal{F}$ admet une intégrale première méromorphe si, et seulement si, le groupoïde de $\operatorname{Galois} \operatorname{Gal}(\mathcal{F})$ est de dimension transverse 0 .

Par contre, il n'y a pas de caractérisation topologique des singularités admettant une intégrale première méromorphe (autrement dit dont le groupoïde de Galois est de dimension transverse 0). Le premier contre-exemple est donné par Masakazu Suzuki dans [215] : il s'agit des deux feuilletages $\mathcal{F}_{0}$ et $\mathcal{F}_{1}$ définis respectivement par

$\omega_{0}=\left(2 y^{2}+x^{3}\right) d x-2 x y d y \quad$ et $\quad \omega_{1}=\left(y^{3}+y^{2}-x y\right) d x-\left(2 x y^{2}+x y-x^{2}\right) d y$;

admettant les intégrales premières respectives

$$
H_{0}=\frac{y^{2}-x^{3}}{x^{2}} \quad \text { et } \quad H_{1}=\frac{x}{y} e^{\frac{y(y+1)}{x}} .
$$

Suzuki montre que ces feuilletages sont topologiquement conjugués et que le second n'admet pas d'intégrale première méromorphe. Nous allons revenir sur ces exemples dans un instant.

Lemme 8.15. Soit $\mathcal{F}$ une singularité de feuilletage dont toutes les feuilles sont fermées dans un voisinage épointé $U^{*}$ de 0 . Alors le feuilletage réduit $\widetilde{\mathcal{F}}$ admet une intégrale première holomorphe $H$ au voisinage de tout sousarbre $D^{\prime} \subset D$ connexe et invariant par $\widetilde{\mathcal{F}}$. De plus, on peut choisir $H$ minimale (i.e. à fibre connexe) de sorte que son image s'identifie avec l'espace des feuilles au voisinage de $D^{\prime}$.

Ici et dans la suite, par sous-arbre, nous entendons que $D^{\prime}$ est une réunion connexe de composantes irréductibles de $D$.

Démonstration. Le théorème de Remmert-Stein implique que toutes les feuilles sont analytiques en 0 . Par suite, les feuilles de $\widetilde{\mathcal{F}}$ sont aussi analytiques et donc fermées, quitte à leur rajouter un nombre fini de points de $D$ : si une feuille accumule un ouvert de $D$, alors elle est contenue dans $D$. On peut alors reprendre les arguments de la preuve du théorème 8.10 le long d'un sous-arbre invariant $D^{\prime}$.

Le pseudo-groupe d'une singularité $\mathcal{F}$ à feuilles fermées (voir lemme 8.15) peut être décrit de la manière suivante. Notons :

- $D_{1}^{\text {dicr }}, \ldots, D_{k}^{\text {dicr }} \subset D$ les composantes dicritiques du diviseur exceptionnel $D$ (les composantes irréductibles transverses au feuilletage réduit $\widetilde{\mathcal{F}}$ ) ; 
- $D_{1}^{\text {inv }}, \ldots, D_{l}^{\text {inv }} \subset D$ les sous-arbres invariants maximaux (pour l'inclusion) ;

- $H_{j}$ une intégrale première minimale du feuilletage réduit $\left.\widetilde{\mathcal{F}}\right|_{V_{j}}$ au voisinage de $D_{j}^{\text {inv }}$ (voir lemme 8.15 ) pour $i=1, \ldots, l$.

Passons rapidement sur les deux cas suivants.

Remarque 8.16. Lorsque $k=0$, le diviseur $D$ est totalement invariant et $\mathcal{F}$ possède une intégrale première holomorphe. Lorsque $l=0$, le diviseur $D$ est réduit à une composante irréductible dicritique; le feuilletage $\mathcal{F}$ est alors radial, conjugué à $x d y-y d x=0$ : il admet l'intégrale première méromorphe $H(x, y)=\frac{y}{x}$ et son pseudo-groupe est $(T, G)=(\overline{\mathbb{C}},\{i d\})$.

Supposons désormais $k, l>0$. Une transversale complète au feuilletage $\mathcal{F}$ est donnée par la réunion $T=D_{1}^{\text {dicr }} \cup \cdots \cup D_{k}^{\text {dicr }}$, mais il est commode de décrire le pseudo-groupe à l'aide d'une transversale plus grosse. On rajoute, pour chaque sous-arbre invariant $D_{j}^{\text {inv }}$, l'espace des feuilles $T_{j}$ au voisinage, c'est-à-dire l'image de $H_{j}$. Le pseudo-groupe (global) de $\mathcal{F}$ est alors engendré sur $T^{\prime}=T \cup T_{1} \cup \cdots \cup T_{l}$ par les restrictions $H_{j} \mid T$ (à valeur dans $T_{j}$ ) pour $j=1, \ldots, l$ : autrement dit, on obtient, pour chaque intersection (non vide) $p_{i, j}=D_{i}^{\text {dicr }} \cap D_{j}^{\text {inv }}$, un germe de revêtement ramifié $\left.H_{j}\right|_{T}:\left(D_{i}^{\text {dicr }}, p_{i, j}\right) \rightarrow\left(T_{j}, 0\right)$ (où $T_{j} \subset \mathbb{C}$ est un voisinage ouvert de 0 ). Si l'on revient à la transversale $T=D_{1}^{\text {dicr }} \cup \cdots \cup D_{k}^{\text {dicr }}$, le pseudo-groupe $G$ induit par $\widetilde{\mathcal{F}}$ est engendré par les (germes de) correspondances $H_{j}^{\circ(-1)} \circ H_{j}$ là où ça a un sens, à savoir :

- le groupe fini d'invariance de $H_{j}$ à chaque point $p_{i, j}$,

- le germe de correspondance induit par $H_{j}$ entre chaque paire de points $p_{i_{1}, j}$ et $p_{i_{2}, j}$ (lorsque $D_{j}^{\text {inv }}$ intersecte plusieurs composantes dicritiques).

Le critère suivant est une variante du théorème A de [29].

Proposition 8.17. Soit $\mathcal{F}$ une singularité de feuilletage dont toutes les feuilles sont fermées dans un voisinage épointé $U^{*}$ de 0 . Avec les notations précédentes, le feuilletage $\mathcal{F}$ admet une intégrale première méromorphe si, et seulement si, son pseudo-groupe $(T, G)$ admet une fonction méromorphe, i.e. une fonction rationnelle $H: T \rightarrow \overline{\mathbb{C}}$ invariante par $G$.

Démonstration. Si $H$ est une intégrale première méromorphe de $\mathcal{F}$, alors sa restriction à $T$ est rationnelle et est par construction invariante par $G$ (qui identifie les points d'une même feuille). Réciproquement, si $H: T \rightarrow \overline{\mathbb{C}}$ est invariante par $G$, alors elle se factorise au voisinage de chaque $D_{j}^{\text {inv }}$ par l'intégrale première minimale, $H=\phi_{j} \circ H_{j}$, ce qui permet de l'étendre en un intégrale première méromorphe de $\widetilde{\mathcal{F}}$ au voisinage de $D_{j}^{\text {inv }}$; elle s'étend aussi en une intégrale première au voisinage de chaque composante dicritique $D_{i}^{\text {dicr }}$. 
Exemple 8.18. Dans les 2 exemples de Suzuki, $\mathcal{F}_{0}$ et $\mathcal{F}_{1}$ au-dessus, le feuilletage devient régulier après un éclatement, transverse au diviseur exceptionnel sauf en un point où il y a une tangence simple, à savoir les points respectifs $(x: y)=(1: 0)$ et $(1: 1)$; ce premier diviseur nous fournit une transversale complète $T=D_{1}$ pour le feuilletage. Le feuilletage réduit s'obtient après 2 éclatements supplémentaires; ces 2 nouvelles composantes de $D$ forment la partie invariante $D^{\mathrm{inv}}=D_{2} \cup D_{3}$ du feuilletage. Dans le cas de $\mathcal{F}_{0}$, notons que le cusp $\left\{y^{2}=x^{3}\right\}$ est une feuille du feuilletage: l'algorithme de réduction est le même que pour les singularités cuspidales de la section 7.1.2 bien que le feuilletage soit très différent. Pour ces deux exemples, $\mathcal{F}_{0}$ et $\mathcal{F}_{1}$, le pseudo-groupe du feuilletage est engendré sur $T$ par un germe d'involution, à savoir celle qui met en relation les deux points de la même feuille au voisinage du point de tangence avec le feuilletage. C'est aussi l'involution qui laisse invariante l'intégrale première locale du feuilletage qui a un point critique une fois restreinte au diviseur. En choisissant une coordonnée projective $t$ sur $T \simeq \overline{\mathbb{C}}$ pour laquelle le point de tangence est $t=0$, le pseudo-groupe est donné par

$$
(T, G)=(\overline{\mathbb{C}}, f) \quad \text { où } f(t)=-t+\cdots \text { et } f \circ f=\text { identité. }
$$

Ce type de singularités, "désingularisées" après un éclatement, ont été étudiées systématiquement dans [57, 99, 156, 26, 157]. Dans le cas le plus simple (une tangence simple après un éclatement), la donnée de l'involution $f$ modulo l'action naturelle de $\operatorname{PGL}(2, \mathbb{C})$ sur $T$ caractérise le feuilletage $\mathcal{F}$ modulo Diff $\left(\mathbb{C}^{2}, 0\right)$ (voir [99]). Par ailleurs, tout germe d'involution est réalisable par un tel feuilletage : on peut le voir comme une application du 7.20.

Pour le premier exemple $\mathcal{F}_{0}$, la restriction de l'intégrale première $\left.H_{0}\right|_{T}$ : $t \rightarrow t^{2}$ induit l'involution $f_{0}(t)=-t$ dans la coordonnée projective $t=y / x$. Pour le second exemple $\mathcal{F}_{1}$, l'intégrale première $H_{1}$, bien que transcendante, est holomorphe et régulière au point de tangence $t=1$ (toujours avec $t=y / x)$ après le premier éclatement. Le germe d'involution $f_{1}(t)$ est donc défini, en $t=1$, par le point critique de sa restriction $\left.H_{1}\right|_{T}: t \rightarrow \frac{e^{t}}{t}$. Or, on peut montrer (voir [122], exemple 6.4) que le prolongement analytique du germe d'involution $f_{1}(t)$ est multiforme avec une infinité de feuillets. Si $\mathcal{F}_{1}$ admettait une intégrale première méromorphe $H$, sa restriction (rationnelle) $\left.H\right|_{T}$ serait une intégrale première de $f_{1}$, ce qui forcerait $f_{1}$ à être le germe de la fonction algébrique définie par $H(f(t))=H(t)$; ceci contredit le fait que $f_{1}$ a une infinité de feuillets. Donc $\mathcal{F}_{1}$ n'admet pas d'intégrale première méromorphe. Cependant, notons que $\mathcal{F}_{1}$ est défini par la forme fermée

$$
\frac{\omega_{1}}{x^{2} y}=\frac{d x}{x}-\frac{d y}{y}+d\left(\frac{y(y+1)}{x}\right)=\frac{d H_{1}}{H_{1}}
$$

et est donc intégrable au sens de la prochaine section : son groupoïde de 
Galois est de dimension transverse 1. On peut aussi construire un exemple $\mathcal{F}_{2}$ du même type et dont le groupoïde de Galois est de dimension transverse infinie : il suffit de faire en sorte qu'il ne soit pas de dimension $\leq 3$, c'est$\grave{a}$-dire transversalement projectif au sens de la section 8.3.6. Pour cela, on choisira une involution dont le prolongement analytique maximal ne va pas au-delà d'un disque : ceci empêchera l'existence d'une développante pour la structure projective (voir section 8.3.6).

Pour montrer que $\mathcal{F}_{0}$ et $\mathcal{F}_{1}$ (ou plus généralement deux feuilletages quelconques de cette famille) sont topologiquement conjugués, il suffit de construire tout d'abord une conjugaison topologique des pseudo-groupes : les arguments sont similaires à ceux de la preuve du théorème 3.34. Ensuite, on relève cette conjugaison aux feuilletages à l'aide d'un feuilletage auxiliaire génériquement transverse (voir [99]).

Remarque 8.19. L'exemple précédent n'est pas anecdotique, mais illustre plutôt un fait général : une singularité de feuilletage $\mathcal{F}$ admettant une intégrale première méromophe est très souvent topologiquement conjuguée à une singularité $\mathcal{F}^{\prime}$ n'en admettant pas. Par exemple, il suffit que le pseudogroupe $(T, G)$ du feuilletage contienne un germe de difféomorphisme (nécessairement périodique) non trivial sur une des composantes $D_{i}^{\text {dicr }} d e T$. Par chirurgie, on peut modifier ce germe de difféomorphisme afin qu'il ne puisse plus se prolonger au-delà d'un disque donné. Il faut ensuite relever cette chirurgie au feuilletage et surtout démontrer la conjugaison topologique, ce qui est assez technique (voir [99]). Par ailleurs, dès que le feuilletage réduit possède deux composantes dicritiques distinctes $D_{1}^{\text {dicr }}$ et $D_{2}^{\text {dicr }}$, alors on peut encore, par chirurgie, modifier le germe de correspondance $H_{j}^{\circ(-1)} \circ H_{j}$ qui les relie de sorte que ce germe ne soit plus le germe d'une fonction algébrique globale $D_{1}^{\text {dicr }} \rightarrow D_{2}^{\text {dicr }}$ (par exemple, ne se prolonge pas analytiquement au-delà d'un disque donné).

$A$ contrario, si $\mathcal{F}$ n'a pas de composante dicritique, alors le théorème 8.10 nous dit que $\mathcal{F}$ (et donc tout feuilletage $\mathcal{F}^{\prime}$ topologiquement conjugué $\grave{a} \mathcal{F}$ ) aura une intégrale première holomorphe.

De même, on peut montrer que les nœuds rationnels définis par $H(x, y)=$ $y^{p} / x^{q}$ sont topologiquement rigides. En effet, supposons qu'une singularité $\mathcal{F}^{\prime}$ soit topologiquement conjuguée à $\mathcal{F}_{H}$. D'après le lemme 8.15, le feuilletage réduit associé à $\mathcal{F}^{\prime}$ ne possède pas de nœud-col : on peut alors appliquer les résultats de [37] et déduire que $\mathcal{F}^{\prime}$ a le même nombre de Milnor $\mu=1$ que $\mathcal{F}_{H}$. Ainsi $\mathcal{F}^{\prime}$ est non dégénérée et il n'est pas difficile de déduire que $\mathcal{F}^{\prime}$ ne peut être qu'un nœud rationnel, donc linéarisable. Par ailleurs, la réduction des singularités est la même (toujours d'après [37]) : il s'agit donc précisément du nœud défini par $H$.

L'analogue suivant du théorème 8.10 est démontré par Martine Klughertz dans [99] : 
Théorème 8.20 (Klughertz). Soit $\mathcal{F}$ une singularité de feuilletage dont toutes les feuilles sont fermées dans un voisinage épointé $U^{*}$ de 0 . Alors $\mathcal{F}$ est topologiquement conjugué à une singularité $\mathcal{F}^{\prime}$ admettant une intégrale première méromorphe.

Nous terminons la section par un problème toujours ouvert, posé dans [57] :

Conjecture 8.21. Tout germe de fonction méromorphe à l'origine de $\mathbb{C}^{2}$ est analytiquement conjugué au germe d'une fonction rationnelle $F \in$ $\mathbb{C}(x, y)$.

Un germe de fonction holomorphe dans $\left(\mathbb{C}^{2}, 0\right)$ est conjugué à un polynôme : c'est un cas particulier d'un résultat de Mather sur la détermination finie des germes de fonctions à singularité isolée dans $\left(\mathbb{C}^{n}, 0\right)$ (voir [57]). Un résultat partiel vers cette conjecture est donné dans [49] mais elle reste ouverte aujourd'hui.

\subsubsection{Holonomie abélienne et intégrales premières de type Darboux}

On dit qu'un germe de feuilletage $\mathcal{F}$ est Darboux intégrable lorsqu'il peut être défini par une 1-forme méromorphe $\omega$ fermée : $d \omega=0$. Dans ce cas, l'intégrale première

$$
H=\int \omega
$$

est à monodromie additive (euclidienne). Ce type de singularités à été étudié en détails dans [57]. On peut écrire

$$
\omega=\lambda_{1} \frac{d F_{1}}{F_{1}}+\cdots+\lambda_{n} \frac{d F_{n}}{F_{n}}+d G, \quad F_{i}, G \text { méromorphes, } \lambda_{i} \in \mathbb{C}^{*}
$$

ce qui nous donne en intégrant une intégrale première (multiforme) de type Darboux

$$
H=\lambda_{1} \log \left(F_{1}\right)+\cdots+\lambda_{n} \log \left(F_{n}\right)+G \text { ou encore } H=F_{1}^{\lambda_{1}} \cdots F_{n}^{\lambda_{n}} e^{G} ;
$$

la première étant à monodromie euclidienne, la seconde à monodromie linéaire. On dit que le feuilletage est transversalement euclidien dans ce cas.

Les singularités réduites transversalement euclidiennes sont les linéaires

$$
\alpha \frac{d x}{x}-\frac{d y}{y}, \quad \alpha \in \mathbb{C}^{*}
$$

et les modèles formels de Dulac pour les cols et noud-cols (voir chapitre $6)$

$$
\frac{d x}{x}-\frac{1+\lambda u^{k}}{u^{k+1}} d u, \quad \lambda \in \mathbb{C}, k \in \mathbb{N}^{*}
$$


où $u:=x^{p} y^{q}$ dans le cas d'un nœud de type $(p, q)$ et $u=y$ dans le cas d'un nœud-col.

Dans [161], Emmanuel Paul donne des conditions nécessaires et suffisantes pour l'existence d'intégrale première de type Darboux pour une singularité générale $\mathcal{F}$. Décrivons ces conditions dans le cadre suivant. Supposons que le feuilletage $\widetilde{F}$, après résolution, n'admet que des composantes $D_{i}$ invariantes, et n'admet pas de singularité de type nœud-col. Ce type de feuilletages est appelé "courbe généralisée non dicritique" dans [37]. Dans ce cas, décomposons $D=\cup_{j} D_{j}^{\text {hol }}$ où les $D_{j}^{\text {hol }}$ sont les composantes connexes maximales de $D$ (unions de composantes irréductibles $D_{i}$ ) pour lesquelles les coins sont à intégrale première holomorphe. À une telle composante, on peut associer un sous-groupe $G_{j} \subset \operatorname{Diff}(\mathbb{C}, 0)$ qui est le pseudo-groupe d'holonomie vu sur n'importe quelle transversale à revêtement fini près (voir preuve du Théorème 8.9).

Théorème 8.22 (Paul). Soit $\mathcal{F}$ une courbe généralisée non dicritique, et les $G_{j}$ comme au-dessus. Alors $\mathcal{F}$ est Darboux intégrable, i.e. peut être défini par une 1-forme méromorphe fermée $\omega$, si, et seulement si, tous les $G_{j}$ définis comme au-dessus sont abéliens, analytiquement linéarisables ou normalisables (type $\mathbb{L}$ ou $\mathbb{E}_{p, \lambda}$, voir section 4.1).

On peut vérifier que l'énoncé reste vrai en présence de nœud-cols sur la partie lisse de $D$ avec variété forte contenue dans $D$.

Démonstration. Supposons $\mathcal{F}$ de type Darboux. Après résolution des singularités, la 1-forme $\omega$ se relève en une 1-forme méromorphe $\widetilde{\omega}$ définissant le feuilletage $\widetilde{\mathcal{F}}$. Par construction, la restriction de $\widetilde{\omega}$ à une transversale $T_{j}$ à $D_{j}^{\text {hol }}$ est invariante par le pseudo-groupe d'holonomie $G_{j}$ correspondant. Quitte à passer à un revêtement fini $\widetilde{T}_{j} \rightarrow T_{j}$, on peut supposer $G_{j} \subset \operatorname{Diff}(\mathbb{C}, 0)$. D'après les Propositions 4.1 et 4.14 , le pseudo-groupe d'holonomie $G_{j}$ est du type $\mathbb{L}$ ou $\mathbb{E}_{p, \lambda}$ selon que la restriction de $\widetilde{\omega}$ à la transversale possède un pôle simple ou multiple. Notons que la dichotomie $\mathbb{L} / \mathbb{E}_{p, \lambda}$ (ou pôle simple / multiple pour $\widetilde{\omega}$ ) est stable par revêtement ramifié et ne dépend donc pas de la transversale $T_{j}$ choisie le long de $D_{j}^{\text {hol }}$, ni de sa ramification $\widetilde{T}_{j}$.

Réciproquement, supposons tous les $G_{j}$ est du type $\mathbb{L}$ ou $\mathbb{E}_{p, \lambda}$. En particulier, toutes les singularités réduites le long de $D_{j}^{\text {hol }}$, puis de procheen-proche le long de $D$, sont du type linéaire (Lin) (hyperbolique, col ou nœud, voir section 6.2) ou col résonant euclidien (Eucl) col (section 6.3.2). En effet, dans le cas des cols, puisque l'holonomie (contenue dans $G_{j}$ ) est linéarisable / normalisable, il en est de même pour les singularités de feuilletage.

À l'intersection de deux composantes $D_{j_{1}}^{\text {hol }} \cap D_{j_{2}}^{\text {hol }}$, le feuilletage n'admet pas d'intégrale première holomorphe, et son holonomie est donc nécessairement d'ordre infini, ce qui implique que $G_{j_{1}}$ et $G_{j_{2}}$ sont d'ordre infini. 
On en déduit que chaque groupe $G_{j}$ est infini, excepté dans le cas où tous les coins sont à intégrale première holomorphe, et donc $D_{j}^{\text {hol }}=D$ (une seule composante). Dans ce dernier cas, $G$ peut être fini, mais alors les feuilles sont fermées et le Théorème 8.10 (Mattei-Moussu) nous dit que $\mathcal{F}$ possède une intégrale première holomorphe $H$; on en déduit alors que $\mathcal{F}$ est défini par la 1-forme holomorphe fermée $\omega=d H$.

Supposons donc pour la suite que (le ou) les $G_{j}$ sont infinis. Ainsi, il existe (à multiple constant près) une unique 1-forme méromorphe $\omega_{j}$ invariante par $G_{j}$. Pour chaque singularité de feuilletage le long de $D_{j}^{\text {hol }}$, cette 1-forme invariante par l'holonomie s'étend de manière unique en une 1-forme méromorphe fermée définissant le feuilletage. De proche-en-proche, on peut étendre $\omega_{j}$ en une 1-forme méromorphe fermée définissant $\widetilde{F}$ au voisinage de $D_{j}^{\text {hol }}$. Lorsqu'il n'y a qu'une composante $D_{j}^{\text {hol }}=D$, c'est terminé puisque la 1-forme descend en une 1-forme méromorphe fermée définissant $F$ sur $(\mathbb{C}, 0)$. Si par contre il y a plusieurs composantes, à chaque intersection $D_{j_{1}}^{\text {hol }} \cap D_{j_{2}}^{\text {hol }}$ on a $\omega_{j_{1}}=c_{1,2} \omega_{j_{2}}$ pour une constante $c_{1,2} \in \mathbb{C}^{*}$. Puisque $D$ est un arbre (pas de topologie), on peut modifier les $\omega_{j}$ pour qu'ils se recollent, et on conclue comme avant.

Théorème 8.23 (Casale). Soit $\mathcal{F}=\mathcal{F}_{\omega}$ avec $\omega$ holomorphe. Le groupoïde de Galois $\operatorname{Gal}(\mathcal{F})$ est de dimension transverse $\leq 1$ si, et seulement si :

$$
d \omega=\frac{1}{k} \frac{d F}{F} \wedge \omega
$$

pour un entier $k \in \mathbb{Z}_{>0}$ et une fonction méromorphe $F$.

La condition entre $\omega$ et $F$ est équivalente à $d\left(\frac{\omega}{F^{1 / k}}\right)=0$. Autrement dit, sur un revêtement fini de degré $k$, feuilletage relevé est défini par une 1forme méromorphe fermée. Attention, le revêtement défini par $z^{k}=F(x, y)$ peut être singulier au-dessus de 0 et il faut éclater pour désingulariser la surface.

Exemple 8.24. Illustrons ceci sur l'exemple des singularités cuspidales (voir section 7.1.2). Dans ce cas, $D=D_{1} \cup D_{2} \cup D_{3}$ est à coins holomorphes et le pseudo-groupe $G$ du feuilletage s'identifie au groupe d'holonomie projective $\left\langle f_{1}, f_{2}\right\rangle$ (voir exemple 7.16). Dans l'exemple étudié dans [147], ce groupe est explicitement donné par

$$
\langle z \mapsto-z, z \mapsto j z /(1-z)\rangle, \quad j=e^{\frac{2 i \pi}{3}}
$$

et il est réalisé par le feuilletage défini par

$$
\omega=d\left(y^{2}-x^{3}\right)+x(2 x d y-3 y d x) .
$$

Dans ce cas, on peut vérifier que

$$
d\left(\frac{\omega}{\left(y^{2}-x^{3}\right)^{1 / 6}}\right)=0
$$


et le groupoïde est de dimension 1. En fait, le pseudo-groupe de Lie transverse est le groupe engendré par $z \mapsto-j z$ et le groupe à un paramètre $z \mapsto z /(1-t z)$. Le revêtement $z^{6}=y^{2}-x^{3}$ est singulier; après un éclatement, il devient lisse et le diviseur exceptionnel est une courbe elliptique $C$ (isomorphe à $y^{2}-x^{3}=1$ ). De plus, $C$ est invariante par le feuilletage relevé $\widetilde{\mathcal{F}}$ qui a exactement une singularité de type col résonant. L'holonomie de $C$ est engendrée par les commutateurs

$$
\widetilde{G}=\left\langle\left[f_{1}, f_{2}\right],\left[f_{1}, f_{2}^{\circ 2}\right]\right\rangle .
$$

Le feuilletage $\widetilde{\mathcal{F}}$ est défini par la 1-forme fermée $\frac{\omega}{\left(y^{2}-x^{3}\right)^{1 / 6}}$.

\subsubsection{Holonomie affine et intégrales premières de type Liouville}

Théorème 8.25 (Casale). Le groupoïde de Galois $\operatorname{Gal}(\mathcal{F})$ du feuilletage est de dimension transverse $\leq 2$ si, et seulement si, il existe des 1 -formes méromorphes $\omega_{0}$ et $\omega_{1}$ satisfaisant

$$
\mathcal{F}=\mathcal{F}_{\omega_{0}}, \quad\left\{\begin{array}{l}
d \omega_{0}=\omega_{0} \wedge \omega_{1}, \\
d \omega_{1}=0
\end{array}\right.
$$

Dans ce cas, la fonction $F=e^{\int \omega_{1}}$ est facteur intégrant pour $\omega_{0}$, i.e. $d\left(\frac{\omega_{0}}{F}\right)=0$, et l'intégrale première $H=\int \omega_{0} F$ est à monodromie affine.

En fait, $H$ appartient à la classe de Liouville. On appelle extension liouvillienne du corps des fonctions méromorphes à l'origine de $\mathbb{C}^{2}$ toute extension de corps résultant d'un empilement fini d'extensions construites en rajoutant :

- une fonction algébrique sur le corps précédent,

- une primitive d'une forme différentielle à coefficients dans le corps précédent,

- l'exponentielle d'un élément du corps précédent.

On appelle fonction de Liouville toute fonction appartenant à une extension liouvillienne. En 1992, Michael F. Singer démontre dans [209] le :

Théorème 8.26 (Singer). Si une singularité de feuilletage $\mathcal{F}$ admet une intégrale première de Liouville, alors $\mathcal{F}$ est transversalement affine au sens précédent.

Michel Berthier et Frédéric Touzet ont montré dans [15] que les singularités réduites transversalement affines sont les singularités linéaires et les cols résonants et nœud-cols dont le chapelet de sphères est affine au sens du Théorème 4.52. Une singularité générale transversalement affine n'a donc, après résolution, que des singularités réduites du type précédent et tous ses pseudo-groupes d'holonomie projective sont résolubles. Dans [161], 
Emmanuel Paul dresse la liste des conditions nécessaires et suffisantes sur le pseudo-groupe (tel qu'il est décrit dans le Théorème 7.20) pour qu'un feuilletage non dicritique soit transversalement affine au sens précédent.

\subsubsection{Holonomie projective et intégrales premières de type Riccati}

Théorème 8.27 (Casale). Le groupoïde de Galois $\operatorname{Gal}(\mathcal{F})$ du feuilletage est de dimension transverse 3 si, et seulement si, il existe des 1-formes méromorphes $\omega_{0}, \omega_{1}$ et $\omega_{2}$ satisfaisant

$$
\mathcal{F}=\mathcal{F}_{\omega_{0}}, \quad\left\{\begin{array}{l}
d \omega_{0}=\omega_{0} \wedge \omega_{1}, \\
d \omega_{1}=\omega_{0} \wedge \omega_{2}, \\
d \omega_{2}=\omega_{1} \wedge \omega_{2} .
\end{array}\right.
$$

Dans ce cas, une intégrale première $H$ à monodromie projective se déduit par intégration schwarzienne; Guy Casale montre par ailleurs dans [46] que tout feuilletage admettant une intégrale première de type Riccati est transversalement projective au sens précédent. Frédéric Touzet donne dans [220] la liste des singularités réduites transversalement projectives : ce sont les singularités linéaires et les cols résonants et nœud-cols dont le chapelet de sphères est projectif au sens du Théorème 4.52. Une singularité générale transversalement projective n'a donc, après résolution, que des singularités réduite du type précédent et tous ses pseudo-groupes d'holonomie projective sont résolubles d'après le Théorème 4.27.

\subsection{Passage de coins}

Nous reprenons ici les idées développées dans [161] puis dans [33] en les adaptant à notre approche topologique des pseudo-groupes. Il s'agit de dégager des conditions nécessaires et suffisantes sur les singularités réduites et les groupes d'holonomie projective apparaissant le long du diviseur $D$ après résolution de la singularité $\mathcal{F}$, et sur la manière dont les dynamiques se recollent aux coins du diviseur pour que $\mathcal{F}$ soit transversalement projectif (au sens de la section 8.3.6).

Si un des pseudo-groupes d'holonomie projective $G_{i}$ est non résoluble, alors une conséquence immédiate du Théorème 4.27 est que la clôture topologique $\bar{G}$ du pseudo-groupe d'holonomie $G$ du feuilletage est de dimension infinie au moins sur une partie de la transversale; par suite, la dimension transverse du groupoïde de $\operatorname{Gal}(\mathcal{F})$, i.e. de $\bar{G}^{\text {Lie }}$, est infinie. En particulier, le feuilletage n'admet pas d'intégrale première raisonable et l'obstruction est ici de nature topologique : si une autre singularité $\mathcal{F}^{\prime}$ est conjuguée à $\mathcal{F}$ par un homéomorphisme, alors elle n'admet pas non plus d'intégrale première. En effet, l'homéomorphisme va conjuguer $G$ au pseudo-groupe 
$G^{\prime}$ de $\mathcal{F}^{\prime}$, et par suite leur clôtures $\bar{G}$ et $\bar{G}^{\prime}$ et les algèbres de Lie correspondantes $\mathcal{A}$ et $\mathcal{A}^{\prime}$ (voir preuve du Théorème 4.25); si $\mathcal{A}$ est de dimension infinie, alors $\mathcal{A}^{\prime}$ l'est aussi.

Lorsque tous les pseudo-groupes d'holonomie $G_{i}$ sont résolubles, le pseudogroupe (global) $G$ peut très bien être de dimension infinie selon la manière dont les pseudo-groupes $G_{i}$ se comportent entre eux après avoir "passé les coins", c'est-à-dire après conjugaison par les applications de Dulac. Nous allons examiner quelles sont les conditions pour que $G$ reste de dimension finie; bien souvent, ceci obligera $\bar{G}^{\text {Lie }}$ à être lui aussi de dimension finie.

\subsubsection{Passage des coins linéaires de type col ou hyper- bolique}

On se place en un coin, c'est-à-dire à l'intersection de deux composantes invariantes du diviseur $D$ après réduction de $\mathcal{F}$ et on se place dans des coordonnées locales $(x, y)$ dans lesquelle le feuilletage est donné par $x d y-$ $\alpha y d x$ avec $\alpha \in \mathbb{C} \backslash \mathbb{R}_{\geq 0}$. L'application de Dulac est donnée par

$$
\mathcal{D}: T_{x} \rightarrow T_{y} ; x \mapsto x^{\alpha} .
$$

Considérons deux sous-groupes $G_{x}, G_{y} \subset \operatorname{Diff}(\mathbb{C}, 0)$, ou plutôt les pseudogroupes induits respectivement sur $T_{x}$ et $T_{y}$. On peut par exemple penser que $G_{x}$ est le pseudo-groupe d'holonomie de la composante $\{x=0\}$ du diviseur $D$; en particulier, $G_{x}$ contient l'holonomie locale $x \mapsto e^{2 i \pi / \alpha} x$ de $\{x=0\}$. Plus généralement, nous serons amenés à considérer le cas où $G_{x}$ est un des pseudo-groupes de Lie considérés dans la section 4.5, ou un "sous-pseudo-groupe Zariski dense" avec toujours comme hypothèse qu'il contient l'holonomie locale $x \mapsto e^{2 i \pi / \alpha} x$.

L'application de Dulac $\mathcal{D}$ envoie la 1 -forme $\frac{d x}{x}$ sur la 1 -forme $\frac{d y}{y}$ et conjugue donc les pseudo-groupes linéaires respectifs :

$$
\mathbb{L}(x)=\left\{\varphi(x)=a x ; a \in \mathbb{C}^{*}\right\} \stackrel{\mathcal{D}_{*}}{\longrightarrow} \mathbb{L}(y)=\left\{\varphi(y)=a y ; a \in \mathbb{C}^{*}\right\} .
$$

Plus précisément, la dynamique de $\varphi(x)=a x$ sur un voisinage de 0 est envoyée sur l'ensemble des dynamiques $\mathcal{D}_{*} \varphi(y)=a^{-\alpha} y$ toutes définies sur le voisinage de 0 ; notons que les déterminations de $a^{-\alpha}$ se déduisent les unes des autres en composant par l'holonomie $y \mapsto e^{2 i \pi \alpha} y$ de $\{y=0\}$. Si le calcul est formellement le même pour un nœud linéaire, rappelons que la dynamique ne passe pas. Si les pseudo-groupes $G_{x}$ et $G_{y}$ sont tous deux linéaires, alors le pseudo-groupe engendré $\left\langle G_{x}, G_{y}\right\rangle$ est linéaire dans chacune des deux transversales.

La 1-forme $\frac{d x}{x^{\nu+1}}$ est envoyée par $\mathcal{D}$ sur $\frac{d y}{y^{-\frac{\nu}{\alpha}+1}}$ et par suite le pseudogroupe

$$
\mathbb{A}_{\nu}(x)=\left\{\varphi(x)=a x /\left(1+b x^{\nu}\right)^{1 / \nu} ; a \in \mathbb{C}^{*}, b \in \mathbb{C}\right\}
$$


des transformations qui laissent la droite $\mathbb{C} \cdot \frac{d x}{x^{\nu+1}}$ invariante est envoyé sur $\mathbb{A}_{-\frac{\nu}{\alpha}}(y)$. Même si $\nu$ est entier, ce n'est pas le cas de $\frac{\nu}{\alpha}$ en général; pour autant, l'image $\mathbb{A}_{-\frac{\nu}{\alpha}}(y)$ reste un pseudo-groupe de Lie au sens de la section 4.5 : lorsque $\nu$ n'est pas entier, les éléments non linéaires de $\mathbb{A}_{\nu}(x)(b \neq 0)$ sont des transformations multiformes. Par contre, notons et c'est important que si les éléments d'un pseudo-groupe $G_{x} \subset \mathbb{A}_{\nu}(x)$ ont un domaine de définition maximal, alors il en va de même pour les éléments de $\mathcal{D}_{*} G_{x}$ dans $\mathbb{A}_{-\underline{\nu}}(y)$; ici, maximal veut dire qu'il existe un ouvert $U \subset T_{x}$ tel que, si $\varphi: \stackrel{\alpha}{V} \rightarrow U, V \subset U$, est un élément de $\left.G_{x}\right|_{U}$ et si $\varphi^{\prime}: V^{\prime} \rightarrow U$, $V \subset V^{\prime} \subset U$, est un élément de $\mathbb{A}_{\nu}(x)$ prolongeant $\varphi$ à un ouvert plus grand $V^{\prime}$, alors $\varphi^{\prime} \in G_{x}$.

Si $G_{x} \subset \mathbb{A}_{\nu}(x)$ et $G_{y} \subset \mathbb{L}(y)$, alors le pseudo-groupe engendré satisfait

$$
<G_{x}, \mathcal{D}^{*} G_{y}>\subset \mathbb{A}_{\nu}(x) \text { et } \quad<\mathcal{D}_{*} G_{x}, G_{y}>\subset \mathbb{A}_{-\frac{\nu}{\alpha}}(y) .
$$

Supposons maintenant $G_{x} \subset \mathbb{A}_{\nu}(x)$ et $G_{y} \subset \mathbb{A}_{\mu}(y)$ avec $\nu, \mu \in \mathbb{C}^{*}$, en supposant que ni $G_{x}$, ni $G_{y}$ n'est dans $\mathbb{L}$, et supposons de plus $\alpha \notin \mathbb{Q}$. Alors la clôture $\overline{\left\langle\mathbb{A}_{\nu}, \mathcal{D}^{*} \mathbb{A}_{\mu}\right\rangle}$ du pseudo-groupe engendré sur $T_{x}$ est d'un des types suivants :

- de dimension infinie, ou

- contenue dans $\mathbb{A}_{\nu}$, ou

- contenue dans $\mathbb{P}_{\nu}=<\mathbb{A}_{\nu}, \mathbb{A}_{-\nu}>$

dont l'algèbre de Lie est $\mathbb{C}<x^{1-\nu} \partial_{x}, x \partial_{x}, x^{\nu+1} \partial_{x}>$. En effet, puisque $\alpha \notin \mathbb{Q}$ et $G_{x}$ non abélien $\left(G_{x} \not \subset \mathbb{L}\right)$, sa partie translation n'est pas discrète et l'algèbre de Lie associée $\mathcal{A}_{x}$ contient un élément non nul de la forme $c z^{\nu+1} \partial_{z}$; de même, $\mathcal{D}^{*} \mathcal{A}_{y}$ contient un élément $d z^{-\alpha \mu+1} \partial_{z}$. En travaillant avec les crochets de Lie de ces deux éléments comme dans la preuve du Théorème de Lie, on montre que l'algèbre engendrée est de dimension infinie sauf lorsque $\alpha \mu= \pm \nu$. Notons que $\mathcal{D}_{*} \mathbb{P}_{\nu}=\mathbb{P}_{\frac{\nu}{\alpha}}$. Lorsque $\alpha \in \mathbb{Q}_{<0}$, il se peut que $G_{x}$ et $G_{y}$ soient des sous-groupes discrets et l'analyse de ce cas semble plus délicate. De ces arguments, on déduit facilement :

Corollaire 8.28. Au voisinage d'un diviseur $D$ invariant par le feuilletage, si toutes les singularités sont de type col ou hyperbolique et si les coins sont linéarisables, l'un au moins n'admettant pas d'intégrale première, alors on est dans l'un des cas suivants :

$-G \subset \mathbb{L}$ sur toute transversale au diviseur et dans ce cas, toutes les singularités sont linéarisables;

$-G \subset \mathbb{A}_{\nu_{i}}$ sur toute transversale $T_{i}$ au diviseur, $\nu_{i} \in \mathbb{C}^{*}$, et au moins un des groupes d'holonomie projective est non abélien;

- $G \subset \mathbb{P}_{\nu_{i}}$ sur toute transversale $T_{i}$ au diviseur, $\nu_{i} \in \mathbb{C}^{*}$, et au moins deux des groupes d'holonomie projective sont non abéliens;

- $G$ est de dimension infinie (en tout point de toute transversale).

Démonstration. On raisonne comme dans la preuve du Théorème 8.22. Considérons dans $D$ les sous-arbres $D_{i}^{\text {hol }}$ maximaux dont les coins ont une 
intégrale première holomorphe. Alors $D$ est la réunion des $D_{i}^{\text {hol }}$ et ces derniers s'intersectent sur des coins sans intégrale première holomorphe. Sur un tel coin $D_{i}^{\text {hol }} \cap D_{j}^{\text {hol }}$, on considère les pseudo-groupes $G_{i}$ et $G_{j}$ induits respectivement par le feuilletage restreint aux voisinages de $D_{i}^{\text {hol }}$ et $D_{j}^{\text {hol }}$ : d'après le Théorème $8.9, G_{i}$ et $G_{j}$ sont induits par des sous-groupes de $\operatorname{Diff}(\mathbb{C}, 0)$ sur des revêtements finis. En relevant l'application de Dulac sur ces revêtements, on peut raisonner comme dans la discussion précédente. Puisque $G_{i} \subset \operatorname{Diff}(\mathbb{C}, 0)$ contient l'holonomie linéaire non périodique du coin, c'est un sous-groupe de $\mathbb{L}$ ou de $\mathbb{A}_{\nu}$ dès qu'il est résoluble (sinon, $\bar{G}_{i}$ est de dimension infinie et c'est terminé). En poussant les $G_{i}$ d'un sousarbre à un autre, on raisonne comme au-dessus et on récupère à chaque étape ou bien un sous-groupe de $\mathbb{P}_{\nu}$, et on peut continuer, ou bien un pseudo-groupe de dimension infinie, et c'est terminé.

\subsubsection{Passage des coins de type cols euclidiens}

On se place en un coin du diviseur $D$ où la singularité est de type $(\text { Eucl })_{\mathrm{col}}$ : dans des coordonnées convenables $(x, y)$, le feuilletage est donné par

$$
\omega=\frac{d u}{u^{k+1}}+\lambda \frac{d u}{u}-p \frac{d x}{x}, \quad u:=x^{p} y^{q}
$$

et les holonomies de ses courbes invariantes calculées dans les transversales respectives $T_{x}=\{y=1\}$ et $T_{y}=\{x=1\}$ sont données par

$$
g(x)=e^{-2 i \pi q / p} \exp \left(\frac{2 i \pi}{p} X_{k p, \lambda-1}\right) \quad \text { et } \quad f(y)=e^{-2 i \pi p / q} \exp \left(\frac{2 i \pi}{q} X_{k q, \lambda}\right) .
$$

où $X_{k, \mu}=\frac{z^{k+1}}{1+\mu z^{k}} \partial_{z}$. En fait, si l'on considère les restrictions

$$
\left.\omega\right|_{T_{x}}=p \frac{d x}{x^{k p+1}}+p(\lambda-1) \frac{d x}{x} \quad \text { et }\left.\quad \omega\right|_{T_{y}}=q \frac{d y}{y^{k q+1}}+q \lambda \frac{d y}{y}
$$

on déduit, en intégrant ces dernières les coordonnées de Leau

$$
\psi_{x}=-\frac{1}{k x^{k p}}+p(\lambda-1) \log (x) \quad \text { et } \quad \psi_{y}=-\frac{1}{k y^{k q}}+q \lambda \log (y) .
$$

Alors l'application de Dulac est donnée par

$$
\mathcal{D}=\left(\psi_{y}\right)^{\circ(-1)} \circ \psi_{x}: T_{x} \rightarrow T_{y}
$$

où les déterminations sont choisies de sorte que $\mathcal{D}$ soit asymptote à $x \mapsto$ $x^{p / q}$ en $x=0$. Notons que

$$
g^{\circ p}(x)=\exp \left(X_{k p, \lambda-1}\right) \quad \text { et } \quad f^{\circ q}(y)=\exp \left(X_{k q, \lambda}\right) .
$$


Les clôtures de Lie des holonomies sont respectivement données par

$$
\mathbb{E}_{k p, \lambda-1}(x) \text { et } \mathbb{E}_{k q, \lambda}(y) ;
$$

rappelons que $\mathbb{E}_{k, \mu}$ est homothétique à $\mathbb{E}_{k, 1}$ pour tout $\mu \neq 0$ et notons qu'au plus un des deux groupes précédents peut avoir un résidu $\mu$ trivial.

Comme dans la section précédente, nous allons considérer des pseudogroupes de Lie $G_{x}$ et $G_{y}$ sur les transversales $T_{x}$ et $T_{y}$ contenant les holonomies respectives du col euclidien, et dont la clôture de Lie est dans la liste des pseudo-groupes de Lie déjà rencontrés, à savoir $\mathbb{A}_{\nu}$ ou $\mathbb{P}_{\nu}, \nu \in \mathbb{C}^{*}$, ou $\mathbb{E}_{k, \mu}, k \in \mathbb{Z}_{>0}, \mu \in \mathbb{C}$. Nous allons examiner à quelles conditions le pseudo-groupe engendré, via l'application de Dulac, reste de dimension finie.

Lorsque $\lambda \neq 1$, le pseudo-groupe $G_{x}$ est nécessairement contenu dans $\mathbb{E}_{k p, \lambda-1}$. En effet, les seuls difféomorphismes résonants contenus dans $\mathbb{A}_{\nu}$ ou $\mathbb{P}_{\nu}$ sont $a \exp \left(X_{\nu, 0}\right)$, pour $\nu \in \mathbb{Z}_{>0}, a^{\nu}=1$, et sont de résidu nul. L'image par $\mathcal{D}$ est alors un sous-groupe de $\mathbb{E}_{k q, \lambda}$. Par contre, lorsque $\lambda=1$, alors $G_{x}$ peut aussi être contenu dans $\mathbb{A}_{k p}$ ou dans $\mathbb{P}_{k p}$. Les images de ces deux pseudo-groupes par $\psi_{x}$ sont les pseudo-groupes $\mathbb{A}_{1}$ et $\mathbb{P}_{1}$ des transformations affines $w \mapsto a w+b$ et projectives $w \mapsto \frac{a w+b}{c w+d}$ respectivement. Il nous faut maintenant comprendre quels sont les pseudo-groupes $\left(\psi_{y}\right)^{*} \mathbb{A}_{1}$ et $\left(\psi_{y}\right)^{*} \mathbb{P}_{1}$. Plus généralement, considérons $\psi(z)=-\frac{1}{k z^{k+1}}+\mu \log (z)$; alors

$$
\mathbb{A}_{k, \mu}:=\psi^{*} \mathbb{A}_{1}=\left\{\varphi(z) ; f \circ \varphi \cdot \varphi^{\prime}+\frac{\varphi^{\prime \prime}}{\varphi^{\prime}}=f\right\}
$$

où $f=\frac{\psi^{\prime \prime}}{\psi^{\prime}}$. En effet, les éléments de $\mathbb{A}_{1}\left(\right.$ resp. $\left.\mathbb{A}_{k, \mu}\right)$ sont caractérisés par le fait qu'ils préservent la droite $\mathbb{C} \cdot d w($ resp. $\mathbb{C} \cdot d \psi)$; ainsi

$$
\varphi \in \mathbb{A}_{k, \mu} \quad \Leftrightarrow \quad \psi^{\prime} \circ \varphi \cdot \varphi^{\prime}=c_{\varphi} \cdot \psi^{\prime} \quad \Leftrightarrow \quad \frac{\psi^{\prime \prime} \circ \varphi \cdot \varphi^{\prime}}{\psi^{\prime} \circ \varphi}+\frac{\varphi^{\prime \prime}}{\varphi^{\prime}}=\frac{\psi^{\prime \prime}}{\psi^{\prime}} .
$$

De la même manière, on montre (voir [47]) que

$$
\mathbb{P}_{k, \mu}:=\psi^{*} \mathbb{P}_{1}=\left\{\varphi(z) ; g \circ \varphi \cdot\left(\varphi^{\prime}\right)^{2}+\mathcal{S}(\varphi)=g\right\}
$$

où $\mathcal{S}(\varphi)=\frac{\varphi^{\prime \prime \prime}}{\varphi^{\prime}}-\frac{3}{2}\left(\frac{\varphi^{\prime \prime}}{\varphi^{\prime}}\right)^{2}$ est la dérivée Schwartzienne et $g=\mathcal{S}(\psi)$. Une chose importante à retenir pour la suite est que $f=-\frac{k+1+\mu z^{k}}{z\left(1+\mu z^{k}\right)}$ et $g=$ $f^{\prime}-\frac{1}{2} f^{2}$ sont méromorphes en $z=0$ et donc que $\mathbb{A}_{k, \mu}$ et $\mathbb{P}_{k, \mu}$ sont des pseudo-groupes de Lie de dimension respective 2 et 3 au sens de Malgrange (voir [47]). Maintenant, il est clair que l'application de Dulac $\mathcal{D}$ définie plus haut conjugue les pseudo-groupes de type affine

$$
\mathbb{A}_{k p, \lambda-1} \quad \text { et } \quad \mathbb{A}_{k q, \lambda}
$$

ainsi que les pseudo-groupes de type projectif

$$
\mathbb{P}_{k p, \lambda-1} \quad \text { et } \quad \mathbb{P}_{k q, \lambda} .
$$


Corollaire 8.29. Au voisinage d'un diviseur $D$ invariant par le feuilletage, si toutes les singularités sont de type col ou hyperbolique et si les coins sont ou bien linéarisables, ou bien euclidiens normalisables, l'un au moins n'admettant pas d'intégrale première holomorphe, alors on est dans l'un des cas suivants :

$-G \subset \mathbb{L}$ sur toute transversale au diviseur et dans ce cas, toutes les singularités sont linéarisables;

- $G \subset \mathbb{E}_{k_{i}, \mu_{i}}$ sur toute transversale $T_{i}$ au diviseur et dans ce cas, toutes les singularités sont résonantes, ou à intégrale première holomorphe;

- $G \subset \mathbb{A}_{\nu_{i}}$ ou $\mathbb{A}_{k_{i}, \mu_{i}}$ sur toute transversale $T_{i}$ au diviseur, $\nu_{i}, \mu_{i} \in \mathbb{C}^{*}$, $k_{i} \in \mathbb{N}^{*}$, et au moins un des groupes d'holonomie projective est non abélien;

- $G \subset \mathbb{P}_{\nu_{i}}$ ou $\mathbb{P}_{k_{i}, \mu_{i}}$ sur toute transversale $T_{i}$ au diviseur, $\nu_{i}, \mu_{i} \in \mathbb{C}^{*}$, $k_{i} \in \mathbb{N}^{*}$, et au moins deux des groupes d'holonomie projective sont non abéliens;

- $G$ est de dimension topologique infinie (en tout point de toute transversale).

Démonstration. On procède comme dans la preuve du Corollaire 8.28. On considère cette fois-ci le recouvrement de $D$ par les sous-arbres maximaux $D_{i}^{\text {lin }}$ à coins linéaires. Si $D_{i}^{\text {lin }}=D$, on est ramené au Corollaire 8.28. Sinon, notons $G_{i}$ le pseudo-groupe d'holonomie du feuilletage restreint au voisinage de $D_{i}^{\text {lin }}:$ il est de l'une des formes données par le Corollaire 8.28 ou le Théorème 8.9. Par exemple, si un des $G_{i}$ est de dimension infinie, il l'est en tout point de la transversale et cette propriété se propage sur toute transversale à $D$. Supposons donc chaque $G_{i}$ de dimension finie. Sur un coin euclidien $D_{i}^{\text {lin }} \cap D_{j}^{\text {lin }}$ on voit facilement avec les notations précédentes que le pseudo-groupe $G_{i}$ correspondant à $D_{i}^{\text {lin }}$ ne peut être que du type $\mathbb{E}_{k p, \lambda-1}$ si $\lambda \neq 1$ ou encore du type $\mathbb{A}_{k p}$ ou $\mathbb{P}_{k p}$ lorsque $\lambda=1$. Pour cela, on utilise le fait que $G_{i}$ contient l'holonomie locale $g^{\circ p}(x)=\exp \left(X_{k p, \lambda-1}\right)$. Par exemple, si $D_{i}^{\text {lin }}$ est à coins holomorphes, le Théorème 8.9 nous dit que $G_{i}$ se relève en un sous-groupe $\tilde{G}_{i} \subset \operatorname{Diff}(\mathbb{C}, 0)$ sur un revêtement fini, disons dans la variable $z=x^{1 / d} ; \tilde{G}_{i}$ contient alors le relevé $\tilde{g}^{\circ p}(z)=$ $\exp \left(d X_{k p d, \lambda-1}\right)$ et est nécessairement du type $\mathbb{E}_{k p d, \lambda-1} ;$ par suite, $G_{i} \subset$ $\mathbb{E}_{k p, \lambda-1} \subset \operatorname{Diff}(\mathbb{C}, 0)$ (le revêtement n'était pas nécessaire ici). Maintenant, si un des coins de $D_{i}^{\text {lin }}$ n'est pas holomorphe, le Corollaire 8.28 nous dit que $G_{i}$ est analytiquement conjugué à un des modèles $\mathbb{L}, \mathbb{A}_{\nu}$ ou $\mathbb{P}_{\nu}, \nu \in \mathbb{C}^{*}$; mais puisque $G_{i}$ contient $\exp \left(X_{k p, \lambda-1}\right)$, la seule possibilité est $\nu=k p$ et $\lambda-1=0$ ( $\mathbb{L}$ est exclu) et $G_{i} \subset \mathbb{A}_{\nu}$ ou $\mathbb{P}_{\nu}$ dans la coordonnée $x$. Dans tous les cas, on observe que les dynamiques $G_{i}$ et $G_{j}$ engendrent encore un pseudo-groupe de dimension finie qui sur toute transversale voisine d'un coin euclidien est un sous-pseudo-groupe de $\mathbb{P}_{k_{i}, \mu_{i}}$. On observe a posteriori que, si les $G_{i}$ sont de type euclidien (resp. affine, projectif), alors le pseudogroupe global $G$ est aussi de type euclidien (resp. affine, projectif). 


\subsubsection{Passage des coins de type $(\mathrm{Lin})_{\mathrm{col}}^{\mathrm{ex}}$ et $(\mathrm{Eucl})_{\mathrm{col}}^{\mathrm{ex}}$}

Un col de type (Lin) col $_{\text {col }}^{\text {ex }}$ est formellement linéarisable mais non analytiquement linéarisable. Ses holonomies sont des dynamiques de hérisson de type $(\operatorname{Lin})^{\text {ex}}$. Pour un tel coin $D_{i} \cap D_{j}$, l'holonomie $G_{i} \subset \operatorname{Diff}(\mathbb{C}, 0)$ de $D_{i}$ est ou bien de type (Lin) ${ }^{\text {ex }}$, ou bien non résoluble (et $G_{i}$ est de dimension infinie en tout point de la transversale). Dans le premier cas, on déduit que toutes les singularités de $D_{i}$ doivent être de type (Lin) $)_{\text {col }}^{\text {ex }}$ ou (Fin) $)_{\text {col }}$. De plus, puisque $G_{i}$ commute à l'holonomie, son image par l'application de Dulac est encore un sous-groupe de $\operatorname{Diff}(\mathbb{C}, 0)$ de type $(\operatorname{Lin})^{\text {ex }}$. En raisonnant sur les sous-arbre maximaux $D_{i}^{\text {hol }}$ à coins holomorphes, on montre de proche en proche que toutes les singularités sont de type (Lin) ${ }_{\mathrm{col}}^{\mathrm{ex}}$ ou (Fin) col le long de $D$ dès que le pseudo-groupe global $G$ n'est pas de dimension infinie. Alors $G$ calculé sur n'importe quelle transversale à $D$ est (à revêtement ramifié près) un sous-groupe de $\operatorname{Diff}(\mathbb{C}, 0)$ de type $(\operatorname{Lin})^{\text {ex }}$.

Un col de type (Eucl) ${ }_{\mathrm{col}}^{\mathrm{ex}}$ est formellement mais non analytiquement équivalent à un col euclidien. Pour un tel coin $D_{i} \cap D_{j}$, l'holonomie $G_{i} \subset$ $\operatorname{Diff}(\mathbb{C}, 0)$ de $D_{i}$ est ou bien de type (Eucl) ${ }^{\text {ex }}$ ou (Aff) ${ }^{\text {ex }}$, ou bien non résoluble. On déduit comme précédemment, dès que $G$ n'est pas de dimension infinie, qu'il est un sous-groupe de $\operatorname{Diff}(\mathbb{C}, 0)$ de type $(\text { Eucl })^{\text {ex }}$ ou $(\text { Aff })^{\text {ex }}$ sur n'importe quelle transversale à $D$ et que tout point singulier le long de $D$ est de type $(\text { Eucl })_{\text {col }}^{\text {ex }}$ ou (Fin) $)_{\mathrm{col}}$.

Finalement, en utilisant le résultat principal de [48], nous déduisons de notre étude le

Théorème 8.30. Soit $\mathcal{F}$ une singularité de feuilletage. On suppose que $\mathcal{F}$ est non dicritique et que toute singularité réduite apparaissant le long du diviseur exceptionnel $D$ après résolution est de type col ou hyperbolique (pas de nœud, ni de nœud-col). Considérons le pseudo-groupe d'holonomie $(T, G)$ du feuilletage restreint au complémentaire de la courbe invariante $C$. Alors on est dans l'une des situations suivantes:

- la cloture $\bar{G}$ contient le pseudo-groupe de toutes les transformations conformes sur un ouvert de Zariski réel de $T$,

- $\mathcal{F}$ est transversalement projectif, i.e. $\operatorname{Gal}(\mathcal{F})$ est de dimension transverse $\leq 3$,

- $G$ est de type $(\mathrm{Lin})^{\mathrm{ex}}$ et toutes les singularités réduites sont de type (Lin) $)_{\mathrm{col}}^{\mathrm{ex}}$ ou (Fin) $)_{\mathrm{col}}$, l'une au moins étant du premier type,

- $G$ est de type (Eucl) ${ }^{\mathrm{ex}}$ ou (Aff) ${ }^{\mathrm{ex}}$ et toutes les singularités réduites sont de type $(\mathrm{Eucl})_{\mathrm{col}}^{\mathrm{ex}}$ ou $(\mathrm{Fin})_{\mathrm{col}}$.

Dans les deux derniers cas, le pseudo-groupe $G$ calculé sur n'importe quelle transversale $T$ au diviseur est induit par un sous-groupe de $\operatorname{Diff}(\mathbb{C}, 0)$.

En fait, le second cas correspond au cas où $G$ est un des pseudo-groupes de dimension finie listés dans le Corollaire 8.29. Pour montrer que $\mathcal{F}$ est transversalement projectif (resp. affine, linéaire ou euclidien), il faut encore montrer que $\operatorname{Gal}(\mathcal{F})$ est de dimension transverse $\leq 3$ en dehors des 
singularités, c'est-à-dire aussi sur une transversale à chaque courbe invariante. Le raisonnement est alors le même qu'en un coin. Ceci étant fait, on déduit que $\mathcal{F}$ est transversalement projectif en invoquant [48].

Dans le troisième cas, rappelons que la clôture de Lie $\bar{G}^{\text {Lie }}$ est de dimension infinie. La dynamique étant ici de type hérisson, tout feuilletage topologiquement conjugué à $\mathcal{F}$ sera du même type.

Dans le quatrième cas, il se peut que le feuilletage soit transversalement projectif (voir Théorème 4.52). Mais la clôture de Lie $\bar{G}^{\mathrm{Lie}}$ du pseudogroupe $G$ est plus dégénérée en 0 que les pseudo-groupes de Lie apparaissant dans le Corollaire 8.29. Rappelons par ailleurs qu'un pseudo-groupe $G$ de type (Eucl) ${ }^{\text {ex }}$ ou (Aff) ${ }^{\text {ex }}$ est topologiquement conjugué à un pseudogroupe $G_{0}$ de type (Eucl) ou (Aff). On peut en fait construire un feuilletage $\mathcal{F}_{0}$ ayant même réduction que $\mathcal{F}$, topologiquement conjugué à $\mathcal{F}$ et réalisant $G_{0}$. De ce fait, le troisième cas du Théorème 8.30 n'est pas topologiquement bien défini. Mais $G$ comme $G_{0}$ ont ceci de très particulier que le quotient (l'espace des feuilles) est un chapelet de sphères; ceci caractérise les pseudo-groupes comme $G$ ou $G_{0}$.

Ainsi, nous obtenons par exemple le :

Corollaire 8.31. Soit $\mathcal{F}$ comme dans le Théorème 8.30. Si $\mathcal{F}$ contient au moins une singularité non résonante $(\alpha \notin \mathbb{Q})$ ou si l'algèbre de Lie $\mathcal{A}$ associée à $\bar{G}$ est non triviale, alors toute autre singularité $\mathcal{F}^{\prime}$ topologiquement conjuguée à $\mathcal{F}$ a le même type d'intégrale première que $\mathcal{F}$, i.e. $\operatorname{Gal}(\mathcal{F})$ et $\operatorname{Gal}\left(\mathcal{F}^{\prime}\right)$ ont même dimension transverse.

Exercice 8.32. Construire un exemple de feuilletage comme dans le Théorème 8.30 dont le pseudo-groupe est de dimension topologique infinie et tous les groupes d'holonomie projective sont abéliens.

Exercice 8.33. Montrer que le Théorème 8.30 reste vrai si l'on autorise des nœuds linéaires sur la partie lisse de D. Montrer qu'il devient faux dès que l'on autorise les nœuds dans les coins.

Exercice 8.34. Construire 5 feuilletages dicritiques topologiquement conjugués dont le groupoïde de Galois est de dimension respective 0, 1, 2, 3 et $\infty$.

Exercice 8.35. Que se passe t'il si l'on autorise les nœud-cols sur la partie lisse du diviseur $D$ avec variété centrale transverse à $D$, contenue dans $D$ ou encore dans un coin? 


\section{Bibliographie}

[1] M. Abate. Open problems in local discrete holomorphic dynamics. Anal. Math. Phys., 1(4) :261-287, 2011.

[2] N. H. Abel. Euvres complètes. Tome II. Éditions Jacques Gabay, Sceaux, 1992. Suivi de "Niels Henrik Abel : sa vie et son action scientifique" par C.-A. Bjerknes. [Followed by "Niels Henrik Abel : his life and his scientific activity" by C.-A. Bjerknes] (1884), Edited and with notes by L. Sylow and S. Lie, Reprint of the second (1881) edition.

[3] P. Ahern and X. Gong. A complete classification for pairs of real analytic curves in the complex plane with tangential intersection. $J$. Dyn. Control Syst., 11(1) :1-71, 2005.

[4] P. Ahern and J.-P. Rosay. Entire functions, in the classification of differentiable germs tangent to the identity, in one or two variables. Trans. Amer. Math. Soc., 347(2) :543-572, 1995.

[5] D. S. Alexander. A history of complex dynamics. Aspects of Mathematics, E24. Friedr. Vieweg \& Sohn, Braunschweig, 1994. From Schröder to Fatou and Julia.

[6] I. N. Baker. Permutable power series and regular iteration. J. Austral. Math. Soc., 2 :265-294, 1961/1962.

[7] I. N. Baker. Fractional iteration near a fixpoint of multiplier 1. J. Austral. Math. Soc., 4 :143-148, 1964.

[8] I. N. Baker. Non-embeddable functions with a fixpoint of multiplier 1. Math. Z., 99 :377-384, 1967.

[9] W. Balser. From divergent power series to analytic functions, volume 1582 of Lecture Notes in Mathematics. Springer-Verlag, Berlin, 1994. Theory and application of multisummable power series.

[10] M. Belliart, I. Liousse, and F. Loray. Sur l'existence de points fixes attractifs pour les sous-groupes de Aut $(\mathbb{C}, 0)$. C. R. Acad. Sci. Paris Sér. I Math., 324(4) :443-446, 1997. 
[11] M. Belliart, I. Liousse, and F. Loray. Conformal flows on $(\mathbb{C}, 0)$ and hexagonal 3-webs. In Web theory and related topics (Toulouse, 1996), pages 92-105. World Sci. Publishing, River Edge, NJ, 2001.

[12] M. Belliart, I. Liousse, and F. Loray. The generic rational differential equation $d w / d z=P_{n}(z, w) / Q_{n}(z, w)$ on $\mathbb{C P}^{2}$ carries no interesting transverse structure. Ergodic Theory Dynam. Systems, 21(6) :1599$1607,2001$.

[13] M. Berthier, D. Cerveau, and A. Lins Neto. Sur les feuilletages analytiques réels et le problème du centre. J. Differential Equations, 131(2) :244-266, 1996.

[14] M. Berthier, R. Meziani, and P. Sad. On the classification of nilpotent singularities. Bull. Sci. Math., 123(5) :351-370, 1999.

[15] M. Berthier and F. Touzet. Sur l'intégration des équations différentielles holomorphes réduites en dimension deux. Bol. Soc. Brasil. Mat. (N.S.), 30(3) :247-286, 1999.

[16] G. D. Birkhoff. Surfaces transformations and their dynamical applications. Acta Math., 43 :1-119, 1922.

[17] G. D. Birkhoff. Déformations analytiques et fonctions autoéquivalentes. Ann. Inst. H. Poincaré, 9 :51-122, 1939.

[18] P. Blanchard. Complex analytic dynamics on the Riemann sphere. Bull. Amer. Math. Soc. (N.S.), 11(1) :85-141, 1984.

[19] L. E. Böttcher. The principal laws of convergence of iterates and their application to analysis (russe). Izv. Kazan. Fiz.-Mat. Obshch., $14: 155-234,1904$.

[20] E. Brieskorn and H. Knörrer. Plane algebraic curves. Birkhäuser Verlag, Basel, 1986. Translated from the German by John Stillwell.

[21] A. D. Brjuno. Analytic form of differential equations. I, II. Trudy Moskov. Mat. Obšč, 25 :119-262 ; ibid. 26 (1972), 199-239, 1971.

[22] F. E. Brochero Martínez. Groups of germs of analytic diffeomorphisms in $\left(\mathbb{C}^{2}, 0\right)$. J. Dynam. Control Systems, 9(1) :1-32, 2003.

[23] M. Brunella. Some remarks on indices of holomorphic vector fields. Publ. Mat., 41(2) :527-544, 1997.

[24] M. Brunella. Birational geometry of foliations. Monografías de Matemática. [Mathematical Monographs]. Instituto de Matemática Pura e Aplicada (IMPA), Rio de Janeiro, 2000. Available electronically at http ://www.impa.br/Publicacoes/Monografias/Abstracts/brunella.ps.

[25] G. Calsamiglia and Y. Genzmer. Classification of regular dicritical foliations. Ergodic Theory Dynam. Systems, 37(5) :1443-1479, 2017. 
[26] G. Calsamiglia-Mendlewicz. Finite determinacy of dicritical singularities in $\left(\mathbb{C}^{2}, 0\right)$. Ann. Inst. Fourier (Grenoble), 57(2) :673-691, 2007.

[27] C. Camacho. On the local structure of conformal mappings and holomorphic vector fields in $\mathbb{C}^{2}$. In Journées Singulières de Dijon (Univ. Dijon, Dijon, 1978), volume 59 of Astérisque, pages 3, 83-94. Soc. Math. France, Paris, 1978.

[28] C. Camacho. Quadratic forms and holomorphic foliations on singular surfaces. Math. Ann., 282(2) :177-184, 1988.

[29] C. Camacho. Dicritical singularities of holomorphic vector fields. In Laminations and foliations in dynamics, geometry and topology (Stony Brook, NY, 1998), volume 269 of Contemp. Math., pages 3945. Amer. Math. Soc., Providence, RI, 2001.

[30] C. Camacho and B. Azevedo Scárdua. The transcendence of solutions of complex differential equations. In J. Mozo Fernandez, editor, Ecuationes diferenciales y singularidades, pages 79-102. Universidad de Valladolid, 1995.

[31] C. Camacho and B. Azevedo Scárdua. Beyond Liouvillian transcendence. Math. Res. Lett., 6(1) :31-41, 1999.

[32] C. Camacho and B. Azevedo Scárdua. Complex foliations with algebraic limit sets. Astérisque, (261) :xi, 57-88, 2000. Géométrie complexe et systèmes dynamiques (Orsay, 1995).

[33] C. Camacho and B. Azevedo Scárdua. Holomorphic foliations with Liouvillian first integrals. Ergodic Theory Dynam. Systems, 21(3) :717-756, 2001.

[34] C. Camacho and B. Azevedo Scárdua. Erratum to : "Holomorphic foliations with Liouvillian first integrals" [Ergodic Theory Dynam. Systems 21 (2001), no. 3, 717-756 ; MR1836428 (2002k :37080)]. Ergodic Theory Dynam. Systems, 23(3) :985-987, 2003.

[35] C. Camacho and A. Lins Neto. The topology of integrable differential forms near a singularity. Inst. Hautes Etudes Sci. Publ. Math., (55) :5-35, 1982.

[36] C. Camacho and A. Lins Neto. Geometric theory of foliations. Birkhäuser Boston, Inc., Boston, MA, 1985. Translated from the Portuguese by Sue E. Goodman.

[37] C. Camacho, A. Lins Neto, and P. Sad. Topological invariants and equidesingularization for holomorphic vector fields. J. Differential Geom., 20(1) :143-174, 1984.

[38] C. Camacho and P. Sad. Invariant varieties through singularities of holomorphic vector fields. Ann. of Math. (2), 115(3) :579-595, 1982. 
[39] M. Canalis-Durand and R. Schäfke. Divergence and summability of normal forms of systems of differential equations with nilpotent linear part. Ann. Fac. Sci. Toulouse Math. (6), 13(4) :493-513, 2004.

[40] J. C. Canille Martins. Holomorphic flows in $\mathbf{C}^{3}, 0$ with resonances. Trans. Amer. Math. Soc., 329(2) :825-837, 1992.

[41] F. Cano and D. Cerveau. Desingularization of nondicritical holomorphic foliations and existence of separatrices. Acta Math., 169(12) :1-103, 1992.

[42] F. Cano, D. Cerveau, and J. Déserti. Téorie élémentaire des feuilletages holomorphes singuliers. Éditions Belin, Paris, 2013.

[43] J. Cano. An extension of the Newton-Puiseux polygon construction to give solutions of Pfaffian forms. Ann. Inst. Fourier (Grenoble), 43(1): :125-142, 1993.

[44] F. Cano Torres. Desingularization strategies for three-dimensional vector fields, volume 1259 of Lecture Notes in Mathematics. SpringerVerlag, Berlin, 1987.

[45] S. Cantat, D. Cerveau, V. Guirardel, and J. Souto. Surface groups in the group of germs of analytic diffeomorphisms in one variable. Enseign. Math., 66(1-2) :93-134, 2020.

[46] G. Casale. Suites de Godbillon-Vey et intégrales premières. C. R. Math. Acad. Sci. Paris, 335(12) :1003-1006, 2002.

[47] G. Casale. $\mathcal{D}$-enveloppe d'un difféomorphisme de ( $\mathbb{C}, 0)$. Ann. Fac. Sci. Toulouse Math. (6), 13(4) :515-538, 2004.

[48] G. Casale. Feuilletages singuliers de codimension un, groupoïde de Galois et intégrales premières. Ann. Inst. Fourier (Grenoble), 56(3) :735-779, 2006.

[49] G. Casale. Simple meromorphic functions are algebraic. Bull. Braz. Math. Soc. (N.S.), 44(2) :309-319, 2013.

[50] D. Cerveau. Densité des feuilles de certaines équations de Pfaff à 2 variables. Ann. Inst. Fourier (Grenoble), 33(1) :185-194, 1983.

[51] D. Cerveau. Une liste de problèmes. In J. Mozo Fernandez, editor, Ecuationes diferenciales y singularidades, pages 455-460. Universidad de Valladolid, 1995.

[52] D. Cerveau and A. Lins Neto. Frobenius theorem for foliations on singular varieties. Bull. Braz. Math. Soc. (N.S.), 39(3) :447-469, 2008.

[53] D. Cerveau and A. Lins Neto. Codimension two holomorphic foliations. J. Differential Geom., 113(3) :385-416, 2019.

[54] D. Cerveau, A. Lins Neto, F. Loray, J. V. Pereira, and F. Touzet. Complex codimension one singular foliations and Godbillon-Vey sequences. Mosc. Math. J., 7(1) :21-54, 166, 2007. 
[55] D. Cerveau, A. Lins Neto, and M. Ravara-Vago. Feuilletages holomorphes de codimension 1 : une étude locale dans le cas dicritique. Ann. Sci. Éc. Norm. Supér. (4), 49(1) :213-247, 2016.

[56] D. Cerveau and F. Loray. Un théorème de Frobenius singulier via l'arithmétique élémentaire. J. Number Theory, 68(2) :217-228, 1998.

[57] D. Cerveau and J.-F. Mattei. Formes intégrables holomorphes singulières, volume 97 of Astérisque. Société Mathématique de France, Paris, 1982. With an English summary.

[58] D. Cerveau and R. Moussu. Groupes d'automorphismes de $(\mathbb{C}, 0)$ et équations différentielles $y d y+\cdots=0$. Bull. Soc. Math. France, 116(4) :459-488 (1989), 1988.

[59] D. Cerveau and J. Mozo-Fernández. Classification analytique des feuilletages singuliers réduits de codimension 1 en dimension $n \geq 3$. Ergodic Theory Dynam. Systems, 22(4) :1041-1060, 2002.

[60] D. Cerveau and P. Sad. Problèmes de modules pour les formes différentielles singulières dans le plan complexe. Comment. Math. Helv., 61(2) :222-253, 1986.

[61] D. Cerveau and P. Sad. Liouvillian integration and Bernoulli foliations. Trans. Amer. Math. Soc., 350(8) :3065-3081, 1998.

[62] S. D. Cohen. The group of translations and positive rational powers is free. Quart. J. Math. Oxford Ser. (2), 46(181) :21-93, 1995.

[63] N. Corral. Sur la topologie des courbes polaires de certains feuilletages singuliers. Ann. Inst. Fourier (Grenoble), 53(3) :787-814, 2003.

[64] H. Cremer. Zum Zentrumproblem. Math. Ann., 98(1) :151-163, 1928.

[65] H. Cremer. Über die Häufigkeit der Nichtzentren. Math. Ann., 115(1):573-580, 1938.

[66] A. A. Diaw and F. Loray. Pairs of foliations and Mattei-Moussu's Theorem. Bull. Sci. Math., 171 :103035, 2021.

[67] A. Dudko and D. Sauzin. The resurgent character of the Fatou coordinates of a simple parabolic germ. C. R. Math. Acad. Sci. Paris, 352(3):255-261, 2014.

[68] A. Dudko and D. Sauzin. On the resurgent approach to ÉcalleVoronin's invariants. C. R. Math. Acad. Sci. Paris, 353(3) :265-271, 2015.

[69] H. Dulac. Recherches sur les points singuliers des équations différentielles. J. École Polytechnique, 2 :1-125, 1904.

[70] J. Écalle. Théorie itérative : introduction à la théorie des invariants holomorphes. J. Math. Pures Appl. (9), 54 :183-258, 1975. 
[71] J. Écalle. Les fonctions résurgentes. Tome II, volume 6 of Publications Mathématiques d'Orsay 81 /Mathematical Publications of Orsay 81]. Université de Paris-Sud Département de Mathématique, Orsay, 1981. Les fonctions résurgentes appliquées à l'itération. [Resurgent functions applied to iteration].

[72] J. Écalle and B. Vallet. Intertwined mappings. Ann. Fac. Sci. Toulouse, XIII(3) :291-376, 2004.

[73] P. M. Elizarov. The orbital topological classification of analytic differential equations in a neighborhood of a degenerate elementary singular point in the two-dimensional complex plane. Trudy Sem. Petrovsk., (13) :137-165, 257, 1988.

[74] P. M. Elizarov, Yu. S. Ilyashenko, A. A. Shcherbakov, and S. M. Voronin. Finitely generated groups of germs of one-dimensional conformal mappings, and invariants for complex singular points of analytic foliations of the complex plane. In Nonlinear Stokes phenomena, volume 14 of Adv. Soviet Math., pages 57-105. Amer. Math. Soc., Providence, RI, 1993.

[75] P. Erdôs and E. Jabotinsky. On analytic iteration. J. Analyse Math., 8 :361-376, 1960/1961.

[76] J. Farkas. Sur les fonctions itératives. Jour. Math. Pures et Appl. Série 3, 10 :101-108, 1884.

[77] P. Fatou. Sur les équations fonctionnelles. Bull. Soc. Math. France, 47 :161-271, 1919.

[78] B. Fine, F. Levin, and G. Rosenberger. Free subgroups and decompositions of one-relator products of cyclics. I. The Tits alternative. Arch. Math. (Basel), 50(2):97-109, 1988.

[79] Y. Genzmer. Rigidity for dicritical germ of foliation in $\mathbb{C}^{2}$. Int. Math. Res. Not. IMRN, (19) :Art. ID rnm072, 14, 2007.

[80] Y. Genzmer. Un problème de Riemann-Hilbert local non-linéaire pour les germes de feuilletages holomorphes singuliers en dimension deux. C. R. Math. Acad. Sci. Paris, 344(1) :45-48, 2007.

[81] Y. Genzmer. Analytical and formal classifications of quasihomogeneous foliations in $\left(\mathbb{C}^{2}, 0\right)$. J. Differential Equations, $245(6): 1656-1680,2008$.

[82] Y. Genzmer. Construction of foliations with prescribed separatrix. Ergodic Theory Dynam. Systems, 28(3) :817-842, 2008.

[83] Y. Genzmer. Classification of absolutely dicritical foliations of cusp type. Publ. Mat., 57(2) :333-357, 2013.

[84] A. Glutsyuk. On the monodromy group of confluent linear equations. Mosc. Math. J., 5(1) :67-90, 2005. 
[85] C. Godbillon. Feuilletages, volume 98 of Progress in Mathematics. Birkhäuser Verlag, Basel, 1991. Études géométriques. [Geometric studies], With a preface by G. Reeb.

[86] X. Gómez-Mont and I. Luengo. Germs of holomorphic vector fields in $\mathbb{C}^{3}$ without a separatrix. Invent. Math., 109(2) :211-219, 1992.

[87] X. Gómez-Mont and B. Wirtz. On fixed points of conformal pseudogroups. Bol. Soc. Brasil. Mat. (N.S.), 26(2) :201-209, 1995.

[88] H. Grauert. Über Modifikationen und exzeptionelle analytische Mengen. Math. Ann., 146 :331-368, 1962.

[89] J. Hadamard. Two works on iteration and related questions. Bull. Amer. Math. Soc., 50 :67-75, 1944.

[90] M. Hukuhara, T. Kimura, and T. Matuda. Equations différentielles ordinaires du premier ordre dans le champ complexe. Publications of the Mathematical Society of Japan, 7. The Mathematical Society of Japan, Tokyo, 1961.

[91] Y. S. Il'yashenko. Topology of phase portraits of analytic differential equations on a complex projective plane. Trudy Sem. Petrovsk., (4) :83-136, 1978.

[92] Y. S. Il'yashenko, editor. Nonlinear Stokes phenomena, volume 14 of Advances in Soviet Mathematics. American Mathematical Society, Providence, RI, 1993.

[93] Y. S. Il'yashenko. The nonlinear Riemann-Hilbert problem. Tr. Mat. Inst. Steklova, 213(Differ. Uravn. s Veshchestv. i Kompleks. Vrem.) :10-34, 1997.

[94] Y. S. Ilyashenko and S. Yakovenko. Lectures on analytic differential equations, volume 86 of Graduate Studies in Mathematics. American Mathematical Society, Providence, RI, 2008.

[95] E. Jabotinsky. Analytic iteration. Trans. Amer. Math. Soc., $108: 457-477,1963$.

[96] E. Jabotinsky. $l$-sequences for nonembeddable functions. Proc. Amer. Math. Soc., 17 :738-741, 1966.

[97] A. G. Khovanskiur. Topological obstructions to the representability of functions by quadratures. J. Dynam. Control Systems, 1(1) :91-123, 1995.

[98] T. Kimura. On the iteration of analytic functions. Funkcial. Ekvac., $14: 197-238,1971$.

[99] M. Klughertz. Existence d'une intégrale première méromorphe pour des germes de feuilletages à feuilles fermées du plan complexe. Topology, 31(2) :255-269, 1992. 
[100] S. Kobayashi. Le groupe des transformations qui laissent invariant le parallélisme. In Colloque de topologie de Strasbourg, 1954-1955, page 5. Institut de Mathématique, Université de Strasbourg., 1954.

[101] G. Køenigs. Recherches sur les intégrales de certaines équations fonctionnelles. Ann. Sci. École Norm. Sup. (3ième Série), 1 :1-41, 1884.

[102] G. Kønigs. Nouvelles recherches sur les équations fonctionnelles. Ann. Sci. École Norm. Sup. (3ième Série), 2 :385-404, 1885.

[103] A. Korkine. Sur un problème d'interpolation. Bull. des sci. math. et astr. série 2, 6(2) :228-242, 1882.

[104] S. Lamy. Dynamique des groupes paraboliques d'automorphismes polynomiaux de $\mathbb{C}^{2}$. Bol. Soc. Brasil. Mat. (N.S.), 32(2) :185-212, 2001.

[105] H. B. Laufer. Normal two-dimensional singularities. Princeton University Press, Princeton, N.J.; University of Tokyo Press, Tokyo, 1971. Annals of Mathematics Studies, No. 71.

[106] L. Leau. étude sur les équations fonctionnelles à une ou plusieurs variables. Ann. Fac. Sci. Toulouse, $11: 1-110,1897$.

[107] P. Lévy. Fonctions à croissance régulière et itération d'ordre fractionnaire. Ann. Mat. Pura Appl. (4ième Série), 5 :269-298, 1928.

[108] M. Lewin. An example of a function with non-analytic iterates. $J$. Austral. Math. Soc., 5 :388-392, 1965.

[109] S. Lie. Sophus Lie's 1880 transformation group paper. Math Sci Press, Brookline, Mass., 1975. In part a translation of "Theorie der Transformations-gruppen" by S. Lie [Math. Ann. 16 (1880), 441528], Translated by Michael Ackerman, Comments by Robert Hermann, Lie Groups : History, Frontiers and Applications, Vol. I.

[110] A. Lins Neto. Construction of singular holomorphic vector fields and foliations in dimension two. J. Differential Geom., 26(1) :1-31, 1987.

[111] A. Lins Neto. Germs of complex two dimensional foliations. Bull. Braz. Math. Soc. (N.S.), 46(4) :645-680, 2015.

[112] A. Lins Neto and B. Scárdua. Complex algebraic foliations, volume 67 of De Gruyter Expositions in Mathematics. De Gruyter, Berlin, [2020] (C)2020.

[113] L. S. O. Liverpool. Fractional iteration near a fix point of multiplier 1. J. London Math. Soc. (2), 9 :599-609, 1974/75.

[114] L. López-Hernanz. Summable formal invariant curves of diffeomorphisms. Ergodic Theory Dynam. Systems, 32(1) :211-221, 2012.

[115] F. Loray. Feuilletages holomorphes à holonomie résoluble. Thèse de l'Université de Rennes 1, pages 1-126, 1994. 
[116] F. Loray. Rigidité topologique pour des singularités de feuilletages holomorphes. In J. Mozo Fernandez, editor, Ecuationes diferenciales y singularidades, pages 213-234. Universidad de Valladolid, 1995.

[117] F. Loray. Dynamique des groupes d'automorphismes de $\mathbb{C}, 0$. Bol. Soc. Mat. Mexicana (3), 5(1) :1-23, 1999.

[118] F. Loray. Réduction formelle des singularités cuspidales de champs de vecteurs analytiques. J. Differential Equations, 158(1) :152-173, 1999.

[119] F. Loray. Formal invariants for nonsolvable subgroups of $\operatorname{Diff}^{\omega}(\mathbb{C}, 0)$. J. Algebra, 247(1) :95-103, 2002.

[120] F. Loray. Towards the Galois groupoid of nonlinear O.D.E. In Differential equations and the Stokes phenomenon, pages 203-275. World Sci. Publishing, River Edge, NJ, 2002.

[121] F. Loray. Versal deformation of the analytic saddle-node. Astérisque, (297) :167-187, 2004. Analyse complexe, systèmes dynamiques, sommabilité des séries divergentes et théories galoisiennes. II.

[122] F. Loray. Sur les Théorèmes I et II de Painlevé. In Geometry and dynamics, volume 389 of Contemp. Math., pages 165-190. Amer. Math. Soc., Providence, RI, 2005.

[123] F. Loray and R. Meziani. Classification de certains feuilletages associés à un cusp. Bol. Soc. Brasil. Mat. (N.S.), 25(1) :93-106, 1994.

[124] F. Loray and J. C. Rebelo. Minimal, rigid foliations by curves on $\mathbb{C P}^{n}$. J. Eur. Math. Soc. (JEMS), 5(2) :147-201, 2003.

[125] F. Loray, M. van der Put, and F. Recher. Counting fixed points of a finitely generated subgroup of Aff $[\mathbb{C}]$. Publ. Mat., 48(1) :127-137, 2004.

[126] B. Malgrange. Travaux d'Écalle et de Martinet-Ramis sur les systèmes dynamiques. In Bourbaki Seminar, Vol. 1981/1982, volume 92 of Astérisque, pages 59-73. Soc. Math. France, Paris, 1982.

[127] B. Malgrange. Le groupoïde de Galois d'un feuilletage. In Essays on geometry and related topics, Vol. 1, 2, volume 38 of Monogr. Enseign. Math., pages 465-501. Enseignement Math., Geneva, 2001.

[128] B. Malgrange. On nonlinear differential Galois theory. Chinese Ann. Math. Ser. B, 23(2) :219-226, 2002. Dedicated to the memory of Jacques-Louis Lions.

[129] P. Mardešić, M. Resman, J.-P. Rolin, and V. Županović. The Fatou coordinate for parabolic Dulac germs. J. Differential Equations, 266(6) :3479-3513, 2019.

[130] D. Marín. Moduli spaces of germs of holomorphic foliations in the plane. Comment. Math. Helv., 78(3) :518-539, 2003. 
[131] D. Marín and J.-F. Mattei. Incompressibilité des feuilles de germes de feuilletages holomorphes singuliers. Ann. Sci. Éc. Norm. Supér. (4), 41(6):855-903, 2008.

[132] D. Marín and J.-F. Mattei. Monodromy and topological classification of germs of holomorphic foliations. Ann. Sci. Éc. Norm. Supér. (4), 45(3): :405-445, 2012.

[133] D. Marín and J.-F. Mattei. Topology of singular holomorphic foliations along a compact divisor. J. Singul., 9 :122-150, 2014.

[134] D. Marín, J.-F. Mattei, and É. Salem. Topological moduli space for germs of holomorphic foliations. Int. Math. Res. Not. IMRN, (23) :9228-9292, 2020.

[135] M. Martelo and J. Ribón. Derived length of solvable groups of local diffeomorphisms. Math. Ann., 358(3-4) :701-728, 2014.

[136] M. Martelo and B. Scárdua. On groups of formal diffeomorphisms of several complex variables. An. Acad. Brasil. Ciênc., 84(4) :873-880, 2012.

[137] M. Martelo and B. Scárdua. Integrating factors for groups of formal complex diffeomorphisms. J. Dyn. Control Syst., 19(2) :195-235, 2013.

[138] J. Martinet and J.-P. Ramis. Problèmes de modules pour des équations différentielles non linéaires du premier ordre. Inst. Hautes Études Sci. Publ. Math., (55) :63-164, 1982.

[139] J. Martinet and J.-P. Ramis. Classification analytique des équations différentielles non linéaires résonnantes du premier ordre. Ann. Sci. École Norm. Sup. (4), 16(4) :571-621 (1984), 1983.

[140] J.-F. Mattei and R. Moussu. Holonomie et intégrales premières. Ann. Sci. École Norm. Sup. (4), 13(4) :469-523, 1980.

[141] J.-F. Mattei, J. C. Rebelo, and H. Reis. Generic pseudogroups on $(\mathbb{C}, 0)$ and the topology of leaves. Compos. Math., 149(8) :1401-1430, 2013.

[142] J.-F. Mattei and É. Salem. Classification formelle de feuilletages singuliers de $\left(\mathbb{C}^{2}, 0\right)$. C. R. Acad. Sci. Paris Sér. I Math., 325(7) :773$778,1997$.

[143] J.-F. Mattei and É. Salem. Complete systems of topological and analytical invariants for a generic foliation of $\left(\mathbb{C}^{2}, 0\right)$. Math. Res. Lett., 4(1) :131-141, 1997.

[144] R. Meziani. Classification analytique d'équations différentielles $y d y+\cdots=0$ et espace de modules. Bol. Soc. Brasil. Mat. (N.S.), 27(1):23-53, 1996.

[145] R. Meziani and P. Sad. Singularités nilpotentes et intégrales premières. Publ. Mat., 51(1) :143-161, 2007. 
[146] J. Milnor. Dynamics in one complex variable. Friedr. Vieweg \& Sohn, Braunschweig, 1999. Introductory lectures.

[147] R. Moussu. Holonomie évanescente des équations différentielles dégénerées transverses. In Singularities and dynamical systems (Iráklion, 1983), volume 103 of North-Holland Math. Stud., pages 161-173. North-Holland, Amsterdam, 1985.

[148] R. Moussu. Sur l'existence d'intégrales premières holomorphes. Ann. Scuola Norm. Sup. Pisa Cl. Sci. (4), 26(4) :709-717, 1998.

[149] V. A. Naĭshul'. Topological invariants of germs of analytic mappings and area preserving mappings, and their application to analytic differential equations in $\mathbb{C} P^{2}$. Funktsional. Anal. i Prilozhen., 14(1) :73-74, 1980.

[150] V. A. Năshul'. Topological invariants of analytic and area-preserving mappings and their application to analytic differential equations in $\mathbb{C}^{2}$ and $\mathbb{C} P^{2}$. Trudy Moskov. Mat. Obshch., $44: 235-245,1982$.

[151] I. Nakai. Separatrices for nonsolvable dynamics on ( $\mathbb{C}, 0)$. Ann. Inst. Fourier (Grenoble), 44(2) :569-599, 1994.

[152] I. Nakai. The classification of curvilinear angles in the complex plane and the groups of \pm holomorphic diffeomorphisms. Ann. Fac. Sci. Toulouse Math. (6), 7(2) :313-334, 1998.

[153] I. Nakai and K. Yanai. Relations of formal diffeomorphisms. Kyoto University Research Information Repositary, 1447 :145-163, 2005.

[154] A. Newlander and L. Nirenberg. Complex analytic coordinates in almost complex manifolds. Ann. of Math. (2), 65 :391-404, 1957.

[155] L. Ortiz-Bobadilla, E. Rosales-González, and S. M. Voronin. Rigidity theorem for degenerated singular points of germs of holomorphic vector fields in the complex plane. J. Dynam. Control Systems, 7(4) :553-599, 2001.

[156] L. Ortiz-Bobadilla, E. Rosales-González, and S. M. Voronin. Rigidity theorems for generic holomorphic germs of dicritic foliations and vector fields in $\left(\mathbb{C}^{2}, 0\right)$. Mosc. Math. J., 5(1) :171-206, 2005.

[157] L. Ortiz-Bobadilla, E. Rosales-González, and S. M. Voronin. Analytic normal forms of germs of holomorphic dicritic foliations. Mosc. Math. J., 8(3) :521-545, 616, 2008.

[158] L. Ortiz-Bobadilla, E. Rosales-González, and S. M. Voronin. Extended holonomy and topological invariance of vanishing holonomy group. J. Dyn. Control Syst., 14(3) :299-358, 2008.

[159] D. Panazzolo. PSL $(2, \mathbb{C})$, the exponential and some new free groups. Q. J. Math., 69(1):75-117, 2018. 
[160] É. Paul. Formes abéliennes, formes résolubles. In J. Mozo Fernandez, editor, Ecuationes diferenciales y singularidades, pages 353-382. Universidad de Valladolid, 1995.

[161] É. Paul. Feuilletages holomorphes singuliers à holonomie résoluble. J. Reine Angew. Math., $514: 9-70,1999$.

[162] É. Paul. Formal normal forms for the perturbations of a quasihomogeneous Hamiltonian vector field. J. Dynam. Control Systems, 10(4) :545-575, 2004.

[163] É. Paul. On the Galois reducibility of a germ of quasi-homogeneous foliation. In Algebraic, analytic and geometric aspects of complex differential equations and their deformations. Painlevé hierarchies, RIMS Kôkyûroku Bessatsu, B2, pages 159-165. Res. Inst. Math. Sci. (RIMS), Kyoto, 2007.

[164] É. Paul. The Galoisian envelope of a germ of foliation : the quasihomogeneous case. Astérisque, (323) :269-290, 2009.

[165] J. V. Pereira and C. Perrone. Germs of integrable forms and varieties of minimal degree. Bull. Sci. Math., 134(1) :1-11, 2010.

[166] R. Pérez Marco. Solution complète au problème de Siegel de linéarisation d'une application holomorphe au voisinage d'un point fixe (d'après J.-C. Yoccoz). Astérisque, (206) :Exp. No. 753, 4, 273-310, 1992. Séminaire Bourbaki, Vol. 1991/92.

[167] R. Pérez Marco. Sur les dynamiques holomorphes non linéarisables et une conjecture de V. I. Arnol'd. Ann. Sci. École Norm. Sup. (4), 26(5): :565-644, 1993.

[168] R. Pérez Marco. Topology of julia sets and hedgehogs. Prépublications de l'Université de Paris-Sud, Orsay, 94-48 :1-45, 1994.

[169] R. Pérez Marco. Nonlinearizable holomorphic dynamics having an uncountable number of symmetries. Invent. Math., 119(1) :67-127, 1995.

[170] R. Pérez Marco. Fixed points and circle maps. Acta Math., 179(2) :243-294, 1997.

[171] R. Pérez Marco and J.-C. Yoccoz. Germes de feuilletages holomorphes à holonomie prescrite. Astérisque, (222) :7, 345-371, 1994. Complex analytic methods in dynamical systems (Rio de Janeiro, 1992).

[172] G. A. Pfeiffer. On the conformal mapping of curvilinear angles. the functional equation $\phi[f(x)]=a_{1} \phi(x)$. Trans. Amer. Math. Soc., $18: 185-198,1917$.

[173] S. Pinheiro and H. Reis. Topological aspects of completely integrable foliations. J. Lond. Math. Soc. (2), 89(2) :415-433, 2014. 
[174] Vincent R. and Fabien V.-T. Can we make sense out of "Tensor Field Theory"? arXiv :2101.04970 [math-ph], pages 1-34, 2021.

[175] J. Raissy. Linearization of holomorphic germs with quasi-Brjuno fixed points. Math. Z., 264(4) :881-900, 2010.

[176] J. Raissy. Holomorphic linearization of commuting germs of holomorphic maps. J. Geom. Anal., 23(4) :1993-2019, 2013.

[177] J. Raissy and L. Vivas. Dynamics of two-resonant biholomorphisms. Math. Res. Lett., 20(4) :757-771, 2013.

[178] J.-P. Ramis. Confluence et résurgence. J. Fac. Sci. Univ. Tokyo Sect. IA Math., 36(3) :703-716, 1989.

[179] J.-P. Ramis. Séries divergentes et théories asymptotiques. Bull. Soc. Math. France, 121(Panoramas et Syntheses, suppl.) :74, 1993.

[180] A. Ran. A non-embeddable composite of embeddable functions. $J$. Austral. Math. Soc., 8 :109-113, 1968.

[181] J. C. Rebelo and H. Reis. Cyclic stabilizers and infinitely many hyperbolic orbits for pseudogroups on $(\mathbb{C}, 0)$. J. Inst. Math. Jussieu, 13(2) :413-446, 2014.

[182] J. C. Rebelo and H. Reis. A note on integrability and finite orbits for subgroups of Diff $\left(\mathbb{C}^{n}, 0\right)$. Bull. Braz. Math. Soc. (N.S.), 46(3) :469490, 2015.

[183] J. C. Rebelo and H. Reis. Discrete orbits, recurrence and solvable subgroups of $\operatorname{Diff}\left(\mathbb{C}^{2}, 0\right)$. J. Geom. Anal., 27(1) :1-55, 2017.

[184] H. Reis. Equivalence and semi-completude of foliations. Nonlinear Anal., 64(8) :1654-1665, 2006.

[185] M. Resman. $\epsilon$-neighborhoods of orbits and formal classification of parabolic diffeomorphisms. Discrete Contin. Dyn. Syst., 33(8) :37673790, 2013.

[186] J. Rey. Difféomorphismes résonnants de $(\mathbb{C}, 0)$. Thèse de l'Université P.Sabatier, Toulouse, pages 1-121, 1996.

[187] J. Ribón. Difféomorphismes de $\left(\mathbb{C}^{2}, 0\right)$ tangents à l'identité qui préservent la fibration de Hopf. C. R. Acad. Sci. Paris Sér. I Math., 332(11):1011-1014, 2001.

[188] J. Ribón. Formal classification of unfoldings of parabolic diffeomorphisms. Ergodic Theory Dynam. Systems, 28(4) :1323-1365, 2008.

[189] J. Ribón. Modulus of analytic classification for unfoldings of resonant diffeomorphisms. Mosc. Math. J., 8(2) :319-395, 400, 2008.

[190] J. Ribón. Non-embeddability of general unipotent diffeomorphisms up to formal conjugacy. Ann. Inst. Fourier (Grenoble), 59(3) :951975, 2009. 
[191] J. Ribón. Unfoldings of tangent to the identity diffeomorphisms. Astérisque, (323) :325-370, 2009.

[192] J. Ribón. Topological classification of families of diffeomorphisms without small divisors. Mem. Amer. Math. Soc., 207(975) :x+166, 2010 .

[193] J. Ribón. Finite dimensional groups of local diffeomorphisms. Israel J. Math., 227(1) :289-329, 2018.

[194] J. Ribón. The solvable length of groups of local diffeomorphisms. $J$. Reine Angew. Math., 752 :105-139, 2019.

[195] J. Ribón. Topological rigidity of generic unfoldings of tangent to the identity diffeomorphisms. Ann. Inst. Fourier (Grenoble), 69(3) :9931046, 2019.

[196] C. Rousseau. The moduli space of germs of generic families of analytic diffeomorphisms unfolding of a codimension one resonant diffeomorphism or resonant saddle. J. Differential Equations, 248(7) :1794-1825, 2010.

[197] C. Rousseau. Analytic moduli for unfoldings of germs of generic analytic diffeomorphisms with a codimension $k$ parabolic point. Ergodic Theory Dynam. Systems, 35(1) :274-292, 2015.

[198] C. Rousseau and C. Christopher. Modulus of analytic classification for the generic unfolding of a codimension 1 resonant diffeomorphism or resonant saddle. Ann. Inst. Fourier (Grenoble), 57(1) :301-360, 2007.

[199] B. A. Scárdua. Transversely affine and transversely projective holomorphic foliations. Ann. Sci. École Norm. Sup. (4), 30(2) :169-204, 1997.

[200] E. Schröder. Ueber unendlich viele algorithmen zur auflösung der gleichungen. Math. Ann., 2 :317-365, 1870.

[201] A. Seidenberg. Reduction of singularities of the differential equation $A d y=B d x$. Amer. J. Math., $90: 248-269,1968$.

[202] B. Seke. Sur les structures transversalement affines des feuilletages de codimension un. Ann. Inst. Fourier (Grenoble), 30(1) :ix, 1-29, 1980 .

[203] A. A. Shcherbakov. Density of the orbit of a pseudogroup of conformal mappings and generalization of the Khudaĭ-Verenov theorem. Vestnik Moskov. Univ. Ser. I Mat. Mekh., (4) :10-15, 84, 1982.

[204] A. A. Shcherbakov. Topological classification of germs of conformal mappings with an identical linear part. Vestnik Moskov. Univ. Ser. I Mat. Mekh., (3) :52-57, 111, 1982. 
[205] A. A. Shcherbakov. Topological and analytic conjugation of noncommutative groups of germs of conformal mappings. Trudy Sem. Petrovsk., (10):170-196, 238-239, 1984.

[206] A. A. Shcherbakov. Complex limit cycles of the equation $d w / d z=$ $P_{n} / Q_{n}$. Uspekhi Mat. Nauk, 41(1(247)) :211-212, 1986.

[207] A. A. Shcherbakov, E. Rosales-González, and L. Ortiz-Bobadilla. Countable set of limit cycles for the equation $d w / d z=$ $P_{n}(z, w) / Q_{n}(z, w)$. J. Dynam. Control Systems, 4(4):539-581, 1998.

[208] C. L. Siegel. Iteration of analytic functions. Ann. of Math. (2), $43: 607-612,1942$.

[209] M. F. Singer. Liouvillian first integrals of differential equations. Trans. Amer. Math. Soc., 333(2) :673-688, 1992.

[210] L. Stolovitch. Classification analytique de champs de vecteurs 1résonnants de $\left(\mathbf{C}^{n}, 0\right)$. Asymptotic Anal., 12(2) :91-143, 1996.

[211] E. Stróżyna. The analytic and formal normal form for the nilpotent singularity. The case of generalized saddle-node. Bull. Sci. Math., 126(7) :555-579, 2002.

[212] E. Stróżyna and H. Żołądek. The analytic and formal normal form for the nilpotent singularity. J. Differential Equations, 179(2) :479$537,2002$.

[213] E. Stróżyna and H. Żołądek. Orbital formal normal forms for general Bogdanov-Takens singularity. J. Differential Equations, 193(1) :239259, 2003.

[214] H. J. Sussmann. Orbits of families of vector fields and integrability of distributions. Trans. Amer. Math. Soc., 180 :171-188, 1973.

[215] M. Suzuki. Sur les intégrales premières de certains feuilletages analytiques complexes. In Fonctions de plusieurs variables complexes, III (Sém. François Norguet, 1975-1977), volume 670 of Lecture Notes in Math., pages 53-79, 394. Springer, Berlin, 1978.

[216] G. Szekeres. Regular iteration of real and complex functions. Acta Math., 100 :203-258, 1958.

[217] G. Szekeres. Fractional iteration of entire and rational functions. $J$. Austral. Math. Soc., 4 :129-142, 1964.

[218] F. Touzet. Sur les intégrales premières dans la classe de Nilsson d'équations différentielles holomorphes. Trans. Amer. Math. Soc., 352(4) :1601-1622, 2000.

[219] F. Touzet. Rigidité de quelques groupes de germes de difféomorphismes. Publ. Mat., 47(2) :459-475, 2003.

[220] F. Touzet. Sur les feuilletages holomorphes transversalement projectifs. Ann. Inst. Fourier (Grenoble), 53(3) :815-846, 2003. 
[221] J.-M. Trépreau. Discrimination analytique des difféomorphismes résonnants de $(\mathbb{C}, 0)$ et réflexion de Schwarz. Astérisque, (284) :271319, 2003. Autour de l'analyse microlocale.

[222] H. Umemura. Differential Galois theory of infinite dimension. Nagoya Math. J., 144 :59-135, 1996.

[223] H. Umemura. Differential Galois theory of infinite dimension. Nagoya Math. J., 144 :59-135, 1996.

[224] M. van der Put and M. F. Singer. Galois theory of linear differential equations, volume 328 of Grundlehren der Mathematischen Wissenschaften [Fundamental Principles of Mathematical Sciences]. Springer-Verlag, Berlin, 2003.

[225] S. M. Voronin. Analytic classification of germs of conformal mappings $(\mathbb{C}, 0) \rightarrow(\mathbb{C}, 0)$. Funktsional. Anal. i Prilozhen., 15(1):1-17, 96, 1981.

[226] S. M. Voronin. Analytic classification of pairs of involutions and its applications. Funktsional. Anal. i Prilozhen., 16(2):21-29, 96, 1982.

[227] S. M. Voronin. Invariants for singular points of holomorphic vector fields on the complex plane. In The Stokes phenomenon and Hilbert's 16th problem (Groningen, 1995), pages 305-323. World Sci. Publ., River Edge, NJ, 1996.

[228] S. M. Voronin. Invariants for singular points of holomorphic vector fields on the complex plane. In The Stokes phenomenon and Hilbert's 16th problem (Groningen, 1995), pages 305-323. World Sci. Publ., River Edge, NJ, 1996.

[229] J.-C. Yoccoz. Linéarisation des germes de difféomorphismes holomorphes de (C, 0). C. R. Acad. Sci. Paris Sér. I Math., 306(1) :55$58,1988$.

[230] J.-C. Yoccoz. Théorème de Siegel, nombres de Bruno et polynômes quadratiques. Astérisque, (231):3-88, 1995. Petits diviseurs en dimension 1.

[231] S. Zakeri. Dynamics of singular holomorphic foliations on the complex projective plane. In Laminations and foliations in dynamics, geometry and topology (Stony Brook, NY, 1998), volume 269 of Contemp. Math., pages 179-233. Amer. Math. Soc., Providence, RI, 2001.

[232] H. Żołądek. The monodromy group, volume 67 of Instytut Matematyczny Polskiej Akademii Nauk. Monografie Matematyczne (New Series) [Mathematics Institute of the Polish Academy of Sciences. Mathematical Monographs (New Series)]. Birkhäuser Verlag, Basel, 2006. 


\section{Index}

$(\text { Eucl })_{\mathrm{col}},(\text { Eucl })_{\mathrm{col}}^{\mathrm{ex}}, 193$

(Fin) $)_{\mathrm{col}}, 188$

(Fin) $)_{\text {nœud }}, 188$

(Lin) $)_{\text {col }}, 188$

$(\text { Lin })_{\text {col }}^{\mathrm{ex}}, 193$

(Lin) $)_{\text {hyp }}, 188$

$D \cdot D, 214$

$J^{k}, 69$

$X_{p, \lambda}, 70$

$[f, g], 81$

$\operatorname{Diff}(U), 155$

$\mathbb{E}, \mathbb{A}, \mathbb{P}, 69$

$\mathbb{E}_{p, \lambda}, \mathbb{A}_{p}, 80$

$\operatorname{Diff}(\mathbb{C}, 0), 61$

$\widehat{\operatorname{Diff}}(\mathbb{C}, 0), 68$

$\exp (t \cdot X), \exp (X), 74$

$\mathcal{B}, 98$

$\mathcal{X}(\mathbb{C}, 0), 68$

$\operatorname{Orb}(z), 134$

$\phi_{X}^{t}, 73$

$\widehat{\operatorname{Cent}}(f), 77$

$\widehat{\mathcal{X}}(\mathbb{C}, 0), 72$

Éclatement, 202

Auto-intersection, 214

Centralisateur formel, 77

Chapelet de sphères, 115

Chapelet géométrique, 117

Chapelet polarisé orienté, 117

Col, 187

Cols, 189

Cols holomorphes, 188

Cols linéaires, 188

Commutateur (groupe), 81
Coordonnées de Leau-Fatou, 110

Courbe généralisée, 211

Courbe invariante, 183

Crochet de Lie, 68

Développante, 240

Développement asymptotique, 109

Dicritique, 210

Exponentielle d'un champ de vecteur, 74

Feuilles, 174

Feuilletage régulier, 174

Feuilletage singulier, 181

Flot holomorphe, 73

Formule de l'indice, 213

Générateur infinitésimal, 73

Groupe à 1 paramètre, 73

Hérisson, 139

Holonomie, 180

Holonomie projective, 216

Indice de Camacho-Sad, 212, 213

Jet d'ordre $k, 69$

Leau, 105

Nœud, 187

Noud-col, 187

Nœud-cols, 195

Noeuds linéaires, 188

Orbite, 134 
Pétales attractifs et répulsifs, 107

Petits diviseurs, 96

Plaques, 174

Pseudo-groupe, 155

Pseudo-groupe basique, 176

Pseudo-groupe tangent, 176

Pseudo-groupe transverse, 177

Pseudo-groupe trivial, 179

Pseudo-groupes équivalents, 178

Résoluble (virtuellement), métabélien, 80

Résonant (difféomorphisme), 77

Séparatrice, 183

Secteurs et séparatrices de Nakai, 145

Singularité dégénérée, 186

Singularité de Poincaré-Dulac, 187

Singularité hyperbolique, 187

Singularité nilpotente, 186

Singularité réduite, 187

Singularité radiale, 187

Singularités (d'un feuilletage), 182

Singularités hyperboliques, 188

Système de coordonnées euclidiennes,

112

Théorème de la Fleur, 105

Topologie de Krull, 72

Transversale complète, 179

Variété centrale, 196

Variété forte, 196

Frank Loray

Univ Rennes,

CNRS, IRMAR - UMR 6625,

F-35000 Rennes, France

frank.loray@univ-rennes1.fr

https://perso.univ-rennes1.fr/frank.loray/ 